Doctorado en Ingeniería Ambiental, Química y de los Materiales Escuela Técnica Superior de Ingenieros Industriales Universidad Politécnica de Madrid

\title{
Label-free Optical Biosensors based on Resonant Nanopillars
}

Author: Ana López Hernández. Licenciada en Ciencias Ambientales

Directors: Dr. Miguel Holgado Bolaños. Ingeniero Industrial

Dra. María Fe Laguna Heras. Licenciada en Ciencias Químicas 


\section{Court Members}

President

D. Gustavo Adolfo Narros Sierra

Secretary

D. Alvaro Lavín Hueros

\section{Vocals}

D. Antonio Quesada del Corral

D. Sidharam Pujari

Dña. María José Bañuls Polo

Substitutes

Dña. María Jesús Moreno Bondi

D. Daniel Granados Ruiz 


\section{Agradecimientos}

Una tesis doctoral es el resumen del trabajo realizado durante unos años. En esos años uno no está solo, por ello las tesis no son solo de quien las escribe, si no de todas las personas e instituciones que rodean y apoyan al autor durante sus años de trabajo.

Esta tesis se ha desarrollado en el marco de un Proyecto europeo denominado Enviguard, que ha posibilitado la financiación de todo mi trabajo, durante el tiempo que he estado trabajando en la UPM, por ello debo agradecer tanto a la Universidad Politécnica de Madrid como a la Comisión Europea el apoyo económico prestado.

Además me gustaría dar las gracias al Consejo Social de la UPM ya que su ayuda económica, ha hecho posible la realización de una estancia en el extranjero durante tres meses, ampliando por ello los horizontes del trabajo de investigación que se presenta. En este contexto agradezco a la Universidad de Wageningen que me acogió con cariño e ilusión desde el primer momento.

Al Centro Láser de la UPM, por ser el Centro que me dio la bienvenida al fabuloso mundo de la investigación y por brindarme tan buenos momentos.

Agradezco al Centro de Tecnología Biomédica todo el esfuerzo llevado a cabo para que los proyectos y los grupos de investigación que alberga sigan creciendo día a día. Verdaderamente es un placer venir todos los días a trabajar a un Centro donde las personas se apoyan y las iniciativas se materializan.

Mis directores de tesis Miguel y Marifé han hecho posible que yo pueda llegar hasta aquí. Desde aquel día que entré en su despacho de la ETSII allá en septiembre de 2012, sin imaginarme que se convertirían en personas tan cercanas a mi, me han apoyado, comprendido, enseñado, dedicado horas y horas, me han metido caña y me han hecho reír y aprender como nunca. Definitivamente les voy a echar muchísimo de menos en esta nueva etapa que se avecina. Por todo ello, ¡Gracias!

Además compartir despacho con mis compañeros del Grupo de Óptica, Fotónica y Biofotónica estos años, ha sido no solo apasionante desde el punto de vista científico, sino también muy divertido y estimulante a nivel personal. Desde que empecé en el grupo como estudiante de fin de master el grupo ha crecido y cada día es más multidisciplinar, lo que nos hace crecer y aprender juntos. A mis compañeros les agradezco enormemente que hayan estado ahí siempre que lo he necesitado.

Aunque ahora somos muchos, y quiero a todos por igual, me gustaría agradecer especialmente a Rafa todo el tiempo que ha empleado explicándome cosas de óptica y de la vida, ha sido un pilar fundamental en el desarrollo de esta tesis. Tambien quiero dar las gracias Javi, porque con él empezaron mis primeros días en "labo" y la por paciencia que tuvo conmigo cuando entré en el grupo. Y cómo no a 
mi querida Betxu, gran profesional y gran persona que me ha ayudado en todo y que me contagiaba de energía cada mañana. ¡Eskerrik asko!

También merece una mención especial todo el equipo BIOD, siempre dispuestos a ayudarme en lo que sea con una sonrisa en la cara. Vivir en el ecosistema BIODUPM ha sido muy fructífero y me ha proporcionado mucho apoyo técnico y también muchas risas dentro y fuera del laboratorio.

Me gustaría dedicar esta tesis a mis padres, que han estado muy presentes durante todos estos años, apoyándome e interesándose por mis avances científicos. Es gracias a ellos por lo que he podido ser libre y escoger el camino que más me apetecía. También a mi sobrina, que llegó a este mundo, un día de esos "malos" en el laboratorio en los que nada sale y todo se estropea, para ilusionarme e iluminarme cada vez que pensaba en su divertida sonrisa.

Quiero compartir este trabajo con mi familia y amigos. Alguno estará pensando que con cuales exactamente, y es que por suerte, tengo muchos. A TODOS ¡Gracias!

Javi, mi pareja, se podría decir que definitivamente es parte de esta tesis, ya que no podría haberlo hecho sin él, o por lo menos, me hubiera costado mucho más, especialmente esta última etapa de escritura, (él lo sabe), nadie me ha comprendido ni robado tantas y tantas carajadas como él. De todo corazón.

Por último, no podría obviar en los agradecimientos, a mis cuatro preciosas hermanas que me dan tantos buenos y malos momentos, todos ellos parte de una vida que es un placer compartir con ellas. 
We should all think that knowledge comes from our senses, so if we extent our senses we will consequently extend our knowledge.

Neil Harbisson. 2012 Avant-garde artist and first cyborg activist 


\section{Resúmen}

El trabajo de investigación desarrollado en esta tesis está centrado en la obtención de un biosensor óptico, que no necesite amplificación química (sin marcado), que tenga alta sensibilidad y un coste bajo. Este biosensor está basado en redes de nanopilares resonantes (R-NPs) compuestos por pares de Reflectores de Bragg de nitruro de silicio y óxido de silicio $\left(\mathrm{Si}_{3} \mathrm{~N}_{4} / \mathrm{SiO}_{2}\right)$ y una cavidad central de óxido de silicio $\left(\mathrm{SiO}_{2}\right)$, dispuestas sobre un sustrato de cuarzo. Un nanopilar resonante tiene una respuesta óptica que consiste en una banda espectral que impide la transmisión de la luz (gap fotónico) salvo en un rango concreto de dicha banda, donde la luz se transmite (resonancia), debido a la cavidad central. La luz es guidada por cada R-NP que, debido a su carácter nanométrico, hace que parte de la luz viaje por fuera del nano-pilar observando lo que hay en su superficie, lo que se conoce como detección por campo evanescente. Aunque cada R-NP actúa como un único nanosensor, sería muy complicado observar la respuesta de un solo R-NP debido a su reducido tamaño, por ello se realizan redes o matrices de estos nanosensores, cuya proximidad produce un efecto de concentración de luz fuera de la superficie de los R-NPs, a este fenómeno se le conoce como concentración de la luz por campo evanescente. Además, estas celdas permiten que una muestra biológica líquida se infiltre en la matriz formada por los nasnosensores, y por ende se puedan observar: por un lado el cambio de las propiedades ópticas del líquido, y por otro, la inmobilización de un bioreceptor y las reacciones de afinidad entre el bioreceptor mencionado y la molécula objetivo a detectar (analito).

Uno de los desarrollos llevados a cabo en esta tesis es la fabricación de los R-NPs, siendo agrupados en celdas dispuestas en distintas zonas de un mismo chip, que, una vez tapizadas con el bioreceptor, se llamarán BICELLs (de su traducción al inglés Bio Photonic Sensing Cells). Esta distribución, dota a los R-NPs la posibilidad de ser utilizados como sensores con alta capacidad de multiplexación o capacidad para poder detectar muchas biomoléculas diferentes en un mismo chip.

Además de su diseño, fabricación y caracterización, en este trabajo se analiza el rendimiento de los R-NPs para ser utilizados como sensores químicos, ya que, 
mediante un conjunto de experimentos de sensado de las propiedades ópticas del líquido inflitrado (bulk sensing) se puede observar que las redes de nano-sensores tienen capacidad para diferenciar pequeños cambios en el índice de refracción del medio que les rodea, siendo así ideales para poder distinguir diferentes components del líquido y correlacionarlo con propiedades como la densidad, la viscosidad, la turbidez, entre otras.

También se ha probado la capacidad de los R-NPs para detectar la inmovilización de los biorreceptores y el posterior reconocimiento específico del analito. Para ello, se trabaja en la modificación de la superficie de los R-NPs y en distintos protocolos de biofuncionalización. En este sentido se observa que efectivamente, son capaces de inmovilizar biomoléculas y detectar analitos, ya sea en ambiente seco, o inmersos en el medio de transporte. Sin embargo, se concluye que la sensibilidad de los R-NPs aumenta más de diez veces cuando éstos son interrogados en medio seco. Este efecto es principalmente debido a que el contraste de índice de refracción que existe entre el aire (medio seco) y los R-NPs es mucho mayor al contraste de índice de refracción que existe entre el agua (medio húmedo) y los R-NPs.

Otra conclusión extraída del trabajo es que el pre-tratamiento de la de la superficie de los R-NPs es clave para alcanzar una biofuncionalización óptima en la que se maximice la detección del analito en cuestión. 


\section{SUMMARY}

The research work developed in this thesis is focused on obtaining an optical biosensor, which does not need chemical amplification (label-free), which has high sensitivity and is cost efficiency. This biosensor is based on arrays of resonant nanopillars (R-NPs), composed of pairs of Bragg reflectors of silicon nitride and silicon oxide $\left(\mathrm{Si}_{3} \mathrm{~N}_{4} / \mathrm{SiO}_{2}\right)$ and a central cavity of silicon oxide $\left(\mathrm{SiO}_{2}\right)$ arranged on a quartz substrate. A resonant nanopillar has an optical response consisting of a spectral band gap that prevents the light transmission (photonic gap) except in a specific range of the band, where the light is transmitted (resonance), due to the central cavity. The light is guided by each R-NP, and due to their nanometric character, part of the light travels outside of the nanopillar, observing thus, what is on the surface. This is known as evanescent field detection. Although each R-NP acts as a single nanosensor, it would be very difficult to observe the response of a single R-NP because of its small size, so R-NPs are arranged into arrays. Their proximity produces an effect of light concentration outside of the surface of the R-NPs. This phenomenon is known as concentration of light by evanescent field. In addition, this arrays, allow biological sample to infiltrate the matrix formed by the R-NPs, and thus they can observe: on one hand the change in the optical properties of the liquid, and on the other hand, the immobilization of a bioreceptor, and the affinity reactions between such bioreceptor and the target molecule to be detected (analyte).

One of the developments accomplished in this thesis is the fabrication of the R-NPs, being grouped in cells or arrays arranged in different zones of the same chip that, once immobilized with the bioreceptor, will be called BICELLs (Bio Photonic Sensing Cells). This distribution, gives R-NPs the possibility of being used as sensors with high multiplexing capacity, to be able to detect different biomolecules in the same chip.

Besides to the design, fabrication and characterization, this work, analyzes the performance of R-NPs to be used as chemical sensors, since, through a set of sensing experiments of the optical properties of the infiltrated liquid (bulk 
sensing), it can be observed that the R-NPs have the capacity to differentiate small changes in the refractive index (RI) of the media surrounding them. Being just ideal to be able to distinguish different components of the liquid and correlate them with properties such as density, viscosity, and turbidity, among others.

Also, it has been shown, the ability of the R-NPs to detect the immobilization of the bioreceptors and the subsequent specific recognition of the analyte. To do this, work was addressed to the surface modification of the R-NPs as well as to different biofunctionalization protocols. In this sense, it has been observed that R-NPs are effectively able to immobilize biomolecules and detect analyte, either in dry conditions or immersed in the transport medium. However, it is concluded that the sensitivity of the R-NPs increases more than ten times when they are interrogated in dry conditions. This effect is mainly due to the fact that the refractive index contrast between air (dry conditions) and R-NPs is much higher than, that, between the humid medium and R-NPs.

Another conclusion drawn from the work is that the pre-treatment of the surface of the R-NPs is a key factor to achieve an optimal biofunctionalization in which the detection of the analyte is maximized. 


\section{TABLE OF CONTENTS}

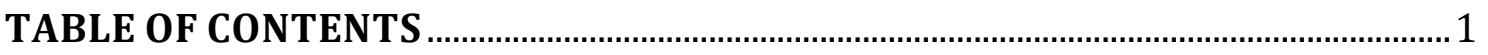

1. GENERAL INTRODUCTION ............................................................................................

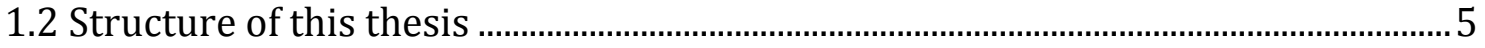

2. INTRODUCTION TO BIOSENSORS.....................................................................................

2.1 Operating principles of a biosensor................................................................................

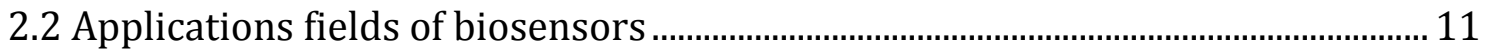

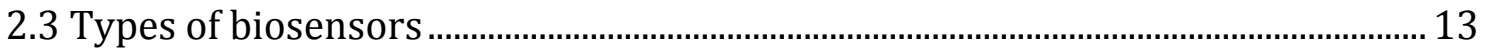

2.3.1 Classification of biosensors according to the transducing signal ........................13

2.3.2 Bioaffinity Biosensors: Immunoassays …………………………………………..... 16

2.4 Optical Biosensors................................................................................................... 23

2.4.1 Optical Labeled Biosensors …………………………………………………………... 24

2.4.2 Optical Label-free Biosensors.................................................................................... 26

2.4.3 Biofunctionalization techniques............................................................................... 35

2.5 Figures of merit of a biosensor ………………………………………………………... 43

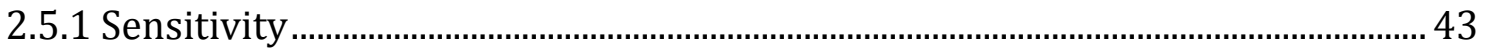

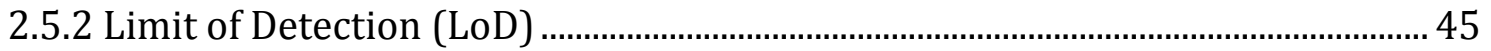

2.5.3 FWHM and Q-factor ........................................................................................... 46

3. OBJECTIVES OF THIS THESYS AND MOTIVATION OF THE USE OF R-NPS..... 47

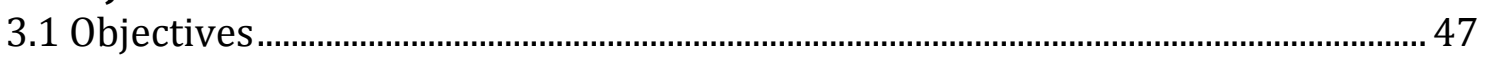

3.2 Why R-NPs as biological sensors? .................................................................................. 48

4. RESONANT NANOPILLARS ARRAYS …………………………………………… 51

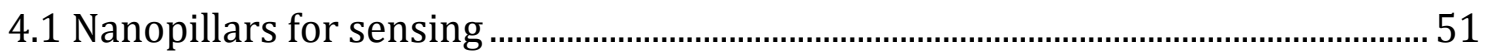

4.2 Photonic properties of the Resonant Nanopillars ...................................................... 54

4.2.1 Distributed Bragg Reflectors (DBR) for R-NPs ........................................................ 58

4.2.2 Calculation of the effective RI for R-NPs design ......................................................61

4.2.3 Influence of the Bragg Reflectors number in R-NPs ................................................63

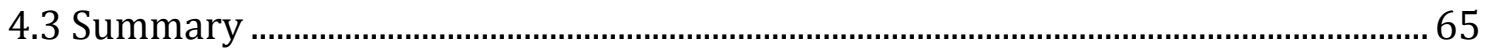

5. FABRICATION OF R-NPs.........................................................................................6 67

5.1 General introduction to micro-nano fabrication techniques.....................................67

5.1.1 Thin film layer deposition ............................................................................................67

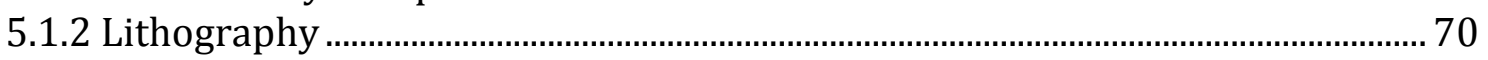

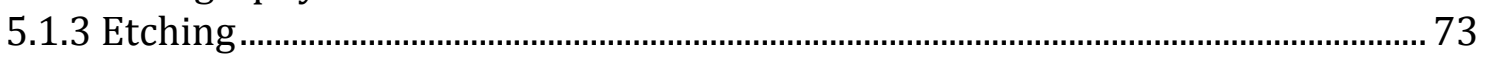

5.2 Fabrication process of R-NPs .................................................................................... 75

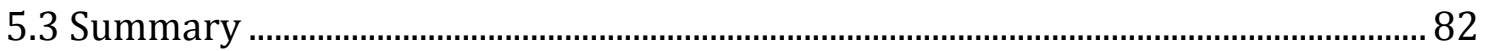


6. OPTICAL INTERROGATION OF R-NPS

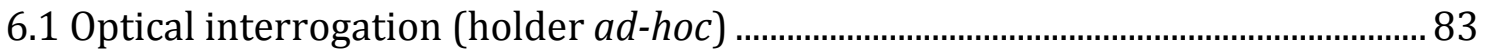

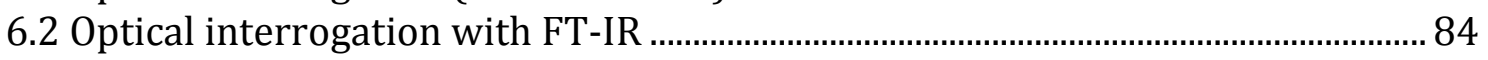

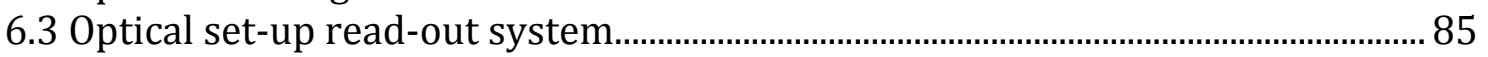

6.4 Fluidic system coupled to the optical set-up read-out system ...................................... 88

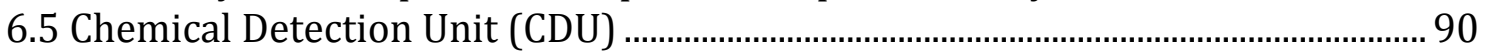

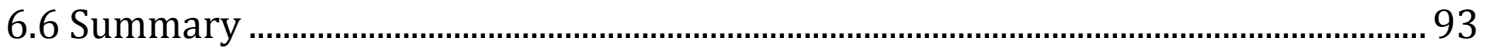

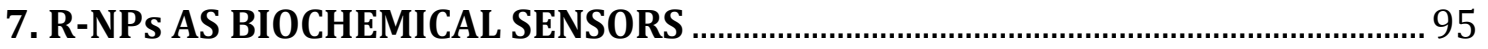

7.1. Bulk sensing performance of $\mathrm{SiO}_{2}$-NPs versus R-NPs................................................ 95

7.2 Influence of the number of the Bragg Reflectors ........................................................98

7.3 Effect of the lattice parameter on R-NPs with 10 pairs of Bragg Reflectors .....100

7.4 Static Bulk sensing experiment on R-NPs as biochemical sensors .......................101

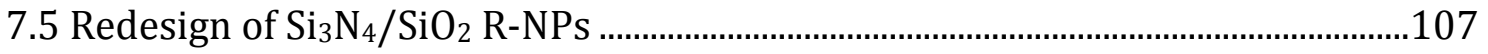

7.6 Multiplexing capacity of R-NPs and new lattice parameter ....................................109

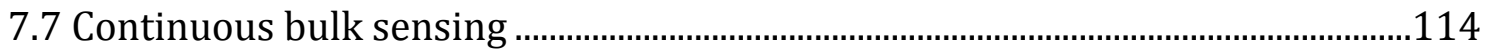

7.7.1 Real-Time response as a function of the absorbance...........................................115

7.7.2 Real-time response measured in reflectance .......................................................118

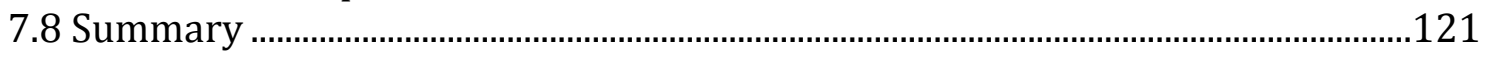

8. R-NPS ARRAYS FOR LABEL-FREE BIOSENSORS ……...........................................123

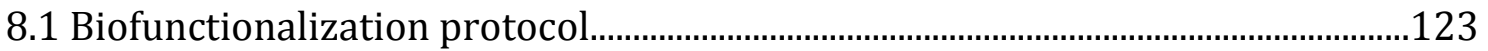

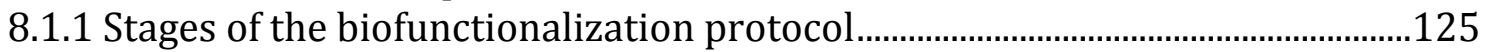

8.1.2 Biofunctionalization strategies carried out within this work ...........................129

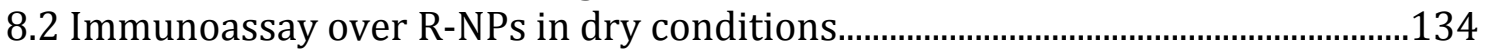

8.3 Immunoassay over R-NPs immersed in water ...........................................................138

8.4. Immobilization of R-NPs in continuous flow ..........................................................140

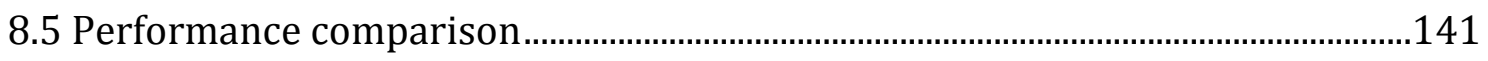

8.5.1 Immobilization of R-NPs: procedures comparison ..............................................141

8.5.2 Biosensing performance of R-NPs: under dry conditions or

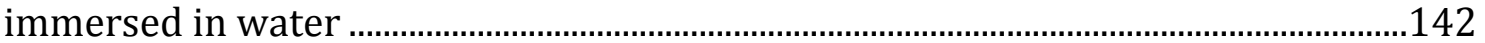

8.6 Surface modification techniques developed in Wageningen..................................151

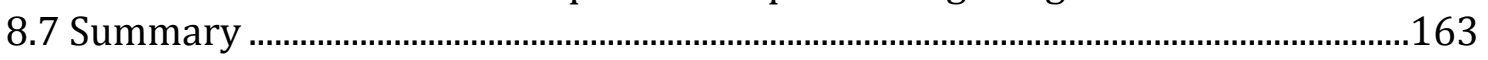

9. GENERAL CONCLUSIONS AND FUTURE PROSPECTS .............................................165

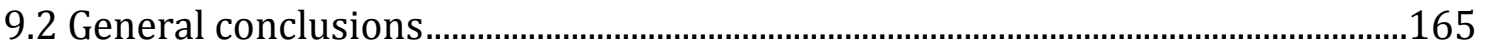

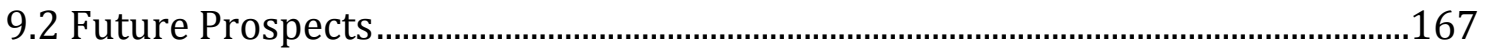

9.2.1 New lines of R-NPs biofunctionalization ...............................................................167

9.2.2 New materials for R-NPs development ...................................................................169

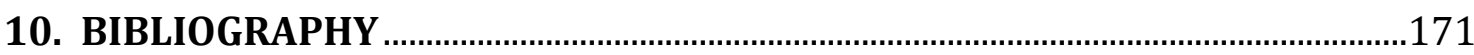

10.1 Publications of the author .......................................................................................171

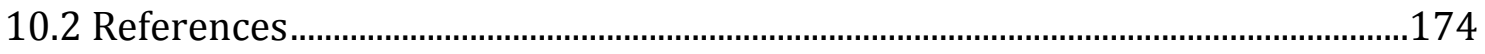




\section{GENERAL INTRODUCTION}

An intrinsic characteristic of humans is the need to be aware of the world that surrounds them. For this, the five senses: sight, touch, smell, touch and hearing have traditionally been used. However, all these senses have spatial and temporal limitation against certain physical-chemical events. There are imperceptible magnitudes for the human being.

For this reason, different systems of detection were devised several centuries ago covering the need to have more and better analytical information of the surrounding in general. First, the industrialization process in developed countries normalized the use of basic sensors, such as mercury-based thermometer or a simple photographic camera. Since then, sensors became a basic device for the system operationally. But it was during XX century, when they really aroused as a key player of the industrial and technology development, especially due to the two Words Wars and the Space Race. Pressure, electrochemical, magnetic, humidity, speed, distance, and so on, are some examples of these sensors. So much they became popular, that, currently in occident, they are used for multiple purposes in an intensive way in daily tasks. In fact they are present in the household appliances, transport, mass media...

So many are the applications, that they are only limited by the imagination itself.

One of these practices is the environmental surveillance or control. [Denninson, 1995]. Small changes in the environmental elements, can suggest that a severe or dramatic events could occur, affecting both, the health of the ecosystem or the natural resource itself, and human health direct or indirectly. For this, there exist extensive quantities of sensors that measure physical-chemical parameters. Nevertheless for the detection of biological parameters, sensors include a biological receptor; this bioreceptor turns a sensor into a biosensor. The biosensor is a device used to detect the presence and the concentration of a particular biomolecule within a target sample [Turner, 1987], [Cooper, 2009]. 
One of the technical obstacles to use the biosensors for environmental monitoring is the broad number and wide range of pollutants present in the different natural resources. Specially, in marine environment there can be found thousand and thousands of different bio-hazard compounds which should be noticed as soon as possible. Nevertheless, there should be a starting point, and this inevitably will be marked by the potential damage of a marine pollutant in human health. Thus, high sensitivity biosensors, such as the resonant nanopillars arrays proposed in this thesis, are definitely needed for the multianalyte detection.

In fact The European Commission has made water protection one of its priorities implementing new policies such as Marine Strategy Framework Directive (MSFD), the Directive 2010/75/EU on industrial emissions (integrated pollution prevention and control) and the Water Framework Directive 2000/60/EC. Therefore in the Seventh Framework Program they included the theme "Ocean" as a response to the growing need for accurate real-time monitoring of the seas/oceans and the aquaculture industries need for a reliable and cost-effective risk management tool.

From this need, Projects like Braavoo [Bravoo.org], Sea-on-a-chip [sea-on-achip.eu] or Enviguard [enviguard.net] were born.

The group of Optics, Photonics and Biophotonics (GOPB) from the Universidad Politécnica de Madrid (UPM) is one of the partners of Enviguard Project, which is focused on the development of a highly specific and precise in situ measurement device for currently, hard to measure man-made chemical contaminants and biohazards (toxic microalgae, bacteria, and chemical pollutants), that can be used as an early warning in aquaculture and as an environmental monitor to assess the status of the sea.

Since algae and bacteria biosensors are more commonly used, probably, the big challenge of the Project was the development of a high performance, low cost and reusable biosensor, for chemical pollutant detection. For that reason UPM developed the Resonant Nanopillars based optical transducers, which are the object of study in this thesis. Thus, this research work is framed in the Enviguard 
Project, which has established the milestones and the work rate during these last three years.

Despite Enviguard is the starting point of the research on the R-NPs performance as biological sensors, their use is not limited to water pollutant detection, but they can be applied for different species detection in many other fields.

\subsection{Structure of this thesis}

This thesis is divided in nine different chapters.

Chapter 1. General Introduction: Is a general introduction to the topic of the thesis and a description of the structure of the thesis.

Chapter 2. Biosensors, state of the art: Describes the state of the art of the biosensors. The basic operationally of a biosensor, the different classifications of the biosensors, the biofunctionalization techniques and other aspects influent on the technology of biosensors.

Chapter 3. Objectives of this thesis and motivation of the use of R-NPs: I lists a number of aims to be accomplished. Also there are explained the reasons of the use of R-NPs as label-free optical biosensors.

Chapter 4. Resonant Nanopillars Arrays: In this chapter there are reported the fundamental reasons that support the scientific study developed within this thesis It is explained the optical and photonics characteristics of the R-NPs, that leads to their use as label-free optical biosensors.

Chapter 5. Fabrication of the R-NPs: It describes the different technologies and process carried out for the R-NPs fabrication.

Chapter 6. Optical Interrogation of the R-NPs: There are reported the optical interrogation methodologies used for the R-NPs measurements. 
Chapter 7. R-NPs as biochemical sensors: This chapter shows the bulk sensing experiments developed for the demonstration of the adequacy to use R-NPs for biochemical detection.

Chapter 8. R-NPs as label-free optical detection: This chapter reports the experiments accomplished for the biological detection with the R-NPs.

Chapter 9. General conclusions and future prospects: It summarizes the conclusions drawn from this scientific work and highlights a series of works and experiments to be accomplished in the line of the obtained results.

Chapter 10. Bibliography: Reports the scientific publications of the author and the references used for the development off this document. 


\section{INTRODUCTION TO BIOSENSORS}

A biosensor is a device used for the measurement of biochemical or biological parameters. It combines a biological component (biorreceptor) and a physicochemical element (transducer) in which biological events such affinity and specificity reactions produce a change in the physical properties that can be translated into a quantifiable signal for its interpretation by the final user. Biosensors hold enormous potential: they can monitor personal health and fitness, the food quality, and our environment status. They can replace the large analytical facilities of industrial and health services with cheap and simple devices anyone can use. [Turner, 1987].

Celebrating the $20^{\text {th }}$ anniversary of the journal Biosensor and Bioelectronics, in the year 2005, Doctor Anthony P.F. Turner, who is probably one of the most relevant researchers in the field of biosensors worldwide, published a paper about the evolution of biosensors from the middle $80 \mathrm{~s}$, when the biosensors market really started. By that time that market was quantified in 5 million dollars, but by the year 2005, it had reached the 5 billion of dollars. [Turner, 2005]. This author claims that the absolute success of this sector relies on its multidisciplinary dimension, which involves chemistry, physics, biology, and engineering skills for the technology development. It combines imagination and practical objectives to satisfy the wish of a longer and better a quality of life.

To understand the success of this technology, both at a research and at a commercial level it is essential to know the principles of operation of the biosensors and also their final applications.

\subsection{Operating principles of a biosensor}

According to the International Union of Pure and Applied Chemistry (IUPAC), a biosensor is a device capable of providing specific quantitative or semi- 
quantitative analytical information using a biological recognition element (bioreceptor), which is retained in direct spatial contact with a transducing element. [Thevenot, 2001], [IUPAC]. (Figure 1).

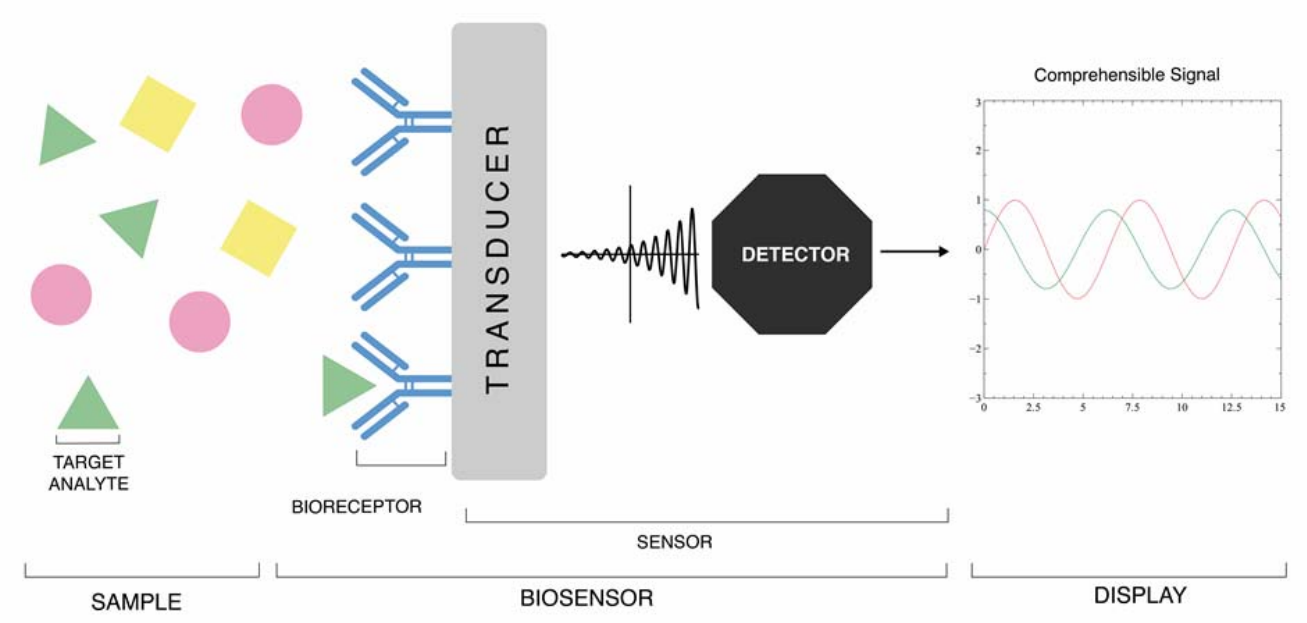

Figure 1: Scheme of the different components of a biosensor.

The bioreceptor must be attached or immobilized on the transducer. The sample to analyze is placed in contact with this bioreceptor, which will react with the target analyte present in the sample. The transducer emits a signal according to the biological event that is taking place, which is identified by the detector, and transformed into comprehensible signal. Biosensors may be classified according to the biological specificity conferring mechanism (bioreceptor) or, alternatively, to the mode of physico-chemical signal transduction [Thevenot, 2001]. In figure 1, the bioreceptor is represented as an antibody, appealing to the specific recognition of antigen-antibody in bio-affinity biosensors [Shankaran, 2007]. But there exist many different molecular recognizing materials: enzymes, virus, proteins and DNA chains. The transducer can be optical, mechanical, chemical, etc. Its goal is to "translate" the biological events into a quantifiable signal. (Figure 2). The transducing signal, normally needs to be processed with specific software's, that fortunately are increasing in capacity and rapidity, making biosensor suitable for 
rapid test analyses and in-situ diagnosis and Point of Care (PoC) devices easy to use.

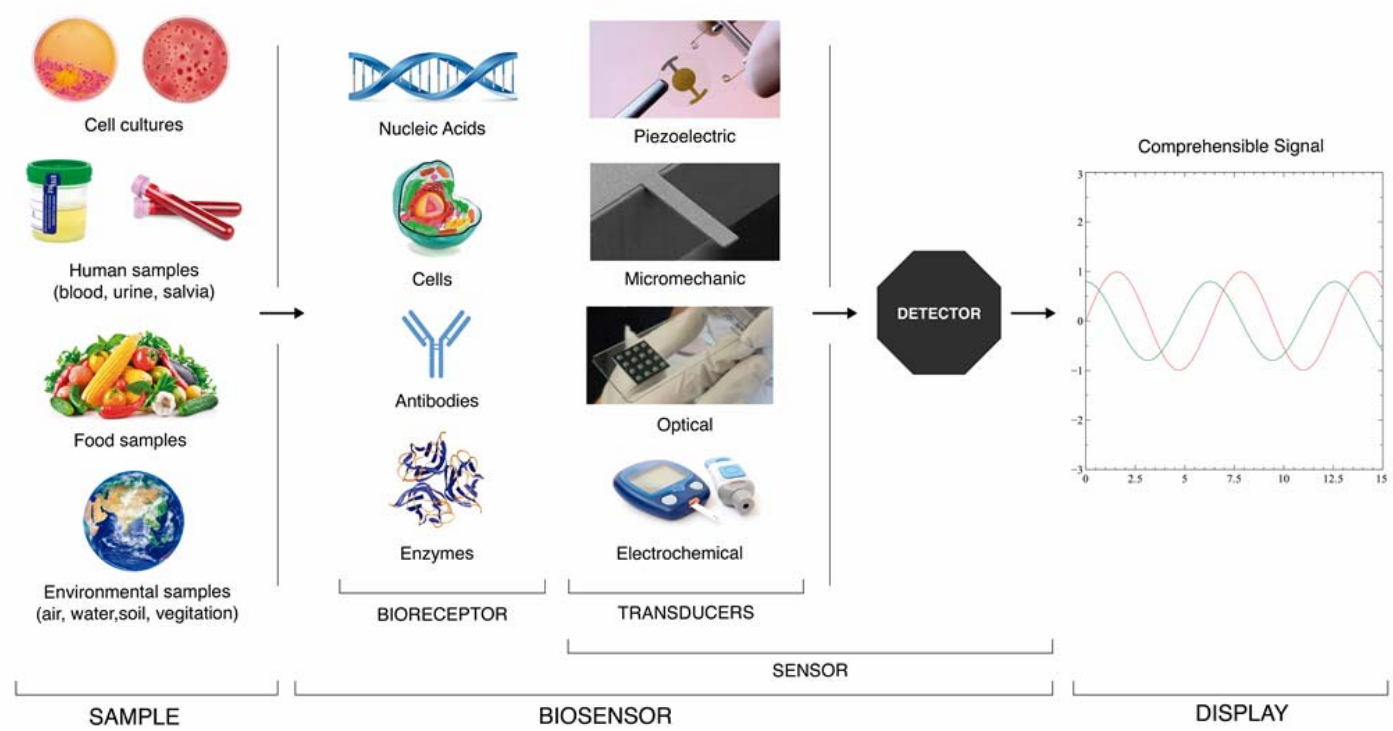

Figure 2: Elements that are part of a biosensor according to different requirements.

The first operative biosensor was the glucose transducer introduced by Clarck and Lyons in 1962. The researchers and the industry continued working in this line, to produce a low cost glucose biosensor, less time-consuming and easier to use. Then in 1975 Yellow Spring Ins. Company commercialized the first glucose biosensor. Since then, people with diabetes have experienced an unprecedented increase in the quality of life.

Glucose biosensor is a biocatalitic sensor capable of detecting the glucose concentration in blood samples in a matter of seconds. The bioreceptor used is the enzyme glucose oxidase immobilized over an oxygen electrode. This electrode detects the oxygen decreased caused by the activity of the enzyme. This decrease is directly proportional to the glucose concentration in blood. Biocatalitic sensors are normally used for vitamins, sugar or toxic species detection.

Since Clark and Lyons introduced the first biosensor in 1962, there has been an increasing demand for such analytical devices in diagnostic applications. Researchers initially focused mainly on detector principles and recognition 
elements, whereas the packaging of the biosensors and the microfluidic integration has been discussed only more recently. [Song, 2006]. The XXI century is the century of the nanotechnology and the promptness. Now one of the biggest challenges on the biosensors industry and research field is the development of Point of Care (PoC) systems. They are well-performing analytical devices (involving sample handle, bioreceptors, trasnducers, detectors and data processing) which must fulfill several technical characteristics, to become user-friendly, cost-effective, simple and portable, for real-time in-situ diagnoses. [Estevez, 2012]. There can be seen some examples in next figure.
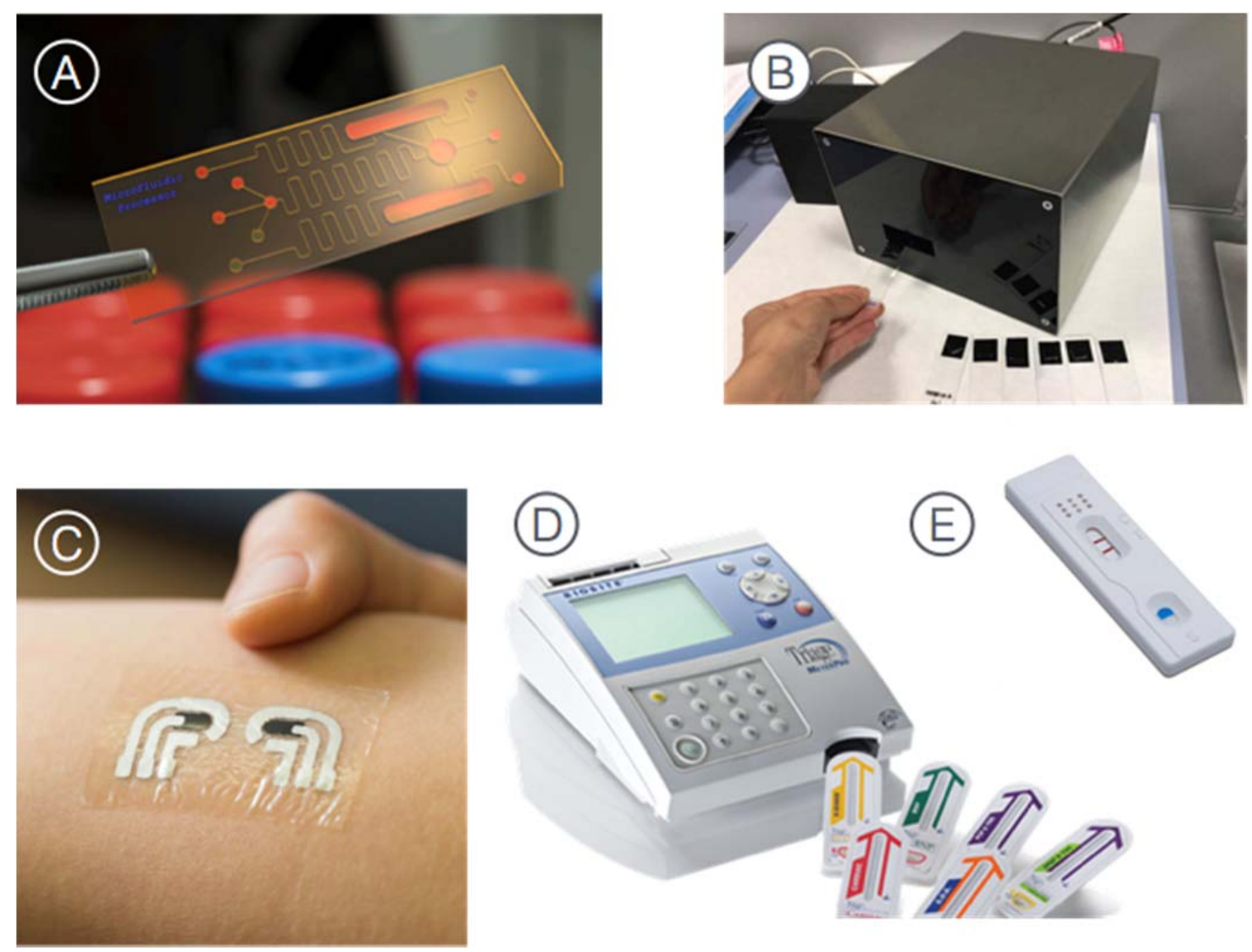

Figure 3: Examples of Point of Care devides. A. Lab-on-a-chip sensor. B. PoC for eye deseases detection. C. Skin steaker for glucose measurement. D. Rapid test for lateral flow sensors. $E$. Typical lateral flow pregnancy test. [phys.org]. 


\subsection{Applications fields of biosensors}

There exists a wide range of biosensors applications. (Figure 4). Some of these applications are food processing and safety control, environmental monitoring in different media, like atmosphere, water bodies, plants, animals, and many other natural resources, also biosensors are used in clinical analyses, defense, industries or medical field [Mehrotra, 2016].

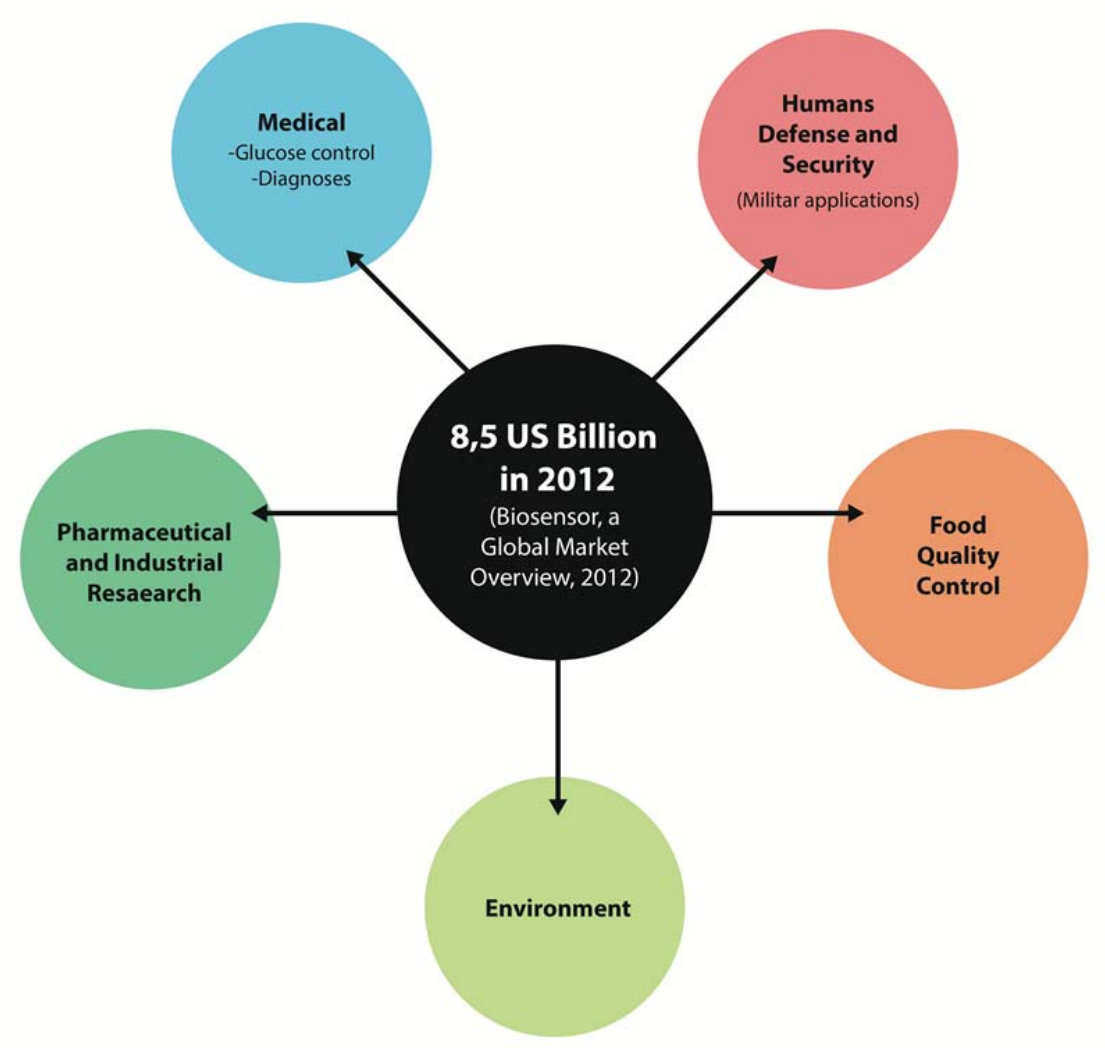

Figure 4: Applications of biosensors.

The clinical biosensors application is probably the most widespread regarding other applications. The Global Market Insights published in August 2016 a report studying the biosensors market size by application and estimated that the prevalence of the clinical application of the biosensors will continue at least during the next coming years [Global Market Insights]. (Figure 5). 


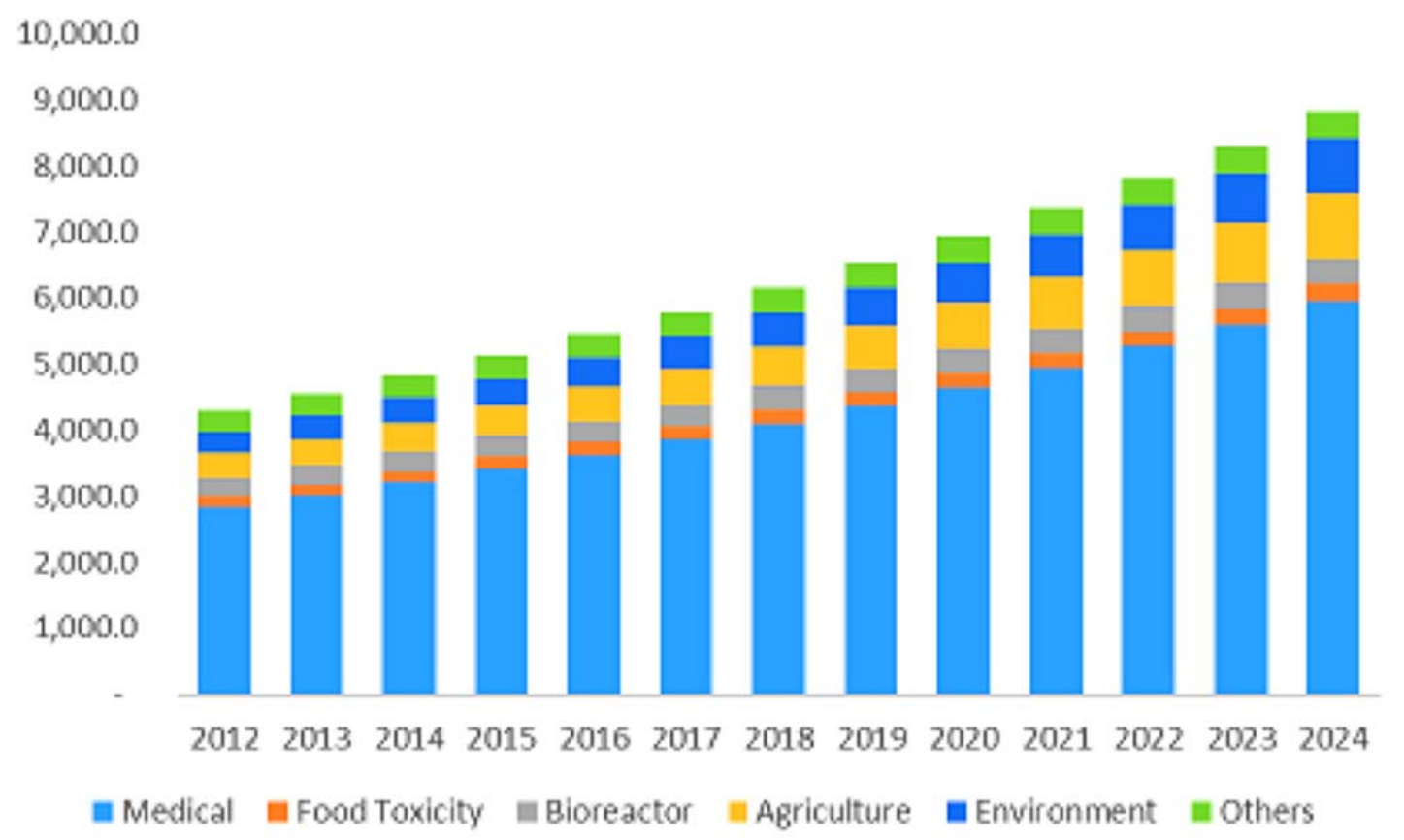

Figure 5: Applications of biosensors as a function of theism value in the market. From recent years to next upcoming years. .Biosensors Market Size, By Application, 2012 - 2024 (USD Million). [Global Market Insights].

Now a days there is taking place, a change in unprecedented clinical diagnose and treatment systems [Ortega, 2006] because, health and the improvement of the quality of life are issues of paramount importance for wealthy societies. That is the reason why medical and pharmaceutical industries are willing to invest in new clinical technologies covering the needs and concerns of the people, and also, because in the healthcare industry, the business is practically assured. [Cooper, 2002].

A biosensor must meet some requirements to really become the dominant technology of the commercial detection systems:

- High sensitivity

- Stability

- Low cost

- Possibility of miniaturization

- Capability to be included in integrated systems 
- Multiplexing

- Portability for in-situ diagnoses

\subsection{Types of biosensors}

At the present, there are several of biosensors typologies, all of them operating under the same principle, but combining different bioreceptors and transducing materials and signals. In this endless physical-chemical combination of their composition elements, the biosensors can be categorized in relation to different elements: Type of interaction of the biomolecules which can be biocatalitic (glucose sensor), or bioaffinity interaction (immunosensors, polymerase chain reaction or PCR). Type of bioreceptor: antibodies, enzymes, cells, nucleic acids and the type of transducer used to transfer the electrical signal into a quantifiable value. Lastly, they can also be differenced into labeled and not labeled biosensors. In label-free mode, the detected signal is generated directly by the interaction of the analyzed material with the transducer. In contrast, label-based sensing involves the use of a label on the biomolecules in order to intensify or amplify the transducing signal.

In this work biosensors are classified according the transduction methods they utilize.

\subsubsection{Classification of biosensors according to the transducing signal}

Transducer is an analytical tool, which provides an output quantity having a given relationship to the input quantity [IUPAC]. In biosensors it converts the biological signal reaction into a measurable signal. Some forms of transduction are: magnetic, electrochemical, piezoelectric (mass detection methods), micromechanical, thermal and optical detection. 
- Magnetic biosensors: Miniaturized biosensors detecting magnetic micro and nanoparticles in microfluidic channels. Using the magneto resistance effect has great potential in terms of sensitivity and manipulable size.

- Electrochemical: Best example of this kind of sensors is the glucose sensor afore described. In this type of biosensors, reactions between immobilized biomolecule and target analyte produce or consume ions or electrons, which affects measurable electrical properties of the solution, such an electric current or potential [Monosik, 2012], [Thevenot, 2001]. (Figure 6.A). They comprehend conductivimeters (impedance) [Guan, 2004], amperometric, potentiometric and also ion-selective biosensors.

- Piezoelectric: Those are mass-sensitive. Most popular are the quartz crystal microbalance (QCM), [Mannelli, 2003]. They measure the changes on the resonance frequency caused by a change of mass on the crystal structure quartz microbalance. Those are also called gravimetric sensors. Surface Acoustic Waveguide (SAW) are also commonly used and categorized in piezoelectric biosensors.

- Micromechanical: Microcantilevers show significant changes in two independent analyte-induced signals, resonance frequency and static bending, as the result of exposure to various chemical and physical phenomena. (Figure 6.A). Resonance frequency shift has the particular advantage of being relatively insensitive to interference from external factors such as thermal drift [Chen 1995]

- Thermal biosensors: Thermal biosensors or calorimetric biosensors study the biological reactions through the absorption or evolution of heat. [Ramanthan, 2001]. 
- Optical biosensors: In the optical biosensors the electromagnetic field (light source) interacts with biomolecular reaction on the optical transducer, this interaction is captured by a photodetector. [Fan, 2008] (Figure 6.A). They can be label-free optical biosensors will be further described in this work. Currently labeled optical biosensors are more commonly used in the clinical and medical industry. Due to their further develomentand and high reliability, most representative examples are: Enzyme-Linked Immunosorbent Assay (ELISA) or lateral flow analysis.

In figure 6.B it is showed the number of publications about the different types of biosensors from year 1985 to year 2010. The major increase of attention lies on electrochemical and optical biosensors, displacing other kinds such as magnetic or cantilevers. This is likely because, they are better responding to the technical aspects as well as the commercial needs afore-enlisted. This thesis is focused on the development of an optical biosensor.

(A)
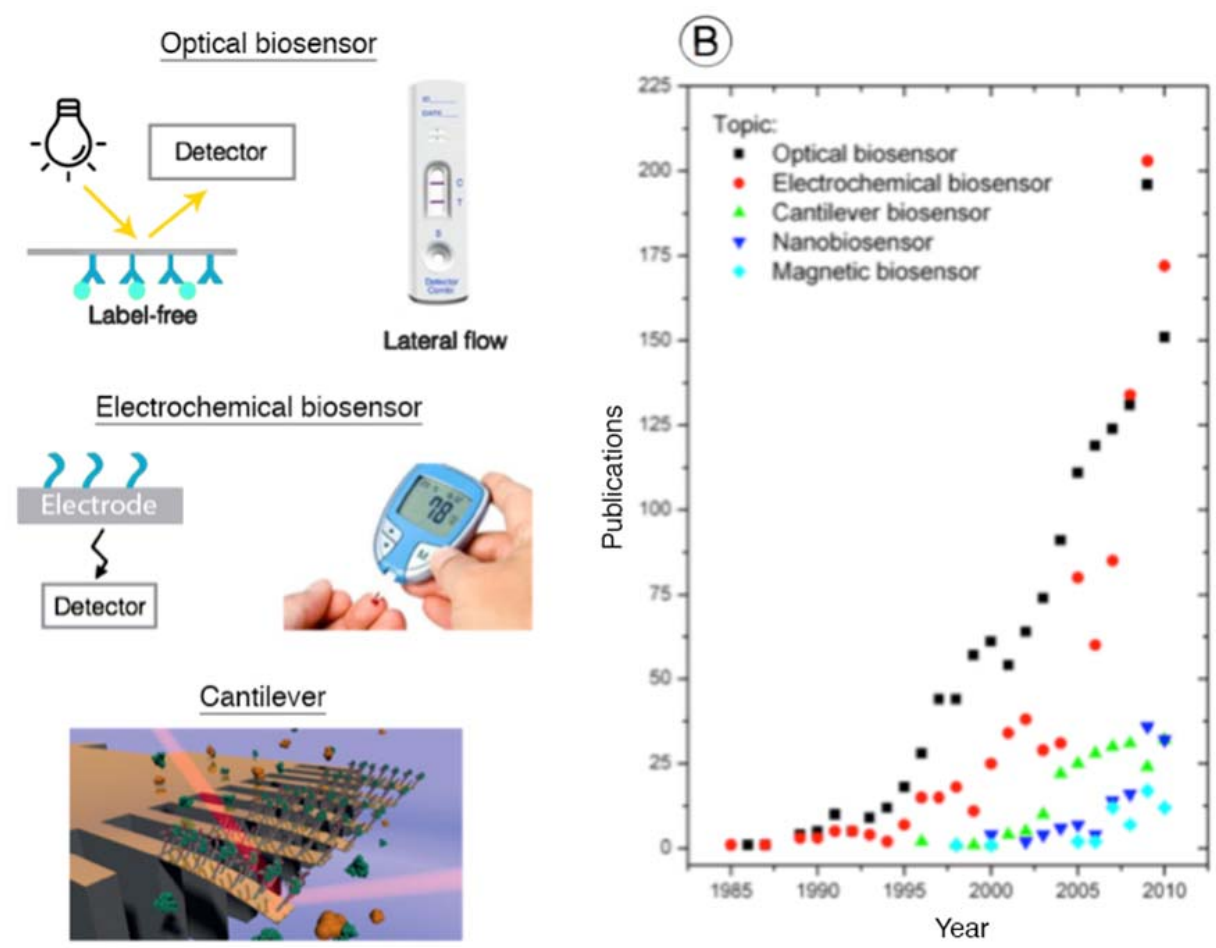

Figure 6: A. Basic example of optical, electrochemical and cantilever biosensors. B. Evolution of the number of publication per year for the different types of biosensors. 


\subsubsection{Bioaffinity Biosensors: Immunoassays}

In optical biosensors based on biomolecules affinity, the biorecognition element or biorecepctor needs to be immobilized on the optical transducer, to bind specifically to the target analyte and thus, convert the perceived signal into a comprehensible signal. The binding between the bioreceptor and the target analyte determines the degree of selectivity of the biosensor. Nucleic acid based biosensors (NABs) use nucleic acids as oligonucleotide probes (bioreceptor). They are daily used in hospitals for pathogenic infections detection and also in laboratory analysis for hybridization techniques and microarray systems. The better well-known technique using this kind of biosensors is the PCR (Polymerase Chain Reaction). Other biorecpetors used in bioaffinity sensors are aptamers, cell receptors, enzymes and also antigen and antibodies. When a biosensors is based on the the affinity ineraction between antigen and antibody (immunoassay), it is called immunobiosensors. The complex of antigen-antibody used for biosensor assessment is normally called bioapplication.

This thesis is focused on immunobiosensors based on immunoassays, which use antibody-antigen interaction for the detection of analyte.

Rosalyn Yalow and Salomon Berson first described immunoassays analyses technology in 1959 achieving limits of detection in the order of picograms for insulin biomolecules. [Berson and Yalow, 1959]. Since then immunoassays have been largely applied in different industries for molecules detection. It is a really powerful analytical tool very commonly used in optical biosensor. In this work, R-NPs biosensing capability is assessed through immunoassays.

The antigen ( $\mathrm{Ag})$ can be any type of biomolecule identified as an "estranger" by the immunological system, like viruses, bacteria, toxins, or some proteins. [de Juan, 2013]. When an "estrange" molecule is detected by the lymphocytes B (humoral response) they proliferate and differentiate to become plasmatic cells, main source of specific antibodies. [Gonzalez, 2005]. They synthetize and release the antibodies (Abs), which bind with a very high affinity and specificity to the antigen through a 
small available region of the antigen, called epitope. The lymphocytes $\mathrm{T}$ develop the immune cell response but their recognition of antigen is restricted to antigens situated on cellular bodies' surface; they also help lymphocytes B to start producing Ab.

The antibodies (Abs), also called immunoglobulin's (Ig) are glycoproteins (joined to sugars) belonging to the group of gammaproteinas. There are five main kinds or isotypes of antibodies: IgG, IgA, IgM, IgD e IgE. They differ from each other in function of the mass, shape, solubility and the antigen binding site. Each isotype is also subdivided according to the of sulfur bridges in the hinge region.

Immunoglobulin $\mathrm{G}$ is the most abundant antibody found in the bloodstream $(\sim 12 \mathrm{mg} / \mathrm{mL}$ in serum) and it protects the body from infection caused by bacteria, viruses and toxins (Figure 7). It is presented in the form of a monomer, with a Molecular Weight $\sim 150 \mathrm{kDa}$ and a size of $12 \mathrm{~nm}$ approximately. It has a Y-shape, formed by two identic fractions linked by disulfre bonds. Each fraction consists of a heavy chain $(\mathrm{H})$ with a mass of $70 \mathrm{kDa}$ and a smaller lighter chain (L) of $22 \mathrm{kDa}$. Each identical fraction is composed by a constant fraction (Fc) forming the base of the molecule, which corresponds to the carboxylic acid extreme, and a variable region consisting on the antigen binding fragment (Fabs) or paratope, corresponding to the amine group extreme. Between Fc and Fabs of the heavy chains there is a very flexible area (hinge area) for a better coupling to the antigens. Fab groups vary from one antibody to another regarding the specific antigen to be detected. Fc represents the major area of the heavy chain and is the binding region of the antibody to other proteins and cellular receptors. (Figure 8), [Janeway, 2001]. This is the area through which antibodies should be ideally attached to the transducer in the optical biosensors, leaving Fab areas available for antigen recognition. 

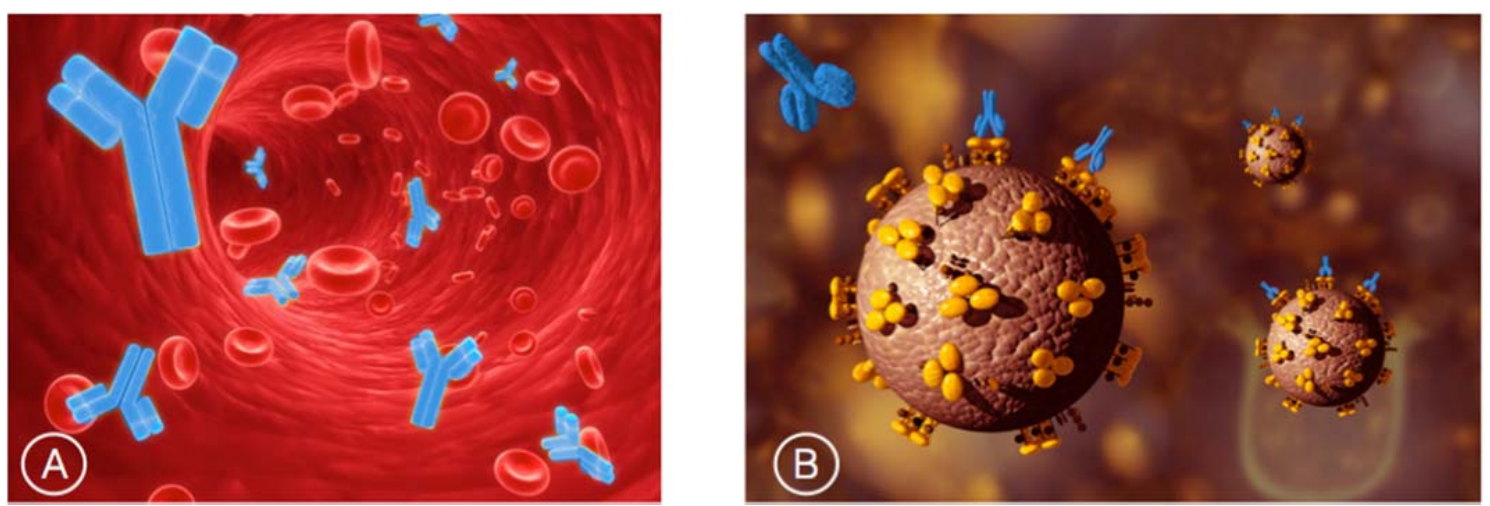

Figure 7: A. Antibodies travelling in the bloodstream. B. Antibodies recognizing specifically the epitope of the antigens [Guetty images].
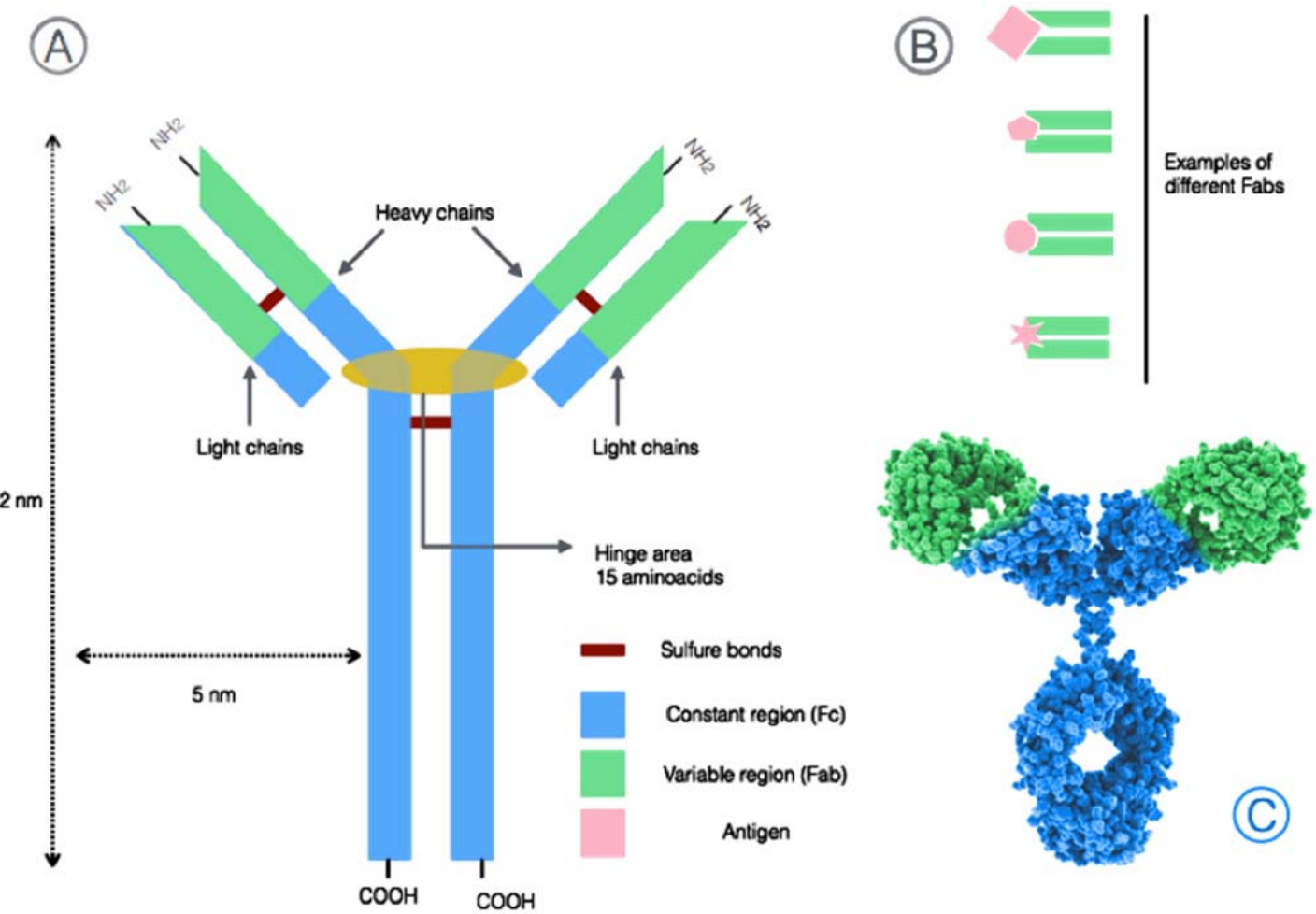

Figure 8: A. Scheme of antibody structure. B. Different Fab groups. C. 3D model of antibody conformation.

The antibody-antigen ( $\mathrm{Ab}-\mathrm{Ag}$ ) reaction is an equilibrium affected by several factors: temperature, $\mathrm{pH}$, ionic force of the solution, concentration of the reactive, incubation time, fluidic parameters (in the case of fluidic systems). To study coupling of $A g-A b$, there is a parameter used, known as dissociation constant $\left(K_{d}\right)$. This parameter considers the ratio of association of the bioconjugates (the 
reactive) as well as the disassociation rate of the product. Considering a reaction of $x$ proteins of antibody A with $y$ proteins of antigen $\mathrm{B}$, the equilibrium reaction results in [Casquel, 2012]:

$$
x A+y B \stackrel{k_{1}}{\leftrightarrow} A x B y
$$

$K_{1}$ defines the ratio of association of the reactive (Ag-Ab in this case) and $\mathrm{K}_{-1}$ represents the disassociation ratio of the conjugate (AgAb). In the equilibrium the $\mathrm{Ag}-\mathrm{Ab}$ reaction is equal to the AgAb complex, thus the relation between $K_{1}$ and $K_{-1}$ provides information about the affinity of $\mathrm{AbAg}$ conjugate, which is, the thermodynamic magnitude that points the interaction energy on a recognition point.

$$
\text { Affinity }=\frac{[A x B y]}{[A]^{x}\left[B^{y}\right]}=\frac{k_{1}}{k_{-1}}
$$

Commonly it is used the $K d$ which is defined as the inverse of the affinity:

$$
K_{d}=\frac{[A][B]}{[A B]}
$$

Then a small $K d$ implies a high tendency of the analyte to bind the antibody (high value of $K_{1}$ ), and a lower concentration is necessary to reach the saturation point between molecules

For immune bioapplications in biosensors, antibodies are produced specifically according to the antigen to be detected. There are tens of companies of biotechnology involved in the production of antibodies and bioapplications. The 
type of antibody that they produce depends on the customer bioapplication needs: affinity, specificity, purity...Antibodies can be polyclonal or monoclonal.

Polyclonal antibodies: (pAb) For their production, an animal is stimulated with an immunogen (antigen) and as a response the animal produces a heterogeneous great quantity of Igs against the different components of the antigen (peptides, polysaccharides). Polyclonal antibodies can react with different epitopes of the antigen. Their production cost is low, fast (around three month) and reproducible from one batch to another. They are very sensitive and more tolerant to changes in proteins configurations.

Monoclonal antibodies (mAb): Unlike polyclonal antibodies, they can only react with one single epitope of the antigen. They are produced at a low throughput, taking long periods of time (12 month). In monoclonal antibodies production, all the Igs are exactly the same and can be easily reproduced. They are less sensitive but more they have more specificity. [Killard, 1995].

In figure 9 it is represented a very schematic procedure of obtaining both types of antibodies.

\section{Polyclonal Abs}

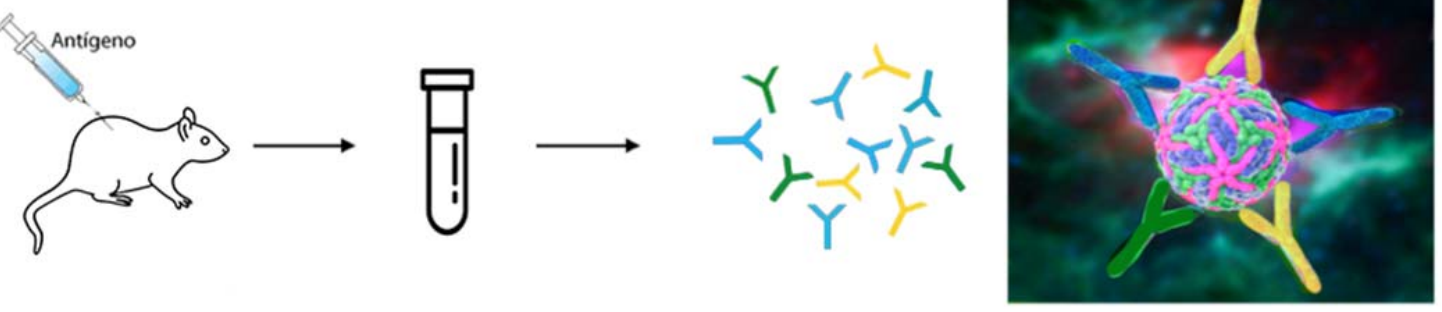

\section{Monoclonal Abs}

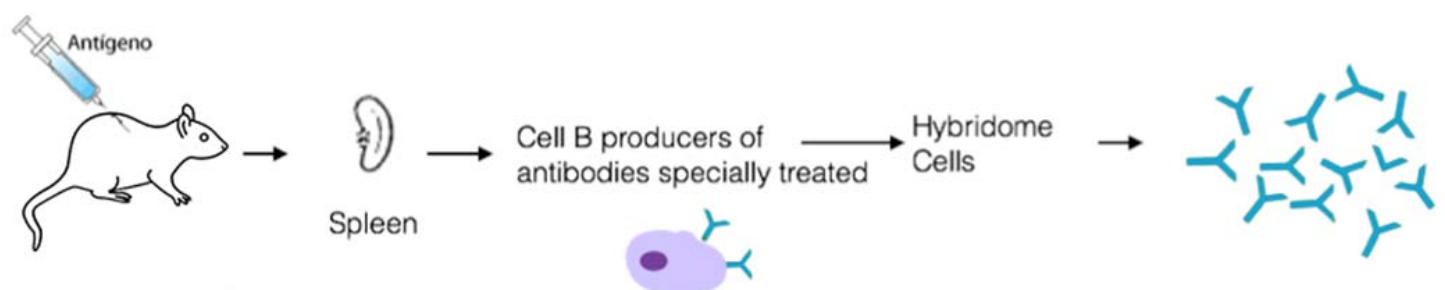

Figure 9: Procedure of production of polyclonal antibodies and monoclonal antibodies. The picture shows polyclonal antibodies recognizing different epitopes from an antigen. 
Also in the last years industry is focusing the antibodies production, on the development exclusively of the antigen binding areas, according to the final applications. For example now a day it is possible to obtain only the Fab area, assuring the availability of the antigen binding sites, for analyte recognition, and therefore improving biosensing performance.

\subsubsection{Types of Immunoassays}

In biosensors research field, the immunoassays are used to evaluate the real capacity of the biosensors to detect a biomolecules. They consist in two basic stages: Immobilization o the bioreceptor (antibody or antigen) and the recognition of the antigen or the antibody, is case the antigen had been used as bioreceptor. There are several types of immunoassays. (Figure 10).

- Direct Immunoassay: In this kind of immunoassays the antibody is immobilized on the surface of the transducer until total saturation. When this is fully accomplished, the biosensor is ready to recognize specifically the antigen. There is a special type of direct immunoassays called "sandwich immunoassays" in which to the complex antibody-antigen it is added a secondary antibody for the antigen binding. Thus the antigen is trapped between both antibodies. This is a good strategy used for small molecules detection, because with the addition of the secondary antibody the molecular mass of the whole complex is increased and normally is easier to detect. In labeled assays, the secondary antibody is labeled with a substrate, providing signal intensity as a function of the quantity of antigen present.

- Indirect Immunoassay: For some diseases detection is preferable to control the presence of the antibodies in the serum samples and thus infer the type or stage of the infection. For this purpose is necessary to immobilize the antigen over the transducer surface. Therefore the biosensor can be able to detect specific antibodies, which are acting, as the analyte is this type of immunoassays. 
- Competitive: In competitive immunoassays there a molecule (called competitor (or analyte in this type of immunoassays), which is very similar to the antigen, is immobilized on the surface. Then, a sample containing the antibodies and the antigents in brough in contact with the immobized surfaced with the competitor. During the incumbation, the analyte or competiror and the antigen, compete for the antibody binding. The antigen binds the antibody, hampering the recognition of the analyte immobilized in the surface. Thus in this kind of immunoassays, less detected signal implies less attached antibody and therefore more antigen (target molecule) in the tested sample.
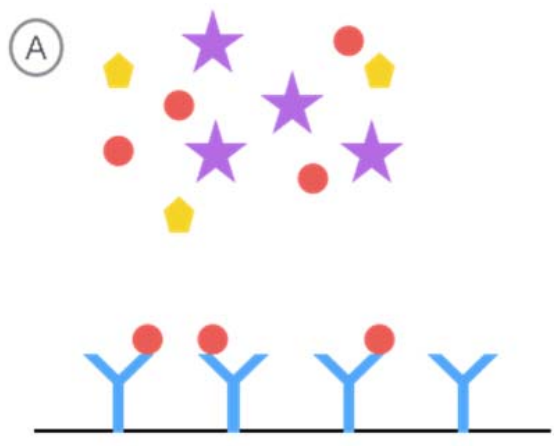

Direct Immunoassay

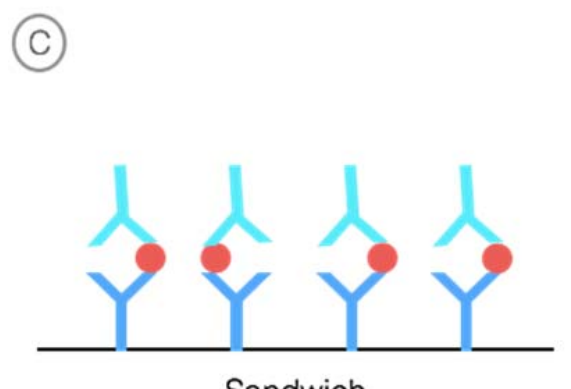

(B)
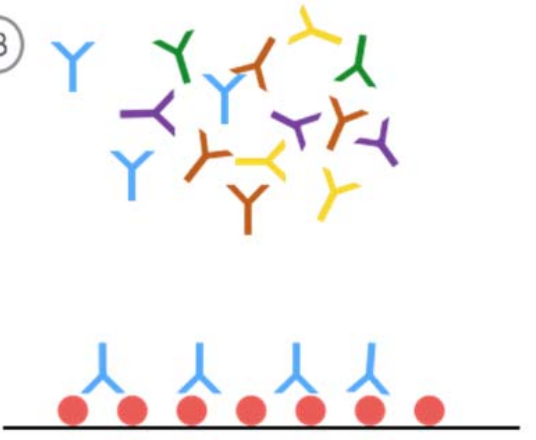

Indirect Immunoassay

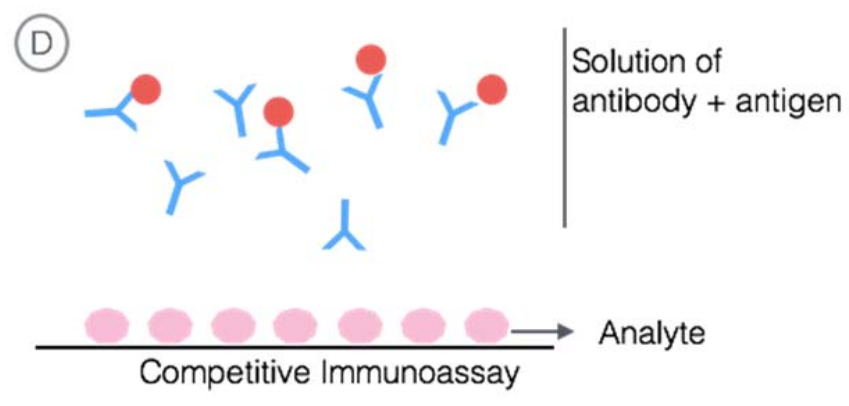

Figure 10: Types of immunoassays. A. Direct Immunoassay. B. Indirect Immunoassays. C. Direct Immunoassay: Sandwich. D. Competitive Immunoassay. 


\subsection{Optical Biosensors}

Label-free optical biosensors are experiencing an exponential development and attracting the interest of many different sectors. They operate at a physical scale unreachable until the last decades in which new technologies have unblock the widespread of the knowledge and use of this kind of sensors. (Figure 11).

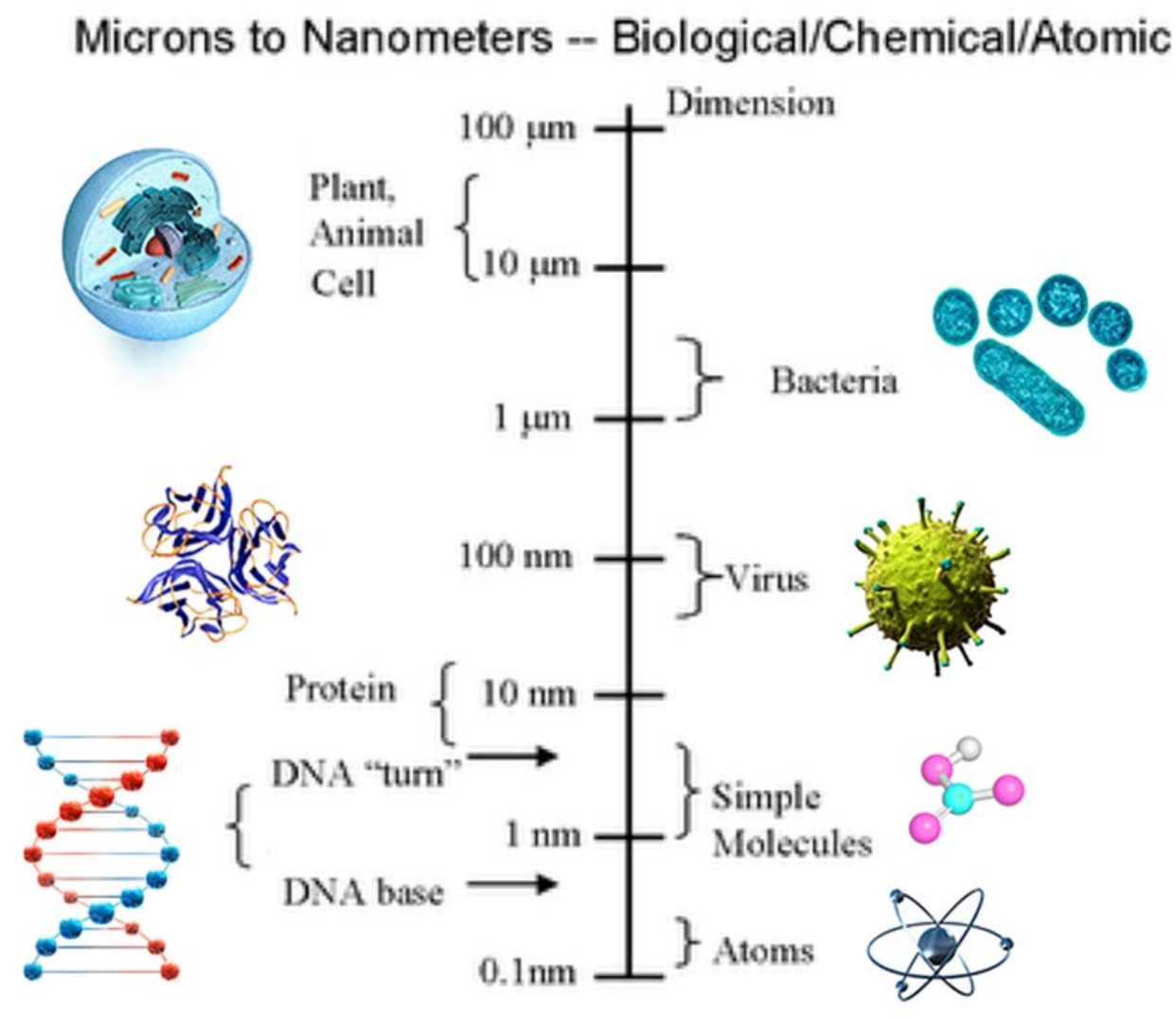

Figure 11: Size scale in which optical biosensors operate.

Most of biosensors can be label-free or label-based. In label-free mode, the detected signal is generated directly by the interaction of the analyzed material with the transducer. Unlike this, labeled biosensors need a molecule reacting with one of the species participating in the biological process in order to increase or produce a signal easily noticed or measurable. 


\subsubsection{Optical Labeled Biosensors}

There are some biological species that due to their low mass or size, are extremely hard to quantify. Besides, sometimes they are present in samples in such a low concentrations as femptograms/L. For those reasons, conventionally they need to be labeled in order to amplify the signal of the biological binding, and therefore allow and enhance the detection and quantification of certain analyte. As labels there can be used, radioisotopes for radioimmunoassay's, fluorescent molecules for fluoroimmunoassays, enzymes in enzimeimmunoassays, luminescence particles or a nanoparticle among some others.
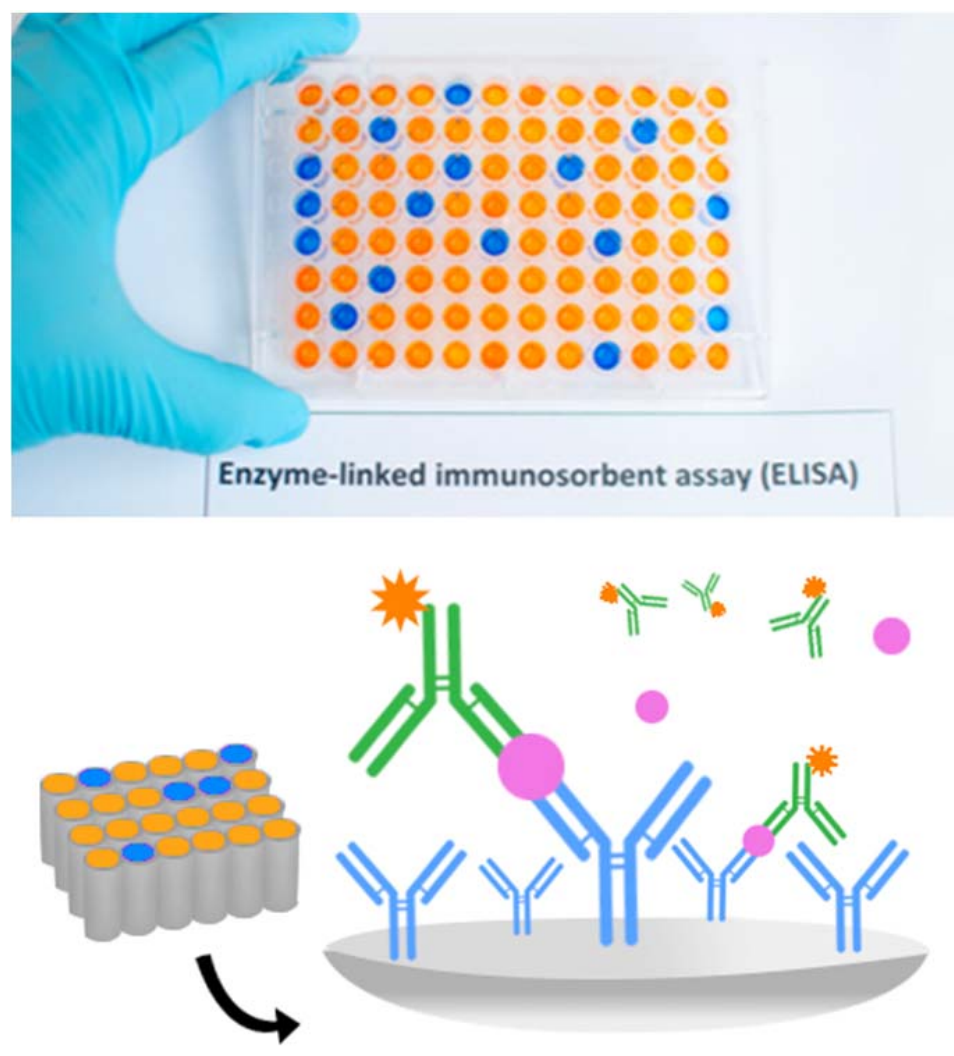

Figure 12: Upper picture: Output of ELISA plaque with 96 microwells. Below, scheme of a sandwich immunoassay with labeled secondary antibodies. [Getty images].

Enzyme-Linked Immunosorbent Assay (ELISA) (figure 12) is commercial detection test normally used for clinical and research applications. It consist on a plate containing 96 polystyrene microwells, each of them hosting the adsorption of 
the bioreceptor for the analyte detection in the solution. The analyte is detected by labeling secondary antibodies with an enzyme able to produce a change in the color solution if the immunoreaction takes place. Horseradish peroxidase (HRP) substrate is very commonly used. Different immunoassays typologies can be developed on the microwells but always with some sort of label. For the quantification of the analyte in the different wells, ELISA plaques are analyzed by spectrophotometry [Lequin, 2005].

It is a routinely quantitative process that provides high detection sensitivity and reliability, but normally it is a long and expensive process that requires large amounts of sample, specific equipment and human expertise to be performed.

Other popular detection test, based on molecules labeling is the Lateral Flow Test. (Figure 13). One example of this analytic tool is the lateral flow used for the detection of the human chorionic gonadotropin in pregnancy test. This is a qualitative or semi-quantitative analytical tool.

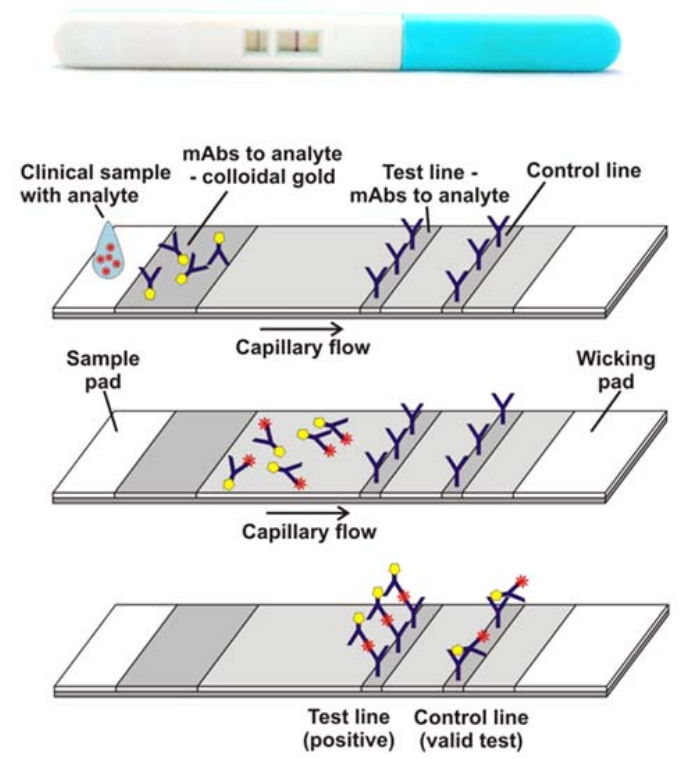

Figure 13: Commercial pregnancy test. Explanation of the operating of lateral flow test. [DxDiscovery].

Currently the diagnostic systems must be compared, considering their cost and complexity as a function of their quality or performance. The ELISA test, the 
current gold standard biodetection system carried out in the laboratories, is expensive in comparison to the qualitative lateral flow devices that offer a lower performance at a reasonable cost. The challenge is to develop devices capable of offering the same or better quality as ELISA tests, but to a price at the level of lateral flow devices. (Figure 14). A good example nowadays is the glucose tests that comply with the requirements of an ideal PoC diagnostic system.

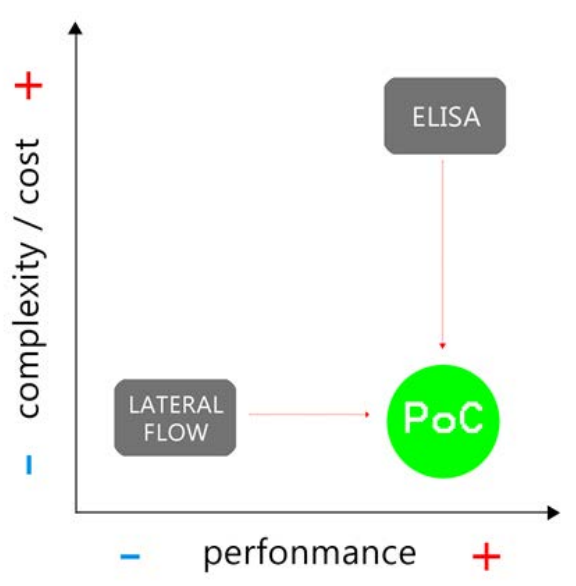

Figure 14: Ideal Point of Care detection system must have a high performance at a low cost.

\subsubsection{Optical Label-free Biosensors}

Labe-free test formats are performed without any labelled compound. When the analyte binds to the transducer through the bioreceptor, it produces a change in the measurable response; in the case of optical biosensors it is an optical response. Their simplicity in operationally have lead them to a potential tool to complement or even displace other detection technologies by focusing specially in the development of integrated optical biosensors (IO) of high sensitivity. [GonzalezGuerrero, 2016]. Here after are described different types of optical label-free biosensorsaccording to their underlying principles. [Cooper, 2009]. 


\subsubsection{Surface Plasmon Resonance (SPR)}

Surface Plasmon Resonance (SPR) biosensors are refractive index (RI) based optical sensors, studying surface plasmon to probe interactions between an analyte in solution and a biomolecular recognition element immobilized on the SPR sensor surface. [Homola, 2003], (Figure 15). The surface of a SPR is made by an interphase between a thin film of dielectric material and a metal layer material, normally gold. The incidence of the plane polarized light over the surface through the prism generates Electromagnetic Wave (EW) that is propagated through the boundary of the interphase. This propagation is called Surface Plasma Wave (SPW) or plasmon. [Sweccha, 2016].
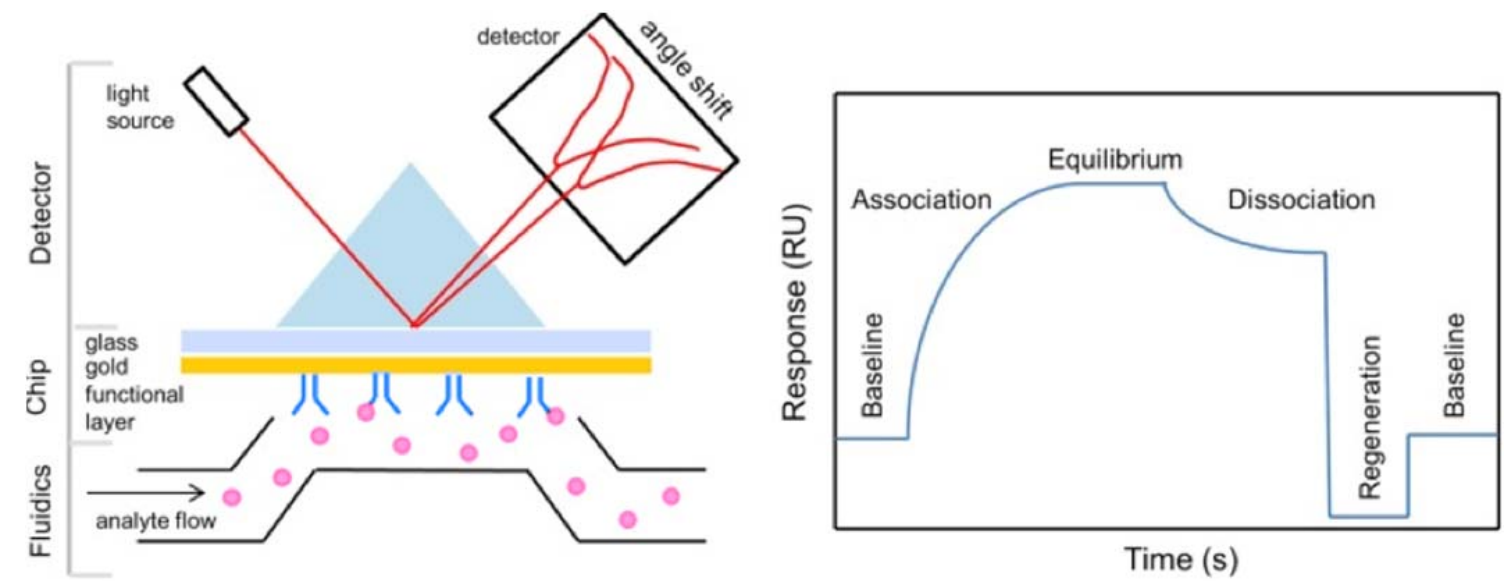

Figure 15: Basic scheme of SPR operationally. On the right, SPR sensogram, showing the steps of an analytial cycle. [Damborský, 2016].

The intensity of the plasmon is maximum at the interphase. The attached molecules (acting as the dielectric material) to the surface (metal part) can change its Refractive Index (RI), producing a change in the propagation constant and thus, altering the coupling of the plasmon surface and the incident light. By tracking the changes of the characteristics of the optical wave it can be inferred the presence or the concentration of a target analyte. [Homola, 2006]. They can be performed in real time, so kinetics details of the interaction between target analyte on the solution and bioreceptor immobilized on the metal surface, can be determined as they happen. They can measure from proteins, oligonucleotides, oligosaccharides, and lipids to small molecules, phage, viral particles, and cells. [Rich, 2000]. 
Recently a variant of this type of biosensor is being studied. Localized Surface Plasmon Resonance (LSPR) is used for very small molecules detection, being the molecules smaller than the wavelength of the incident light. The plasmon oscillates locally around the molecule to detect. The LSPR wavelength strongly depends on the dielectric properties in the vicinity of the nanoparticles, thus; the biosensing based on LSPR is accomplished by measuring the shift in LSPR wavelength due to the adsorption of biomolecules on the surfaces of nanoparticles. [Sepulveda, 2009], [Willets, 2007].

SPRs have a very high sensitivity in which the smallest change in the refractive index measured in the sensor is in the order of $10^{-7}$ RIU (Refractive Index Units) for table-top laboratory systems and of the order of $10^{-6}$ RIU for portable SPR sensors. [Homola, 2012]. SPR normally works in continuous flow, real-time systems, allowing thus the study of kinetics of the biomolecules binding events such as affinity or specificity. However, precisely their need to operate in continuous flow systems hinders their true development as PoC detection systems.

SPR-based biosensors were proved in 1983, since then they have become one of the most used label-free biosensors for commercial purposes, especially by GE Healthcare Co. (Biacore), Sensia S.L., Bio Tul AG, Windsor Scientific Limiteds or Texas Instruments.

\subsubsection{Interferometric sensors}

\subsection{Mach Zhender}

It is an interferometric biosensor in which light beam is split in two beams that travel through two equal but independent waveguide paths. One path is exposed to the sample to analyze, and the other is used as a reference. (Figure 16). Then, the paths come together again and the change of the light phase can be studied. Evanescent field crossing the waveguide path within the biomolecules attached will notice a change in the refractive index so, the optical length will change 
regarding to the reference path, there will be a delay between the evanescent fields of both paths. [Zetier, 2000], [Prieto, 2003].

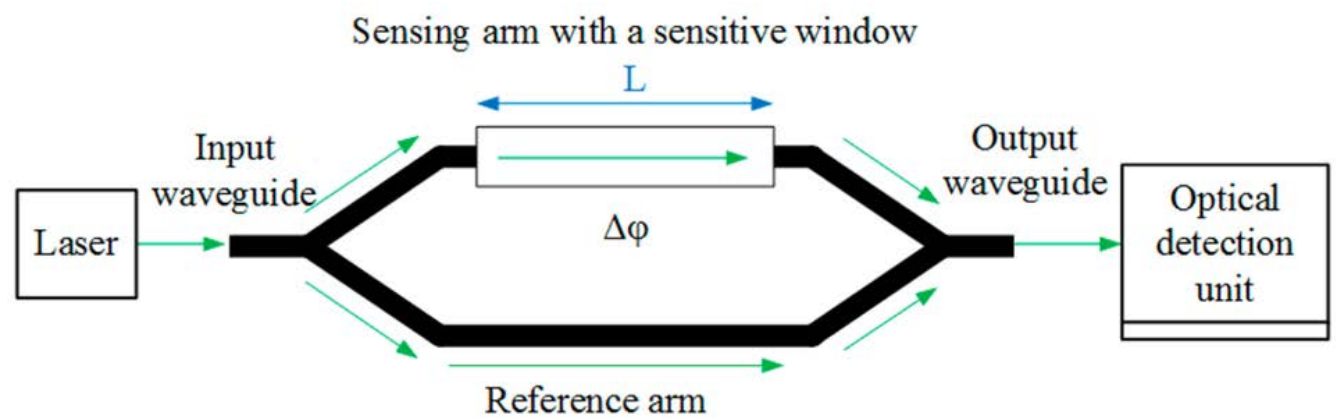

Figure 16: Mach Zehnder scheme. [Yuang, 2015].

Signal output shows the change on light phse as a function of the refractive index variation. Its biosensing capability has been demonstrated in the literature, by the detection of biotined-BSA and streptadivin [Luff, 98].

\subsection{Dual Polarization Interferometer (DPI)}

DPI was developed by Cross and collaborators [Cross, 2003] and is composed by two thin films acting as waveguides, one above another. The one on the top is in contact with the sample test and the film below remains in contact with the chip surface. Between them there is a dielectric material of lower RI. When light is coupled into the waveguide it can be observed an interference pattern corresponding to Young interferometer. It can be studied the tranvers electric (TE) and tranvers magnetic (TM) modes independently, obtaining thus accurate values of the RI and the thickness of the layer. 
2.4.2.3 Biosensors based on optical cavities

\subsection{Ring Resonators}

Ring resonators are are ring-shaped disk (solid medium), coupled to a waveguide. [Boyd, 2001]. It is the formed a cavity resonance between ring and the waveguide. Biomolecules are attached to this solid medium producing a spectral shift in the cavity resonance wavelength, which is directly proportional to the total mass of bound molecules per unit surface area. [Iqbal, 2010]. (Figure 17).
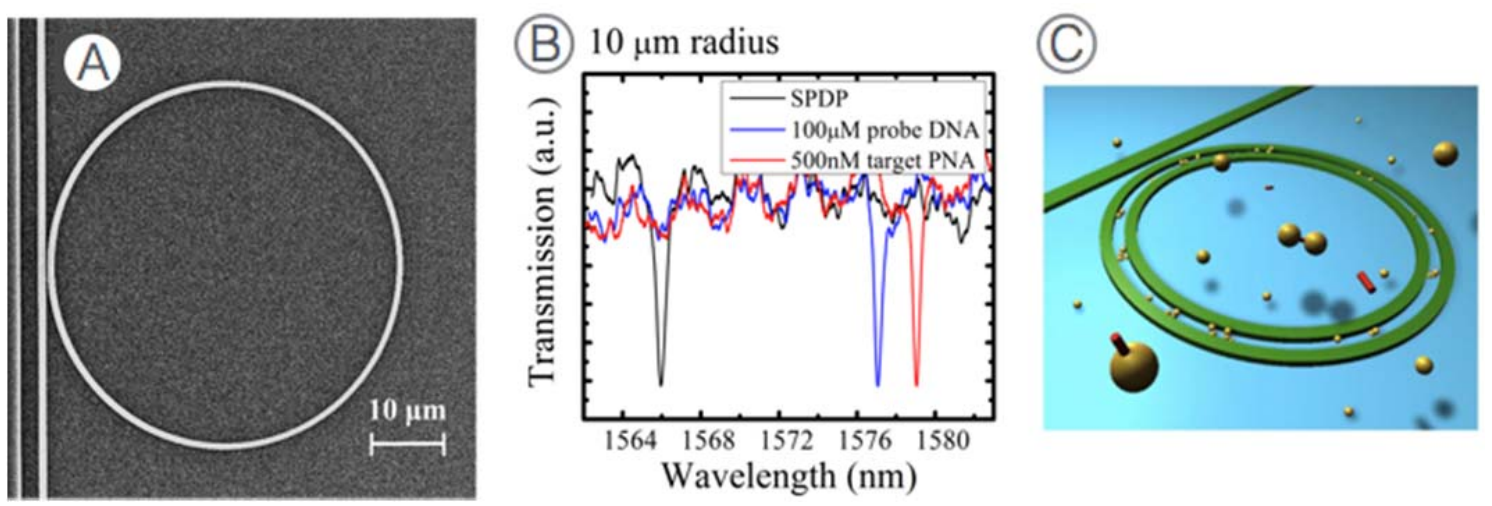

Figure 17: A. SEM image of a silicon ring resonator. B. Cavity resonance mode shift for biomolecules detection. C. Scheme of a waveguide slot. [Witzens, 2011].

The waveguide surface has an evanescent field, which is exponentially decaying into the surrounding medium. Therefore the resonant wavelength is affected by the refractive index of the solution and/or biomolecules in contact with the waveguide surface. [Yangqing, 2010].

In addition it can be studied the effect of the light coupling between the waveguide and the ring. More molecules on the surface imply increased light coupled in the disk.

Ring resonators, also called disk resonators have a very high Quality factor, (Q-factor), and can be integrated in lab-on-a-chip sensing devices, 
[Carlborg, 2010], although, their fabrication is complex, involving sophisticated fabrication techniques.

Also some authors have studied slot waveguides (Figure 18.A) for the detection of small changes in the RI from different fluids, [Barrios, 2007]. They are composed of two waveguides, leaving a low RI area (slot region) in between through which light is confined. (Figure 18.B). In a latter work of Barrios it was demonstrated the sensing performance of this slot waveguides by measuring Bovine Serum Albumin (BSA) and its corresponding antibody (anti-BSA). [Barrios, 2008].
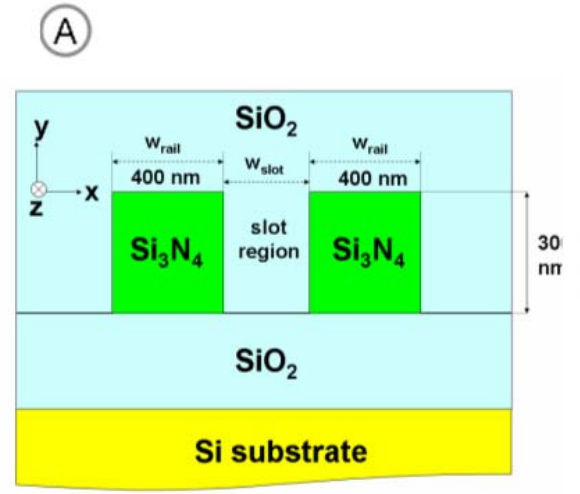

(B)

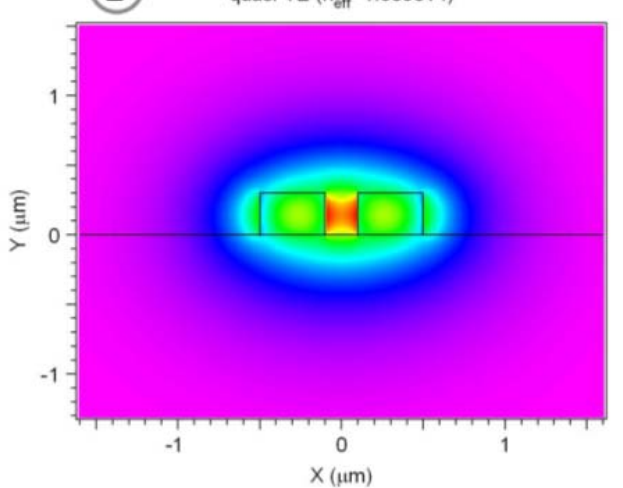

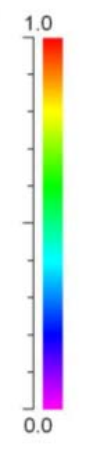

Figure 18: A. Cross view of a waveguide slot scheme. B. Simulation of light confinement in a slot waveguide. [Barrios 2007].

\subsection{Photonic Crystals}

Photonic Crystal (PC) are composed by periodic dielectric materials nanostructured arrays acting as a mirror for a specific range of wavelengths, which is called photonic band gap (PBG). The nanostructures alternate dielectric periodic materials of lower and higher RI, disposed in 1, 2 or three dimensions. [Domínguez, 2013], [Robinson, 2013]. By introducing a defect or a cavity in the array, the light can be transmitted in a very narrow spectral range, forming a sharp resonance mode in the optical response. (Figure 19 A and B). 
When the biomolecules are attached to the surface, the resonance mode is shifted. (Figure 19.C), [Chow, 2004]. Eevnthough photonic crystals have showed to present high Q-factors their fabrication process results a critical issue for their popularization.

(A)

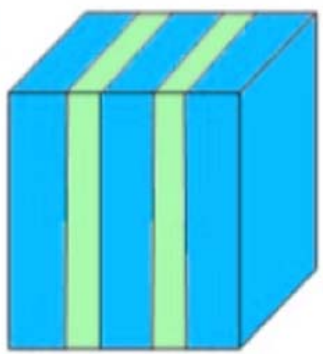

1D-periodic in one direction

(B)

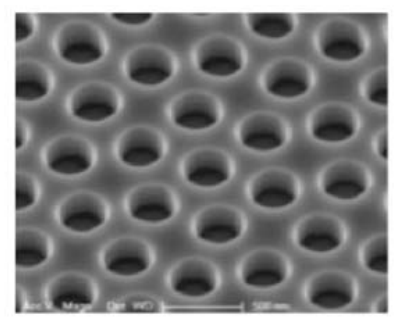

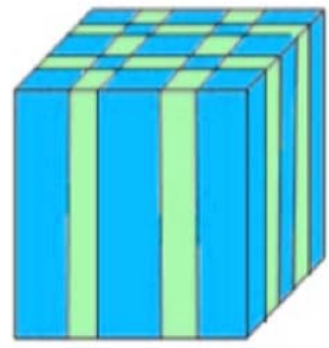

2D-periodic in two direction

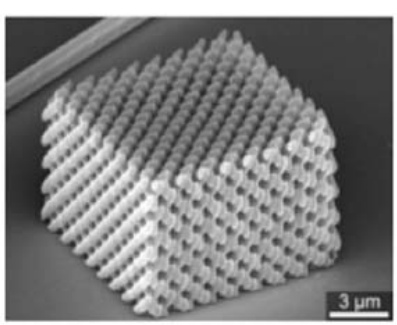

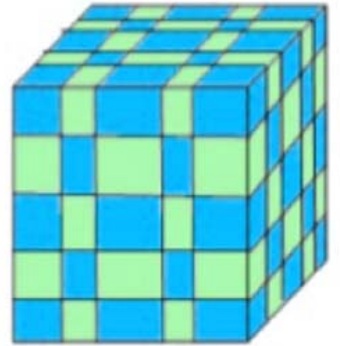

3D-periodic in three direction

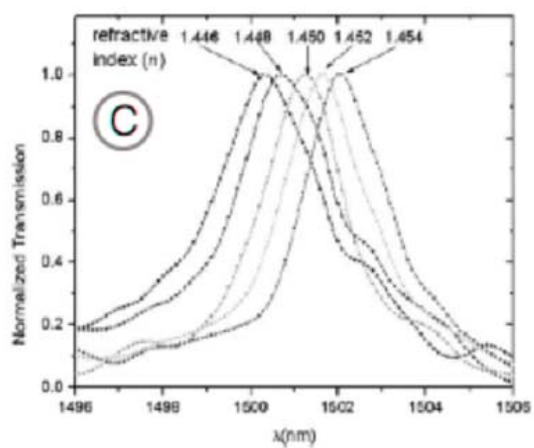

Figure 19: A. Different dimension of PC. B. SEM pictures of 2D and 3D. C. Resonance mode for different RI fluids measured with PC. [Robinson, 2013]. [Chow, 2004].

\subsection{BICELLS}

BICELL is the acronym of "Biological Sensing Cell". These are RI interferometers based on nanostructures arrays distributed along the substrate, that are vertically interrogated. This avoids the long complex process of light coupling that takes place in waveguide biosensors. In addition, BICCELLs permit straightforward analyte infiltration and liquid trapping. [Lopez-Romero, 2010]. For the vertical interrogation of the nanostructures it is used a microscope (to allow positioning in the array) with a light source (striking the whole array area) connected to a spectrometer. Then, specific data management software, shows the optical 
response of that array by representing the signal intensity of the nanostructured array as a function of the wavelength according to the spectral range of the spectrometer and the light source.

Holgado and collaborators demonstrated the biosensing capacity of BICELLs based on nanopillars lattice fabricated with SU-8 polymer, by detecting increasing concentrations of BSA/anti-BSA bioapplication, reaching limits of detection in the order of ng/mL. [Holgado, 2010].

The nanostructures can have different geometries and materials. Moreover different test have been accomplish to this regarding. (Figure 20). Doctor Sanza fabricated and analyzed the performances, of several nanostructures geometries made of SU-8 resist [Sanza, 2015], and compared them with flat layers of SU-8 acting as simple Fabry-Perot interferometers. Better performances in terms of sensitivity were found for nanopillars of SU-8, due to the grater light confinement among the nanopillars, increasing therefore, the sensitivity, of the BICELLs.
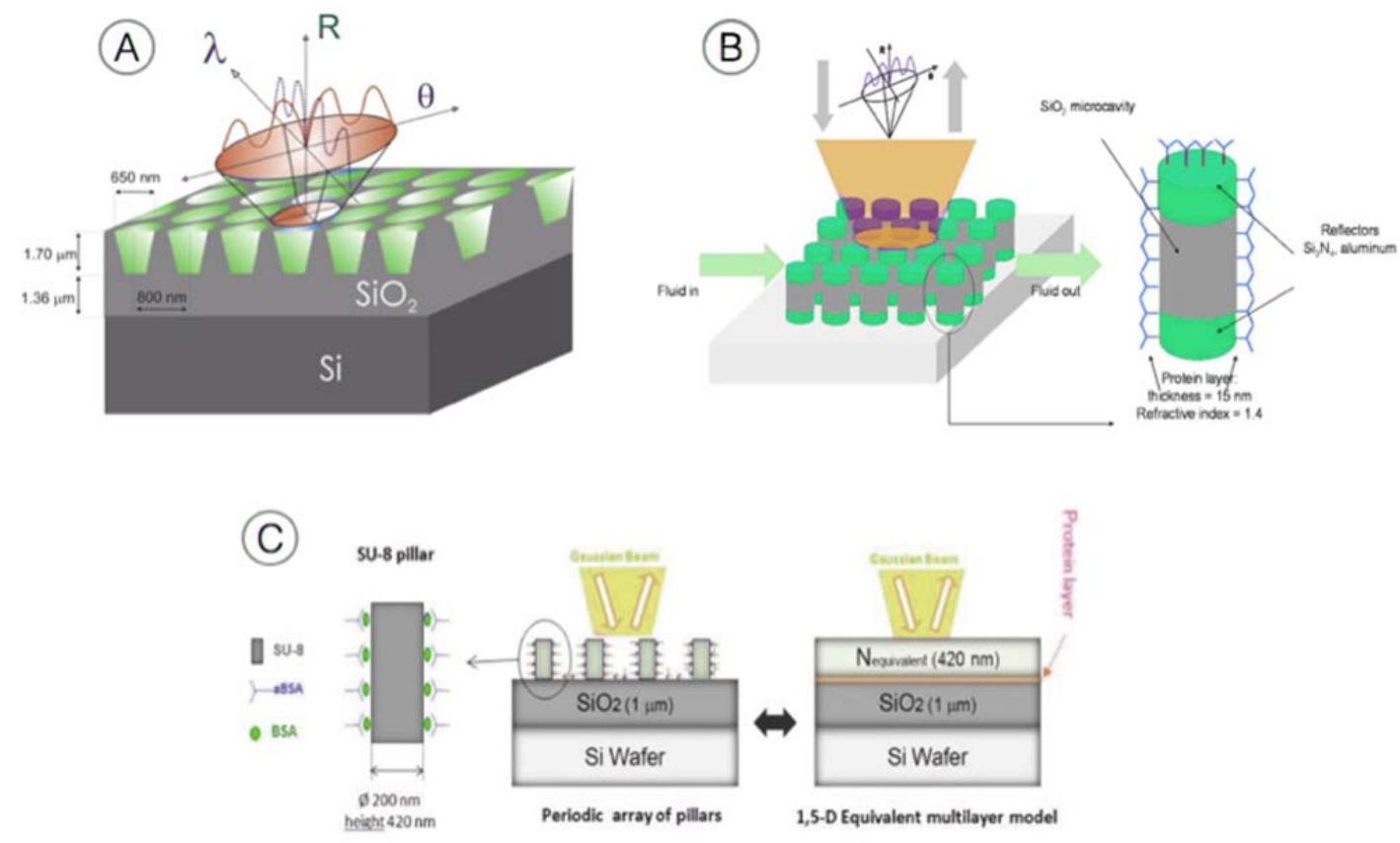

Figure 20: A. Array of holes fabricated over a $\mathrm{SiO}_{2} / \mathrm{Si}$ Wafer. [Holgado 07]. B. Resonant nanopillars. C. SU-8 nanopillars over $\mathrm{SiO}_{2}$, and theoretical model for RI calculation. [Casquel, 2012]. 
Resonant Nanopillars (R-NPs) studied in this work could be included in this group of label-free biosensors (BICELLs). (Figure 21). Each nanopillar consist of several $\mathrm{SiO}_{2} / \mathrm{Si}_{3} \mathrm{~N}_{4}$ Bragg reflectors pairs over a quartz surface and a central cavity of $\mathrm{SiO}_{2}$. They are arranged into independent arrays that are vertically interrogated from the backside. When R-NPs arrays are biofunctionalized they can be called BICELLs, nevertheless each single resonant nanopillar could be also considered as a PC 1D.

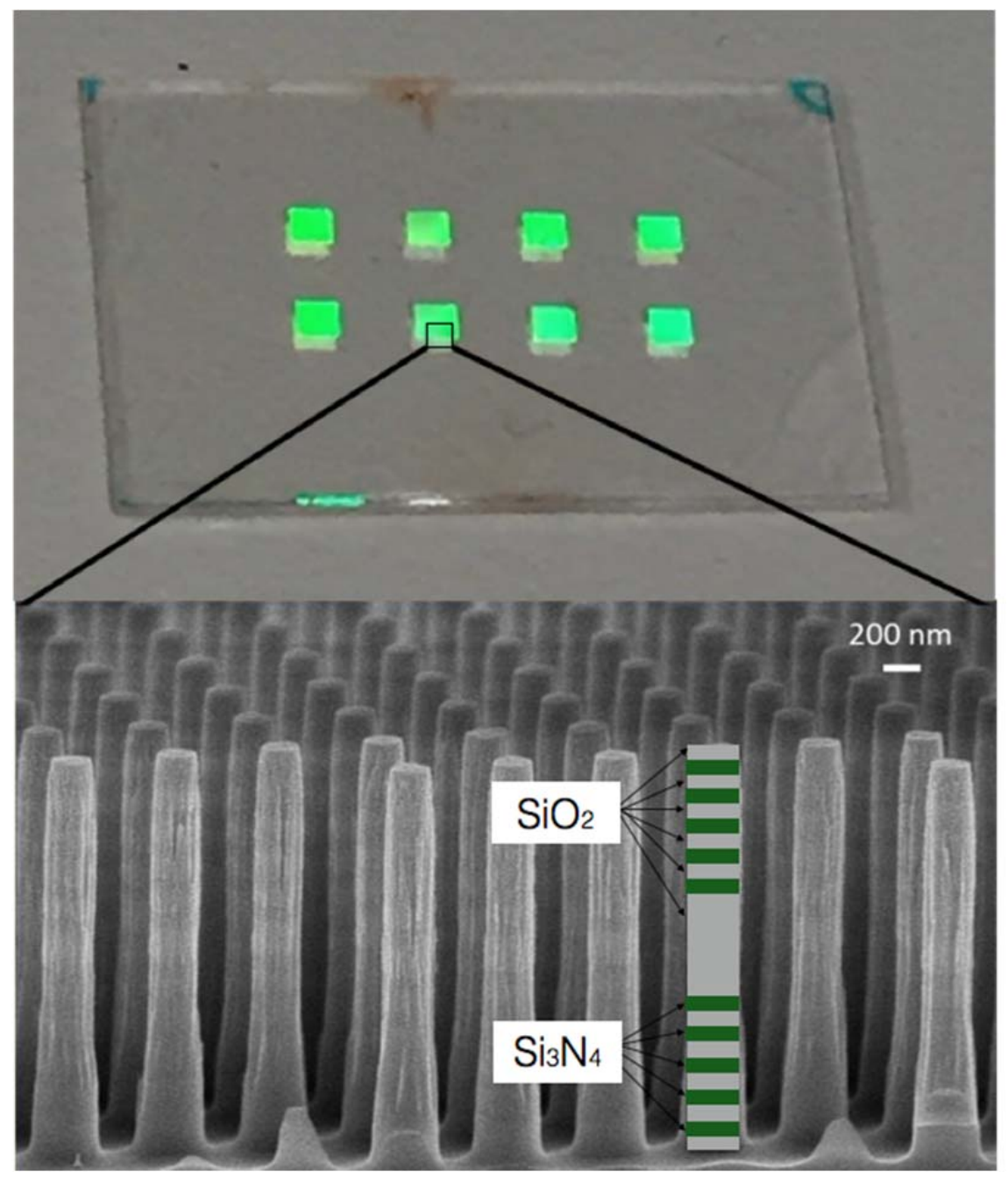

Figure 21: Upper picture: Example of a chip with BICELLS of R-NPs. Down, SEM image and scheme of R-NPs.

Discoveries of new materials, nanotechnologies and the exponential increase of transferred knowledge have been indisputable elements for the development of afore described label-free biosensors. They have demonstrated high performance, 
providing good sensitivity values under laboratory conditions. In fact even some research lines are heading to single non-labeled molecules for ultra-sensitive biosensing. Even though, they still have some pending subjects to overcome in order to become a commercial product. [Zancheta, 2017]. First of all they need operationally and simplicity where not many steps are involved, and no expertise's are need to make a proper use of them, when measuring real samples in-situ (calibration, sampling measuring and interpretation). Also they need to be portable, appealing to lab-on-a-chip and Point of Care devices. Lastly, for commercial success it is essential that they are cost-effective products, made of non-expensive materials, easy to find in the market, so biosensors or at least the transducers can eventually be disposable.

Some research groups are working at the moment on the concept of "biosensors low cost" in which affordable materials and designs are the base of their development. In figure 22 there are shown some examples.

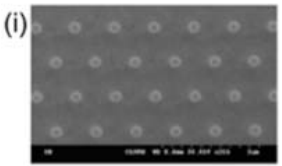

(ii)

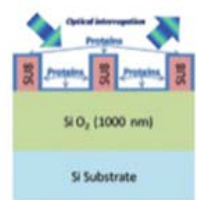

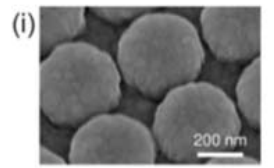

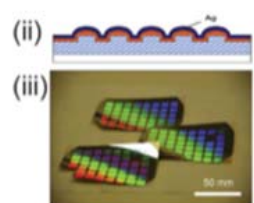

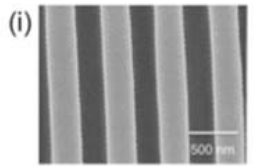

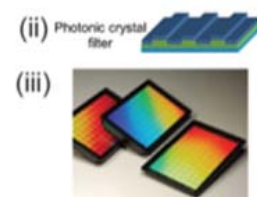

(i)
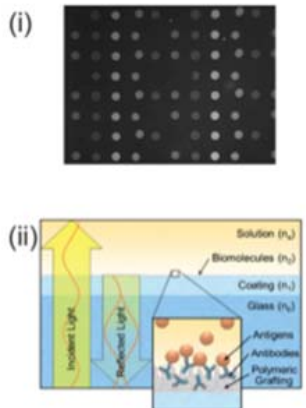

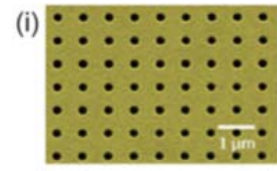

(ii)

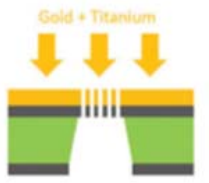

Figure 22: Photonic structure made by nanopillars of $\mathrm{SU}-8$ resist on $\mathrm{SiO}_{2}$. (i) $\mathrm{SEM}_{\text {image and }}$ (ii) schematic representation. (B) Photonic structures formed by Ag-coated nano-domes on flexible polyethylene terephthalate (PET) sheets. (i) SEM image; (ii) cross-section diagram; and (iii) image of the complete array substrate. (C) PC resonant reflector made by TiO2 -coated UVcurable polymer. (i) SEM image; (ii) schematic of the substrate; and (iii) image of the array in standard microplate format. (D) RPI sensing surface made by SiO2 anti-reflective layer on a

glass slide. (i) RPI image of antibody spots with diameter of 150-200. [Zancheta, 2017].

\subsubsection{Biofunctionalization techniques}

In the last subchapter different biosensors typologies have been studied from a physical point of view. There have been described different materials and 
morphologies that can be applied for the transducer, and also different manners of optical interrogation of the sensors have been shown. Even though it is of paramount importance to bear in mind that, however cheap is the material used, however efficient is the interrogation method or however susceptible to be miniaturized is the biosensor, it is pointless if the transducer cannot be immobilized with an effective, stable, reproducible and homogeneous layer of bioreceptor.

The immobilization process is called biofunctionalization and provides the sensor its biosensing capacity. When the transducer is covered with the bioreceptor it can be said that the surface is biofunctionalized, and is ready to bind specifically to the target analyte to be detected.

According to the bioapplications to be measured, the process of biofunctionalization must be adapted. Ideally it should consist on few steps, covering the surface homogeneously in a compatible way with the optical properties of sensing surface. Ideally, biofunctionalization should be reproducible, avoid non-specific binding (fouling) and minimize the reagent volumes involved. It is a critical phase that can definitely affect to the biosensor performance. Some unsuitable events can occur during biofunctionalization process that must be avoided: physical or chemical damage of the biomolecule, affecting its conformation and affinity properties, nonspecific binding, randomness in the orientation, blocking the reactive binding groups, inhomogeneity along the surface and matrix effect of the biorreceptor's containing sample. [Bañuls, 2013].

There exist different approaches for biomolecules immobilization over sensors surfaces:

\subsubsection{Physical Adsorption}

Physical adsorption of biomolecules over surfaces is fast, but a relatively weak method of immobilization. It can be applied to porous or hydrophilic materials, but the binding is not stable either in time or along the surface, because biomolecules 
are attached to the surface through electrostatic forces, depending on electrical charges. If the charges change, the binding can be easily braked down.

For an optimal biofunctionalization process is desirable to create strong covalent bond between inorganic surface and organic molecules.

\subsubsection{Molecular Imprinted polymers (MIP)}

This method is a very selective method used in biosensors. It is focused on the "printing" of functional groups of the bioreceptor into a polymer. (Figure 23). These artificially generated recognition sites have the shapes, sizes and functionalities that are complementary to the target analyte. Then the whole analyte recognizes the spatial area arranged, remaining "captured" in the polymer. This process can be developed for one single functional group or for a mixture of functional groups. These artificially generated binding sites, is a very powerful strategy providing the recognition sites a preference for the analyte, in detriment to other closely related structures. [Huang, 2004]. Although the affinity bioreceptor-analyte is less than if real biomolecule receptors were used, it has shown great mechanical stability over time, regarding other immobilization techniques.

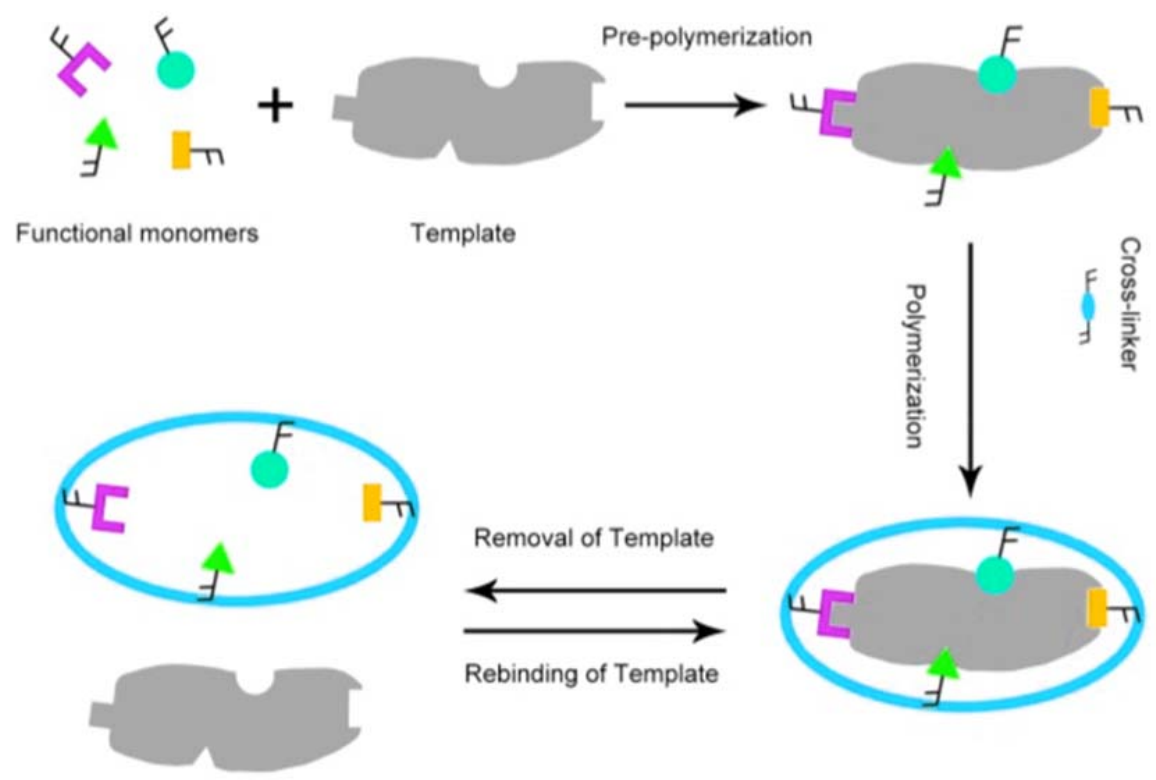

Figure 23: MIP fabrication scheme. [Vasapollo, 2011]. 
For the preparation of MIPs, the bioreceptor is attached to a template through covalent or non-covalent binding of a crosslinking monomer. Then it is performed the polymerization of the material and the bioreceptor is removed, leaving a replica of the binding areas of the bioreceptor, on the polymer.

\subsubsection{Polymers based surfaces modification}

As aforementioned in this work, it may be that biosensors transducers are composed by polymeric material due to their optical properties, and also to the cost efficacy in the fabrication process. [Sanza, 2011]. These materials can be polytetrafluor ethylene (PTFE), polypyrole (PPY), polydimethylsiloxane (PDMS), polymethylmetathacrylate (PMMA) or SU-8 microresist. [Goddard, 2007], [Laguna, 2014].

For the biofunctionalization of this type of polymeric materials, sometimes some pre-treatment procedure must be accomplished. Normally these procedures are based on chemical treatment, or exposures to ionized gas and UV radiation. The most used techniques are normally the chemical treatment or wet etching due to its great simplicity.

For example in SU-8 based biosensors, a wet etching of sulfuric acid $\left(\mathrm{H}_{2} \mathrm{SO}_{4}\right)$ is used for the activation of the epoxy groups remaining in the surface of the resist. (Figure 24) [Tao, 2008], [Joshi, 2007].

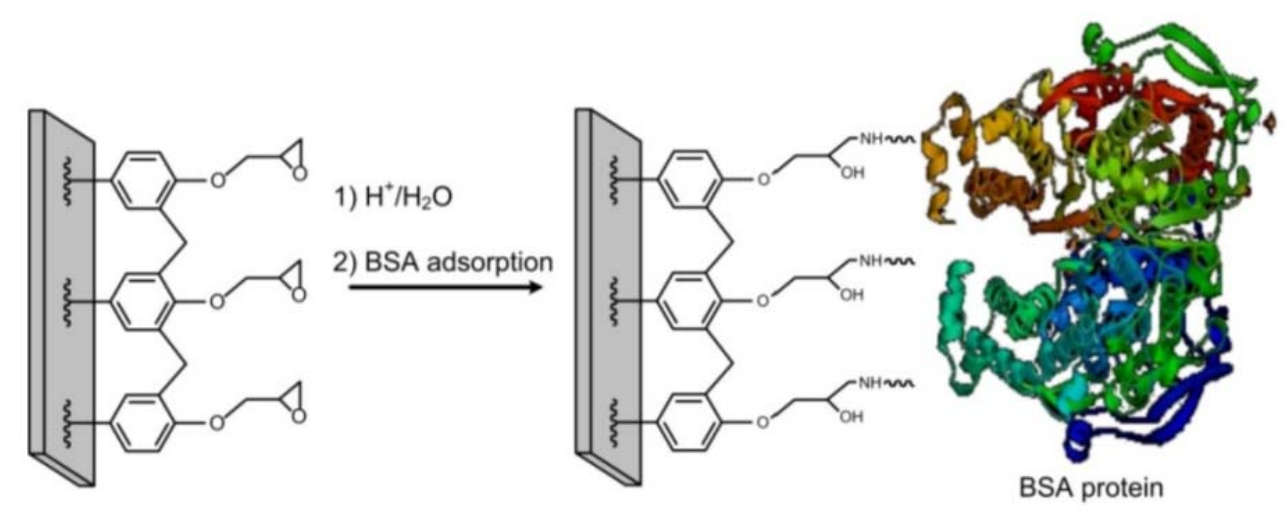

Figure 24: Activation of epoxy groups in SU-8 resist by sulfuric acid. [Sanza, 2015]. 
Other example is the PPMA surfaces being hydroxylated by sodium hydroxide $(\mathrm{NaOH})$ and $\mathrm{H}_{2} \mathrm{SO}_{4}$, for the generation of carboxylic acid groups.

However, these methods based on chemical etching are not isotropic, and provokes inhomogeneities along the surfaces; in addition they generate great amounts of chemical wastes.

Plasma obtained from ionized gases such as $\mathrm{Ar}, \mathrm{N}_{2}$ or $\mathrm{O}_{2}$, is a considerable option for the same purpose, since they are rather clean and prevent the material degradation or roughness regarding the chemical etchings. A different gas is used for the material to be treated according to the nature of the functional groups more accurate for the specific bioapplication being tested.

\subsubsection{Surface modification strategies}

This procedure is applied to perform a covalent bond of the biomolecules over the sensing transducers based on inorganic materials (silicon oxide, silicon nitride, silicon, etc). The covalent binding of the biomolecules permits a very stable biofunctionalization, able to undergo regeneration cycles in which analyte is separated from bioreceptor meanwhile bioreceptor remains attached to the surface for a next detection cycle. Besides, by controlling the surface chemistry, the distances of the functional groups to the surfaces can be checked for a better molecule orientation and/or antifouling purposes. [Trilling, 2013].

Probably, the golden star of the surface modifications strategies in silicon oxide based surfaces, is the silane-based chemistry. (Figure 25.A). (Especially for integrated optical biosensors). It employs organofunctional alkoxysilanes, that reacting with hydroxyl moieties present on the surface, creates self-assembled monolayers (SAMs). Silanes are formed by the alcoxy groups, (binding sites to inorganic surface) joined to the silicon (Si), and an organic fraction (also joined to the silicon) composed by the alkilic chain and the functional group. The alkilic chain acts a spacer between $\mathrm{Si}$ and the functional group, which binds covalently to 
the bioreceptors functional groups, creating an estable biofilm of bioreceptors fo the target analyte binding and detection.

Before silanization (process of surface functional groups modification, with organosilanes), silicon oxide based interferometers, must be turned in to hydrophilic surfaces, by being properly cleaned with different solvent, and activated by plasma oxygen or piranha solution, leaving $\mathrm{OH}$ groups available on the surface for the reaction with the alcoxy groups of the silane. The silane exposition to those activated surfaces provokes the growing of alkylsiloxane monolayers, such as trichloro-, trimethoxy- or triethoxysilanes, onto the solid substrate. In fact most of the times organic monolayers formation on silicon oxide, involves the reaction between silanol groups $(\mathrm{Si}-\mathrm{OH})$ present on the oxidized silicon surface.

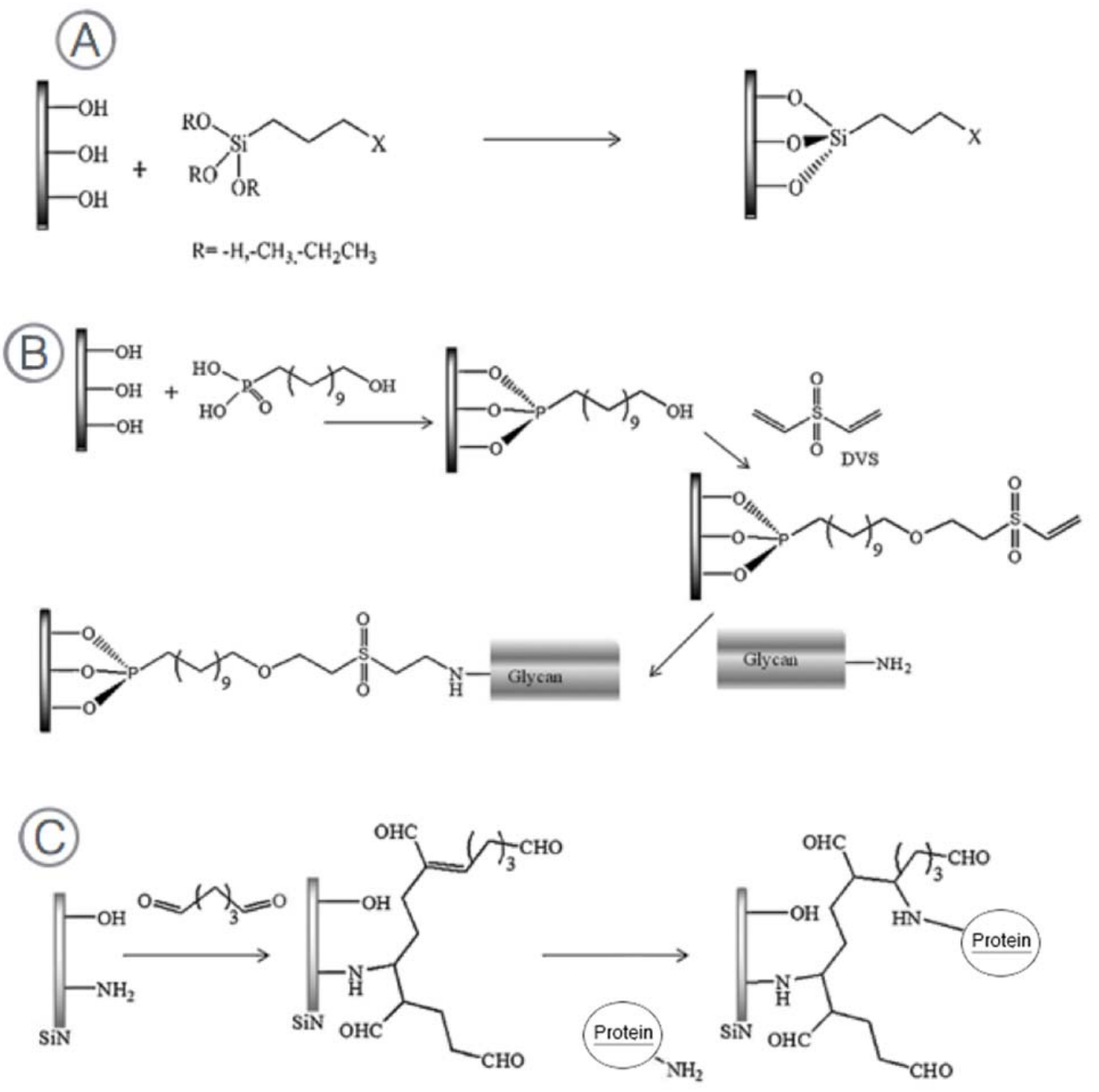

Figure 25: Surface modification techniques for silicon oxides surfaces. A Silanization. B. Phosphate chemistry. C. Glutaraldehyde linker path. [Bañuls, 2013]. 
There are several types of silanes commercially available, classified as a function of their functional groups or their inorganic binding sites: Aminopropyltrimetoxysilane (APTMS), Glicidopropyl-trimetoxysilane (GPTMS), Glicidopropyltrietoxysilane (GPTES), Aminopropyl-trietoxysilane (APTES), Aminopropyldietilmetoxisilane (APDMS).

APTMS and APTES are the most commonly used linkers for surface modifications in biofunctionalization processes. They form very stable bond to the surface, nonetheless, the silanes with more than one alcoxy group, tends to polymerize, due to their sensitiveness to be hydrolyzed. Now a days the tendency is to use silanes with one single binding site to the inorganic material. [Kim, 2009].

Phosphate chemistry is another surface modification strategy used for the formation of SAMs over silicon-based surfaces. For this purpose there are used hydroxyl-ended organophosphonated and a divinylsulphone (DVS) as a linker to bind the aminated glicanes (Figure 25.B) [Shang, 2012].

Bañuls research group has worked on the derivative silicon nitride surfaces, with glutaraldehyde through surface $\mathrm{N}-\mathrm{H}$ bonds after removing the silicon oxide native layer (Figure 25.C). This allows the selective attachment of amine groups against silicon oxide [Bañuls, 2013].

There are many others upcoming techniques for surface modification strategies purposes such as the alkene-based self-assembled monolayers grafted on oxidized platinum surfaces. [Alonso, 2015].

Han Zuilhof and collaborators are working on new surface chemistry modification by using hydrosilanes. Despite the simplicity of some of the approaches aforementioned, often they are time-consuming, and even irregular monolayers are created, if not undesirable polysiloxane networks are formed. This new hydrosilanes chemistry is supported by the catalyst tris (pentafluorophenyl) borane $\left[\mathrm{B}\left(\mathrm{C}_{6} \mathrm{~F}_{5}\right)_{3}\right]$, which acts as a promoter of $\mathrm{Si}-\mathrm{H}$ bond activation, enabling the 
stable and not hydrolizable monolayer formation in less than 5 minutes. [Escorihuela, 2017].

Once the surface has been optimally modified, obtaining the adequate functional groups, the surface is ready to be biofunctionalized with the specific bioreceptor. Nevertheless in some cases for this to occur, the functional groups still need to be activated by some mediators or cross-linkers that vary according to the functional group to be activated. (Figure 26). Disuccinimidyl carbonate (DSC), Carbonyldiimidazol (CDI), carbodiimide (EDC), N-Hydroxisuccinimide (NHS) and the popular avidin or streptavidin/biotin conjugation are some of them.

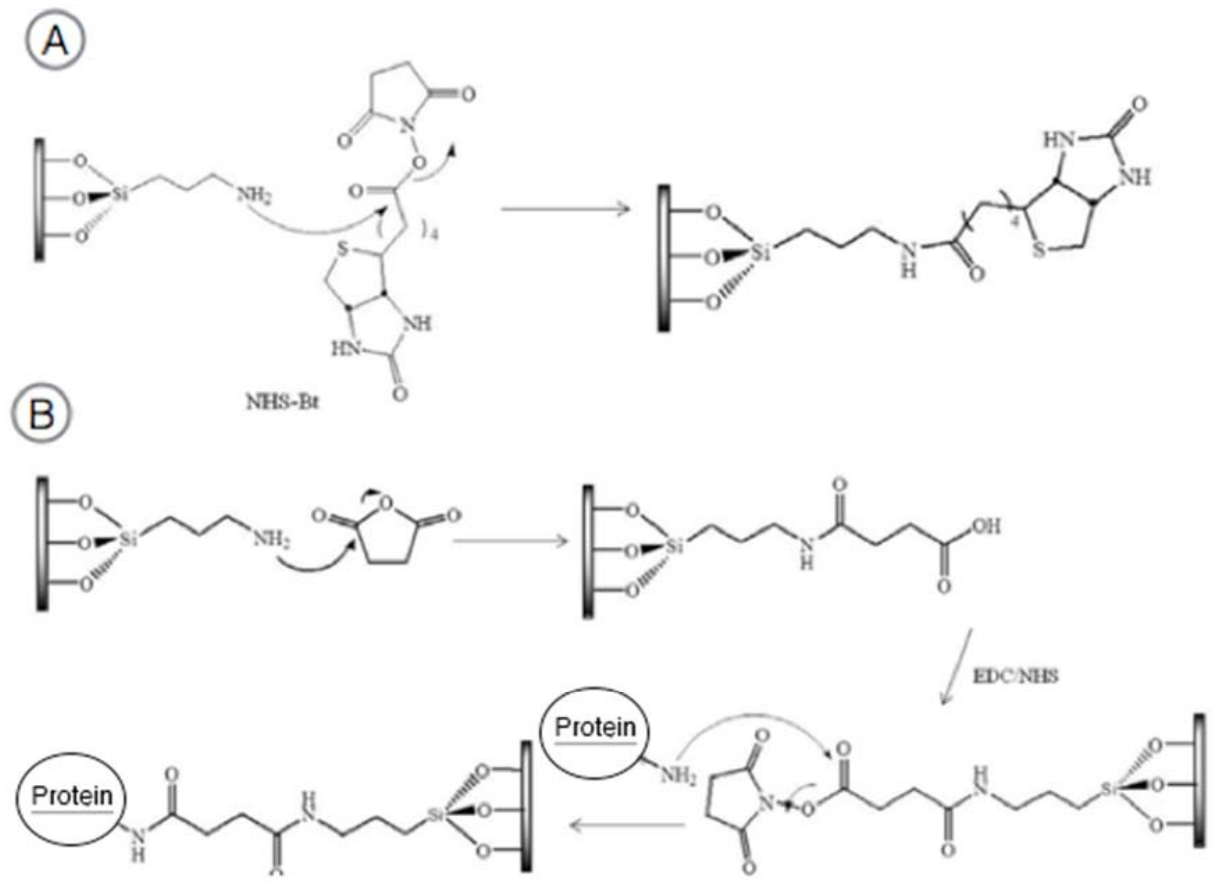

(C)

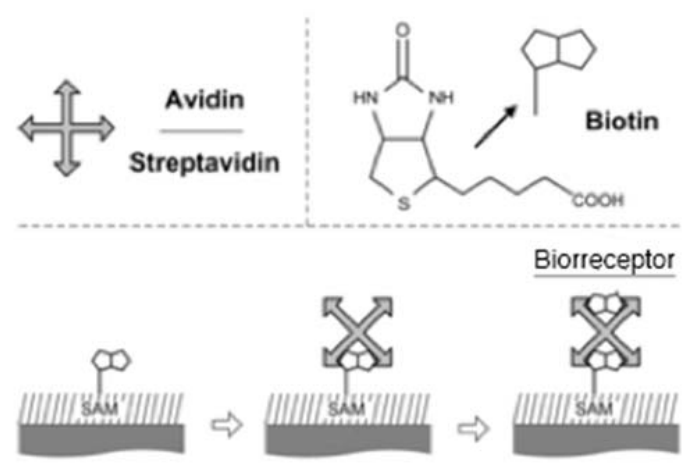

Figure 26: Activation of modified surfaces. A NHS linker. B. ECD linker. C. Avidin/biotin coupling strategy. [Bañuls, 2013]. 
In order to gain further knowledge about some of the protocols and different combinations of the development of the biofunctionalization sensing surfaces it is extremely recommended the review of Bañuls and collaborators on chemical surface modifications for the development of silicon-based label-free integrated optical (IO) biosensors. [Bañuls, 2013].

\subsection{Figures of merit of a biosensor}

There exist different magnitudes commonly studied in order to assess and compare the performance of different biosensors.

\subsubsection{Sensitivity}

Sensitivity is a physical magnitude of the sensing transducer, defined as the transducing signal variation, as a function of the concentration of the target analyte. In refractive optical biosensors, for biochemical detection, the sensitivity can be calculated studying the shift of the resonant mode (Aresonance) of the optical response, normally measured in wavelength $(\mathrm{nm})$ or in wavenumber $\left(\mathrm{cm}^{-1}\right)$, as function of the variation of the Refractive Index $(\Delta n)$ of the media, which is the same as Refractive Index Units (RIU). (Equation 4). This is conventionally known as the bulk sensitivity of a biosensor. (Figure 27.A).

$$
\text { Bulk sensitivity }=\frac{\Delta r e s o n a n c e}{\Delta n}
$$

The bulk sensitivity is a good figure of merit to compare the performance of different optical biosensors independently of the molecules to be detected.

But the real sensitivity of a biosensor; biosensitivity (equation 5), is determined considering the slope of the linear range of the biosensing response. The slope is calculated studying the shift of the resonance mode as a function of the variation of the concentration of the target analyte. The linear range goes from the first concentration point measured, to the saturation point, in which the addiction of 
analyte concentration barely produces a change in the resonance mode. (Figure 27. B).

$$
\text { Biosensitivity }=\frac{\Delta \text { resonance }}{\Delta \text { concentration of analyte }} \quad \text { (Equation 5) }
$$
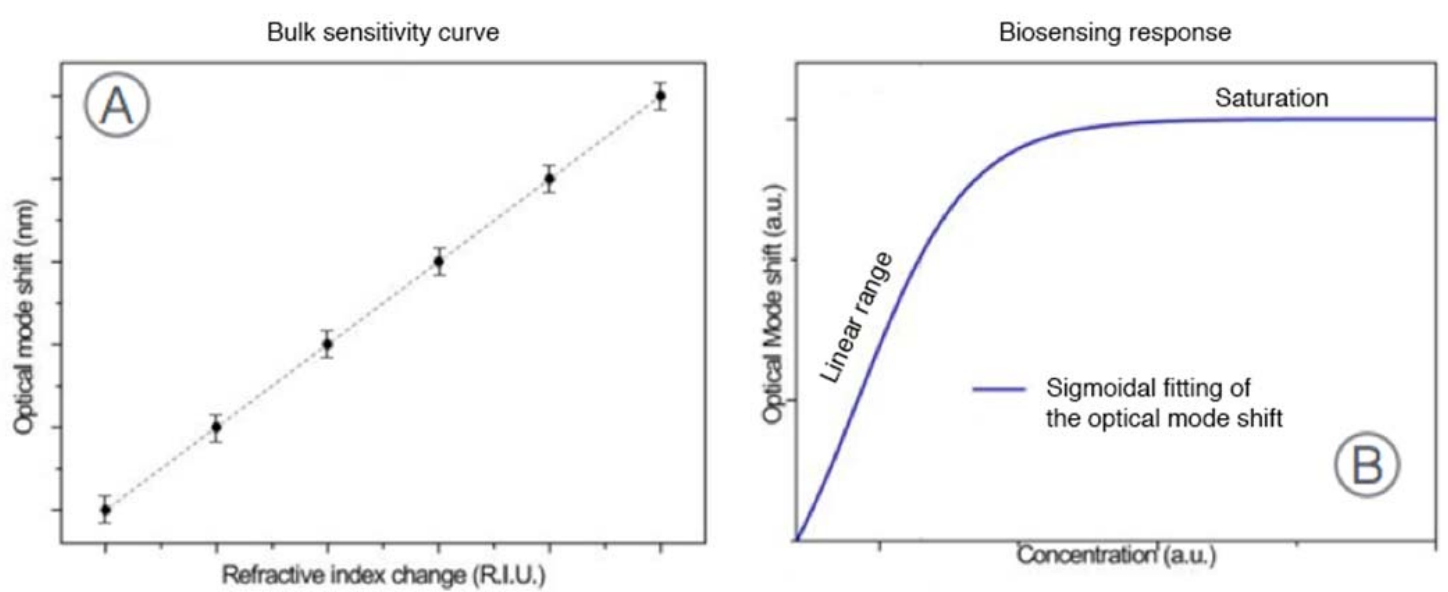

Figure 27: A. Regression line for bulk sensitivity calculation in a bulk sensing response.

B. Sigmoidal fitting of a biosensing response, for calculation of biosensitivity from the slope of the linear range.

Even though, bulk sensitivity and biosensitivity are experimental parameters. In order to predict the sensitivity of a biosensor when detecting biomolecules, it was defined the Sensing Quality Factor (SQF) [Lavin, 2013]. It is a theoretical parameter that takes into account the variation of the resonance mode as a function of the biolayer thickness. It allows comparing the sensing response of two different biosensors for the same biological detection.

$$
S Q F=\frac{\Delta \text { resonance }}{\Delta \text { thickness biolayer }}
$$




\subsubsection{Limit of Detection (LoD)}

Nevertheless, the sensitivity only bears the maximum spectral shift, obviating other parameters involved in the biosensors operationally, as for example the resolution of the system, or the uncertainty of the measurement and other noise sources. For this reason it is studied the limit of detection which is defined as the minimum concentration of analyte that the biosensor is able to detect [IUPAC]. This is a much powerful parameter to compare biosensors since it provides information about the entire process taking place in the biosensing response [Hernández, 2015], [Santamaria 2017].

It can be expressed as:

$$
L o D=\frac{U}{\text { sensitivity }}
$$

Where $U$ is the denotation of the expanded uncertainty, which is the uncertainty (u) multiplied by a factor of coverage which is normally three for biological systems following the Union of Pure and Applied Chemistry recommendation (UIPAC):

$$
U=3 u
$$

(Equation 8)

The Guide to the Expressions of Uncertainty in Measurements (GUM) indicates that the uncertainty $(u)$ should be calculated as:

$$
u^{2}=\frac{R^{2}}{12}+\frac{s^{2}}{n}
$$

Where $R$ is the resolution of the used measuring system, and $s$ is the standard error drawn from repeated measurements for a more robust statistically results. $n$ is the number of repeated measurements. 


\subsubsection{FWHM and Q-factor}

According to equation 7 it can be inferred that final LoD does not only depends on the sensitivity of the sensor, but also the uncertainty is a conditioning value. In theoretical models, it is desirable to determine a parameter, able to provide information about the uncertainty of the biosensor in order compare different designs performances and do not rely only on the SQF. For this, it is studied the Full Width at Half Maximum (FWHM), this is, the maximum width of the resonant mode at the half of its intensity. A low value of FWHM implies an accurate determination of the position of the resonant mode. This improves the LoD of the biosensor, because the uncertainty on the position of the resonance is decreased.

Q-factor is a parameter that measures the effect of the value of the FWHM on the optical biosensors performance. For optical signals depending on the wavelength $(\lambda)$ it can be calculated as:

$$
Q-\text { factor }=\frac{\lambda \text { position }}{\text { FWHM }}
$$




\section{OBJECTIVES OF THIS THESYS AND MOTIVATION OF THE USE OF R-NPS}

The main goal of this doctoral thesis is the development of a new label-free optical biosensor of high sensitivity based on a novel transducer of resonant nanopillars arrays, which is capable of detecting biological species and optical properties of liquids through optical interrogation. The characteristics of the biosensor will be: high performance, multiplexed capability, and good sensitivity and cost-effectiveness.

\subsection{Objectives}

The specific objectives pursued in this scientific work are summarized as:

- Development and fabrication of an optical transducer based on R-NPs. The fabrication should be directed towards a mass scale fabrication, compatible to standard micro-nano fabrication processes used in the microelectronic industry.

- Design and assembly of an optical read-out system based on optical vertical interrogation which can be used for R-NPs measurement in dry or in fluidic conditions and susceptible to be coupled to fluidic systems for real-time experiments.

- Design and demonstration of the optimal fabrication parameters of the R-NPs for the optimization of the optical response and for their distribution into independent arrays.

- Assessment of the performance of the R-NPs as biochemical sensors, through bulk sensing experiments based on the detection, in static and in continuous flow, of several fluids with different refractive index. 
- Biofunctionalization of the R-NPs to be used as label-free biological sensors. Development of different bioreceptor immobilization protocols for an effective analyte detection.

\subsection{Why R-NPs as biological sensors?}

The development of resonant nanopillars arrays to be used as label-free optical biosensors is encouraged for reasons of various kinds:

\section{Photonic properties:}

o R-NPs are based on Bragg reflectors and a central cavity that, under optical interrogation, provoke the formation of a significant resonant mode easy to interpret along the experiments.

o The grouping of the resonant nanopillars into resonant nanopillars networks promotes the light confinement among the pillars, enhancing therefore the sensitivity of the transducer.

2. Robustness: The distribution in periodic arrangements provides redundancy in the optical response. This means that, in the event that some of the nano-pillars in the network are destroyed, the optical response will not significantly be changed because the rest of the R-NPs still will produce the signal. This, together with the hardiness of the materials with which they are made of: $\mathrm{Si}_{3} \mathrm{~N}_{4}$ and $\mathrm{SiO}_{2}$, provides R-NPs arrays with a great robustness.

3. Optical properties: The advantage of the R-NPs from the optical point of view, is that they can be vertically interrogated easily, avoiding complexity of light coupling as it occurs in planar-based biosensor to read-out the information coming from a waveguide, where inverted taper o gratings are normally used to couple the light into a fiber optic. 
4. Multiplexing capacity: R-NPs networks can be distributed into specific arrays or cells, which can be called BICELLs when they are properly biofunctionalized. BICELLs can be fabricated along the substrate, providing the sensor with multiplexing capacity for detecting several analyte at the same time. These types of sensors are called integrated biosensors.

5. Fluidic reasons: R-NPs can be used to take discrete measurements in dry conditions or for continuous flow measurements, in fluidic conditions. This is due to the fact that they are fabricated over a transparent quartz substrate and can be interrogated from the backside, with he R-NPs facing down in contact with the fluid.

6. Specific surface control: The fabrication of R-NPs arrays allows the user to control the specific surface to test. It can be increased or reduced by adjusting the R-NPs fabrication parameters.

7. Biochemical reasons: Materials forming R-NPs $\mathrm{Si}_{3} \mathrm{~N}_{4}$ and $\mathrm{SiO}_{2}$ are susceptible to be biofunctionalized with biological receptor, providing the capacity to R-NPs to operate as biological sensors.

8. Cost effectiveness: R-NPs chips have a production price in laboratory conditions of around forty euros, which could be potentially decreased if produced at mass scale.

Next table summarizes the exposed reasons for the use of R-NPs as biosensors, highlighting the main advantages provided. 
Table 1: Motivation of the use of R-NPs as chemical and biological sensors.

\begin{tabular}{|l|l|}
\hline Reasons & Advantages \\
\hline Photonic properties & $\begin{array}{l}\text { Resonant mode formation and light } \\
\text { confinement in R-NPs networks }\end{array}$ \\
\hline Robustness & Redundancy of the signal \\
\hline Optical properties & Easily vertically interrogated \\
\hline Multiplexing & Several analyte detection \\
\hline Fluidics & Good wettability \\
\hline Specific surface control & Control of the surface area \\
\hline Biochemical reasons & Biomolecules attachment \\
\hline Cost-effectiveness & Competitive production cost \\
\hline
\end{tabular}




\section{RESONANT NANOPILLARS ARRAYS}

The nanotechnology together with the use of different materials with singular optical properties, have enhanced the use of novel micro- and nanophotonic structures for different applications. This chapter is a description of the advantages of using such nanostructures. First it is explained the effect of using nanopillars based optical interferometers. Then, there are explained the physical principles, that motivate the use of Bragg reflectors and a central cavity in such nanopillars structures, conforming the Resonant Nanopillars (R-NPs).

\subsection{Nanopillars for sensing}

Nanostructured pillar based biosensors have been largely reported in the literature, such as silicon nanopillars, [Kugel, 2014] or silicon oxide nanopillars [Trifonov, 2005]. Also nanopillars composed by different materials like silicon and silicon oxide have been proved [Choudhury, 2014]. In addition means of SU-8 nanopillars arrays (BICELLs), have been objet of studio in the recent years [Casquel, 2011], [Holgado et al., 2010]. Moreover, 2D array of SU-8 nanopillars fabricated on an optically thick aluminum layer have proven to be effective label-free optical biosensors. [Canalejas, 2016]. Periodic nanopillars for sensing are represented in figure 28.

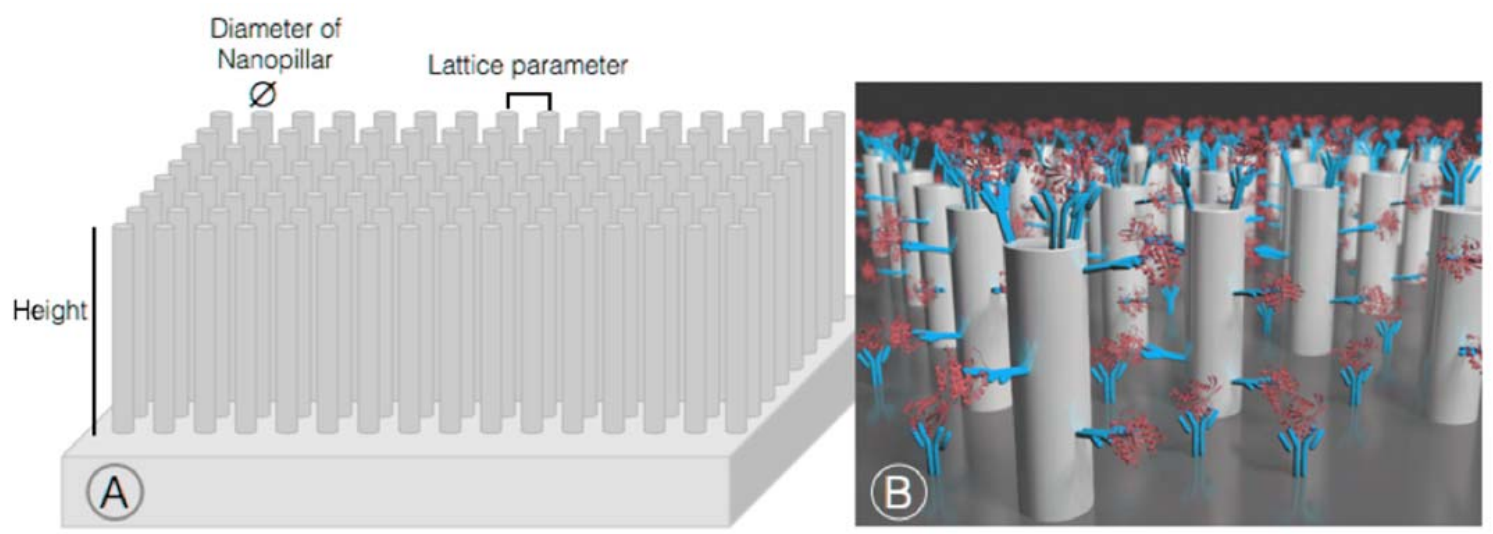

Figure 28: A. Periodoc nanopillars arrangement. B. Nanopillars array with antobody immobilized and analyte recognition. [Getty images]. 
One of the advantages of the use of nanopillars is the increased sensing area regarding planar Fabry-Perot interferometers. (Figure 29). In this case of study, nanopillars distribution is based on triangle networks of an area $=(\sqrt{3} / 4) a^{2}$. For the calculation of the unitary cell area (minimum periodic cell), it is considered the lattice parameter $a$ (the distance among pillars, see figure 28), and the surface fraction of the nanopillars included in the triangular area, which is in total one pillar. Then, given a radio $r$ and a height of the pillars $h$, it can be easily calculated the area of one single unitary sensing cell.

(A) Bulk sensing cell

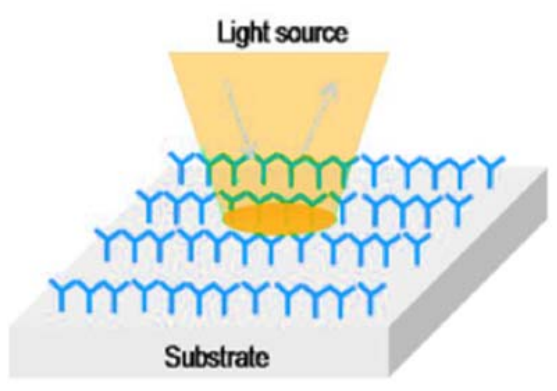

(B) Pillars sensing cell

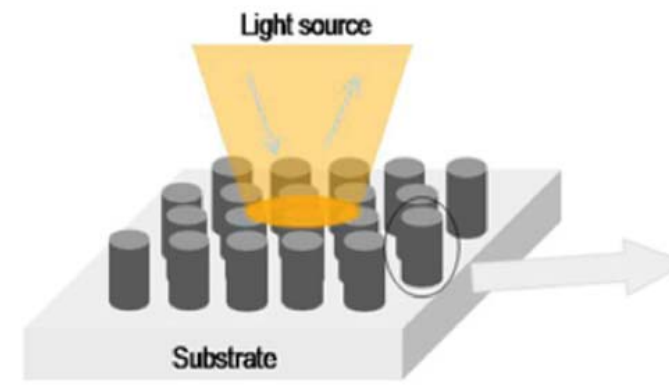

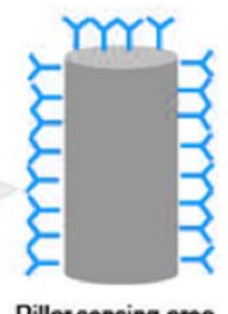

Pillar sensing arca

Figure 29: A. Fabry-Perot sensing cell. B. Nanopillars array sensing cell. [Casquel, 2012].

$$
\text { Area of unitary cell }=\left(\frac{\sqrt{3}}{4} * a^{2}\right)+h * \pi * r^{2}
$$

(Equation 11)

Then, the increased factor per unitary area is:

$$
\text { Increase factor }=\frac{\left(\frac{\sqrt{3}}{4} * a^{2}\right)+h * \pi * r^{2}}{\left(\frac{\sqrt{3}}{4} * a^{2}\right)}
$$

Other benefit of the use of nanopillars periodic arrays is their capacity for light confinement, enhancing the sensitivity of the biosensor. The effect is similar to the effect that is taking place in waveguides, but in this case the difference of RI is between the nanopillar and the surrounding material. This can also be the biomolecule attached to the nanopillars surface. The light confinement is higher when the lattice parameter is lower. In figure 30 is shown the influence of the 
lattice parameter on the light confinement. For smaller lattices or periods it is obtained highest intensity in the low index region. In addition a diminution of the nanopillars diameter also increases the evanescent field in the surface, but this parameter is strictly limited by the fabrication resolution and constrains.
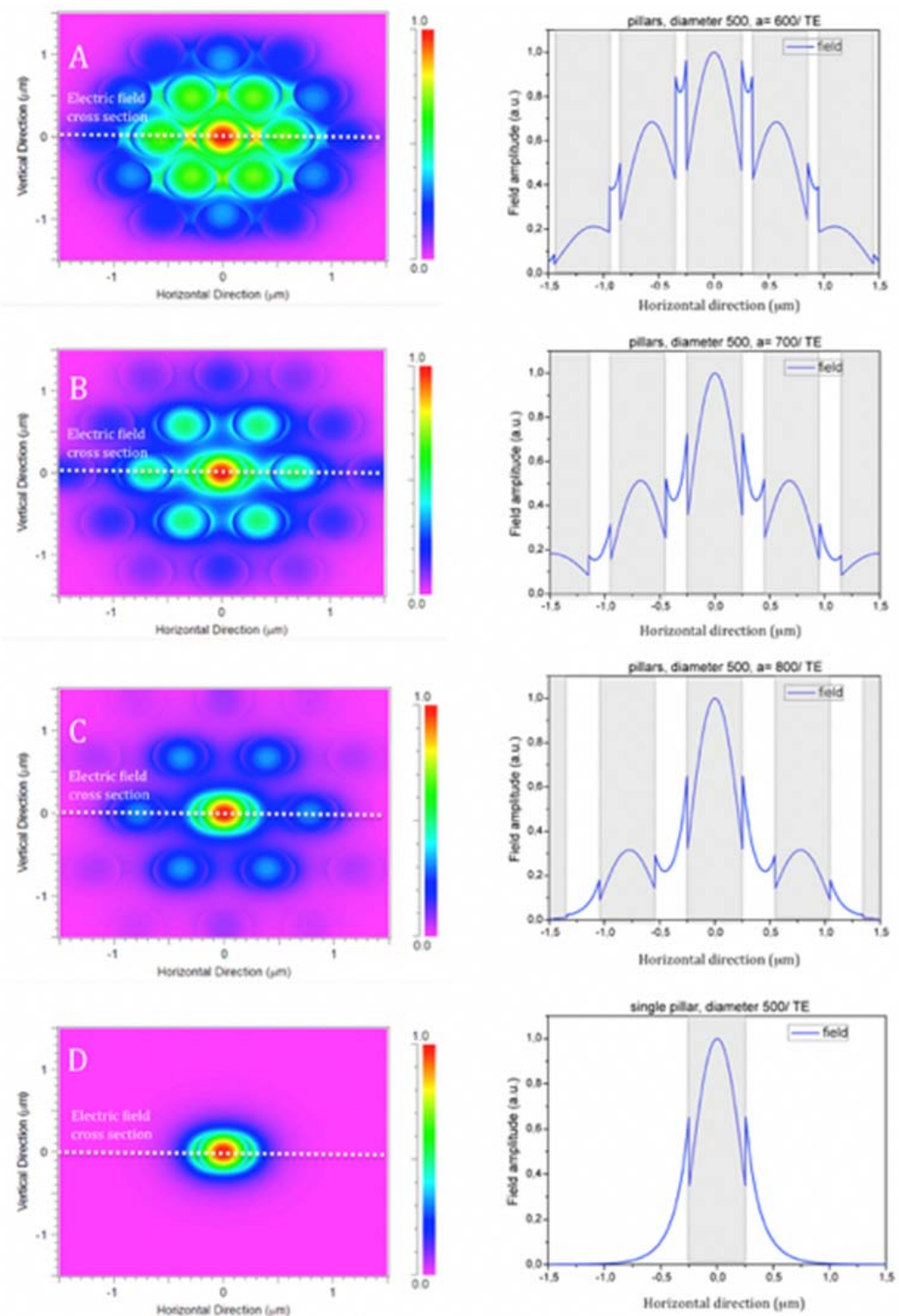

Figure 30: Light confinement in different nanopillars lattice parameter. From lowest lattice parameter (A), to a single nanopillars (D). [Casquel, 2012].

The lattice parameter is also restricted by fabrication parameters and by physical instabilities of such small and close nanopillars, falling ones upon the others. 
In addition when designing nanopillars lattice parameter, it must be studied the wettability properties depending on the hydrophobicity of the materials and distances among the nanopillars. Very small lattice parameter can prevent the liquid infiltration in the nanopillars lattice, hampering their sensing capacity. (Figure 31).

(A) Hydrophobic Walls

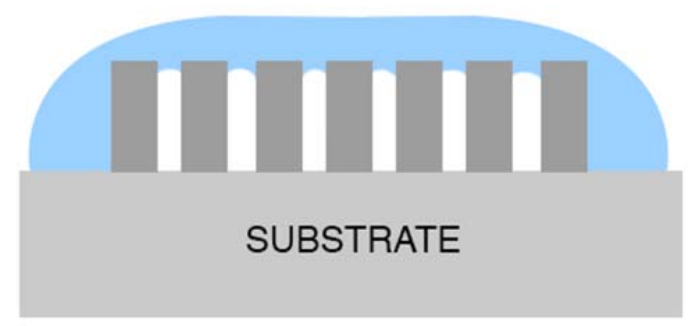

(B) Hydrophilic Walls

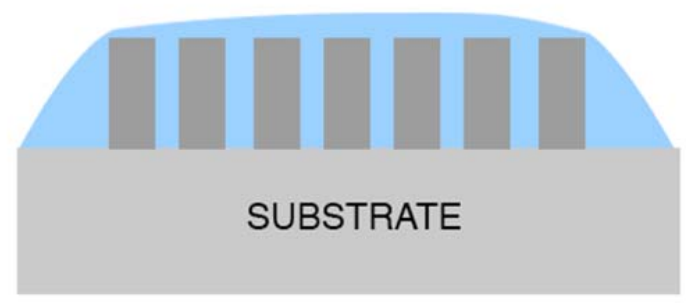

Figure 31: A. Hydrophobic nanopillars. B. Hidrophilic nanopillars.

\subsection{Photonic properties of the Resonant Nanopillars}

The studied sensors are label-free optical biosensor based on resonant nanopillars (R-NPs). Refractive Index changes surrounding R-NPs, can be observed through the shift of the optical resonant mode formed when the R-NPs are optically interrogated.

In this thesis it is proposed a singular transducer, based on nanopillars for optical label-free detection. Certain materials form the nanopillars, with different refractive index, (RI) in a specific arrangement, which provide them with unique optical properties. Nanopillars consist on several pairs of $\mathrm{SiO}_{2} / \mathrm{Si}_{3} \mathrm{~N}_{4}$ Bragg reflectors and a central cavity of $\mathrm{SiO}_{2}$. [Hernandez, 2015]. For this reason they are called Resonant Nanopillars (R-NPs). They are distributed into in a quartz substrate, forming arrays that can be independently, vertically and optically interrogated for the detection process, thus they can be included in the category of BICELLs in label-free optical biosensors. 
To be able to expose the optical effect that takes place when the R-NPs are vertically interrogated and thus understand the optical properties that endorse the advantages of the use of this novel biosensor, it is essential to bear in mind the laws of reflection and refraction of the light, as well as the concept of refractive index (RI, also denoted as $n$ ) of the different media.

The refractive index of a media $(n)$, express the relation between the speed of the light in the vacuum $(c)$ and the speed of the light in that media $(v)$. Thus the higher it is the speed of the light in one media; the lower will be the $n$ of that media, in vacuum $n$ is equal to one.

$$
n=\frac{c}{v}
$$

When an electromagnetic field, (such as light) passes from one media (n1) to another media with a different RI $(n 2)$, at a certain angle $(\boldsymbol{\theta} i)$, it will be partly reflected into the first media within an angle $(\boldsymbol{\theta} r)$ equal to $\boldsymbol{\theta} i$ (Law of Reflection), and partly will be refracted into the second media within a different speed and direction caused by the change of RI of the media (n2). This change in light propagation is known as the refraction of the light and is commonly explained through the Law of Snell. [Snell Law], (Figure 32).

The Law of Snell claims that when the light reaches an interphase (with an angle of incidence $=\boldsymbol{\theta} i$ ) which separates two media with different RI, the product of the sine of the angle of incidence $\boldsymbol{\theta} i$ (with respect to the normal angle) and the refractive index of the media (n1), is equal to the product of the sine of the refractive angle $\boldsymbol{\theta} t$ and the RI of the second media (n2).

$$
n 1 * \sin \theta i=n 2 * \sin \theta t
$$




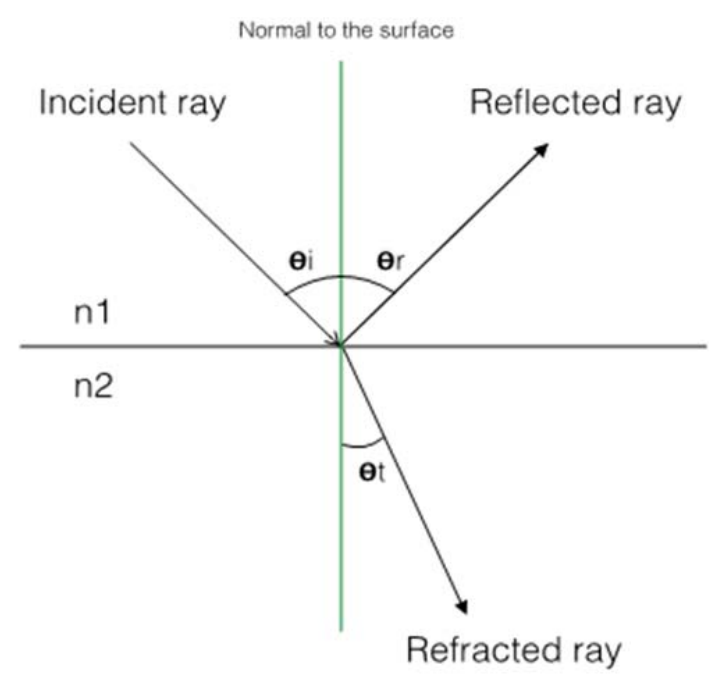

Figure 32: Explicative scheme of refraction and reflection laws.

This concept is important to understand the optical properties of a traditional Fabry-Perot interferometer (FP), which is the theoretical base on which the optical foundations of the R-NPs rely on.

FP interferometers are composed by two close plane mirrors (surfaces) and a planar microcavity between them. Part of the light is transmitted each time it reaches the second surface, producing multiple reflexions inside the planar microcavities, depending on the thickness and material of the cavity and also, on the wavelength and angle of incidence of light.

The plane mirrors can be disposed in several ways: metal thin-films (Figure 33.A), Distributed Bragg Reflectors (DBR) (Figure 33.B) or the thin film planar microcavities (Figure 33.C). 

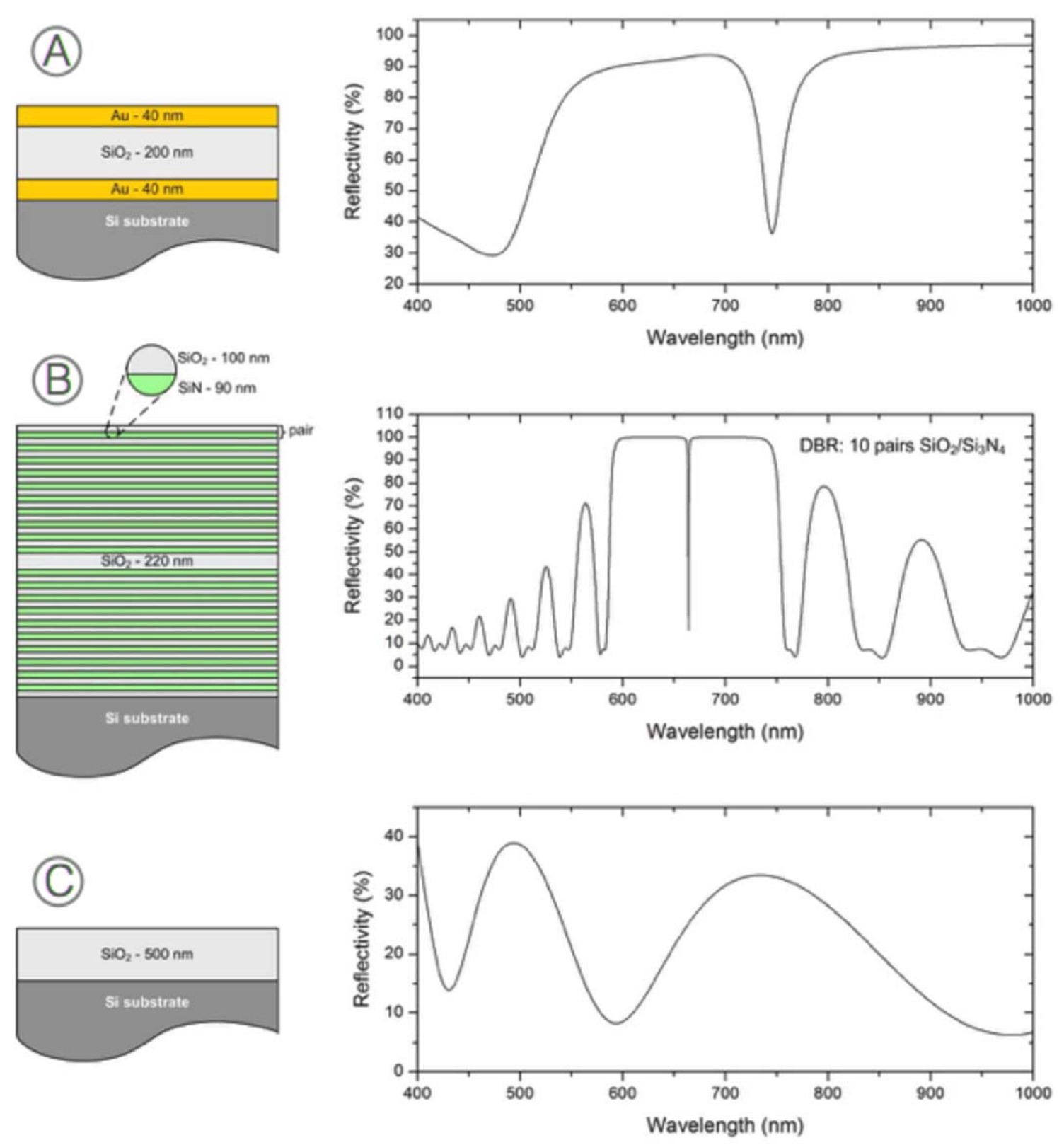

Figure 33: A. Metal thin film interferopmeters. B. DBR interferometer. C. Planar microcavity interferometer.

Metal thin-films experience high loss of intensity due to the absorption of the metallic materials. In contrast to this DBR are based on semiconductor materials and their reflectance can be controlled by the number of pair of layers, materials thickness and refractive index contrast. They are further studied in this thesis.

Lastly a thin film deposition over a reflectance material, compose the simplest FB interferometer. The cavity is the interface between materials, e.g. air/cavity or 
cavity/substrate. The GOPB has largely worked with this type of FP interferometers as label free optical biosensors based on polymers deposited over silicon or silicon oxide substrates.

\subsubsection{Distributed Bragg Reflectors (DBR) for R-NPs}

Each R-NP acts as a single 1D photonic crystal since they are composed by means of periodic thin films stacks on one dimension, made of dielectric materials with different RI. These periodic materials are $\mathrm{SiO}_{2}$ and $\mathrm{Si}_{3} \mathrm{~N}_{4}$ pairs acting as Distributed Bragg Reflectors (DBR) lying on a quartz substrate.

Distributed Bragg Reflectors or Bragg reflectors mirrors are composed by a multilayers stack alternating at least two dielectric adjacent materials with high (nh)-and low (nl)- refractive index consecutively. (Figure 34). When light strikes every layer boundary, part of it is reflected. If the RI contrast of the materials and their thicknesses are adequate, it can occur that, the accumulation of reflectivity coming from multiple reflections from periodic interphases, produces a constructive interference, of almost total light reflection, at a specific wavelength (Nightingale, 2008). This wavelength range is commonly named as photonic band gap (PBG). It means that, in that specific range, the light cannot go through the material and all the light is reflected. (Figure 34.B, photonic band gap). [Zhong, 2015].

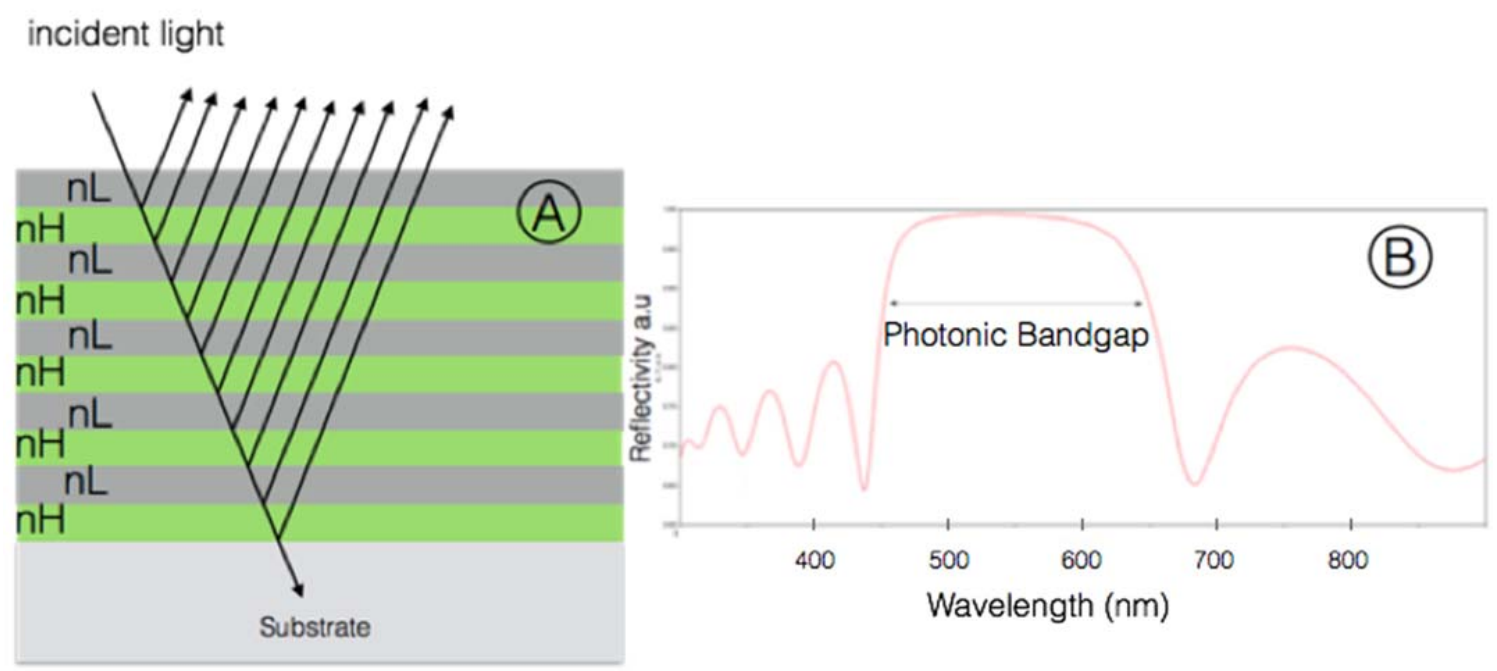

Figure 34: DBR. A. Scheme of composition and light direction. B. Photonic bandgap in the optical response. 
Adjusting the thicknesses of the layers $(t h 1, t h 2)$ and the RI contrast $(n 1, n 2)$ it can be controlled the position of the wavelength range where the photonic band gap is centered $\lambda / 2$ (equation 15).

$$
\operatorname{th} 1 * n 1+\operatorname{th} 2 * n 2=\frac{\lambda}{2}
$$

In the case of the R-NPs studied in this work, the material with lower RI (nL) is $\mathrm{SiO}_{2}(\mathrm{n}=1.4)$ and material with higher $\mathrm{RI}(\mathrm{nH})$ is $\mathrm{Si}_{3} \mathrm{~N}_{4}(\mathrm{n}=2)$. The higher it is RI contrast, the wider it is the band gap. And the more Bragg reflector pairs compose the multistack, the more defined is the band gap.

To provide R-NPs, sensing capabilities, Bragg reflectors multistack is designed with a photonic defect, just at the half of the height of the structure. This defect is a thin layer and can be translated in optical terms as resonant cavity, which has the function of acting as light strainer in the multistack. The resonant cavity is made in this case of $\mathrm{SiO}_{2}$ and optically, it can be noticed as a very narrow resonant mode localized just in the middle of the photonic band gap when the multistack is vertically interrogated with a white light source. The number of Bragg reflector pairs must be the same on each side of the central cavity. (Figure $35 \mathrm{~A}$ ).
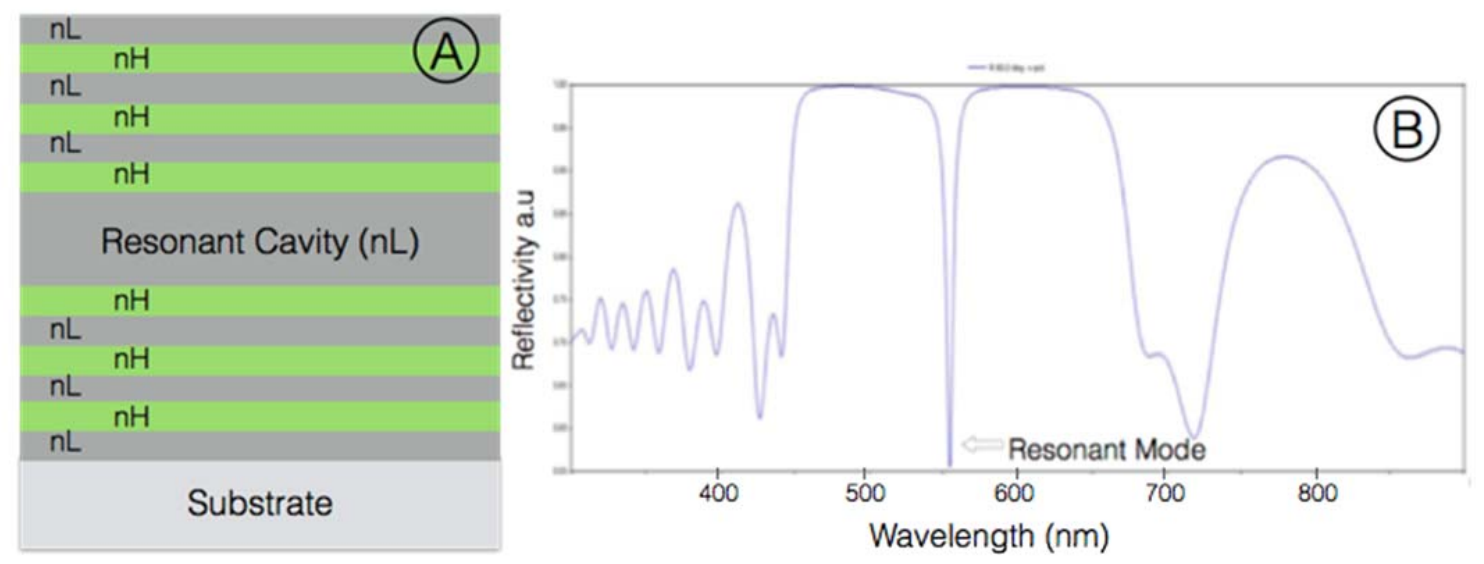

Figure 35: A. DBR with a central cavity. B. Resonance mode of the central cavity in the optical response. 
The formula for calculating the resonance mode position $(\lambda)$ in the band gap is as follows:

$$
\text { Th cavity }=\frac{\lambda}{2 n \text { cavity }}
$$

Where Th cavity is the thickness of the cavity, $n$ cavity is the Refractive Index of the cavity and $\boldsymbol{\lambda}$ is the position of the resonant mode.

When the R-NPs are fabricated from a multistack of thin films, the arrays of R-NPs form an effective nanostructured material in contact with the surrounding medium, and its effective refractive index changes with the refractive index of the medium. This means that the resonant mode, shifts along the wavelength range as a function of the refractive index of the medium surrounding the cavity, including biological material attached to it. (Figure 36). Thus, according to equation 16, the increase of the RI of the medium implies a shift of the resonance mode into a higher wavelength.
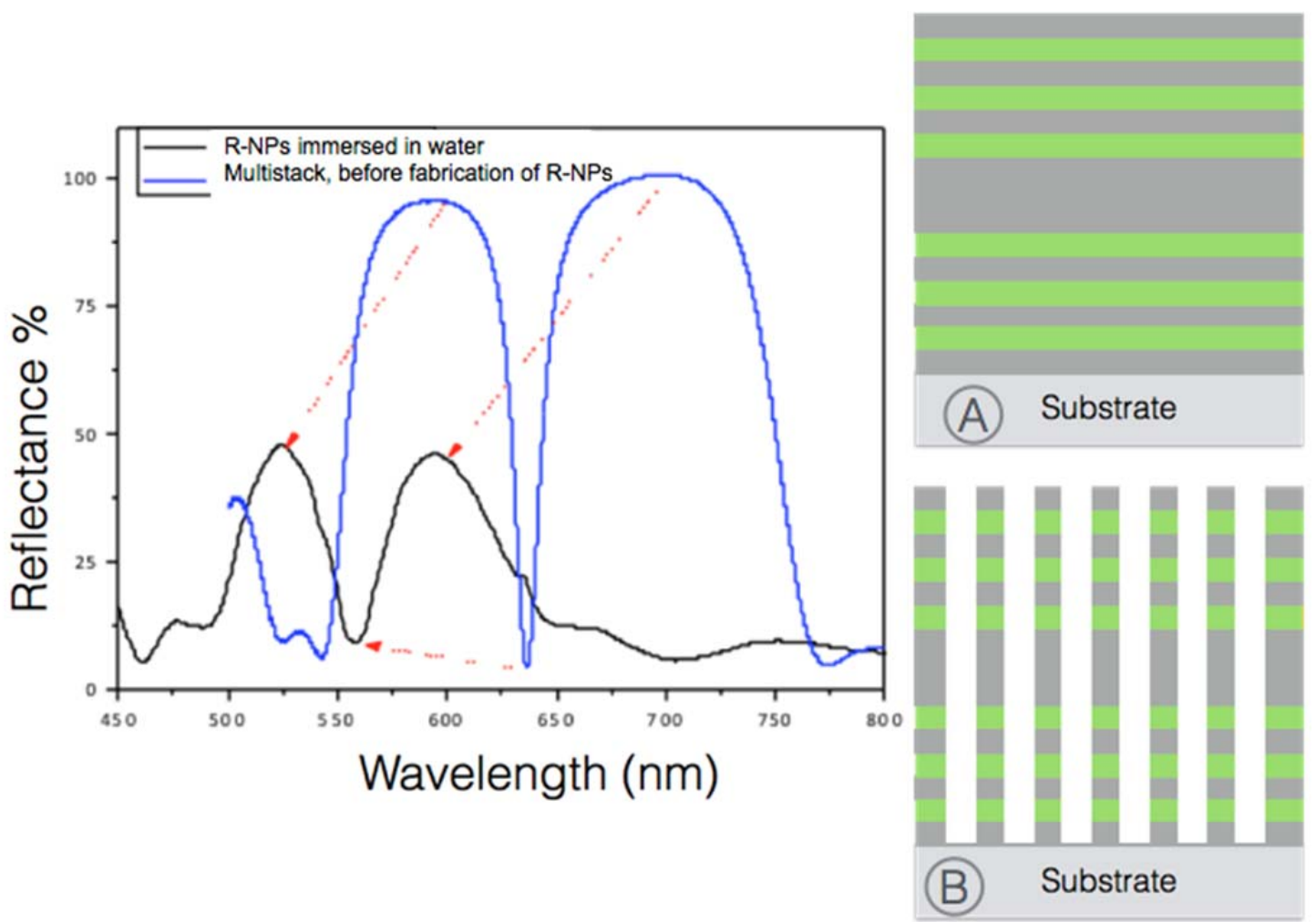

Figure 36: Optical response of the BDR with a resonant cavity, before (A) and after the fabrication of the R-NPs (B). 
The goal when designing R-NPs is to achieve a good sensing performance, for this purpose, it is necessary to have a well-designed band gap with a very narrow and defined resonance. During a biological recognition, the greater it is the shift of the resonant mode, the higher it is the sensitivity of the biosensor. (Figure 37).
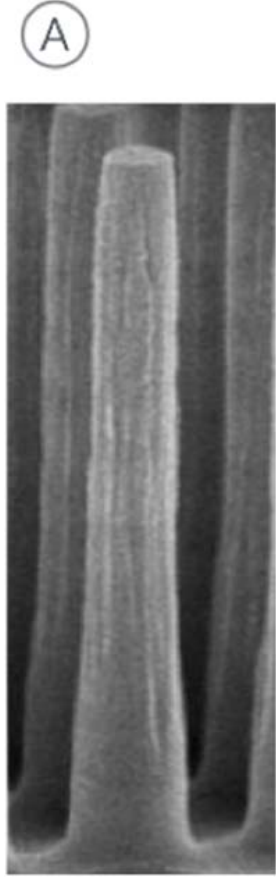

Reference

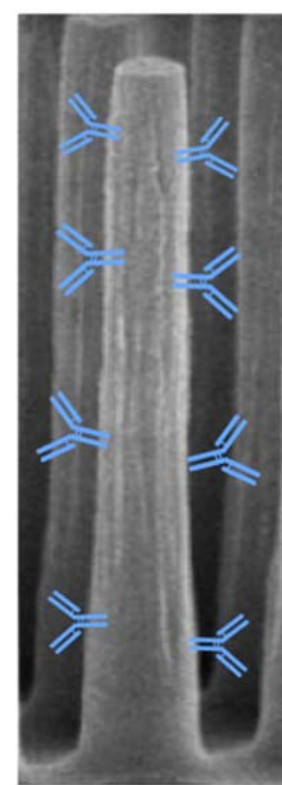

Biorreceptor

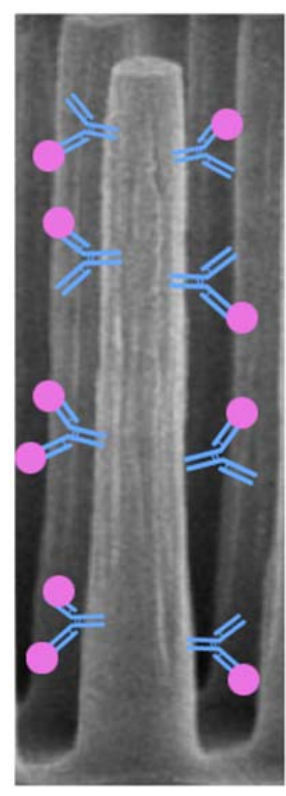

Recognition

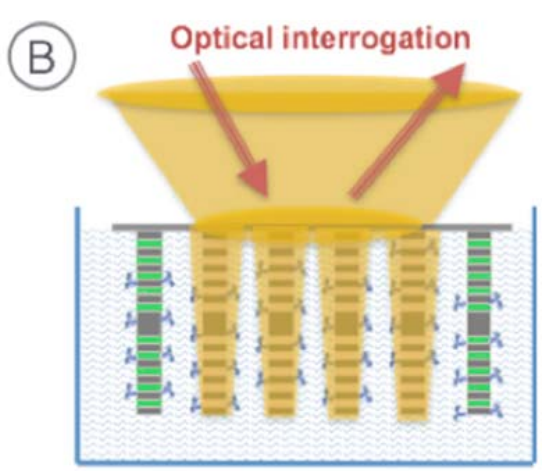

(C)

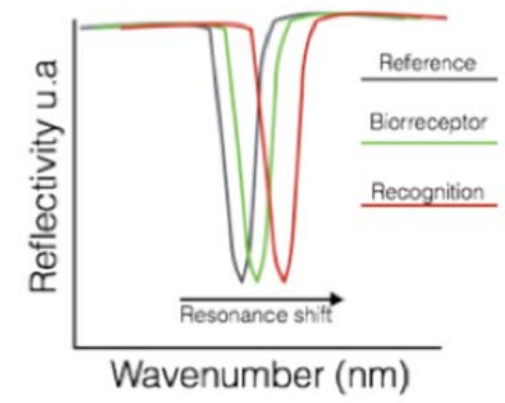

Figure 37: A. R-NPs during an immunoassay: Reference, R-NPs immobilized with bioreceptors and $R$-NPs recognizing the analyte. B. R-NPs with anitibodies being optically interrrogated. C. Resonant mode shift for the different steps of the immunoassay.

\subsubsection{Calculation of the effective RI for R-NPs design}

For the design of the R-NPs, firstly the materials of the DBR are chosen, and the resonance is fixed at a particular wavelength. Then, it must be calculated the effective refractive indexes $\left(n_{\text {eff }}\right)$ of the materials when the R-NPs are fabricated, considering a value for lattice parameter and diameter, and thus create a theoretical model that predicts the optical response of R-NPs. (Figure 38).

In former works the Group of Optics, Photonics and Biophotonics, from UPM, in order to be able to calculate the effective indexes, used as simulation system the 
program Rsoft, based on the Beam Propagation Method (BPM) and the Finite Difference Time Domain Method (FDTD), [Lavin, 2013], [Scarmozzino, 2000]. Even though those are good simulation programs, they are extremely time-consuming. For that reason in this case it was used the Maxwell-Garnett equation for effective index determination.

\section{A Periodic array of R-NPs \\ 3D photonic structure}

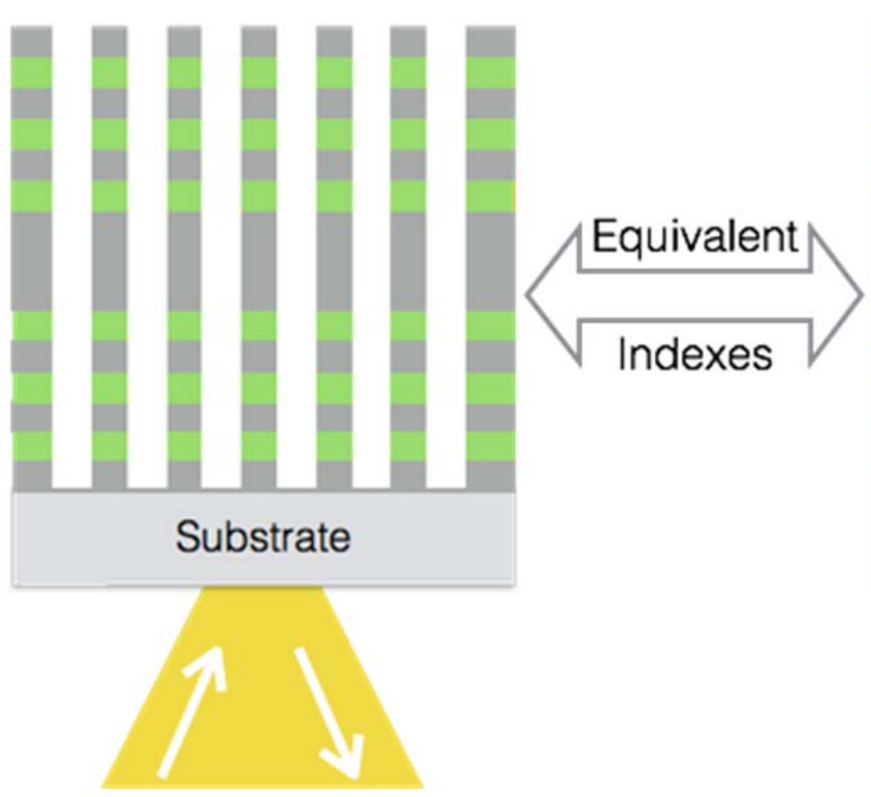

Light
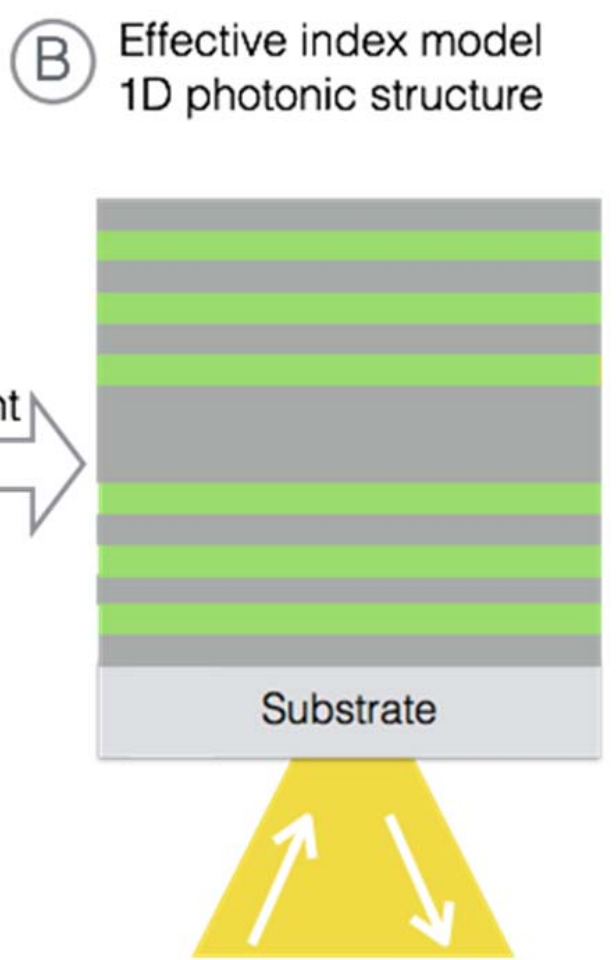

Light

Figure 38: A. R-NPs. B. Multilayer equivalent model to the R-NPs.

The effective RI calculation of all the materials when the R-NPs are fabricated, allows developing a multilayer 1D model equivalent to the 3D photonic structure able to predict the response of R-NPs.

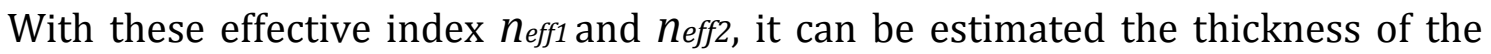
reflectors $(t h)$ to satisfy the lambdaquarter condition for both layers, and the thickness of the cavity.

$$
n_{e f f 1} \cdot t h_{1}=n_{e f f 2} \cdot t h_{2}=\frac{\lambda_{G a p}}{4}
$$


This model based on 1D multilayers was also used for the prediction of the biomolecules attaching the nanoillars surface (figure 39):

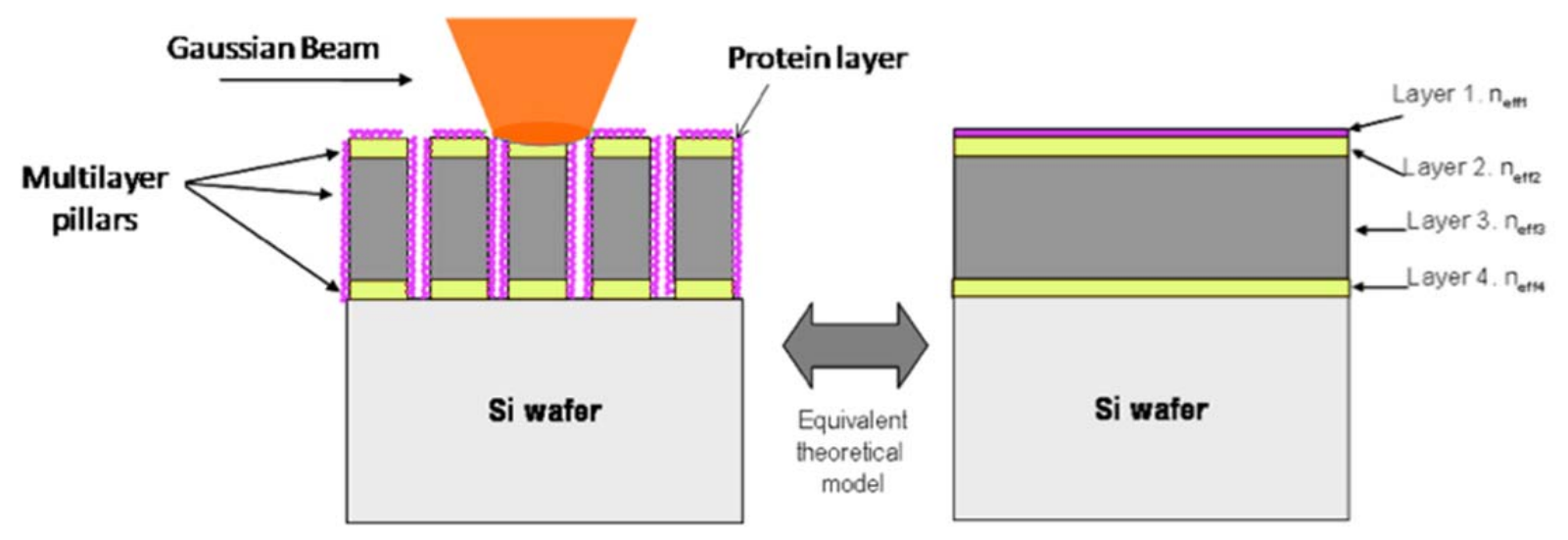

Figure 39: Equivalent model for biolayer estimation over nanopillars. [Casquel, 2012].

\subsubsection{Influence of the Bragg Reflectors number in R-NPs}

The number of Bragg reflector pairs has an important influence on the optical response of the multistack. For example, for a theoretical configuration of $\mathrm{Si}_{3} \mathrm{~N}_{4} / \mathrm{SiO}_{2}$ pairs, with a thickness of $110 \mathrm{~nm}$ (silicon dioxide) and $80 \mathrm{~nm}$ (silicon nitride), there is an optical band gap between 485 and $665 \mathrm{~nm}$, approximately. Considering a central cavity of $210 \mathrm{~nm}$ of silicon dioxide and several numbers of pairs of reflectors, the optical response varies as it is shown in Figure 40. It shows the theoretical optical responses (in this case, optical refection); for different numbers of pair reflectors $(6,10$ and 18 pairs).

The intensity signal is reduced as the number of reflectors is decreased. Besides when looking to figure 40. C, is very striking how the band gap is marked and more defined in simulations for 18 pair of reflectors. This affects directly to the resonant mode sharpness, conditioning the FWHM and thus the sensing quality factor of the structure. 
It is then, demonstrated that it is desirable to have as many reflectors as possible. Unfortunately this is very often limited on one side by the fabrication techniques, being unable to penetrate enough the multistack to generate the nanopillars height estimated. And on the other side it is limited by the physical instabilities appearing in very high aspect ratio (AR) nanopillars. (Big height and small diameter).

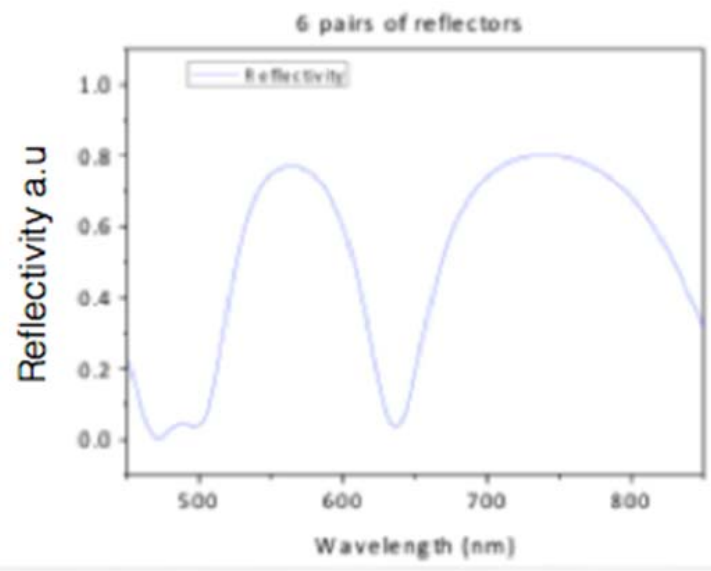

\section{$\mathrm{Si}_{3} \mathrm{~N}_{4} / \mathrm{SiO}_{2} 6$ BR pairs}
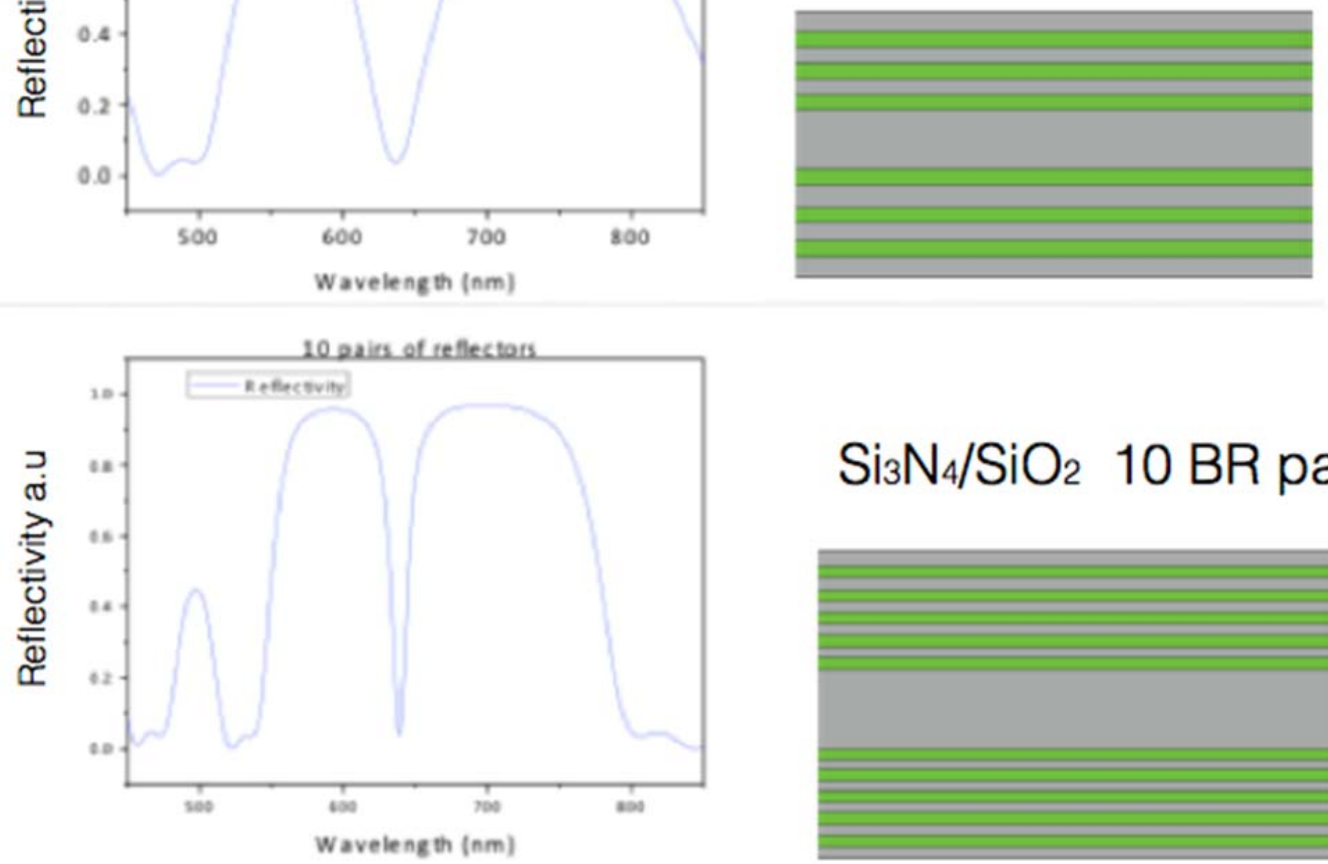

$\mathrm{Si}_{3} \mathrm{~N}_{4} / \mathrm{SiO}_{2} 10$ BR pairs

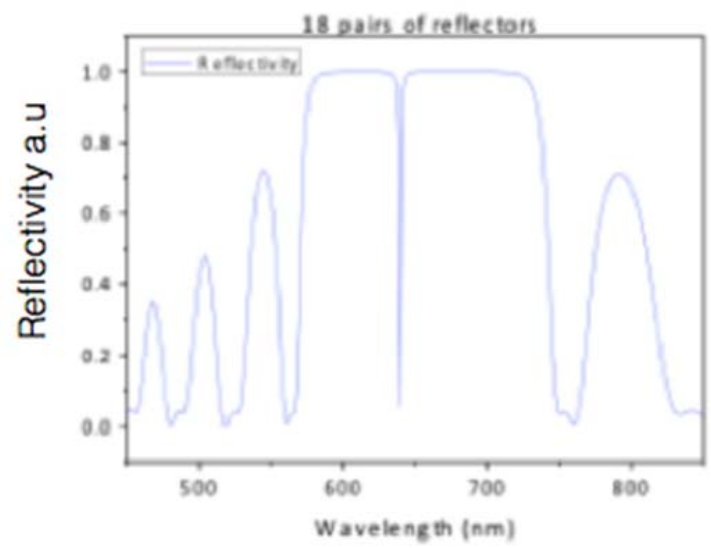

$\mathrm{Si}_{3} \mathrm{~N}_{4} / \mathrm{SiO}_{2} 18$ BR pairs
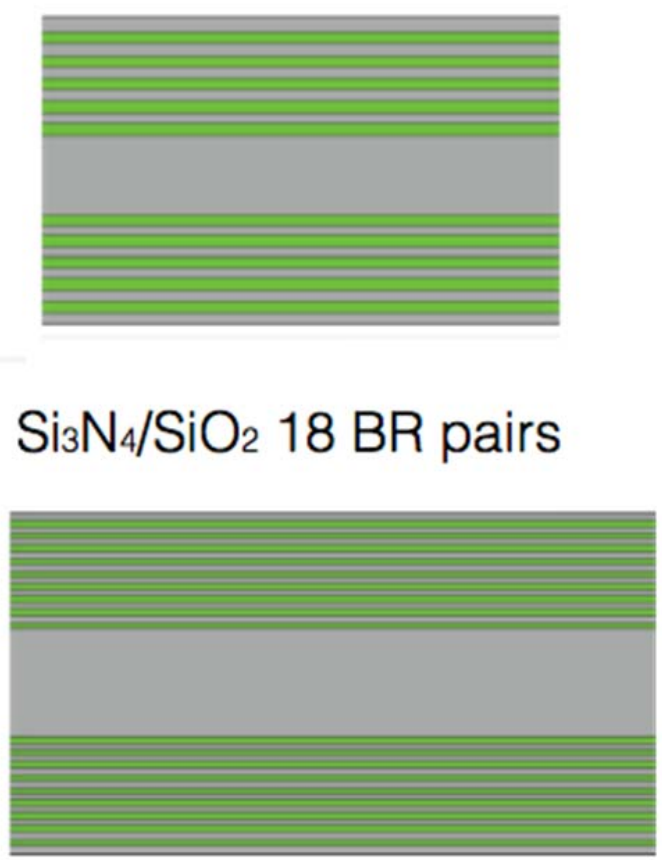

Figure 40: On the left. Optical response of different number of Bragg Reflectors pairs. On the right, drawing of the different Bragg Reflectors pairs. 


\subsection{Summary}

This forth chapter of the work resulted elemental in order to expose the optophysics conditions motivating the use of R-NPs as optical sensors.

It was studied the light confinement in nanopillars based surfaces. Also it was explained the optical properties of the Bragg reflectors and how the theoretical simulations are performed in order to achieve the best optical performance of the R-NPs, taking into account the resources and time available. The influence of the number of reflectors and the lattice parameter was also analyzed.

According to scientific literature nanopillars have been used for biosensing in the past. Nevertheless, the combination of the optical properties conferred by Bragg reflectors materials, and the nanopillars, has been only tested by the GOPB from UPM. 
Chapter 4: Resonant Nanopillars Arrays 


\section{FABRICATION OF R-NPs}

\subsection{General introduction to micro-nano fabrication techniques}

The fabrication of sub-micrometric and nanometric structures such as nanopillars for chemical sensing and biosensing applications have been studied and reported in the literature. [Holgado, 2010], [Kubo, 2011], [Kugel, 2014].

Microsystems technologies (MEMs) is possibly one the breakthrough technologies in the recent years. It has allowed the miniaturization of transducers, such as microsensors and microactuators that can be wholly integrated in single chips. Their resounding success in the microelectronic industry is due to their ability to be mass-produced at a low cost. They have opened the door to a market, until then unexplored, which is expected to grow in investment and research in the coming years.

The nanotechnology goes one step forward, focusing on the manipulation of the materials at an atomic and nanometric level. The production is may not as controlled as MEMs but several advanced techniques (e-beam, LIL, RIE among others) have provided a method of manufacturing with replicated features, among different batches, in mass scale production. These advanced techniques [Chang, 2007], [Liu, 2002], have also enabled the fabrication of structures with different geometries of high aspect ratio (AR) [Trifonov, 2005] in order to enhance the specific sensing surface as well as to take advantage of the optical properties produced by the light confinement in some nanostructures [Chigrin, 2004]. The approaches for the implementation of the nanotechnology go from bottom-up to top-down methodologies which include the material deposition at a nanometric level, as well as surface nano-pattering, through different strategies, and selective etching of the materials. In particular, in the optical biosensors field, these techniques imply the possibility of developing integrated biosensors such as R-NPs 
within a great potential to be used as lab-on-a-chip multiplexed sensors for rapid test analysis.

In order to contextualize the fabrication scheme of RN-Ps, below there are summarized some of the most relevant used fabrication techniques, which are not the focus of this work but worthy to mention in order to provide wider overview of the general fabrication and of the procedures involved in nanotechnology. Even though for a further reading and understanding of the fabrication strategies of nanostructures it can be consulted the thesis work of Dr. Sanza, 2015. [Sanza, 2015]. The described techniques are divided into three different areas: Thin film deposition, lithography and etching.

After this, it is explained the process of R-NPs fabrication for multiplexing biosensing.

\subsubsection{Thin film layer deposition}

For the fabrication of such nano-devices, semi-conductive materials and /or polymeric materials are usually used. They are deposited on the substrates through different strategies known as "thin film layer deposition".

For the polymer resist deposit, the most common strategies are: Spin-coating, spry-coating, meniscus coating. They are briefly described in the figure 41 . [Sanza, 2015].

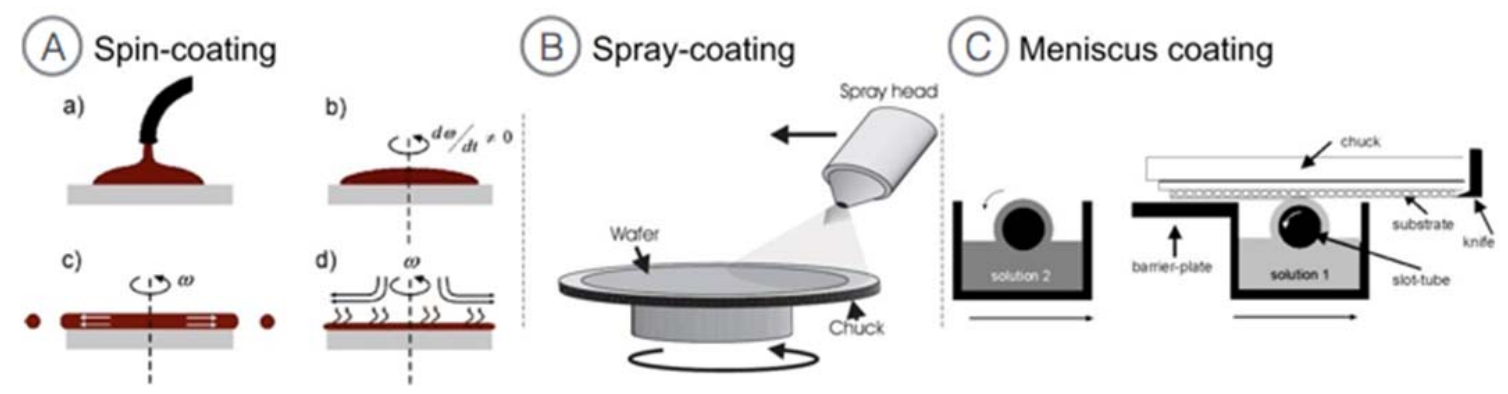

Figure 41: Thin film deposition methodologies. A. Spin coating. B. Spray coating. C. Meniscus coating. [Hellstrom 2007], [Desmaële 2015], [Schmidt, 2000]. 
The Chemical Vapor Deposition CVD is the most popular used technique for wellcontrolled thickness, of semi conductive material deposition. It is a technique based on the exposition of the substrate to two reactive volatile materials that reacts with the surface, growing a thin layer of the produced material over the substrate. (Figure 42). For a nanometric control of the thickness, and sequence of the layers, it has been developed the Atomic Layer Deposition (ALD). [George, 2009], [Leskelä, 2002].

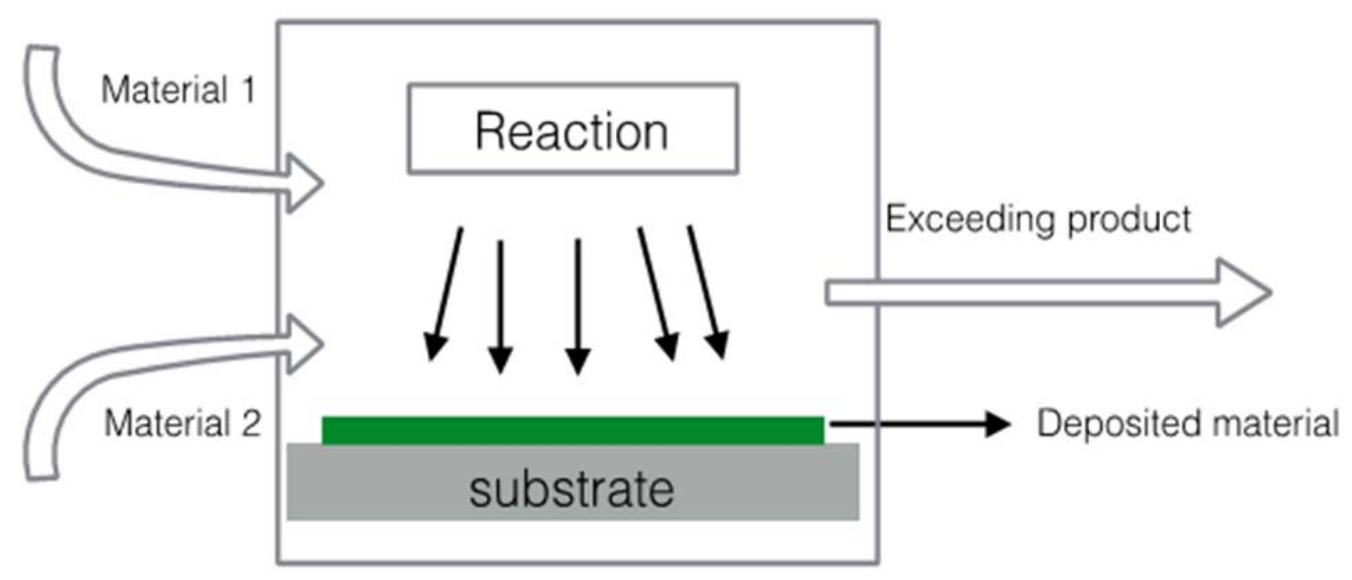

Figure 42: Scheme of the basic functioning of chemical vapor deposition.

For example for the multilayer fabrication of R-NPs it was used CVD technique. $\mathrm{SiO}_{2}$ and $\mathrm{Si}_{3} \mathrm{~N}_{4}$ were deposited sequentially according to the reaction as follows:

$\mathrm{H}_{2} \mathrm{O}+\mathrm{SiCl} 4 \longrightarrow \mathrm{SiO}_{2}+\mathrm{HCl} \quad$ Reaction 1 $3 \mathrm{SiCl}_{2}+4 \mathrm{NH}_{3} \longrightarrow \mathrm{Si}_{3} \mathrm{~N}_{4}+6 \mathrm{HCl}+6 \mathrm{H}_{2} \quad$ Reaction 2

Physical Vacuum Deposition utilizes a vacuum chamber to evaporate and deposit the material on the substrate. The most known and used PVD strategy is probably the sputtering (figure 43). Inside a chamber, energetic particles are pumped into the target material to be deposited. It is energetically charged and the atoms of the material are spattered towards the target substrate, less energetically charged. 


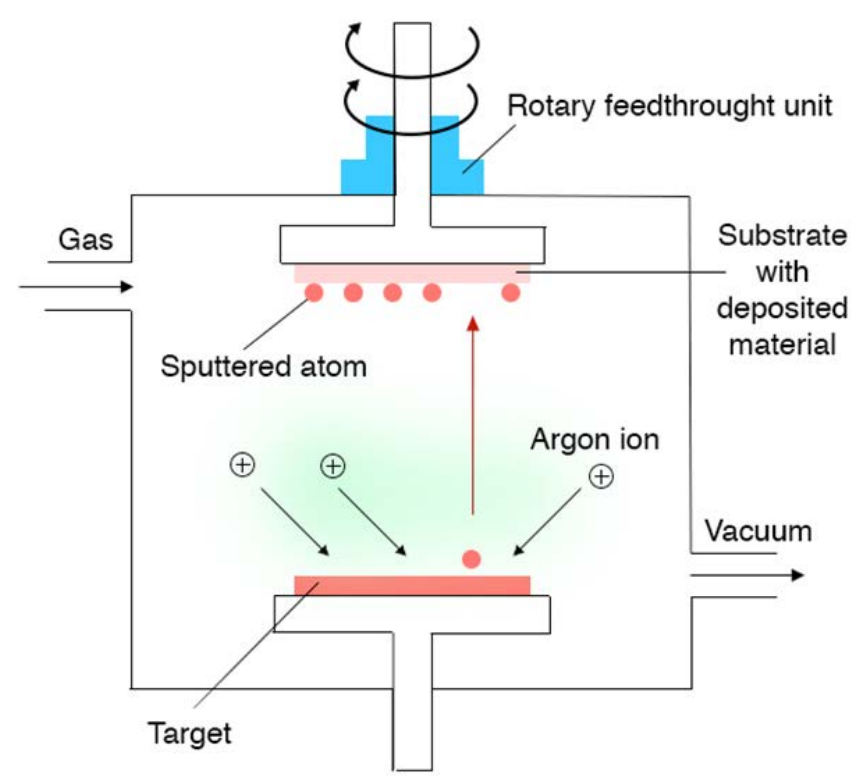

Figure 43: Scheme of sputtering functionement.

\section{1.2 Lithography}

It is a micro-pattering technology widely used in the microelectronic field. It is used to create a specific pattern directly over the surface of the material or over the thin film previously deposited. There exist different ways to perform this:

\subsubsection{Photolithography}

In photolithography it is needed a photo-mask (normally made of polyamide or metal) to define the pattern to be transferred to the surface. Among photolithography techniques, it can be highlighted the contact photolithography, proximity, projection printing system, and immersion photolithography. In the next figure it can be observed the scheme of the four different photolithography techniques mentioned: 


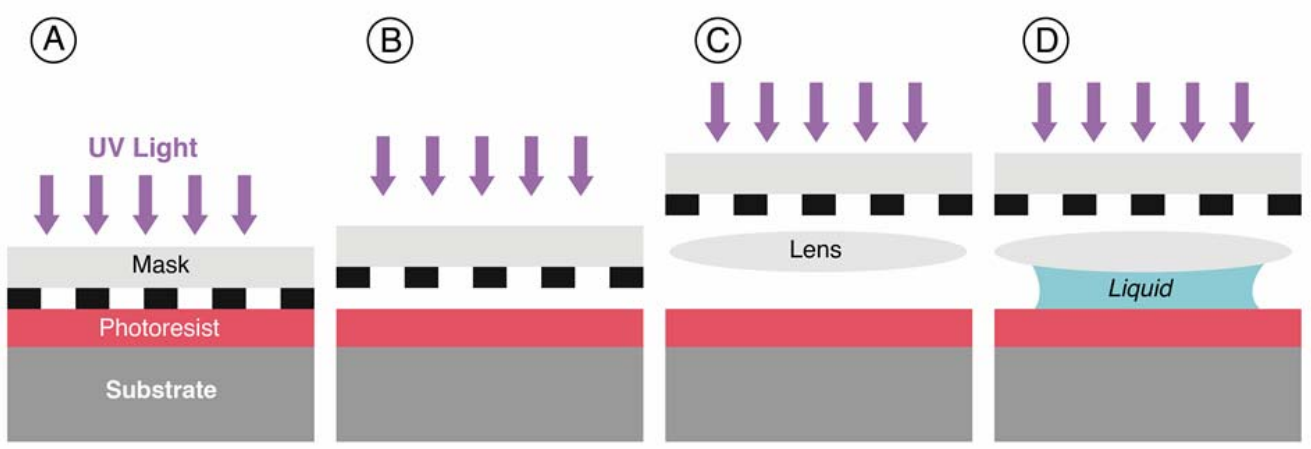

Figure 44: Photolithography methods: A. Contact. B. Proximity. C. Projection. D. Immersion.

Contact lithography has been used by the group of Optics, Photonics and Biophotonics of UPM. [Sanza, 2011].

\subsubsection{Laser lithography}

This technique does not need the use of a photo-mask to draw the pattern, but direct laser writing or laser interference is used to create the pattern.

Some laser techniques used for this purpose are: Laser ablation, selective, twophoton polymerization [Nanoscribe] and interference lithography (LIL). (Figure 45). 

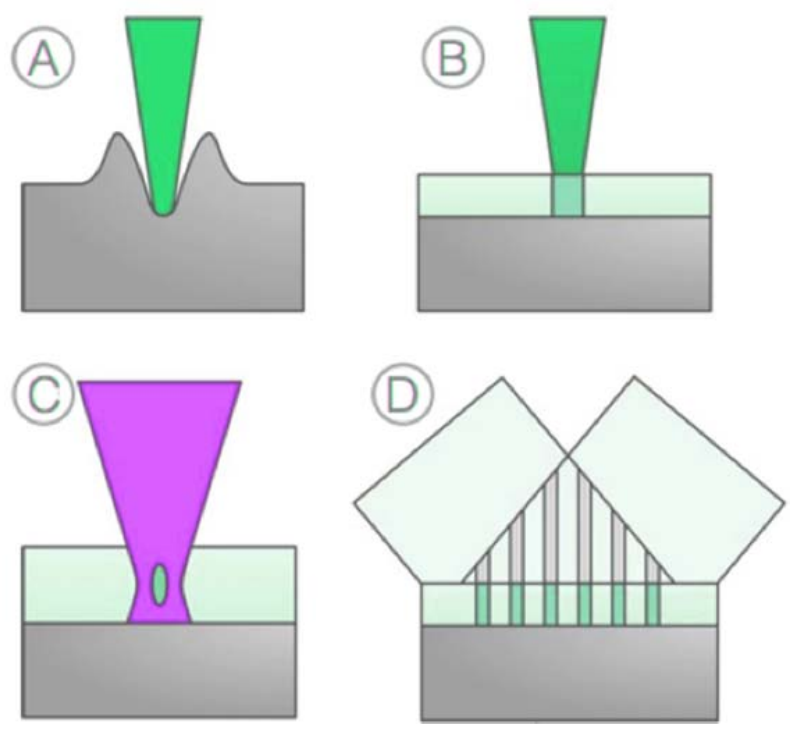

Figure 45: Laser lithography for micro and nano-pattering: A. Laser ablation. B. Selective polymerization. C. Two photon polymerization. D. LIL [Sanza, 2015].

Laser lithography is a very powerful technique able to act over biomaterials for surface biofunctionalization, or for self-cleaning material purposes. [Yu, 2005],[Berendsen, 2009].

R-NPs pattern was fabricated by means of laser interference lithography (LIL). In this technique two lasers with the exact same wavelength generate an interference, which creates a pattering over the surface. Lattice parameter or separation of structures $(\Lambda)$ is defined by equation 18 where $\lambda$ is the laser wavelength, $n$ is the refractive index of the material and $\alpha$ is the angle of incidence of the laser beam. [Sanza, 2015] [Päivänranta, 2011] [Lasagni, 2010].

$$
\Lambda=\frac{\lambda}{2 n \sin (\alpha)}
$$

This allows an accurate definition of the geometric structures to be developed over the surface. The pattern is drawn commonly on a specific multistack of 
photoresists, which needs to be engraved into the final substrate. This transfer of pattering is commonly accomplished by wet or dry etching. It is a low cost technique that can be used in in big areas with a homogeneous pattern.

\subsubsection{E-beam}

Electron-beam (e-beam) is another mask-less technology used for direct writing. It is based on a focused electron beam, with a high current density incising an electron sensitive resist. [Pease, 2006]. PMMA is one of the most popular resist used for e-beam lithography showing good resolutions results. [Elsner, 2001]. Also SU-8 photoresist has been used for this technique under the purpose of biosensing applications [López-Romero, 2010]. Although better resolution results are obtained with e-beam than in photolithography. The electron scattering in e-beam, limits the maximum resolution achievable with this technique. It is essential to remark that this technique is also often used for layer deposition, in fact this e-beam evaporator is involved in the R-NPs fabrication, for the SiO2 interlayer deposition, as it will be explained later in this chapter.

\subsubsection{Mechanical pattering}

By fabricating large molds to work at a wafer scale, this technique is presented as very interesting option to be applied at industrial level. Also this is a low cost technique because avoids several steps implied in photo and laser lithography since it is not needed the use of chemical solvent or resist developers.

Nanoimprinting lithography (NIL) use molds or stamps fabricated with the pattern desired and transferred into a soft material. For this reason the materials are firstly heated in order to reach every pattern cranny. [Chou, 1995]. The main drawback of this technique is the need of use of a stamp, which in combination with the material to engrave limits the pattering resolution. 


\subsubsection{Etching}

The final goal of the fabrication processes is to obtain a surface defined with the desired layout or structures over the chosen material. After the lithography pattering methods, the motives have to be engraved in the surface by removing solid material on the surface. This can be accomplished by wet of dry etching.

- Wet etching

In wet etching the substrates are immersed into a chemical solution attacking selectively or not, (depending on the chemicals of the solution and the materials on the substrate) certain materials on the substrate. This can be performed in mass production to produce several batches at once and it is not needed a sophisticated equipment to do it.

Isotropic etching attacks the material in all directions because the molecules of the etching solution move in every direction. The solutions are mixed in different concentrations according to the desired etching rate, some of the are: $\mathrm{HF}$ for $\mathrm{SiO}_{2}$, $\mathrm{H}_{3} \mathrm{PO}_{4}$ for $\mathrm{Si}_{3} \mathrm{~N}_{4}, \mathrm{HNO}_{3}$ for aluminum, $\mathrm{NH}_{4} \mathrm{OH}$ for titanium, etc. There is a great amount of references in the scientific literatures regarding this widely used technique. It is commonly used to remove entire layers from a substrate.

Anisotropic etching attacks the material in one single direction, defining specific structures. There are chemical solutions that attack different materials at a different rate, providing the capacity of performing an anisotropic wet etching. Nevertheless for anisotropic etching it is more effective to perform a dry etching.

- Dry etching

The substrate is exposed to the bombardment of ions from different materials. It is less selective than wet etching but is more commonly used for anisotropic etching were the motives need to be accurately engraved. The disadvantage of this technique is that it is more expensive and needs specific equipment's with a 
vacuum chamber to be accomplished, nevertheless the operating of these equipment's are easy and fast to learn and don't need specialist to handle them.

Plasma etching and Reactive ion etching or RIE are the most well known techniques of dry etching. RIE uses a mix of gases that are pumped into the vacuum chamber and due to the difference of potential between ions and the surface of the substrate, it is created an electric field through which ions precipitate into surface removing the etching sensing area.

For the R-NPs fabrication RIE was involved after LIL lithography, and after that, a chromium layer deposition was performed.

The combination of all the techniques aforementioned have led to the development of nanostructures and nano-circuits that have permitted the process of miniaturization so pursued in the technological industry.

\subsection{Fabrication process of R-NPs}

The optical response of the R-NPs mainly depends on the geometrical parameters of the nanopillars and the optical properties of the materials used and the distribution of materials (film stack). [Cornago, 2016]. As stated in the former chapter the main advantage of the use of R-NPs as label-free biosensors is due to the formation of a well-defined resonant mode when they are optically interrogated, which is sensitive to changes in the RI of the media. It is essential that the fabrication does not only imply the development of the R-NPs but also their distribution in independent cells or arrays, thus guaranting the multiplexing properties of this type of nanosensors.

First of all it was designed a multistack of the 10 pairs of $\mathrm{SiO}_{2} / \mathrm{Si}_{3} \mathrm{~N}_{4}$ Bragg reflectors and a $\mathrm{SiO}_{2}$ central cavity. The thickness of each layer of $\mathrm{SiO}_{2}$ was $110 \mathrm{~nm}$, and the thickness of each layer of $\mathrm{Si}_{3} \mathrm{~N}_{4}$ was $80 \mathrm{~nm}$. The thickness of the cavity of $\mathrm{SiO}_{2}$ was $210 \mathrm{~nm}$. Therefore total coating was $2110 \mathrm{~nm}$. This multilayer stack was prepared by CVD in Fraunhofer Research Organization over a quartz substrate of 
$500 \mu \mathrm{m}$ thick. (Figure $46 \mathrm{~A}$ and B). Once the multilayers were prepared, squared wafers of $86 \mathrm{~mm}^{2}$ of area with the multilayers deposited (figure 46.A) were shipped to the GOPB from UPM for the chip dicing according to the different requirements. Wafers were cut in three different sizes:

$-14 \times 26 \mathrm{~mm}$

$-14 \times 20 m m$

$-42 \times 26 m m$

There are several tools to do the dicing of the substrates. At the beginning wafer were diced by thermally induced stress in a ML-100 workstation with an excimer laser $\mathrm{KrF}$ at $248 \mathrm{~nm}$ (Figure 46.C) in the "Centro Laser" from UPM. Even though this cutting technique was not appropriate for quartz substrates because it resulted too time consuming for Project requirements. For that reason it was decided to use a different method for the wafers dicing.
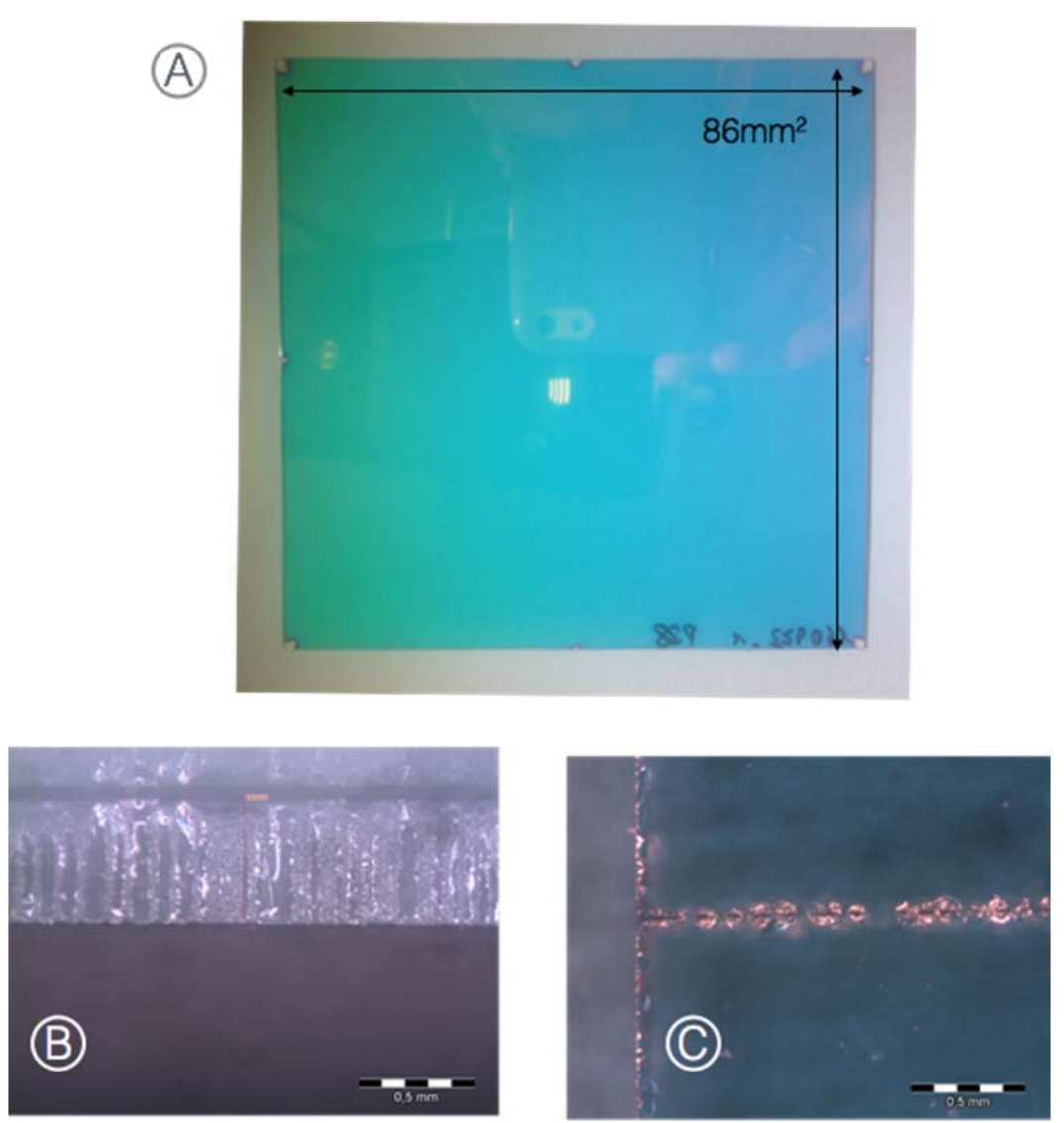

Figure 46: A. Squared wafer with the multilayers deposited. B. Cross view of the wafer. C. Wafers cut under excimer laser. 
Thus, wafers were easily cut within a diamond saw (Figure 46.B) in The "Instituto de Sistemas Optoelectrónicos y Microtecnología" (ISOM). In figure 47 there are shown different dicing techniques.
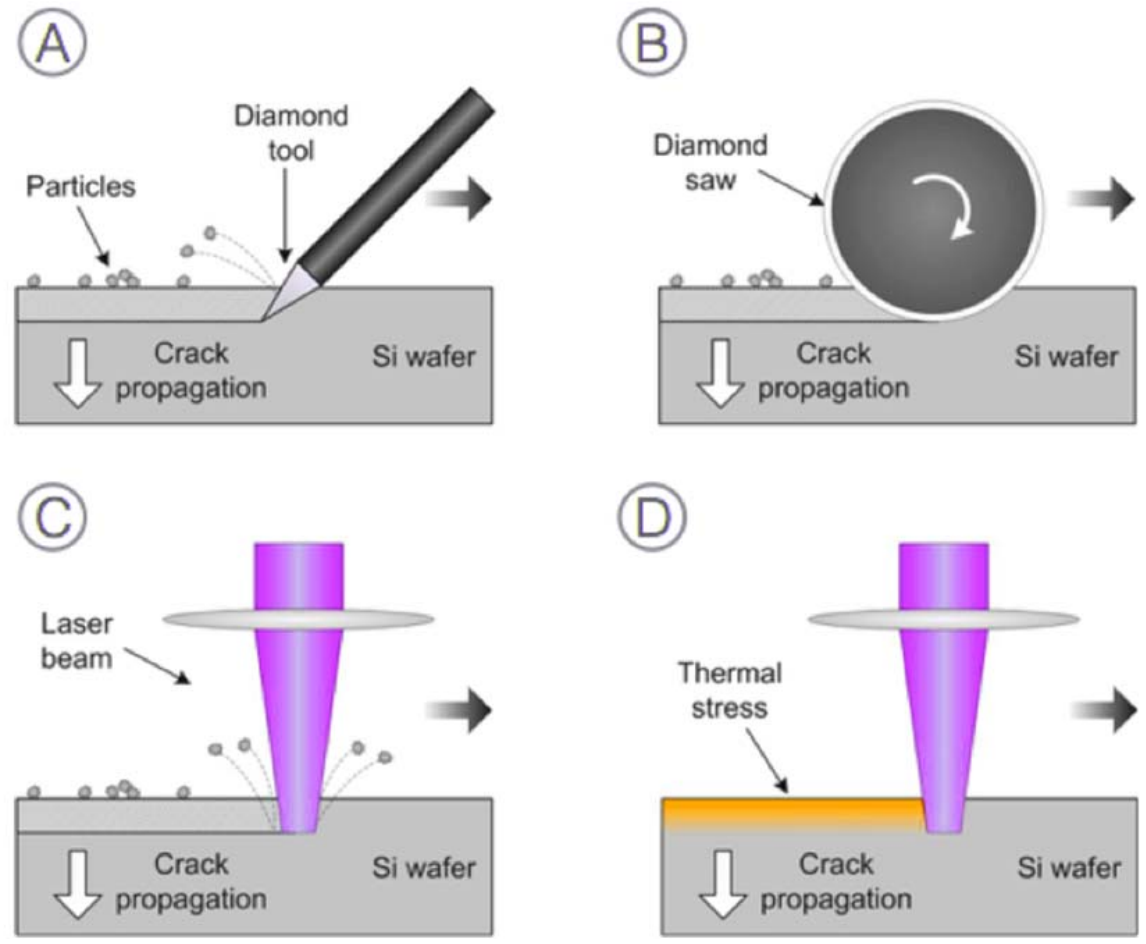

Figure 47: Dicing techniques: A. Diamond pencil. B. Diamond saw. C. Laser beam. D. Thermal stress. [Sanza, 2015].

After dicing the chips, they were sent to Cemitec for the fabrication of the R-NPs with multiplexing capabilities. Different diameters and lattice parameters were fabricated in order to know which was the ideal specific conformation of R-NPs, to increase sensitivity according to the Enviguards Project requirements.

The first step of the fabrication of the R-NPs (Figure 48) over the mulistack was the preparation of an adequate stack to create a pattern with a Lloyd's mirror LIL setup with a He-Cd laser (325 nm). For this purpose there were deposited an antireflective coating (ARC, XHRiC-16 from Brewer Science), and a negative photoresist layer (PRL, NR7 from Futurrex) by using a spin coater (WS-650S6NPP, Laurell). $\mathrm{A} \mathrm{SiO}_{2}$ interlayer between ARC and PRL was necessary for a vertical pattern transference and also because it provides a suitable profile for 
lift-off (additive method to selectively define the geometry of the materials). (Figure 48. B) $\mathrm{SiO}_{2}$ interlayer was deposited by e-beam evaporator (Classic 5000, Pfeiffer). [Savas, 1996].

Then a pattern of nanometric holes in a quadratic lattice was created over PRL by exposing samples twice in a Lloyd's mirror set-up. (Figure 48. C). By adjusting the exposure dose of the LIL there were obtained holes diameters between 150 and $250 \mathrm{~nm}$. [Smith, 2001]. The pattern was then transferred from the PRL to the $\mathrm{SiO}_{2}$ interlayer and from the $\mathrm{SiO}_{2}$ to the ARC by RIE (Plasmalab 80, Oxford Instruments) using fluorine gases like $\mathrm{CHF}_{3}$ (fluoroform), $\mathrm{CF}_{4}$ (tetrafluoromethane) and $\mathrm{SF}_{6}$ (sulfur hexafluoride), and oxygen respectively. (48. D) [Cornago, 2016]. A Cr layer of $150 \mathrm{~nm}$ was deposited by e-beam over the patterned surface and immersed in a solution called SC1 of RCA for lift-off process. In this step it was included the polyimide mask for the arrays distribution of the R-NPs (Figure 51. A). [Kern, 1990]. This process leads to a pattern of Cr pillars (Figure 48. E), which are used as a hard mask for the anisotropic RIE of the nanopillars. Nanopillars then resulted with a Cr layer on their surface, but it was removed with a commercial specific etching process. (Figure 48. F). 


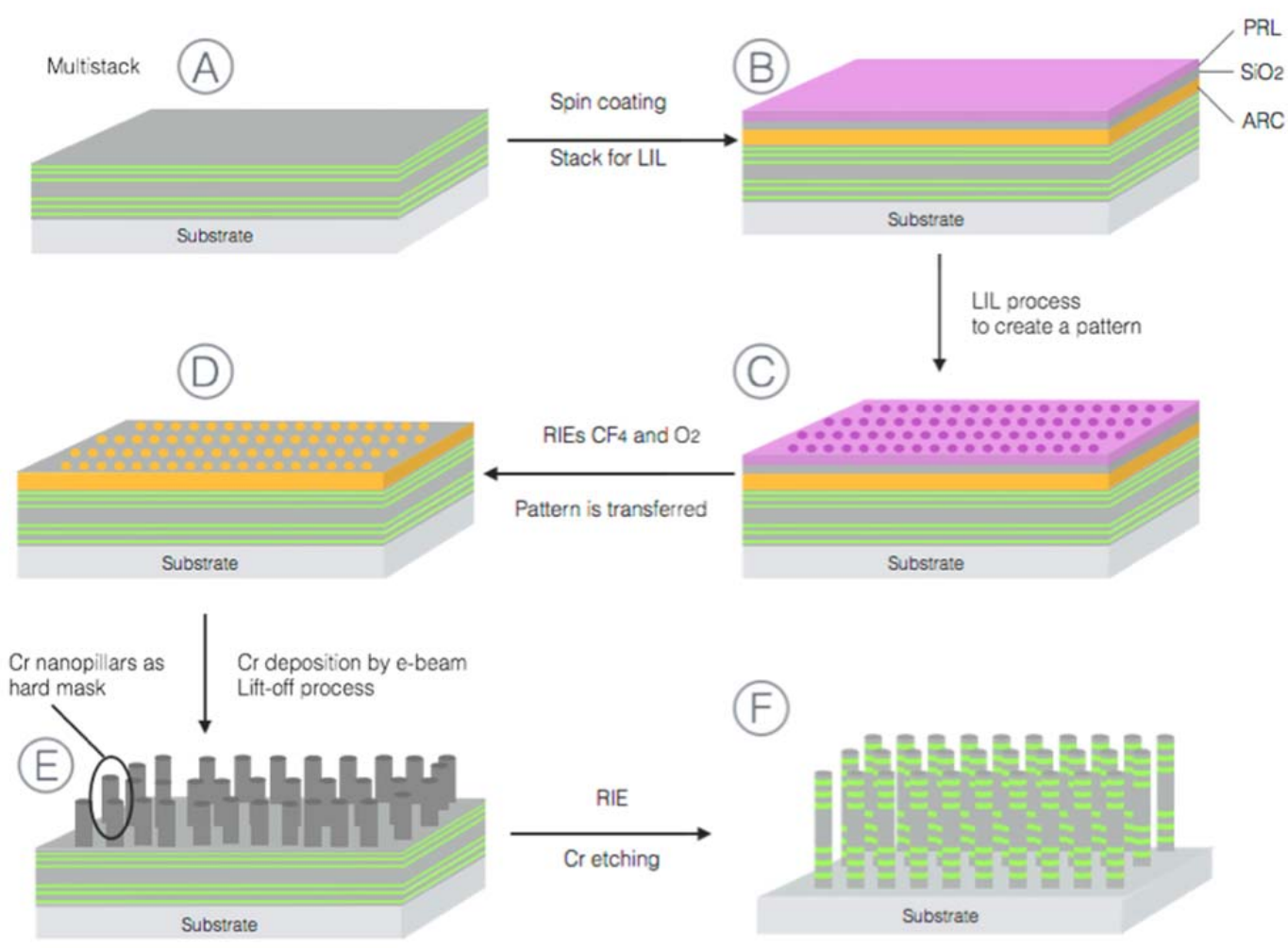

Figure 48: Fabrication steps of the R-NPs. A. Multistack. B. Stack deposition for LIL. C. Pattern after LIL. D. Transferred pattern with RIE. E. Cr hard mask for RIE. F. R-NPs.

The figure 49 shows a very detailed SEM picture of one of the samples of R-NPs of $400 \mathrm{~nm}$ of lattice parameter. The $\mathrm{Cr}$ etching did not fully remove the $\mathrm{Cr}$ layer. That is the reason why the R-NPs present an oval and darker area on the top of the pillar. The maximum height is determined by the multilayer thickness, and it oscillates between 2026 and $2031 \mathrm{~nm}$ from one pillar to another. This variation depends mainly on the RIE homogeneity process along the chip surface. Unlike thickness, diameter is a parameter that fluctuates, not only between the nanopillars but also along the same pillar. As observed in SEM photo R-NPs have a baseball bat shape in which diameter varies from $162.4 \mathrm{~nm}$ in the middle height, and $231.5 \mathrm{~nm}$ on the top area. 


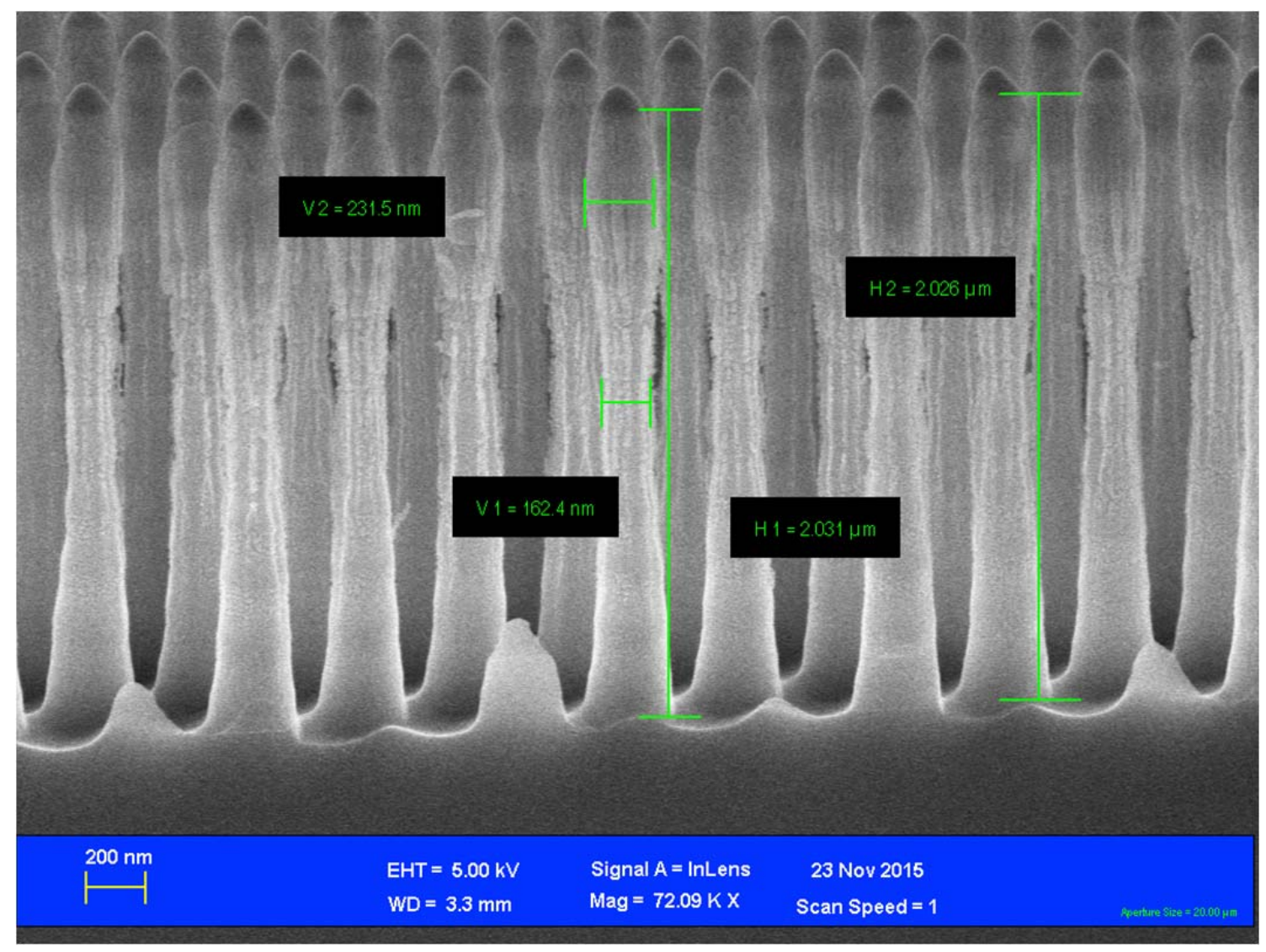

Figure 49: SEM photo of R-NPs with $400 \mathrm{~nm}$ of lattice parameter.

In figure 50 it is represented a scheme of the vertical composition of R-NPs: 10 pairs of Bragg reflectors and a central cavity. In the SEM picture, if looking carefully, it can be identified the different resonant layers. 


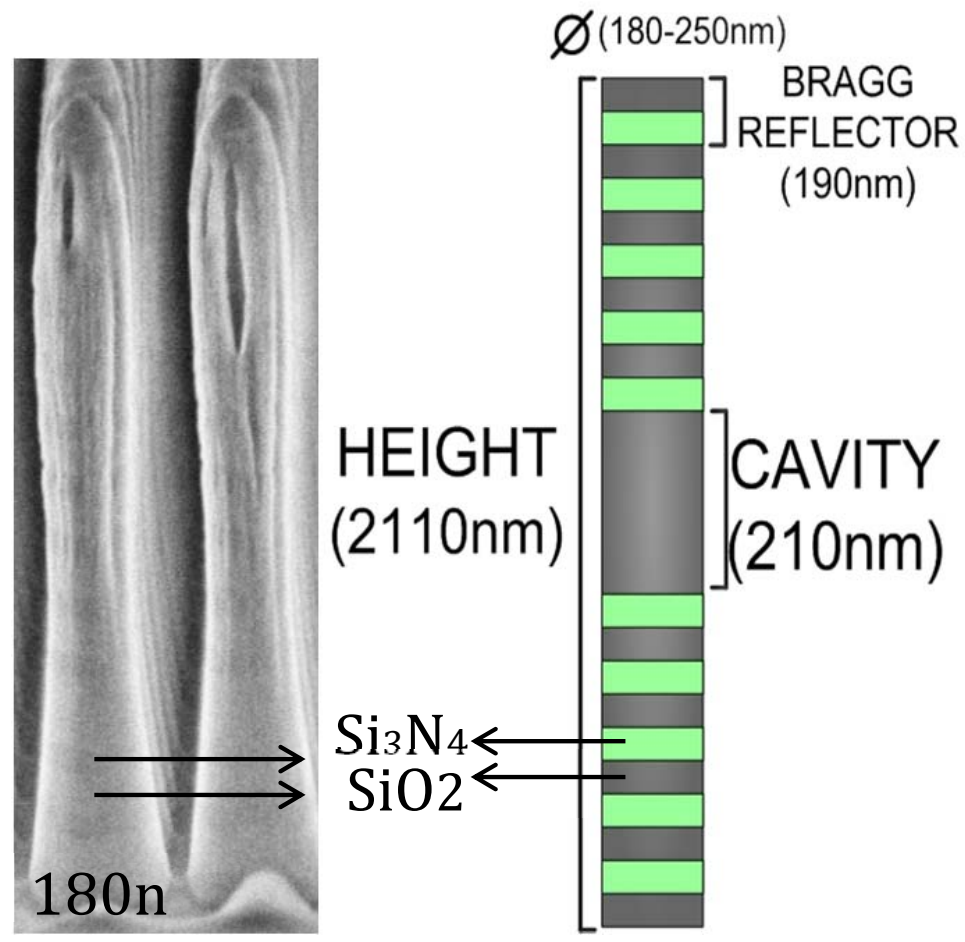

Figure 50: On the left. SEM image of R-NPs.On the right. Schematic draw of a R-NP composition.

For the multiplexing characteristics of the chip sensor, during the fabrication process it was used a polyimide mask containing eight empty squares which defined the R-NPs arrays distribution over the chip (Figure 51. A). This means that R-NPs were not fabricated over the whole surface of substrate, but only on the multiplexing designed areas. (Figure 51). Thus each of the arrays could be independently interrogated and used as a single sensor. The size of the arrays was $1 \mathrm{~mm}^{2}$. For the naming of the different arrays in this work it was used, a coordinate system traditionally used in the Group of Optics Photonics and Biophotonics. Since the chip consist on two rows and four columns, to refer each cell there were used two digits. First referring to the row and the second appealing to the column. Taking as a reference the lower left array (figure 51. B). 

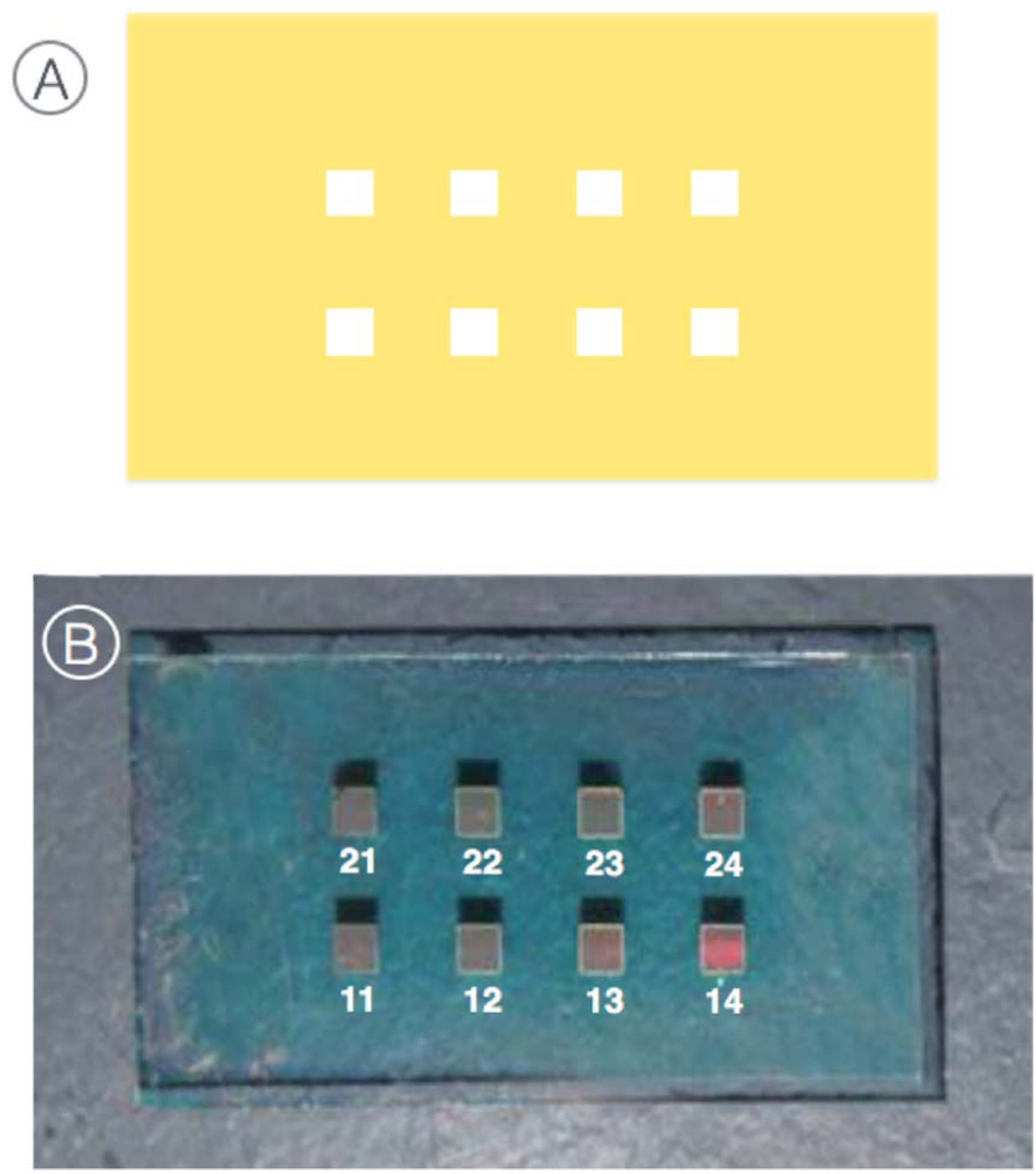

Figure 51: A. Polyimide mask containing eight different cells. B. Photo of a chip with eight BICELLS.

After the fabrication of the R-NPs samples in Cenitec, they were sent to the Group of Optics, Photonics and Biophotonics from UPM for their characterization and experimental analysis.

The fabrication process was reported in the scientific literature. [Cornago, 2016].

\subsection{Summary}

The purpose of this section was to explain the fabrication of the R-NPs and to expose that despite its apparent complexity, it is a reproducible process, which is able to provide multiplexing properties, enabling R-NPs to be used as a label free optical biosensor for mutilanalyte detection at the same time. 


\section{OPTICAL INTERROGATION OF R-NPS}

\subsection{Optical interrogation (holder ad-hoc)}

The advantages of R-NPs as high sensitivity label-free optical biosensors do not only rely on the specific characteristics of their materials and composition; but the characterization procedure is a key factor of their optical performance. In order to take advantage of their optical properties they must be optically, vertically interrogated, so that an evanescence field is created among the pillars, as seen in chapter 4.

The R-NPs samples studied in this work were interrogated from the backside substrate with the R-NPs facing down, so they could be in contact with the surrounding media (Figure 52.B). For this purpose Biod kindly used a 3D printer to fabricate the R-NPs chip holder ad-hoc. (Figure 52.A) This ensures that each array could be properly infiltrated with the liquid introduced into the holder. The setup guaranties the multiplexing properties of the sample chip, because it allows obtaining a separate spectral response for each array by collecting the reflection from the backside of the substrate.
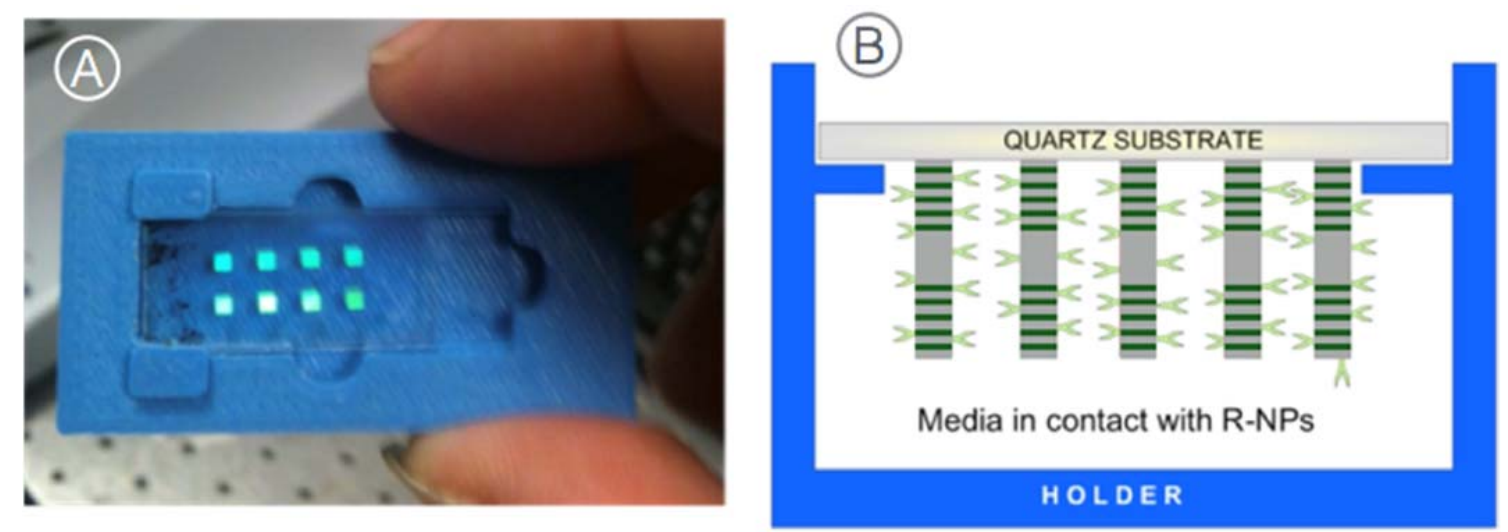

Figure 52: A. Photo of a chip with R-NPs in the holder. B. Schematic representation of the disposition of R-NPs in the holder for the optical interrogation. 


\subsection{Optical interrogation with FT-IR}

At the first stage of the work, in order to characterize the optical response of each array of RNPs independently, it was used a high-resolution FT-VIS-NIR Spectrometer (Bruker VERTEX 70) [Sanza, 2011], [Laguna, 2014a]. A photo of this optical integration setup can be observed in figure 53.A. It was employed an optical objective with a magnification of $4 \times$ at normal incidence, and the spectra were carried out with a resolution of $1 \mathrm{~cm}^{-1}$ and 200 scans, as previously reported in the literature [Holagado, 2010], [Laguna, 2014b] (Figure 53.C). Since the dimensions of the arrays are $1 \mathrm{~mm} \times 1 \mathrm{~mm}$, it was adjusted the beam spot size to fit this dimension by the Hyperion microscope edge apertures attached to the spectrometer. (Figure 53).
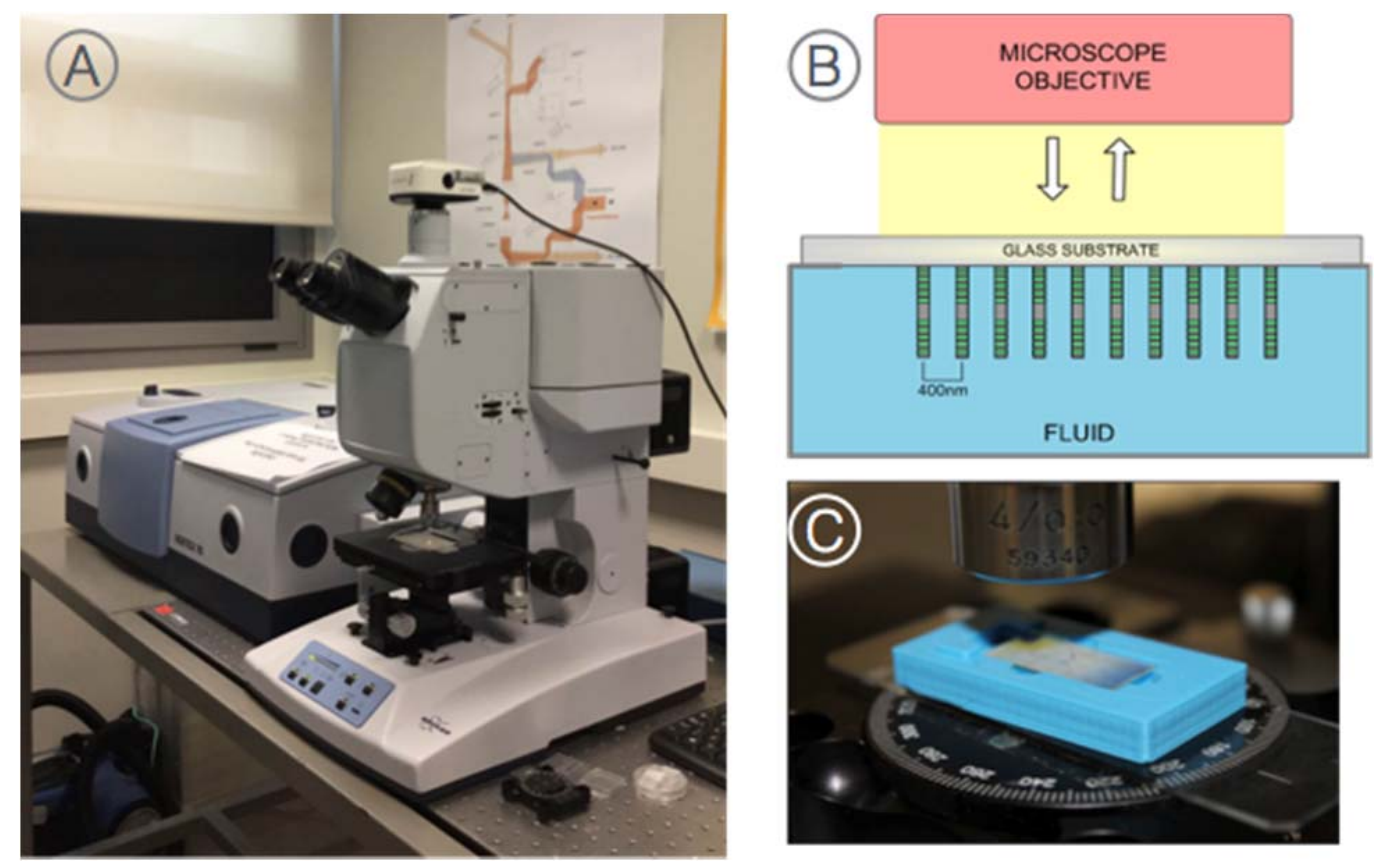

Figure 53: A. FT-IR equipment. B. Schematic representation of the optical interrogation of the R-NPs with the FT-IR. C. Photo of the R-NPs being interrogated with the FT-IR.

The chip was placed on the holder (figure 53.B) with the arrays of R-NPs facing down in order to be in contact with the liquid. 
FT-IR has an optical signal management program called OPUS by default. But for the data processing, it was used a unified system, applied in the GOPB, designed with programs and functions of Matlab, developed by doctor Lavin. [Lavin, 2016]

\subsection{Optical set-up read-out system}

In order to facilitate the interrogation of the R-NPs, it was integrated a specific setup for the optical interrogation of R-NPs at the GOPB facilities.

This optical set up was handier and allowed to change the interrogated BICELLs, and focus them, in a very easy and fast mode. Since its incorporation to the laboratory facilities, all the experiments were accomplished with this optical setup system. It was coupled either to the holder fabricated ad-hoc for static experiments or to the flow cell from fluidic system for continuous flow experiments.

For that reason there were purchased several optical devices and tools:

-Aluminum Optical Breadboard, 300 mm x 600 mm x 12.7 mm, M6 Taps, -XYZ Translation Stage with Standard Micrometers, for the positioning of the BICELLS,

-An optical fiber holder from Filmetrics

-Migtex spectrometer HRS-VIS-005 (covering from 390 to 870 wavelenght of the electromagnetic spectral range).

-Fiber bundle 

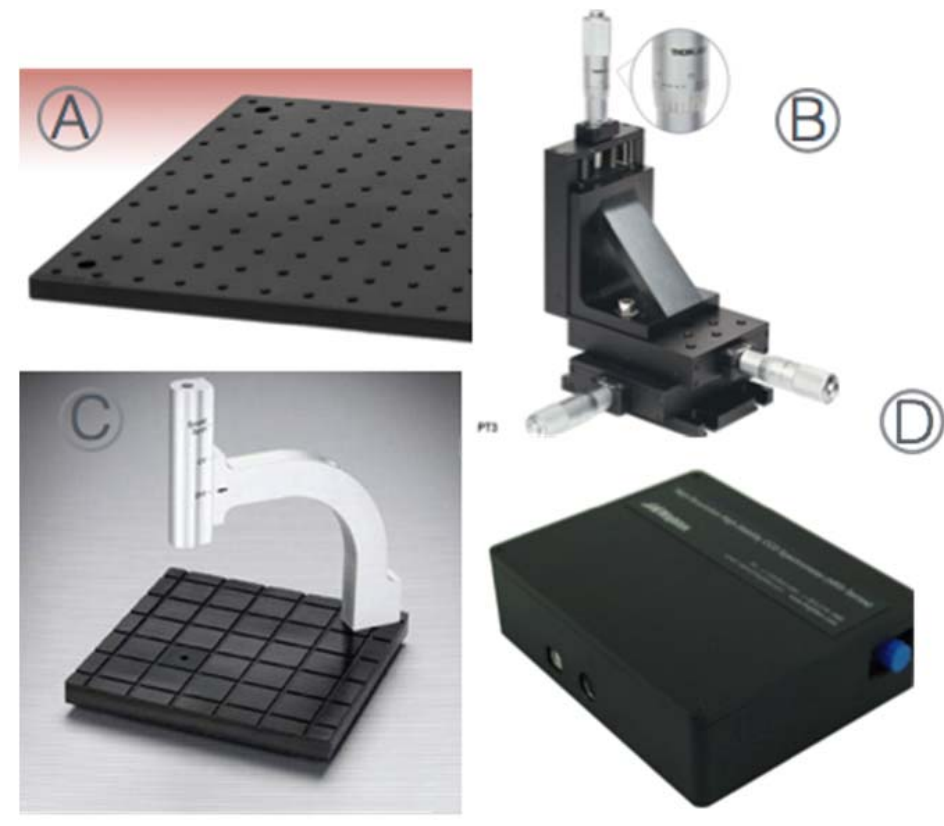

Figure 54: Element of the optical set-up system. A. Optical board with holes. B. Micropositioner $X, Y, Z$. C. Fiber holder with a lent for small spot focusing. D. Espectrometer.

Then the different components were integrated in the laboratory of the GOPB. (Figure 55 and 56). This set-up allows each BICELL to be optically interrogated through a bundle of fiber coupled to and fiber holder, located in a $\mathrm{X}, \mathrm{Y}$ and $\mathrm{Z}$ micropositioner, attached to a track or guide which is on the top of the optical board. (Figure 56). This enables the positioning and focusing of single BICELLs, for multiplexing measurements. Therefore, each of the BICELLs can be vertically interrogated from the backside of the substrate (with the pillars facing down) with the bundle of fibers, which integrates the optical illumination on the outer side (with a diameter of $500 \mu \mathrm{m}$ ), coming from the light source, and the response acquisition in the central area of the bundle. Collected data are addressed to the spectrometer HRS-VIS-005 from Mightex and processed by the program developed by doctor Lavin. [Hernandez, 2015]. The next figure represents a graphic scheme of the layout optical interrogation described. 


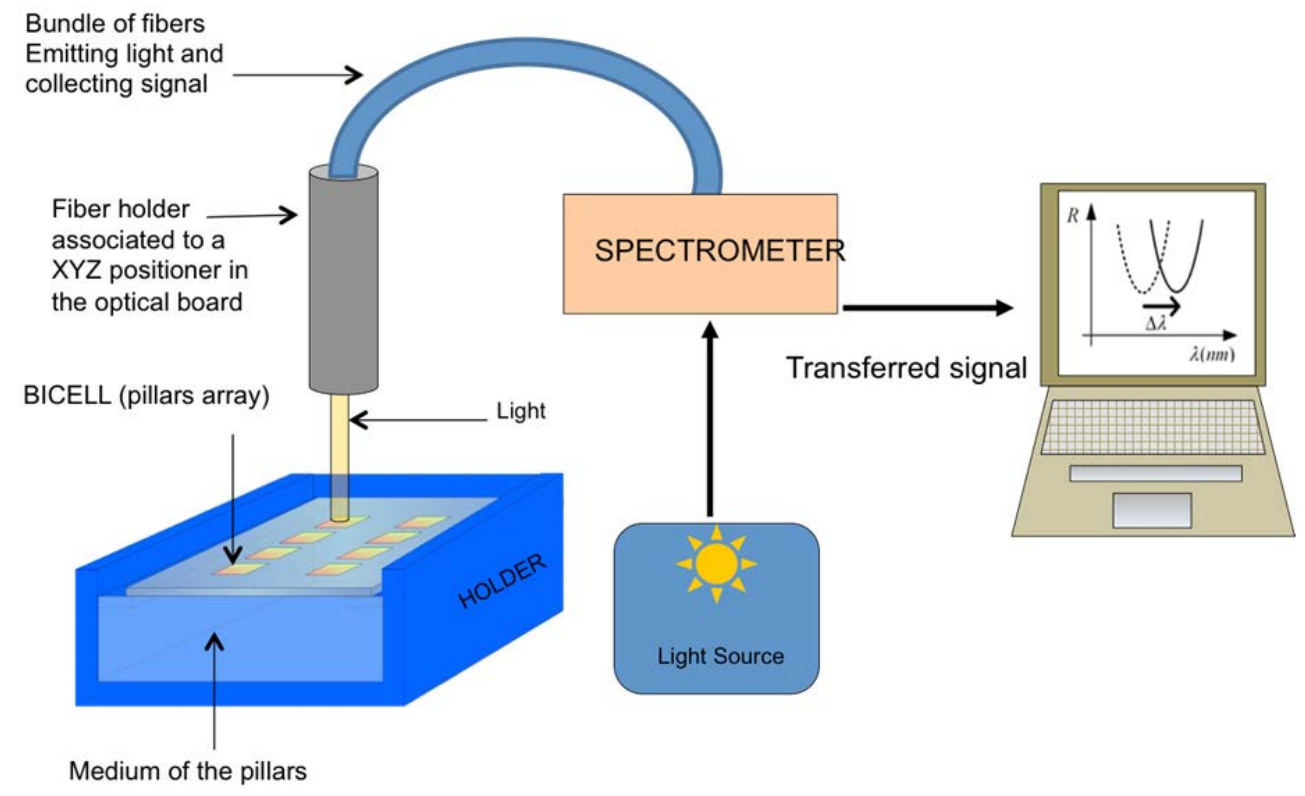

Figure 55: Scheme of the optical set-up operationally.

Next figure is a real photograph of the optical system mounted in the laboratory:

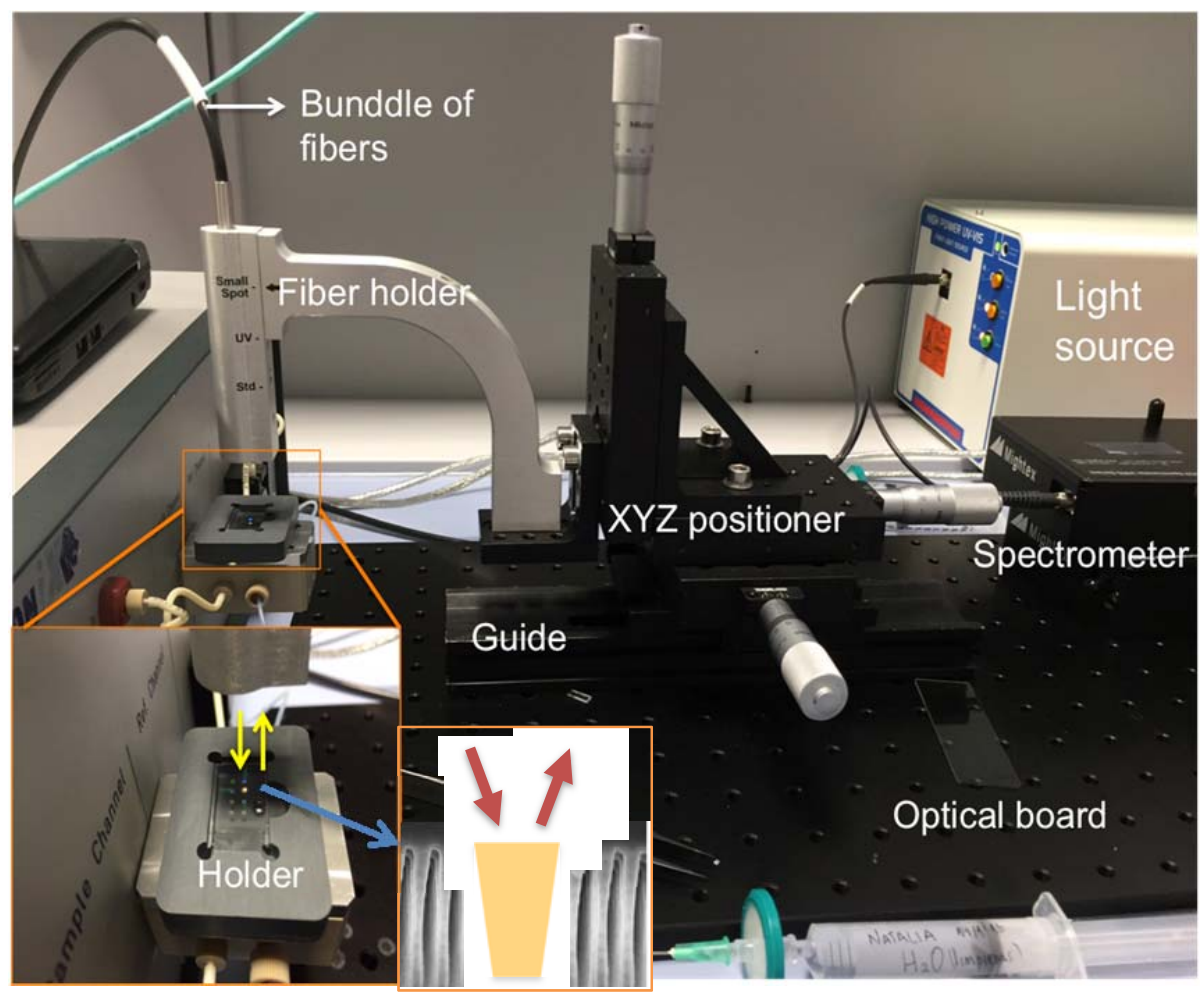

Figure 56: Photo of the optical set-up system mounted in the laboratory of the GOPB. 


\subsection{Fluidic system coupled to the optical set-up read-out system}

For the multianalyte, and real-time measurements, a multiplexed optical transducer with eight BICELLs was integrated in a sealed flow cell with two microfluidic channels so BICELLs could be simultaneously interrogated for multianalyte detection. Lionix developed the fluidic chip holder or flow cell, as well as the fluidic system. [LioniX].

The microfluidic chip was developed for holding chips with eight BICELLs distributed in two channels. It consisted of a chip holder body and a lid, between which the sensing chip, the viton and the fluidic chip were all clamped together. $O$-rings were underneath this package to provide seals towards the chip holderbody. In the next figure (figure 57) there are shown different views of the chip holder and detailed components are presented.
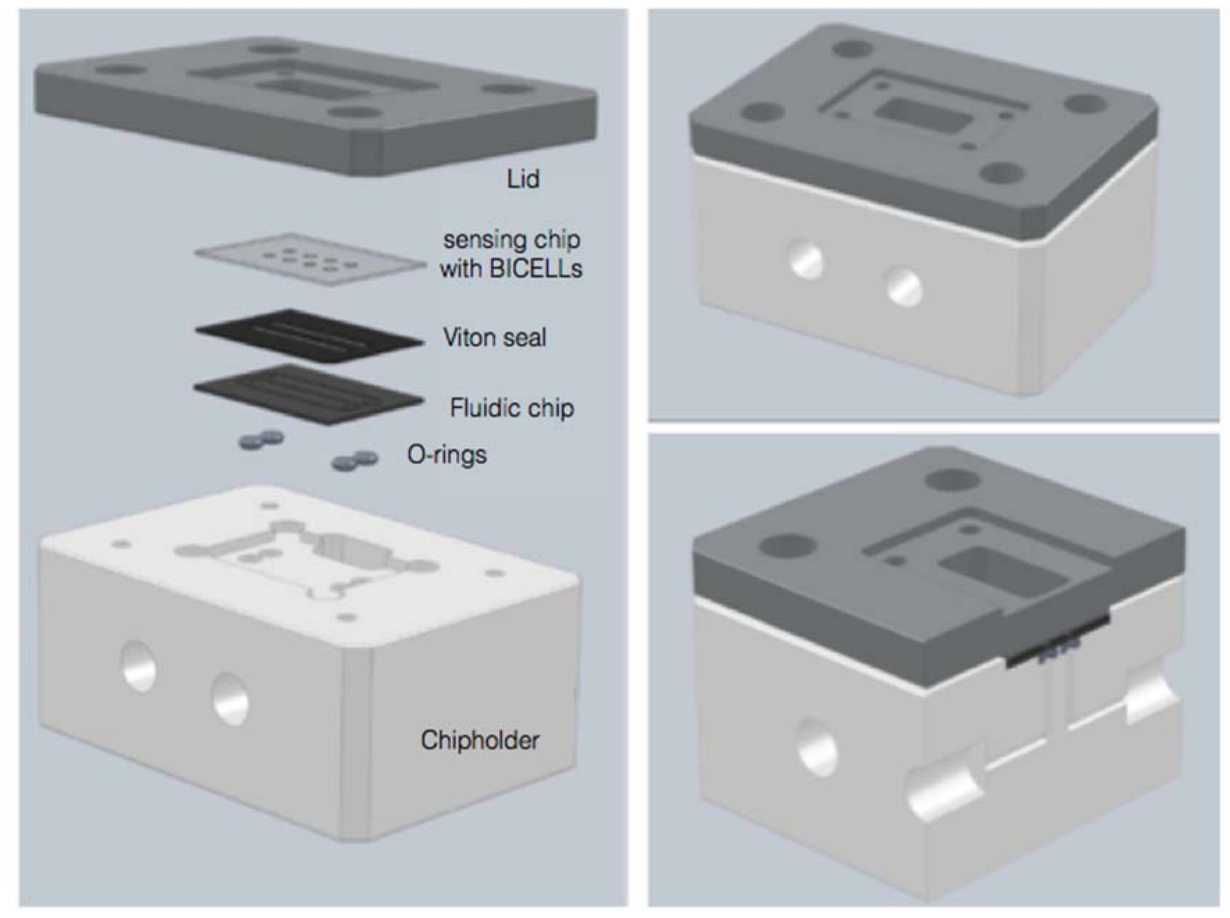

Figure 57: Fluidic holder with all the elements included.

The two different fluidic channels were conceived to be used independently. One for the sample test, and the other, for the reference test, in order to make sure the adequate functioning of the whole system. (Figure 58.B). Each channel was associated to one peristaltic pump (Figure 58.A-2 and 59.A-2), which flows fluids 
from reservoirs (figure 58.A-4 and 59.A-4), selected through two different rotatory multivalves (figure 58.A-3 and 59.A-3). Both channels fluid ends up in a waste reservoir.
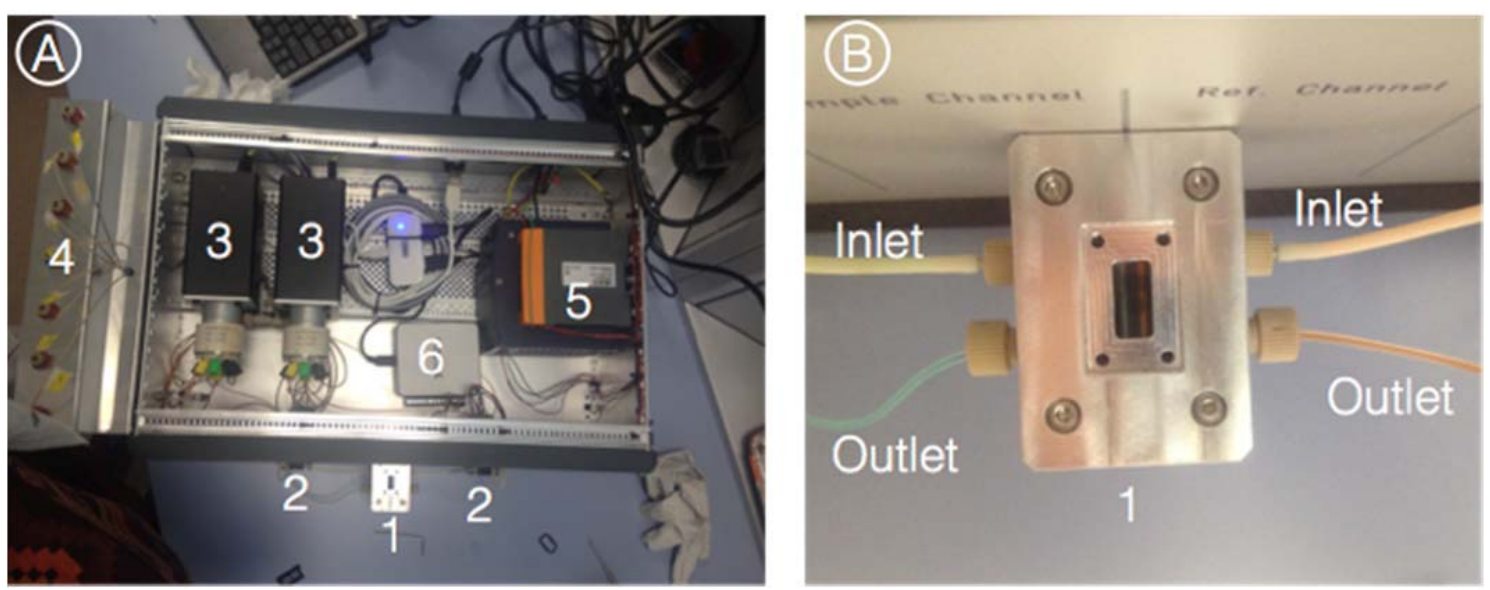

Figure 58: A. Fluidic system. 1-Fluidic chip holder. 2-Persitaltic pumps. 3-Rotatory valves. 4- Reservoires. 5-Feeding source. 6-Connexion to the computer. B. Fluidic hoder showing the two independent fluidic channels. Each of them with one inlet and one outlet.

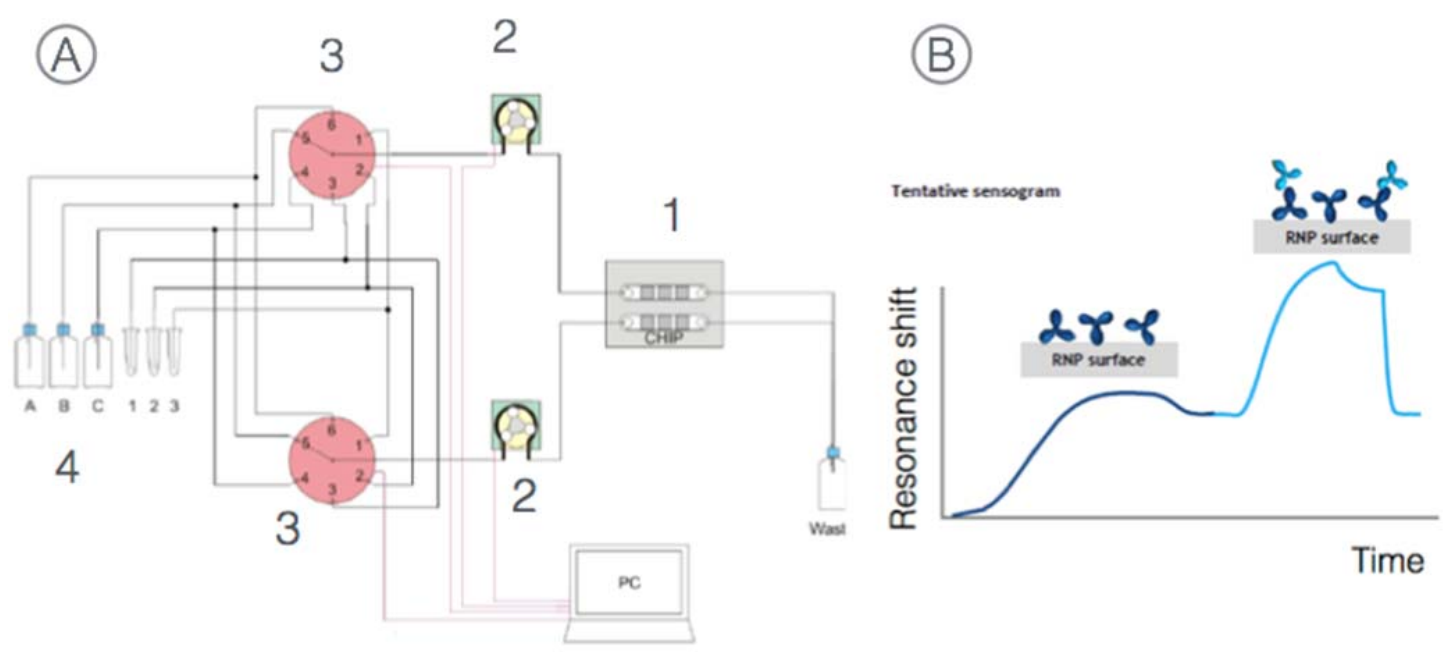

Figure 59: Fluidic scheme of the fluidic system. B Typical response of a continuous flow real-time experiment.

The maximum flow rate of the peristaltic pumps is $75 \mu \mathrm{L} / \mathrm{min}$. The control of the system with a standard PC, enables the user to implement simple protocols, for continuous flow, selecting times, flow rates and reservoir fluids, and repeat this, time after time. For the interrogation of the BICELLs, the fluidic chip holder was adapted to the optical set-up system described in the former subchapter. 
In UPM there was not a simultaneous read-out system, and each of the eight BICELLs, was independently interrogated with one single fiber bundle as describe in 6.3 subchapter.

The data processing for continuous flow experiments was performed with a specific software developed ad-hoc by the Centro de Electrónica Industrial (CEI) from UPM, by monitoring the resonant mode along the time. [Velasco 2016].

\subsection{Chemical Detection Unit (CDU)}

The chemical detection unit integrates the fluidic system and also an optical reader head for simultaneous interrogation of all the BICELLs. Thus the purpose of the fabrication of the CDU was to be able to measure simultaneously the different BICELLs integrated in a chip, in real time experiments.

For the operationally of the chemical detection unit framed in Enviguards Project, it was decided to fabricate chips with twelve BICELLs. This configuration allows the detection of three different chemical pollutants by measuring each of them three different times simultaneously and thus providing the results with statistics support. Also reference BICELLs could be measured three times. Figure 60. 


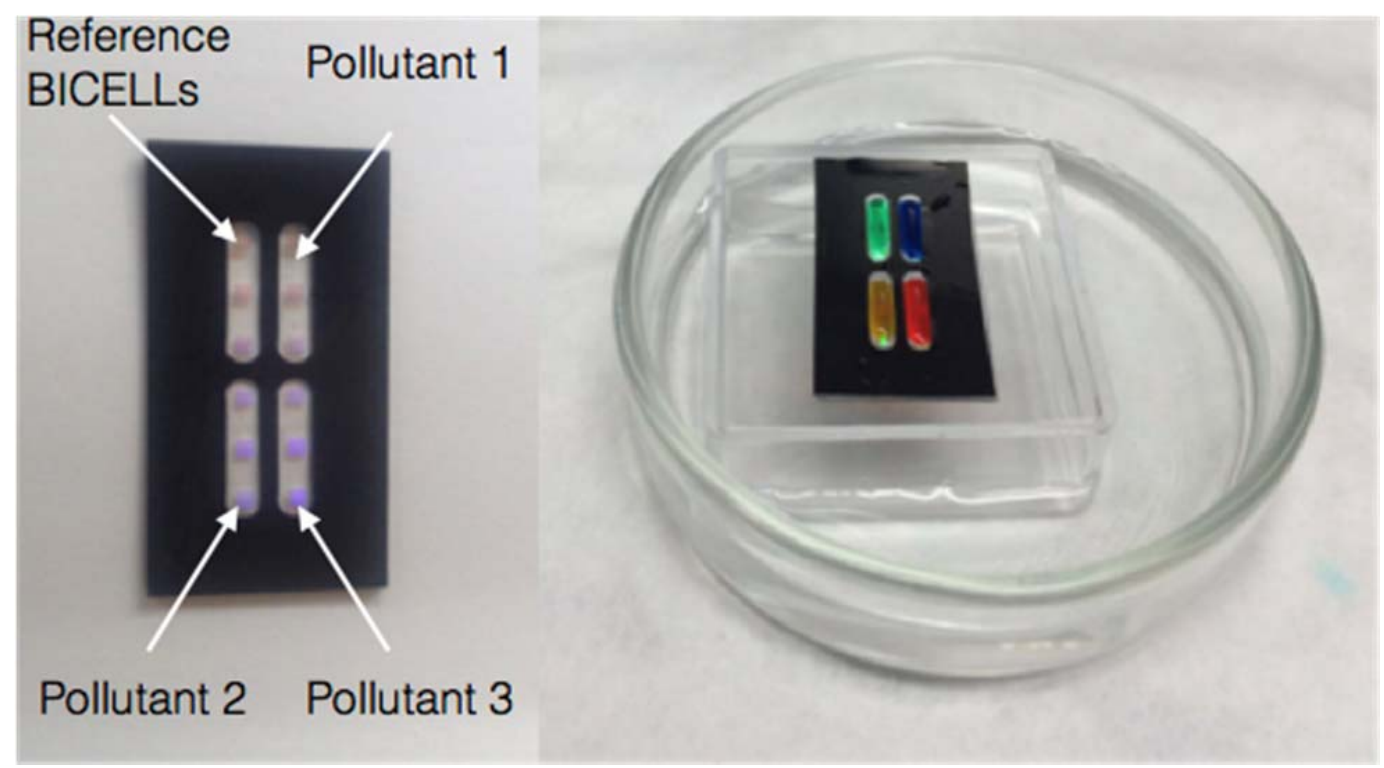

Figure 60: On the left. Chip with twelve BICELLS of R-NPs covered with a vinyl adhesive. On the right. Example of independet biofunctionalization of the different BICELLS.

Figure 60 represents on the left, a chip with twelve BICELLs, grouped in groups of three with an adhesive of vinyl. In the picture of the right it can be seen an example of the effectiveness of this vinyl adhesive to separate BICELLs for different bioreceptor immobilization purposes. Unfortunately, no experiments with this vinyl adhesive were accomplished in this thesis, it represents an example of how independent biofunctionalization could be performed without cross reactivity among BICELLs. This is part of the future prospects of this thesis.

CDU was assembled in the laboratory of the GOPB by integrating the different components: New fluidic system and new optical head.

The fluidic system was fabricated by connecting the twelve BICELLs in one single fluidic channel, with one inlet and one outlet. It was changed dimension of the chipholder in order to hold the twelve BICELLs integrated into the chip. Fluidic system incorporated a sample loop, so the sample could be mixed with the competitive analyte, before reaching the BICELLs, in order to develop competitive immunoassay. 
The optical head with twelve optics fibers was coupled to the chip holder, for the simultaneous interrogation of the twelve BICELLs. Twelve fibers were collected in a bundle and conected to the light soruce and spectrometer, everything integrated in the CDU housing. (Figure 61).

CDU remained in the GOPB laboratory a few days, thus, the time to develop experiment with it was so constrained.
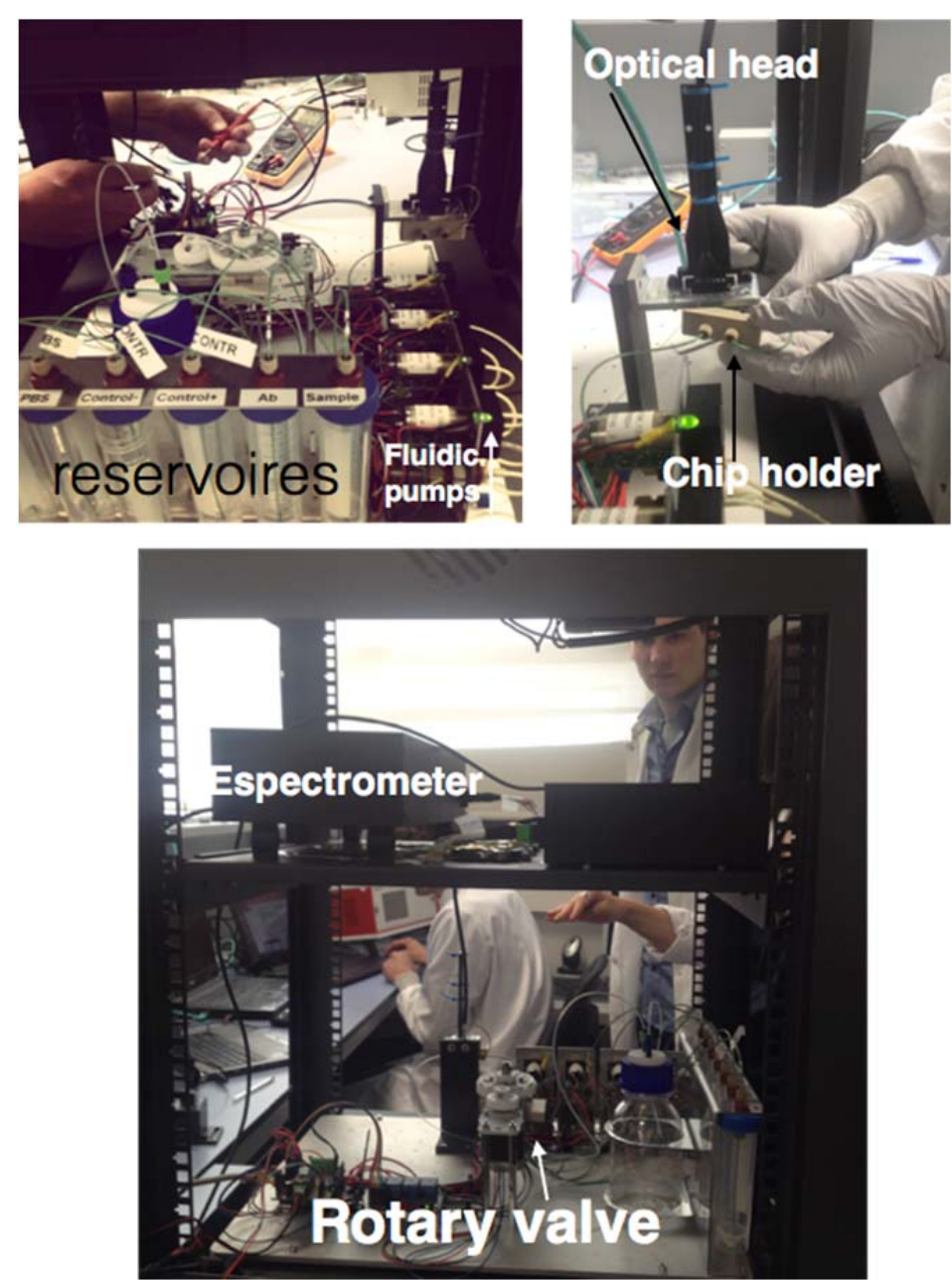

Figure 61: Different photos of the CDU integration in the UPM. 


\subsection{Summary}

It has been described the read-out systems used in this work. FT-IR and the optical set-up system mounted in the laboratory of the GOPB. Also it has been explained how the R-NPs were located in a specific holder ad-hoc or in the flow cell from fluidic system, for the different experimental methods: static or continuous flow measurements.

Also is has been briefly described the simultaneous read-out system. 
Chapter 6: Optical Interrogation of R-NPs 


\section{R-NPs AS BIOCHEMICAL SENSORS}

In this chapter it is studied the R-NPs performance as biochemical sensors. The methodology of study is based on bulk sensing experiments by observing the experimental optical response of the R-NPs as a function of different RI solutions.

\subsection{Bulk sensing performance of $\mathrm{SiO}_{2}$-NPs versus $\mathrm{R}-\mathrm{NPs}$}

The first experiment was focused on the experimental motivation of the use of R-NPs as biochemical sensors. As already explained in this thesis, the advantage of using R-NPs is not only the light confinement due to its periodic nanopillars structure, but also the increase of reflectivity and the formation of a marked resonant mode due to the Bragg reflectors and the central cavity. For this reason it was necessary to characterize and compare the yield of two different sorts of nano-pillars (NPs). On one hand simple silicon oxide nanopillars ( $\mathrm{SiO}_{2}$-NPs) with no reflectors, and on the other hand the R-NPs. [Cornago, 2016]. For the experiment purpose, firstly, it was measured the reflection spectra as a function of wavenumber of both types of NPs arrays fabricated with the same pitch $(300 \mathrm{~nm}$ for this case), and for different RI fluids, Di-water ( $n=1.33$ ), 20\% ethanol in water $(n=1.336)$ and pure ethanol $(n=1.36)$. The measurements were performed by vertical interrogation with the FT-IR (see chapter 6.1) [Holgado, 2010], [Laguna, 2014b]. $\mathrm{SiO}_{2}$-NPs arrays were fabricated over a transparent glass substrate so R-NPs arrays could be independently interrogated from the backside with the pillars facing down, in the holder fabricated ad-hoc. As explained in the fabrication chapter, R-NPs were fabricated over a transparent quartz substrate for the same purpose. Notice that for the fabrication process of substrates with the $\mathrm{SiO}_{2} / \mathrm{Si}_{3} \mathrm{~N}_{4}$ multilayer stack the average vertical etch rate was $16.1 \mathrm{~nm} / \mathrm{min}$. For $\mathrm{SiO}_{2}$, using the same RIE recipe, the etching rate was $17 \mathrm{~nm} / \mathrm{min}$. Thanks to this small difference; the height of the R-NPs was very similar to the $\mathrm{SiO}_{2}$-NPs. 

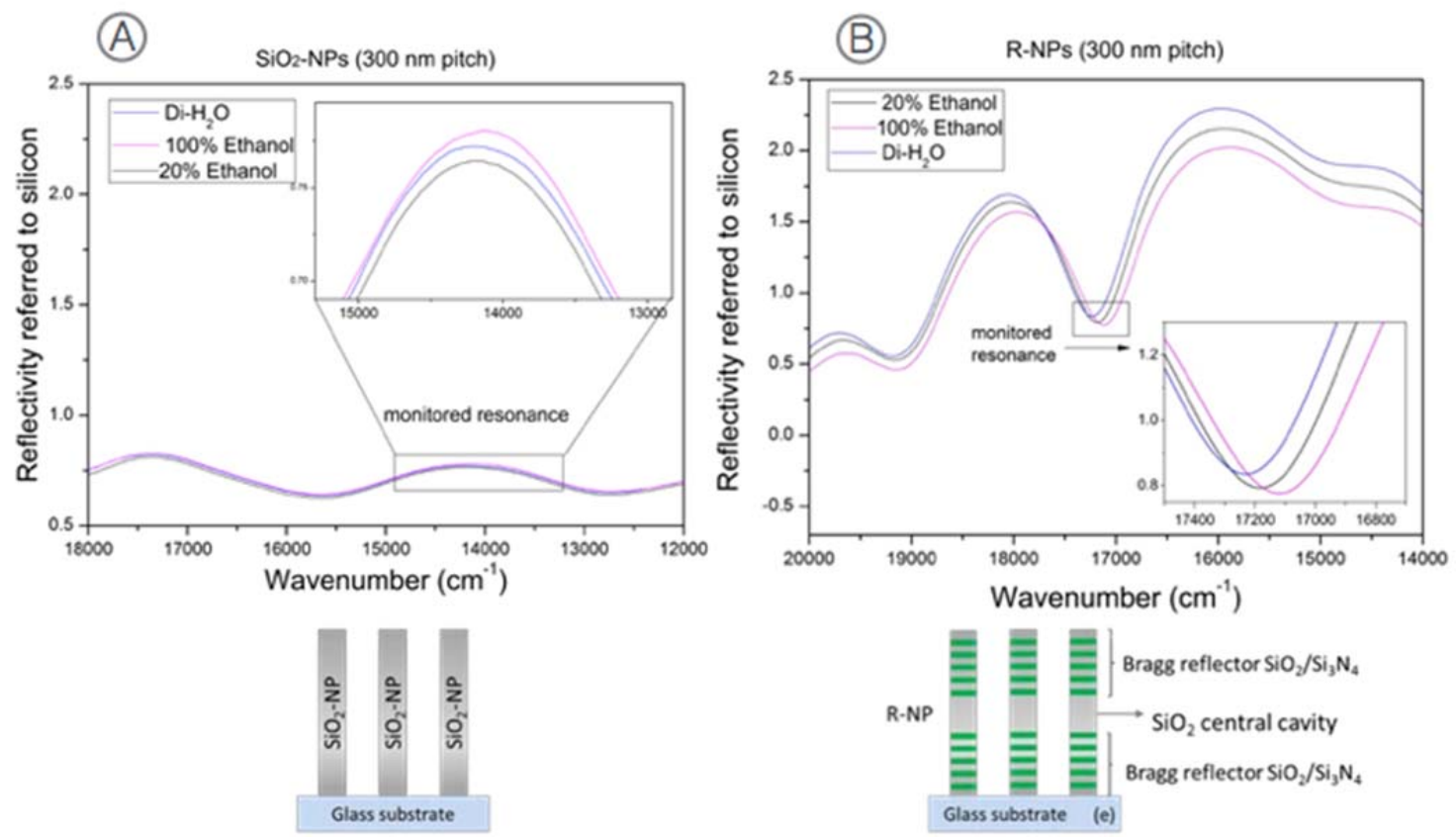

Figure 62: Resonant modes of NPs immersed in fluid: $\mathrm{A}$. $\mathrm{SiO}_{2}-\mathrm{NPs}$ with $300 \mathrm{~nm}$ pitch. B. R-NPs with $300 \mathrm{~nm}$ pitch.

Because the R-NPs arrays are also mainly based on $\mathrm{SiO}_{2}$, it was found that there were not significant visual differences in their wettability, and therefore it was not observed any difference during the infiltration of the different RI fluids between both types of arrays. However, it was observed that the $\mathrm{SiO}_{2}$-NPs reflectivity amplitude signal was much lower than for the case of the R-NPs. (Figure 62).

In addition, it can be observed multiple small resonances for $\mathrm{SiO}_{2}$-NPs and a sharper resonant mode for the case of the R-NPs. The $\mathrm{SiO}_{2}$-NPs spectra has several dips and peaks than can be monitored, whereas the R-NPs has a single resonance mode, which can be named resonant dip when it is interrogated by reflectometry.

It is desirable to have a uniform mechanism for comparing the bulk sensing results, but to date there exist a degree of variation as to how the sensing performance is quantified. [White, 2008]. For the comparison of the sensitivity of

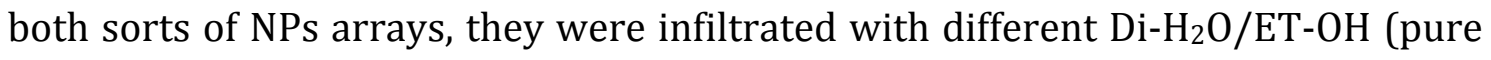
water/ethanol) fraction solutions and subsequently optically interrogated in order to monitor the shift of the narrowest resonance mode position according to RI 
variation. Every solution was measured seven times for the standard uncertainty calculation. The shift in $\mathrm{cm}^{-1}$ can be expressed as a function of RIU (Refractive Index Units) (Figure 63). The ethanol content in $\mathrm{Di}-\mathrm{H}_{2} \mathrm{O}(\mathrm{n}=1.33)$ of the solutions was: $10(n=1.333), 20(n=1.336)$ and 100\% $(n=1.36)$ (vol.) and deionized water was employed as the reference. This is called a bulk sensing experiment and this type of experiment was accomplished in this thesis for the different R-NPs samples comparison.

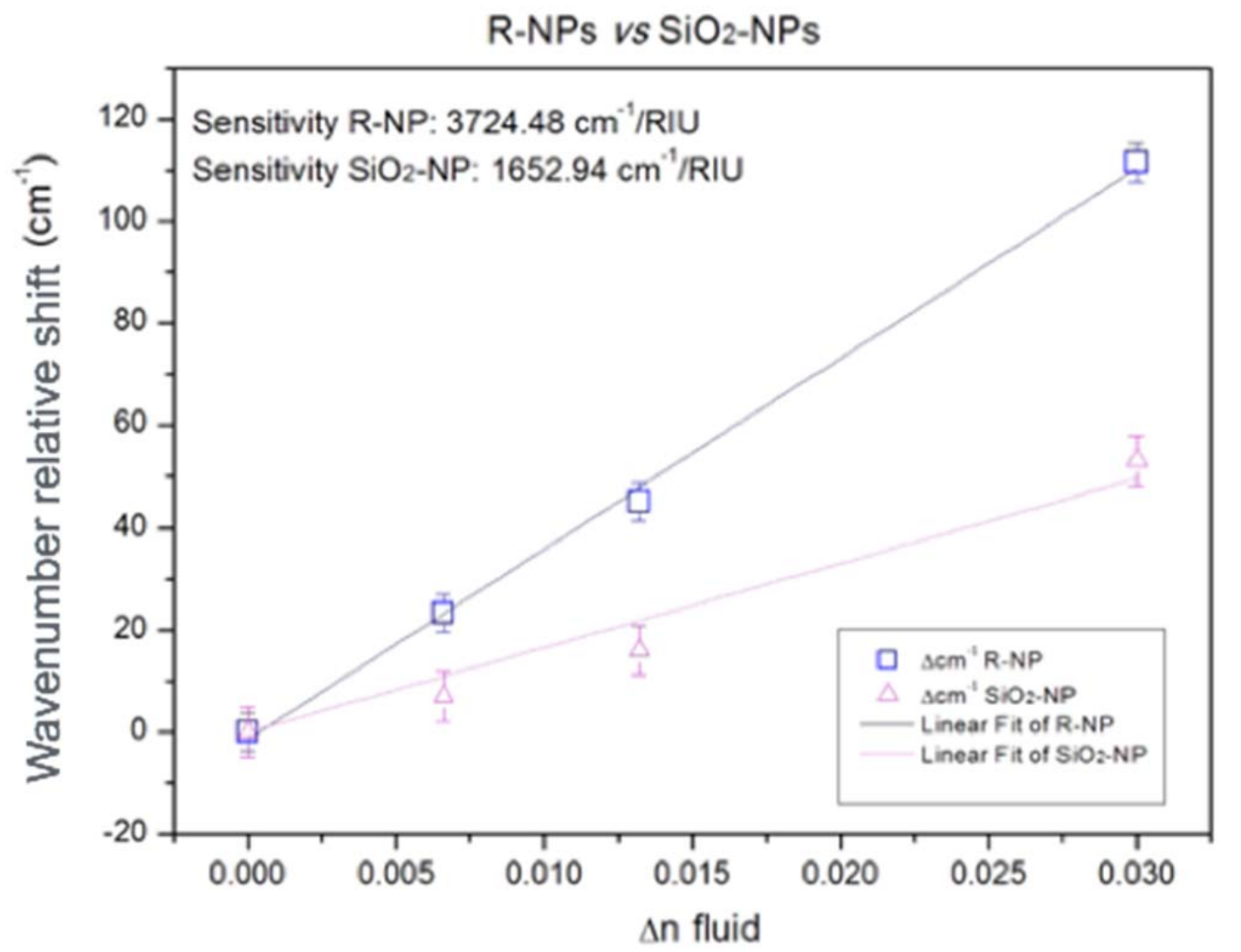

Figure 63: Resonance wavenumber position as a function of refractive index units (RIU). The blue squares and pink triangles correspond to R-NPs and $\mathrm{SiO}_{2}$-NPs response respectively.

As a result, it was drawn that sensitivity, calculated as the wavenumber shift divided by the refractive index variation $\left(\mathrm{cm}^{-1} / \mathrm{RIU}\right)$, reached a value of $3724.48 \mathrm{~cm}^{-1} /$ RIU for the R-NPs in contrast to the sensitivity obtained for the $\mathrm{SiO}_{2}$-NPs that reached a considerable lower value of $1652.94 \mathrm{~cm}^{-1} / \mathrm{RIU}$. Both bulk sensing responses were obtained exactly under the same conditions: same optical interrogation system, same ethanol-water solutions, humidity and room temperature at $20^{\circ} \mathrm{C}$. The experimental uncertainty $(U)$, was obtained considering 
the resolution of the system employed of $4 \mathrm{~cm}^{-1}$ for a coverage factor of $\mathrm{k}=3$. Uncertainty was $4.9 \mathrm{~cm}^{-1}$ for the $\mathrm{SiO}_{2}$-NPs, and $3.7 \mathrm{~cm}^{-1}$ for the R-NPs, which resulted in a limit of detection ( $L o D$ ) of $2.9 \times 10^{-3}$ RIU and $9.9 \times 10^{-4}$ RIU, respectively. However, if there was only considered as uncertainty factor, the maximum resolution of the measuring system $\left(1 \mathrm{~cm}^{-1}\right)$, the LoD would be $2.6 \times 10^{-4}$ RIU for R-NPs with $300 \mathrm{~nm}$ of lattice parameter.

Thus, it was determined for the first time the benefits of using R-NPs in comparison with other nanostructures such as $\mathrm{SiO}_{2}$-NPs arrays, in terms of bulk sensitivity. In fact, it was demonstrated that chemical bulk sensitivity of R-NPs arrays doubles the $\mathrm{SiO}_{2}$-NPs and the LoD is also improved by a factor of 4 , using the FT-IR for the optical interrogation, demonstrating their higher performance and suitability to be used as biochemical transducers. [Cornago, 2016]. This work was a preliminary test of the performance of the R-NPs, however the effect of the lattice parameter or the number of Bragg reflector pairs were not considered in detail.

\subsection{Influence of the number of the Bragg Reflectors}

In chapter 4.1 it has been studied the effect in evanescent field of the different lattice parameters, claiming that the closer the nanopillars are, the greater it is the light confinement and therefore the reflectivity of the nanopillars. From section 4.2.3 it is drawn that the most sensitive structures are those composed by the greatest number of pair reflectors. Even though the afore-mentioned follows theoretical assumptions based in an ideal fabrication and design of the R-NPs, however, the experimental results do not always match $100 \%$ with the predicted in the simulations.

In order to be able to compare experimental optical response of R-NPs with different number of pair reflectors and lattice parameters or pitch, there were characterized three samples with 18 reflector pairs, each of them with a different lattice parameter $(300,400,600 \mathrm{~nm})$. In addition three samples with those lattice 
parameters with 10 pair of Bragg reflectors were also characterized. A SEM image of the different R-NPs configurations measured is showed in figure 64 .

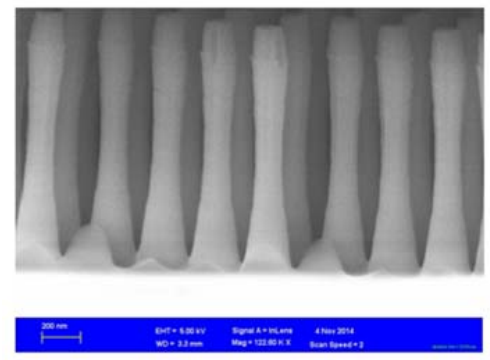

18 BR-300nm

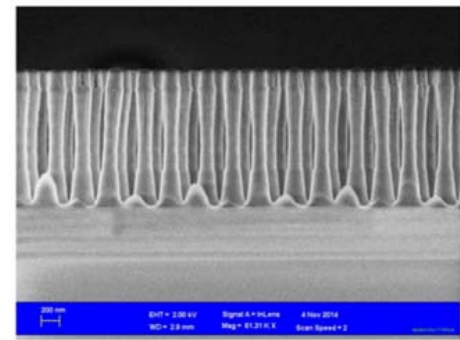

10 BR-300nm

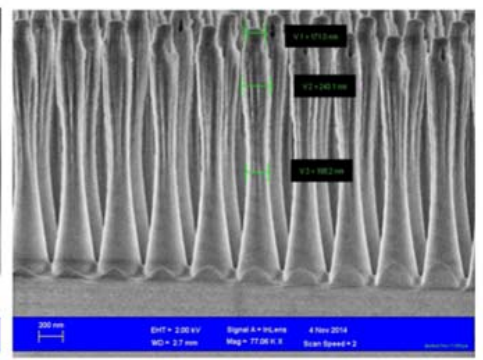

18 BR-400nm

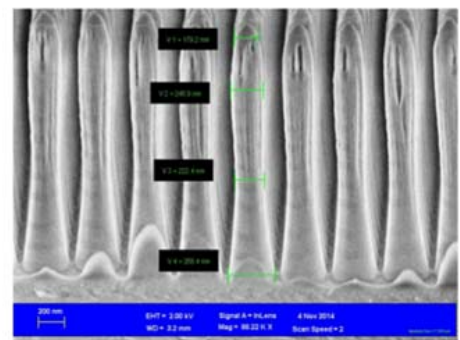

10 BR-400nm

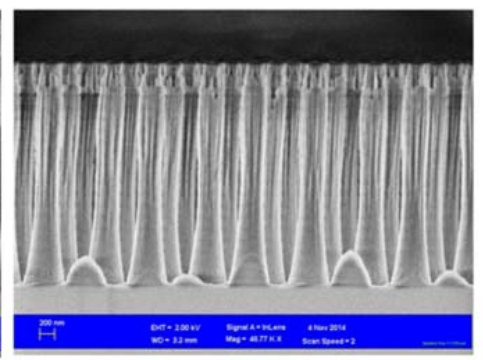

18 BR-600

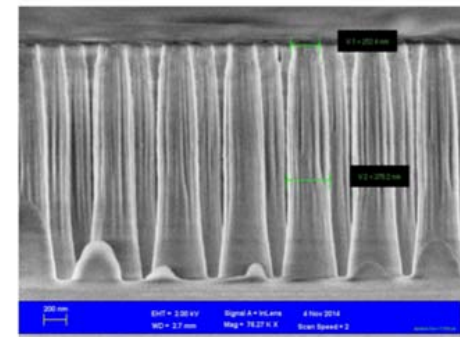

10 BR-600

Figure 64: SEM image of the R-NPs with ten and eighteen Bragg Refectors (BR) and different lattice parameter.

Next figure represents the optical response of the R-NPs immersed in water as a function of the wavelength (nm), with different pitch and Bragg reflectors pairs.
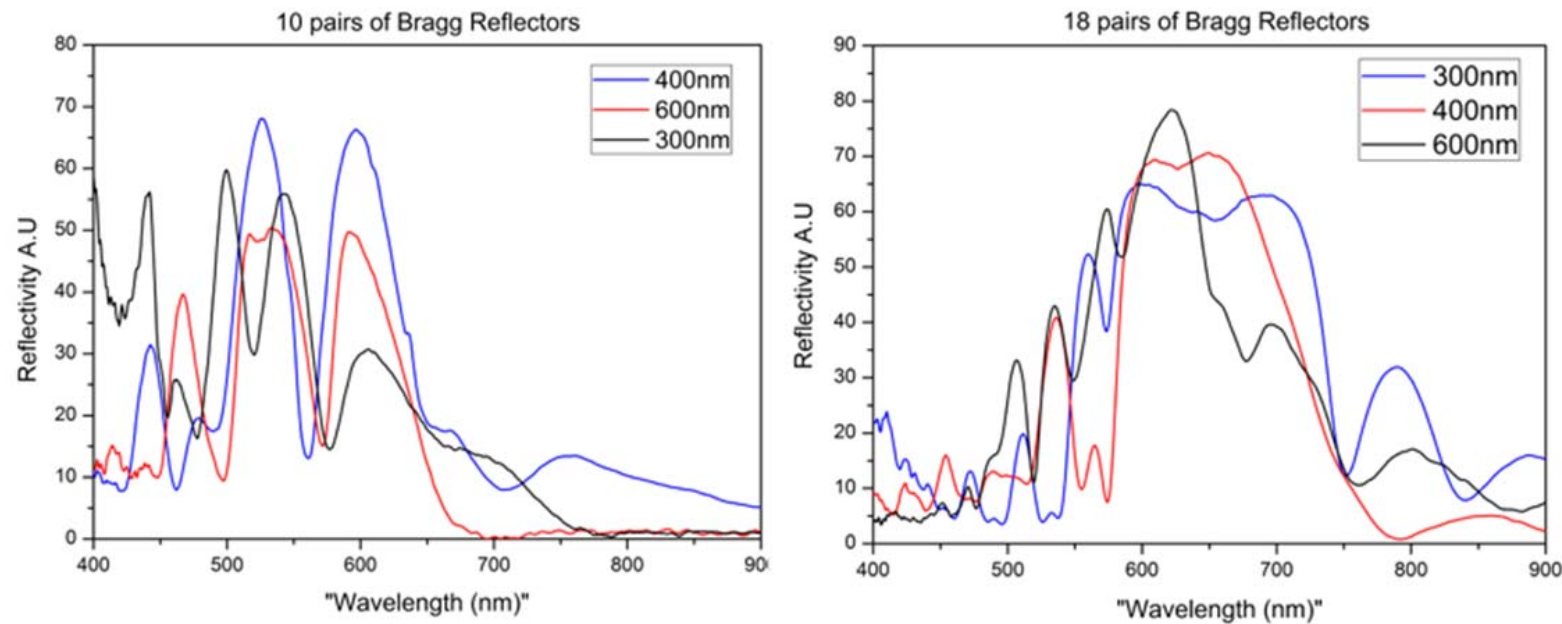

Figure 65: Optical response of R-NPs with ten and eighteen BR pairs and different lattice parameter (300, 400 and 600$)$. 
As it could be expected, the absolute reflectivity is higher for samples with 18 Bragg reflectors. However this enhancement of the reflectivity is not very acute. What it is remarkable is the formation of a much more clear resonance mode in samples with 10 pairs of Bragg reflectors; especially in the sample with a pitch of $400 \mathrm{~nm}$. The resonant mode in samples with 18 pairs of Bragg reflectors do not show a clear resonance mode in the center of a gap, suggesting that probably the fabrication process did not success and that it could be, that the etching process did not fully reached the central cavity, leaving an incomplete formation of the photonic gap and a resonant mode off center. Thus experiments continued by using samples with 10 pairs of Bragg reflectors.

\subsection{Effect of the lattice parameter on R-NPs with 10 pairs of Bragg Reflectors}

It is presented the study of the effect of the lattice parameter (which will be also referred as pitch) of the R-NPs in terms of bulk sensitivity. The position of the resonant mode varies when the lattice parameter change, as can be seen in Figure 66. For a pitch of $300 \mathrm{~nm}$ the resonance is centered on $17250 \mathrm{~cm}^{-1}$ (figure 62. B), whereas for 400 and $600 \mathrm{~nm}$ it is centered around 17900 and $17500 \mathrm{~cm}^{-1}$, respectively.

Samples of $300 \mathrm{~nm}$ pitch were studied in the chapter 7.1. In order to study also samples of 400 and $600 \mathrm{~nm}$ pitch, each of them were placed in the holder and it was introduced first $\mathrm{Di}-\mathrm{H}_{2} \mathrm{O}$ and then it was introduced ethanol for the optical response acquisition comparison. 

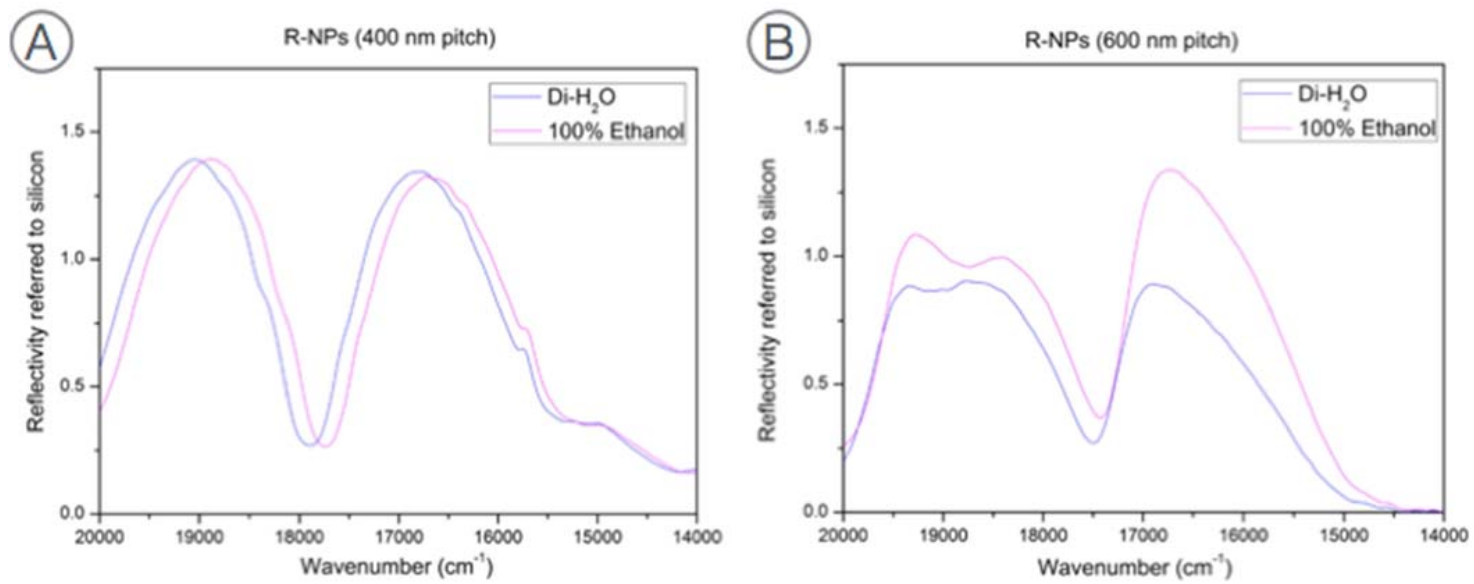

Figure 66: Reflectivity of R-NPs immersed in ethanol and water. A. pitch of $400 \mathrm{~nm}$. B. Pitch of $600 \mathrm{~nm}$.

It was then, obtained the bulk sensitivity for three different values of pitch of R-NPs (300, 400 and $600 \mathrm{~nm})$. The sensitivity was calculated considering the linear range between the position of the resonance when measuring $\mathrm{Di}-\mathrm{H}_{2} \mathrm{O}$ and the position of the resonance for the pure ethanol measurement. For a pitch of $400 \mathrm{~nm}$ the obtained sensitivity was of $4068.39 \mathrm{~cm}^{-1} / \mathrm{RIU}$, whereas for $600 \mathrm{~nm}$ this value is of $2303.33 \mathrm{~cm}^{-1} /$ RIU. Theoretically, lower values of pitch result in a better value of sensitivity. This is clear in the case of $600 \mathrm{~nm}$; however, values for 300 (3724.48 $\left.\mathrm{cm}^{-1} / \mathrm{RIU}\right)$ and $400 \mathrm{~nm}\left(4068.39 \mathrm{~cm}^{-1} / \mathrm{RIU}\right)$ are much closer and do not follow this theoretical rule. This is probably due to the different values of diameter and height for the two different pitch arrays. Considering the samples with $400 \mathrm{~nm}$ pitch revealed the maximum sensitivity among the other pitches tested, it was carried out a much more detailed bulk sensing experiment over R-NPs of $400 \mathrm{~nm}$ pitch and 10 pair of Bragg reflectors in order to observe their maximum limit of the detection for biochemical sensing.

\subsection{Static Bulk sensing experiment on R-NPs as biochemical sensors}

R-NPs with $400 \mathrm{~nm}$ of lattice parameter showed a greater sensitivity. Thus, a more complete study was developed on the performance indicators of R-NPs such as sensitivity and limit of detection. For that reason it was performed a bulk sensing 
experiment, measuring fluids with increasing RI values. The results of this work were published in a scientific journal. [Hernandez, 2015].

For this experiment it was used a $14 \mathrm{~mm} \times 20 \mathrm{~mm}$ glass substrate, including two rows and four arrays of RNPs. Each RNP perpendicularly arranged to the substrate, consists of 10 pairs of $\mathrm{Si}_{3} \mathrm{~N}_{4} / \mathrm{SiO}_{2}$ reflectors. The thickness of each layer, determined by the starting multistack was $110 \mathrm{~nm}$ for $\mathrm{SiO}_{2}$, and $80 \mathrm{~nm}$ for $\mathrm{Si}_{3} \mathrm{~N}_{4}$, and $210 \mathrm{~nm}$ for the $\mathrm{SiO}_{2}$ central cavity. [Cornago, 2016]. Each RNP height was around $2000 \mathrm{~nm}$, the diameter was in the order of $200 \mathrm{~nm}$ and the pitch among RNPs was $400 \mathrm{~nm}$.

For the optical response characterization of each array of RNPs, it was used a highresolution FT-IR Spectrometer because the optical set-up based on the Mightex spectrometer (described in chapter 6.3) was not available by that time. The setup allows obtaining a separate spectral response for each array that collects the reflection from the backside of the substrate. It was employed an optical objective with a magnification of $4 \times$ at normal incidence, and the spectra were carried out with a resolution of $1 \mathrm{~cm}^{-1}$ and 200 scans, as previously reported [Sanza, 2011]. Since the dimensions of the arrays were $1 \mathrm{~mm} \times 1 \mathrm{~mm}$, it was adjusted the beam spot size to fit this dimension by the Hyperion microscope edge apertures attached to the spectrometer.

The chip was placed on the holder (characterization) with the arrays of R-NPs facing down in order to be in contact with the liquid. Reflectivity measurements from the backside were carried out for the different fluids participating in the bulk sensing experiment. The Bragg reflectors and the vertical cavity of each R-NPs array produced a resonant dip that can be clearly observed in the reflectivity profile. (Figure 67).

In figure 67 it is presented the transducing signal for different situations. The green line $(A)$ is the optical response of the multilayers stack without the fabrication of the R-NPs. The resonant mode position is observed at $15600 \mathrm{~cm}^{-1}$, with a relatively good Q-factor. After fabricating the pillars, the refractive index 
(RI) distribution of the film multistack changed from a stack of thin layers of $\mathrm{Si}_{3} \mathrm{~N}_{4} / \mathrm{SiO}_{2}$ to a mixture of these layers and a fluid. This resulted in a change in the effective RI of the central cavity, and thus in a shift in the position of the resonance. In fact, the resonant mode shifted from $15600 \mathrm{~cm}^{-1}$ approximately to a higher wavenumber located at $20070 \mathrm{~cm}^{-1}$. It is represented through the black line (B). Then, when it was introduced $\mathrm{Di}-\mathrm{H}_{2} \mathrm{O}$ among R-NPs the optical response shifted again (blue line, C) but to a smaller wavenumber (higher wavelength) of $17831 \mathrm{~cm}^{-1}$ because the RI of the surrounding media varied from 1 (RI of the air) to 1.33 (RI of the water). It was ready to detect different fractions of ethanol. The displacement found for $40 \%$ of ethanol in $\mathrm{DI}-\mathrm{H}_{2} \mathrm{O}$ was $135 \mathrm{~cm}^{-1}$, which corresponds to a position of the resonance dip at $17696 \mathrm{~cm}^{-1}$ (pink line, D).

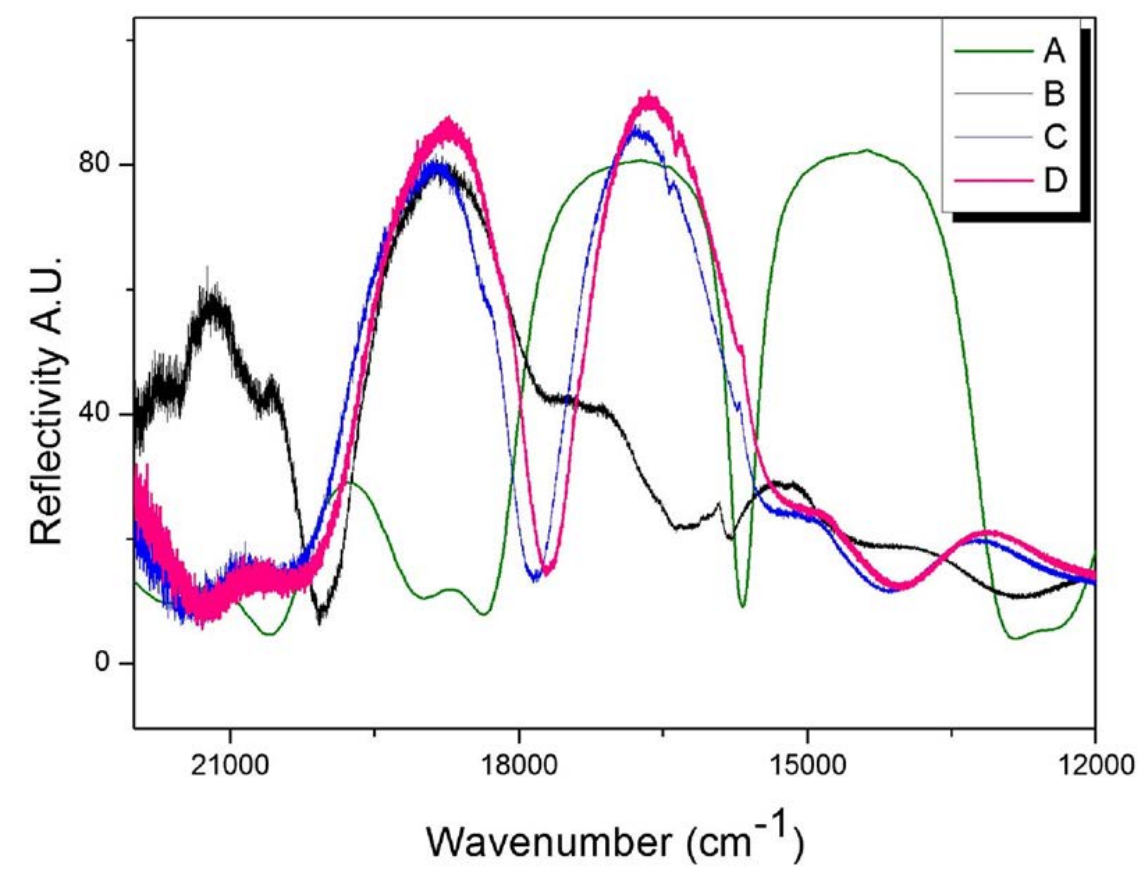

Figure 67: Transducing signal for different situations. A. Bulk multistack (green line). B. RNPs arrays response without liquid infiltration (black line). C. R-NPs array with water infiltration (Blue line). D. R-NPs array with $40 \%$ ethanol in water infiltration (Pink line).

For the bulk sensing experiment, it was monitored the wavenumber shift $\left(\mathrm{cm}^{-1}\right)$ of the resonance dip as a function of the RI of the liquid infiltrated. (Figure 68). For 
this purpose, there were used mixtures that consisted of $0 \%, 5 \%, 10 \%, 15 \%, 20 \%$ and $40 \%$ of ethanol, being the respective RI: $1.33,1.3315,1.333,1.3345,1.336$, 1.342 .

These indexes $(n)$ were calculated as follows:

$$
\left.n 1=\left(\frac{V \text { ethanol }}{V \text { total }} * n \text { ethanol }+\frac{V \text { agua }}{V \text { total }} * n \mathrm{H}_{2} \mathrm{O}\right)-n \mathrm{H}_{2} \mathrm{O} \text { (Equation } 19\right)
$$

The concentrations used were only up to $40 \%$ of ethanol so as to obtain the maximum sensitivity from the linear range. Each fluid was introduced between R-NPs and the base holder to take the reflectivity measurement, and then R-NPs and holder were rinsed with $\mathrm{Di}-\mathrm{H}_{2} \mathrm{O}$ and dried with clean air, for the next fluid measurement. In order to obtain the experimental uncertainty, the measurement of each liquid infiltration into RNPs array was repeated seven times.

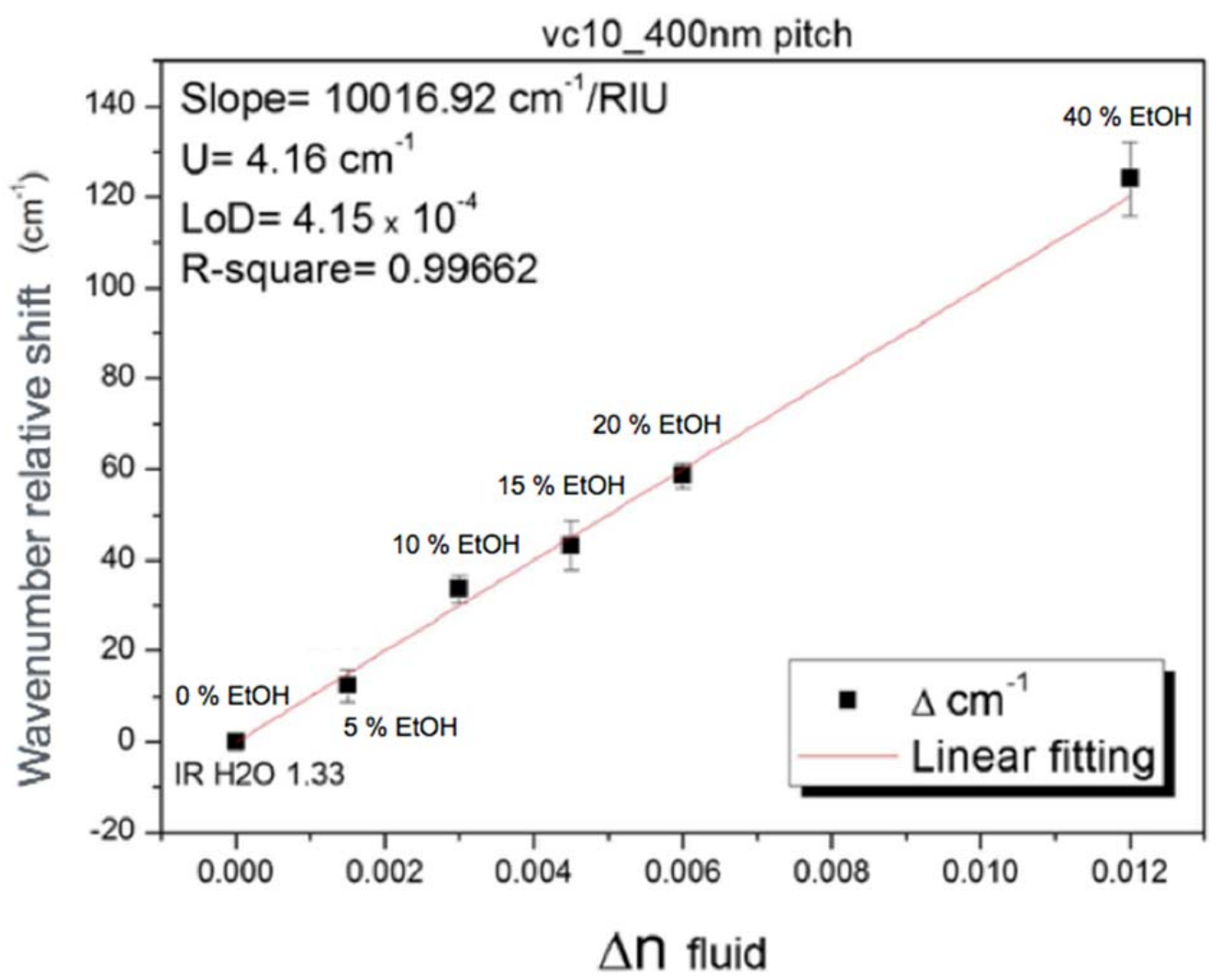

Figure 68: Shift of wavenumber as a function of the RI change. 
The points relative to different mixtures are fitted into a linear response with a correlation of 0.997 between the resonance wavenumber dip position and the liquid RI infiltrated in Refractive Index Units (RIUs). This slope can be considered as the bulk sensitivity sensitivity $m$ of the R-NPs array transducer, resulting in a value of $10017 \mathrm{~cm}^{-1} / \mathrm{RIU},(326 \mathrm{~nm} / \mathrm{RIU})$.

In order to estimate the Limit of Detection (LoD), it should be bearded in mind the expanded uncertainty $(U)$ during the optical integration process. This expanded uncertainty was calculated with a coverage factor of 3 following the International Union of Pure and Applied Chemistry (IUPAC) recommendation and according to the equations from the Guide to the Expression of Uncertainty in Measurements [GUM] as already explained in this thesis. (Equations 8 and 9).

The experimental LoD was calculated as the quotient between $U$ and $m$ $(L o D=U / m)$. Although it was found some dispersion in the value of $U$ for the different fractions of ethanol, it was estimated the LoD by considering the average of $U$ for all the measurements, resulting in a value of $4.5 \times 10^{-4}$ RIU. This is an estimation of the minimum RI variation that it was possible to detect with the above-mentioned experimental conditions.

The sensitivity of the R-NPs, was calculated in $\mathrm{cm}^{-1} / \mathrm{RIU}$, because FT-IR, by default measures the reflectivity signal as a function of the wavenumber $\left(\mathrm{cm}^{-1}\right)$ and thus, resolution is also given in $\mathrm{cm}^{-1}$. Even though, the system allows changing the abscissa exe from the reflectivity signal to wavelength units (nm). Thus, the same bulk sensing experiment was calculated as function of wavelength. The OPUS software made conversion numerically. 

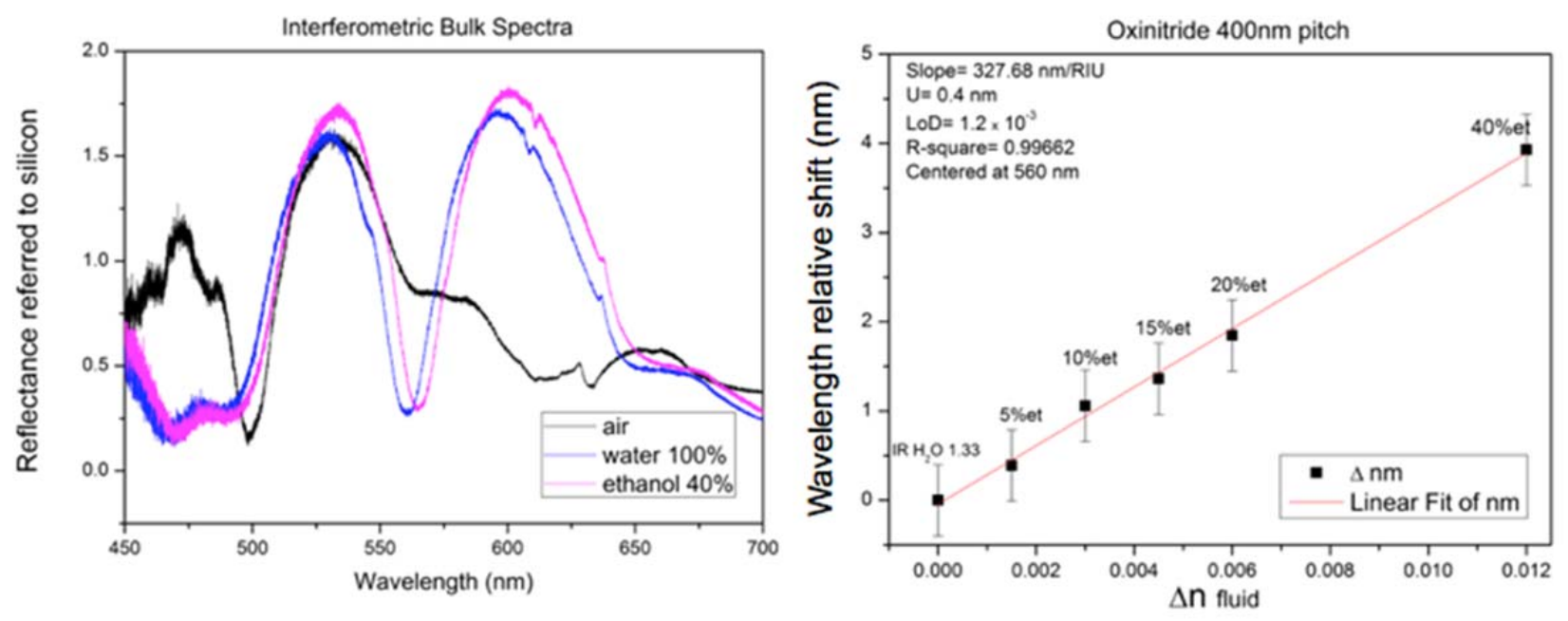

Figure 69: On the left. Transducing signal as function of the wavelenght for different situations of the R-NPs. On the right. Shift of wavelength as a function of the RI change.

The optical response of the R-NPs measured in $\mathrm{Di}-\mathrm{H}_{2} \mathrm{O}$ shows a resonant mode centered at $17831 \mathrm{~cm}^{-1}$. This corresponds to $560 \mathrm{~nm}$ approximately. For $40 \%$ of ethanol the resonance was centered at $17969 \mathrm{~cm}^{-1}$, corresponding to $563.9 \mathrm{~nm}$. The slope was then, $327.68 \mathrm{~nm} /$ RIU. The uncertainty was slightly increased, since the resolution had to be calculated manually according to the spectral range position of the resonance being monitored. This explained that the LoD in this case was one order of magnitude lower $\left(1.2 \times 10^{-3}\right.$ RIU), decreasing the sensing performance. Even though the bulk sensitivity was still comparable to other optochemical systems reported.

Compared to previous works that used different biochemical sensors, it can be noticed that the R-NPs arrays equal or improve the sensitivity of other reported transducers. For example, the sensitivity reported for a transducer based on a photonic crystal microcavity [Chow, 2004] was $182 \mathrm{~nm} / \mathrm{RIU}$, and the sensitivity for the slot- waveguide-based ring resonator transducer [Barrios, 2007] was $212 \mathrm{~nm} / \mathrm{RIU}$.

It must be noticed that the LoD is given by the quotient of $U$ and $\mathrm{m}$. Thus, for the case of the slot-waveguide-based ring resonator transducer [Barrios, 2007], the reported LoD was $2.3 \times 10^{-5}$, considering $U$ as the maximum wavelength resolution 
of their system, (5 pm). Under those same optical read-out condition, the LoD achieved by the R-NPs, in this work would be $1.5 \times 10^{-5}$ RIU, which is a significant improvement.

\subsection{Redesign of $\mathrm{SI}_{3} \mathrm{~N}_{4} / \mathrm{SiO}_{2} \mathrm{R}-\mathrm{NPs}$}

The results showed until this point corresponds to of the R-NPs with ten Bragg reflector pairs of $\mathrm{Si}_{3} \mathrm{~N}_{4} / \mathrm{SiO}_{2}$ (80 and $110 \mathrm{~nm}$ respectively) and a central cavity of $\mathrm{SiO}_{2}(210 \mathrm{~nm})$. In order to improve the performance of the RNPs it was performed a redesign of the multistack, considering the real fabrication parameters: pitch $(400 \mathrm{~nm})$ and diameter $(200 \mathrm{~nm})$ experimentally studied. For that purpose, it was used the Marxwell-Garnet equation in order to calculate the effective refractive index of the material in function of the mentioned parameters.

With these indexes, it was recalculated the reflectors thickness to satisfy the lambdaquarter condition for both layers and the thickness of the cavity.

$$
n e f f 1 * t h 1=n e f f 2 * t h 2=\frac{\lambda G a p}{4}
$$

According to these thicknesses it must be selected as much reflector pairs as possible considering fabrication process limitations, which was in this case a maximum height of $2.5 \mu \mathrm{m}$ because of the RIE process.

Then, for a pitch of $400 \mathrm{~nm}$ and a diameter of $200 \mathrm{~nm}$ and considering a pure water medium, the value of the effective refractive index was 1.47 . The new composition of the multilayer was: ten pairs of $\mathrm{SiO}_{2} / \mathrm{Si}_{3} \mathrm{~N}_{4}(110 / 97 \mathrm{~nm})$ Bragg reflectors and a $\mathrm{SiO}_{2}$ central cavity of $200 \mathrm{~nm}$ thick. 

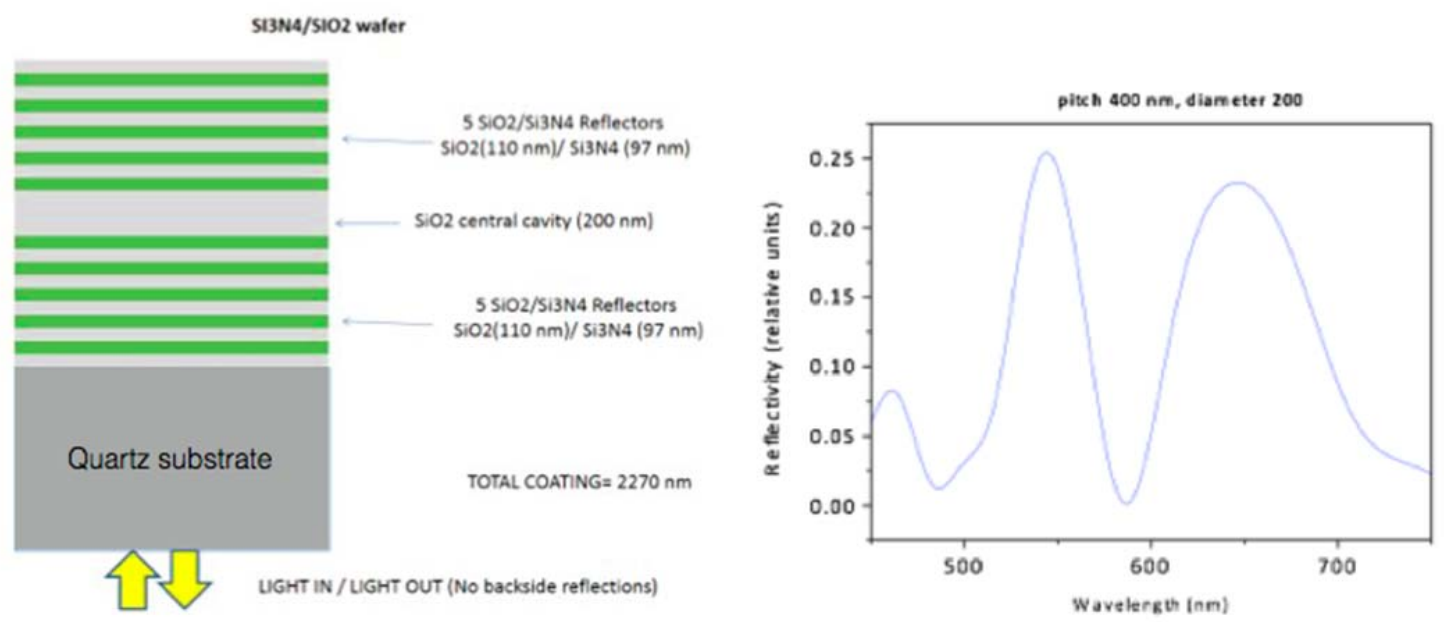

Figure 70: Schem of the redesigned multistack and the expected optical response.

With this new configuration the resonant mode for R-NPs of $400 \mathrm{~nm}$ of lattice parameter, immersed in water, was centered also at $560 \mathrm{~nm}$ approximately.

The same bulk sensing experiment described in the previous subchapter was accomplished for the redesigned R-NPs. For a proper comparison between the first R-NPs and the redesigned R-NPs, it was used the same holder, measuring system and the same water-ethanol fractions for the bulk sensing experiment.

In order to compare both transducers, it was studied the sensitivity which was increased by a factor of 1.8, from 327 to $591 \mathrm{~nm} /$ RIU. Since the uncertainty was practically the same, the LoD was improved from $1.2 \times 10^{-3}$ to $7.1 \times 10^{-4}$ Refractive Index Units. 

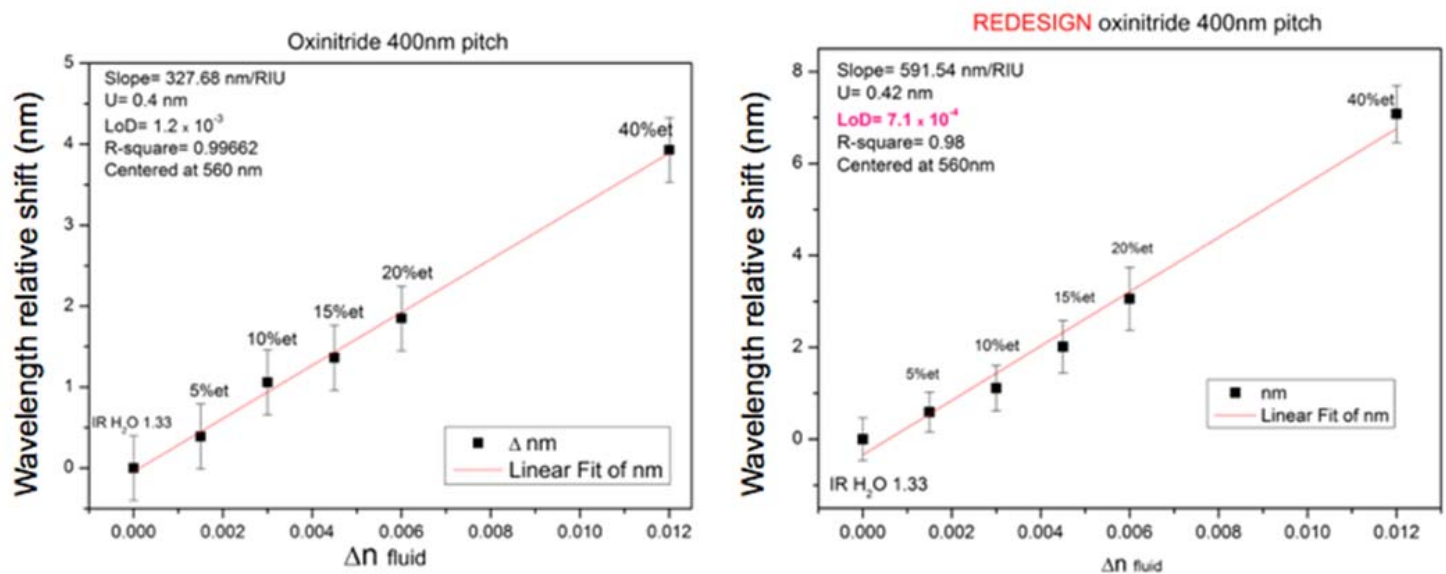

Figure 71: Comparison of the bulk sensing experiment for first R-NPs fabricated and redesigned $R-N P s$.

Table 2: Comparison data of the first R-NPs multistack and the Redesigned R-NPs multistack

\begin{tabular}{|c|c|c|}
\hline $\begin{array}{c}\text { Samples 400 } \mathrm{nm} \\
\text { pitch }\end{array}$ & First multistack & $\begin{array}{c}\text { Redesigned } \\
\text { multistack }\end{array}$ \\
\hline $\begin{array}{c}\text { Sensitivity } \\
\text { (nm/RIU) }\end{array}$ & 327.68 & 591.54 \\
\hline LoD & $1.2 \times 10^{-3}$ & $7.2 \times 10^{-4}$ \\
\hline
\end{tabular}

Within these results, it seemed difficult to still improve the performance without using other material with higher refractive index contrast, or considering higher nanopillars and more pairs of reflectors (limited by fabrication so far).

From this point onwards, all the R-NPs used in this work were the redesigned R-NPs above described.

\subsection{Multiplexing capacity of R-NPs and new lattice parameter}

One of the advantages of the R-NPs as optical label-free biosensor, is that they can be grouped into cells (BICELLs) and integrated in the same sensing chip. R-NPs arrays or BICELLs (when they are biofunctionalized) are thus, the individual 
sensing areas, which convert a one-test simple sensor into a multiplexed sensor, able to detect different biomolecules at a once. Therefore the homogeneity of the fabrication parameters along R-NPs arrays is a key point in order to achieve a multiplexed biosensor.

On the other hand, R-NPs samples with $500 \mathrm{~nm}$ of lattice parameter were fabricated in order to know if the homogeneity among cells was influenced or not by the manufactoring parameters.

Then, another bulk sensing experiment was performed over the two different samples tested: one with $400 \mathrm{~nm}$ pitch (resonance mode centered at 560nm) and other with $500 \mathrm{~nm}$ pitch (resonance mode centered at 580).

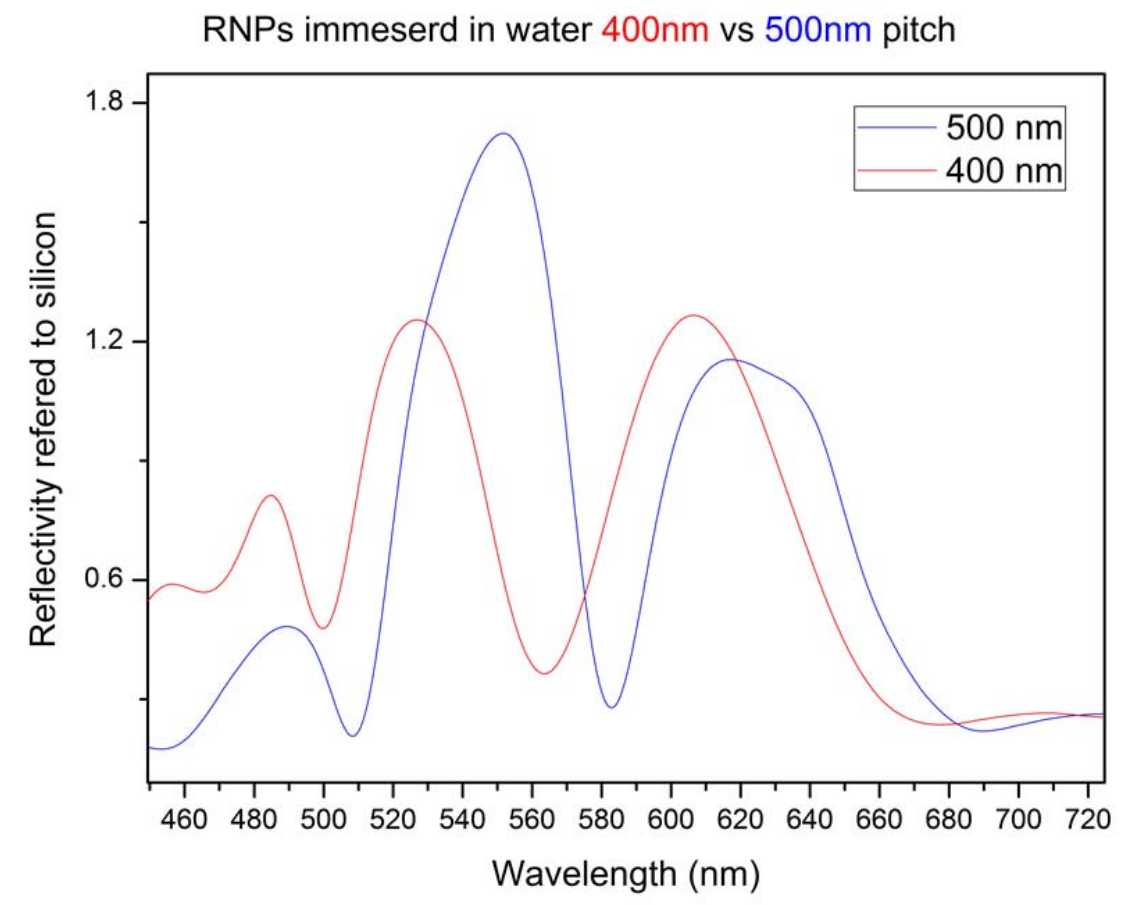

Figure 72: Optical response of $R$-NPs immersed in water with two different lattice parameter, 400 and $500 \mathrm{~nm}$.

For the experiment it was used the optical set-up based on a bundle of fiber and a spectrometer covering the visible range, mounted in the laboratory of GOPB. (Chapter 6). Each of the samples was composed by eight independent cells. Bulk 
sensing experiment was developed by measuring seven times, four different RI fluids: water (1.33), ethanol (1.36), hexane (1.375) and toluene (1.49) in the eight different cells.
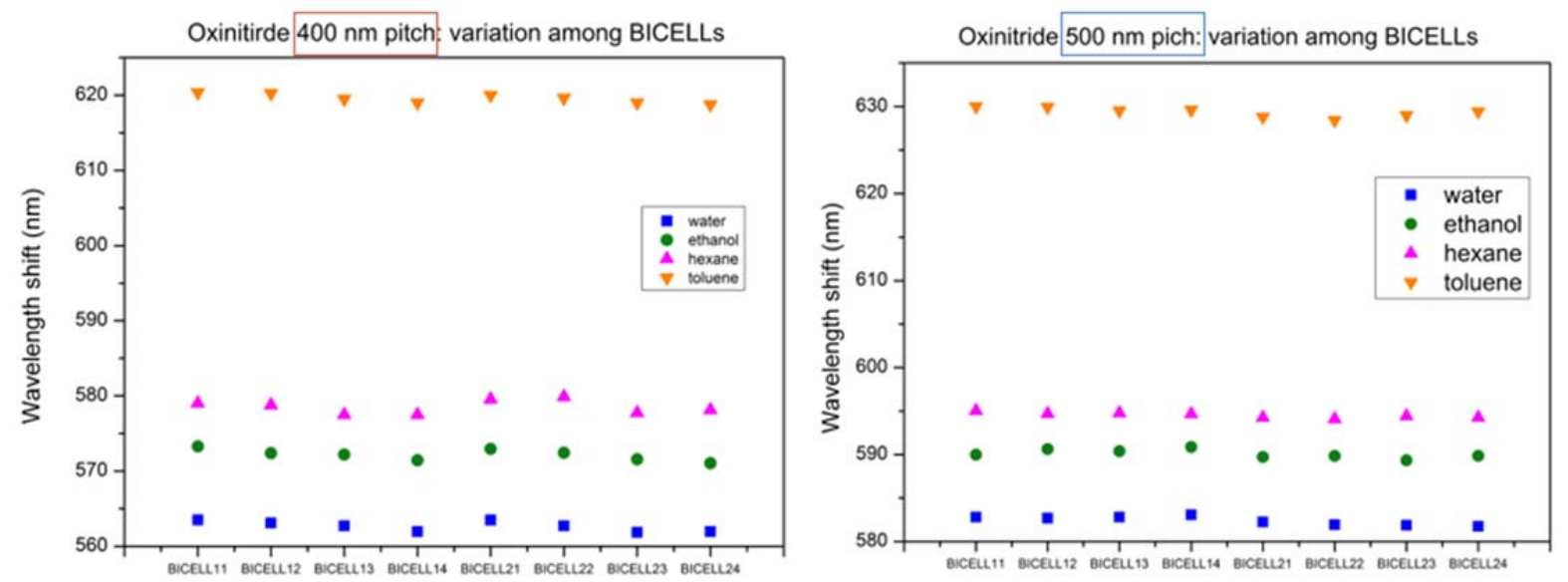
Figure 73: Bulk sensing experiment with different solvents in R-NPs with 400 and $500 \mathrm{~nm}$ of
pitch, measuring all the R-NPs arrays from each chip.

In both samples, homogeneity among the cells seems to be relatively good with certain dispersion. The total wavelength shift from water to toluene is of $60 \mathrm{~nm}$ in $400 \mathrm{~nm}$ pitch samples and $50 \mathrm{~nm}$ in $500 \mathrm{~nm}$ pitch samples. Despite this, the homogeneity seems to be higher in the new lattice parameter samples. Besides the reproducibility among different fabrication batches resulted to be more stable in these last sample.

In the next table it is resumed the mean position the resonance mode and the deviation, for the different cells, for the different fluids measured for both lattice parameter tested: 
Table 3: Bulk sensing comparison data between R-NPs with 400 and $500 \mathrm{~nm}$ of lattice parameter.

\begin{tabular}{|c|c|c|c|c|c|c|c|c|}
\hline $\begin{array}{l}\text { Fluid } \\
\text { RI }\end{array}$ & \multicolumn{2}{|c|}{$\begin{array}{l}\text { WATER } \\
1.33\end{array}$} & \multicolumn{2}{|c|}{$\begin{array}{l}\text { ETHANOL } \\
1.36\end{array}$} & \multicolumn{2}{|c|}{$\begin{array}{l}\text { HEXANE } \\
1.375\end{array}$} & \multicolumn{2}{|c|}{$\begin{array}{l}\text { TOLUENE } \\
1.49\end{array}$} \\
\hline $\begin{array}{c}\text { LATTICE } \\
\text { PARAMETER } \\
(\mathrm{nm})\end{array}$ & 400 & 500 & 400 & 500 & 400 & 500 & 400 & 500 \\
\hline $\begin{array}{c}\text { MEAN } \\
\text { POSITION OF } \\
\text { THE } \\
\text { RESONANCE } \\
\text { (nm) }\end{array}$ & 562.7 & 582.3 & 572.3 & 590.0 & 578.4 & 594.5 & 619.5 & 629.3 \\
\hline $\begin{array}{c}\text { STANDARD } \\
\text { DEVIATION OF } \\
\text { THE POSITION } \\
\text { (nm) }\end{array}$ & 0.61 & 0.50 & 0.78 & 0.51 & 0.92 & 0.31 & 0.61 & 0.55 \\
\hline
\end{tabular}

Maximum deviation observed is $0.92 \mathrm{~nm}$ for the hexane measurement in $400 \mathrm{~nm}$ pitch samples. And the lowest was $0.31 \mathrm{~nm}$ for the same solvent but in the $500 \mathrm{~nm}$ pitch samples. This could be due to the fabrication process, which is more robust in samples with a lattice parameter of $500 \mathrm{~nm}$.

It means that $400 \mathrm{~nm}$ pitch samples can be perfectly used as biochemical sensors, but the parameters used in their manufacturing process make them less homogeneous and reproducible than $500 \mathrm{~nm}$ pitch samples.

For the comparison of the performance of both type of transducers, it was calculated the bulk sensitivity of both samples. 


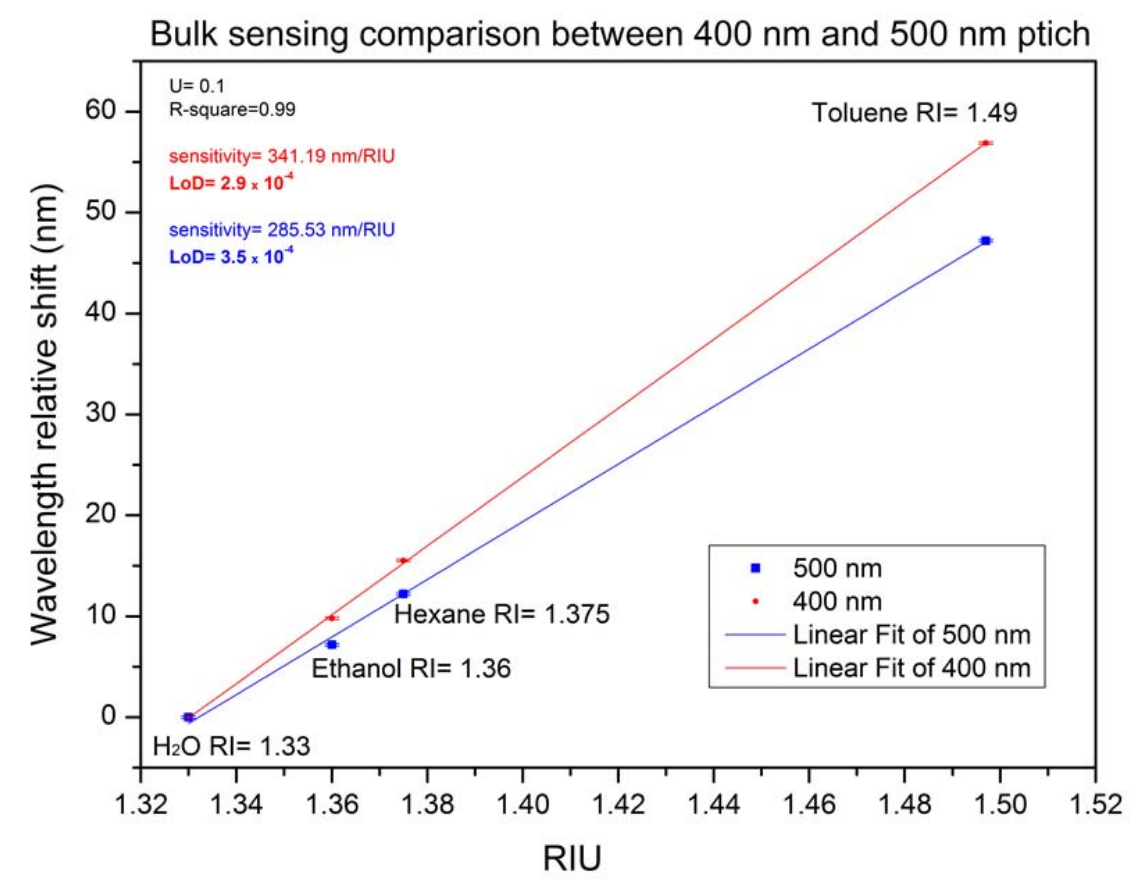

Figure 74: Comparison of the bulk sensitivity of the two different R-NPs pitches, 400 and $500 \mathrm{~nm}$.

Sensitivity in samples with $500 \mathrm{~nm}$ of lattice parameter (285.53 nm/ RIU) is lower than sensitivity in samples with a pitch of $400 \mathrm{~nm}(341 \mathrm{~nm} / \mathrm{RIU})$. The variance $\left(s^{2}\right)$ observed for the seven repeated measurements was in the order of $10^{-4}$, therefore with this optical set-up the uncertainty was mainly influenced by the resolution of the system $0.12 \mathrm{~nm}$ (equation 9).

Even though the sensitivity was slightly lower for the R-NPs with $500 \mathrm{~nm}$ pitch, the limit of detection remained in the same order of magnitude, very similar. It decreased from $3.5 \times 10^{-4}$ to $2.9 \times 10^{-4}$ respectively.

Thus, because the decrease of sensitivity of the R-NPs with $500 \mathrm{~nm}$ pitch was not very signifficative, and due to the increased fabrication homogeneity found in R-NPs of $500 \mathrm{~nm}$ pitch, it was decided to continue working with samples with R-NPs with $500 \mathrm{~nm}$ of lattice parameter.

It must be also noticed that the sensitivity of samples of $400 \mathrm{~nm}$ pitch found in this experiment, $341 \mathrm{~nm} / \mathrm{RIU}$, is smaller than the $591.94 \mathrm{~nm} / \mathrm{RIU}$ found for the first experiments accomplished for the redesigned R-NPs. (See subchapter 7.5). 
The reason is the methodology used for the fluid RI estimation. In the first case, there were used fractions of water-ethanol and the indexes of the fluids were estimated according to the equation 17. Then, for the comparison of the samples of different pitches, the fluids measured were not fractions of water/ethanol, but there were used different solvents, water, ethanol, hexane and toluene with a defined RI in the scientific literature.

Hereafter is presented a table summarizing the optical performance parameters of the two different R-NPs lattice parameters studied in this bulk sensing experiment:

Table 4: Performance results of the R-NPs with $400 \mathrm{~nm}$ and $500 \mathrm{~nm}$ of lattice parameter.

\begin{tabular}{|c|c|c|}
\hline Samples & $400 \mathrm{~nm}$ pitch & $500 \mathrm{~nm}$ pitch \\
\hline $\begin{array}{c}\text { Sensitivity } \\
\text { (nm/RIU) }\end{array}$ & 341.19 & 285.53 \\
\hline LoD & $2.9 \times 10^{-4}$ & $3.5 \times 10^{-4}$ \\
\hline
\end{tabular}

\subsection{Continuous bulk sensing}

All the liquid infiltrations in the R-NPs measured in the former subchapter were performed in static conditions. The fluids were introduced and removed from the holder manually, thus the interphase between two different fluids was unable to be monitored. Nevertheless, it is really important to know the real-time sensing response of the R-NPs during fluids changes, especially in order to study the kinetics of the reactions of the bioreceptor and the analyte in biological detection, and detect the biological reactions as they happen. For this reason there were accomplished two different continuous flow experiments with R-NPs introduced in a flow cell. One, by measuring the optical transmittance from the optical absorbance measured with a spectrophotometer, and other by measuring reflectivity profiles with the already described optical set-up from the GOPB. 
7.7.1 Real-Time response as a function of the absorbance

The focus of this experiment was to demonstrate if the R-NPs were able to provide real-time response. [Fernandez, 2016 $]$

For this experiment it was a used a peristaltic pump and a common spectrophotometer by adapting the sensing cuvette to the R-NPs chips dimension, to be used as the fluidic holder or flow cell. In this case the R-NPs were not distributed in arrays, but the whole chip was nanostructured with the R-NPs. The fluidic holder used for the fluid circulation had already been used for LSPR flow cells. [Fernandez, 2016 ${ }^{b}$ ]

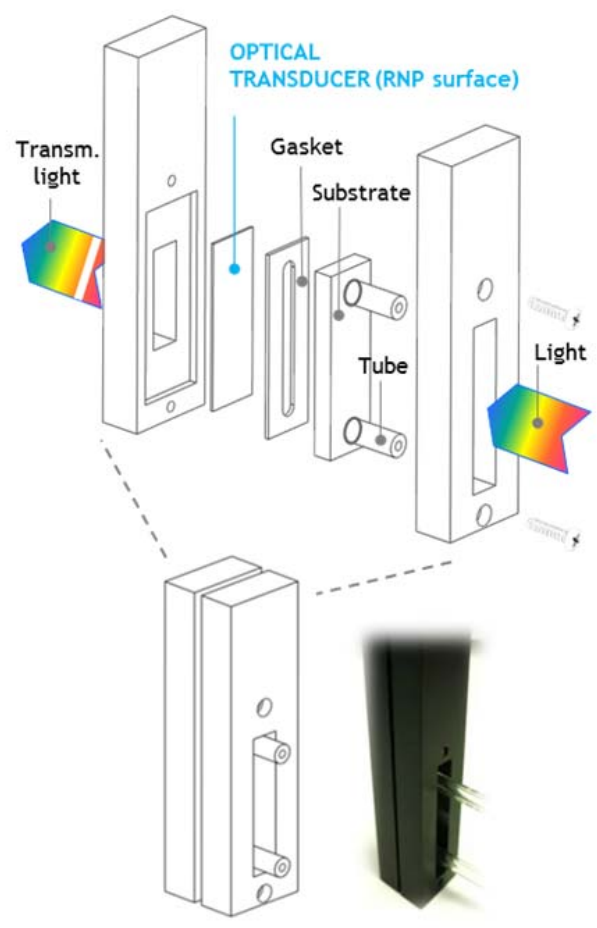

FLUIDIC HOLDER FOR MEASUREMENTS
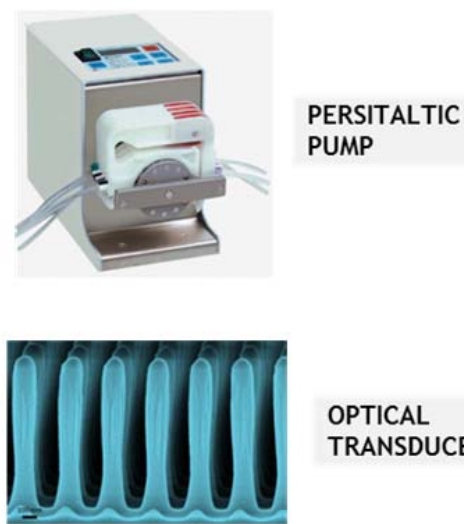

OPTICAL

TRANSDUCER

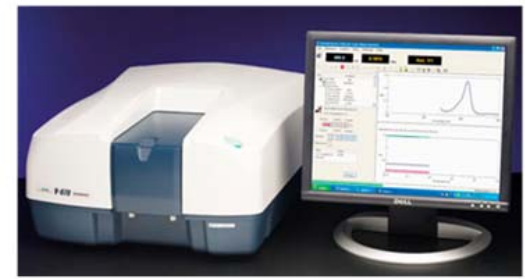

SPECTROPHOTOMETER

Figure 75: On the left. Adaptation of the espectrophotometer flow cell for R-NPs measurement. On the right. All the elemts involved: peristaltic pump, R-NPs chip, spectrophotometer.

Optical signals (OS) in transmission mode, were obtained by interrogating a large area of the sensing chip. Thus, resonance modes were not studied, but only the OS variation as afunction the RI of the flowing fluids. Then, for this approach there 
were used the $\mathrm{Si}_{3} \mathrm{~N}_{4} / \mathrm{SiO}_{2}$ R-NPs based on 18 Bragg reflector pairs and the central cavity with a lattice parameter of $600 \mathrm{~nm}$. The OS value was calculated as logarithmic ratio of the intensity of transmitted $(I)$ and incident $\left(I_{0}\right)$ radiation through the R-NP transducer $\left(-\log \left(I_{0} / I\right)\right)$.

For the real-time response, each of the solutions was consecutively introduced in the flow cell during 5 minutes at a rate of $50 \mu \mathrm{L} / \mathrm{min}: 0,4,9.8,15,21,15,8.9,4$ and $0 \%$ of $\mathrm{NaCl}$ in $\mathrm{Di}-\mathrm{H}_{2} \mathrm{O}$. Corresponding RI values are: 1.3300 (0\%), 1.3400 (4\%), 1.3502 (9.8\%), $1.3594(15 \%)$ and 1.3700 (21\%).

For the proper assessment of the best wavelength to accomplish the real-time test, there were constructed linear regressions for the different wavelength representing the increment of the $\mathrm{OS}(\triangle \mathrm{OS})$ as a function of the RI variation (RIUs). (Figure B). Specifically, the most suitable wavelength seemed to be $383 \mathrm{~nm}$, due to its greater sensitivity (33.45 OS/RIU) regarding to the others wavelength studied. (Figure 76. A). It is worth to highlight that the sensitivity was 5 times bigger that the sensitivity found for LSPR (6.65 OS/RIU) measured with the same fluid system.

Real-time response was acquired by monitoring the optical signal at a constant wavelength $(383 \mathrm{~nm}, 503 \mathrm{~nm}$ and LSPR $840 \mathrm{~nm})$, while pumping the different solutions. The measures of the OS were acquired every 0.5 seconds. 

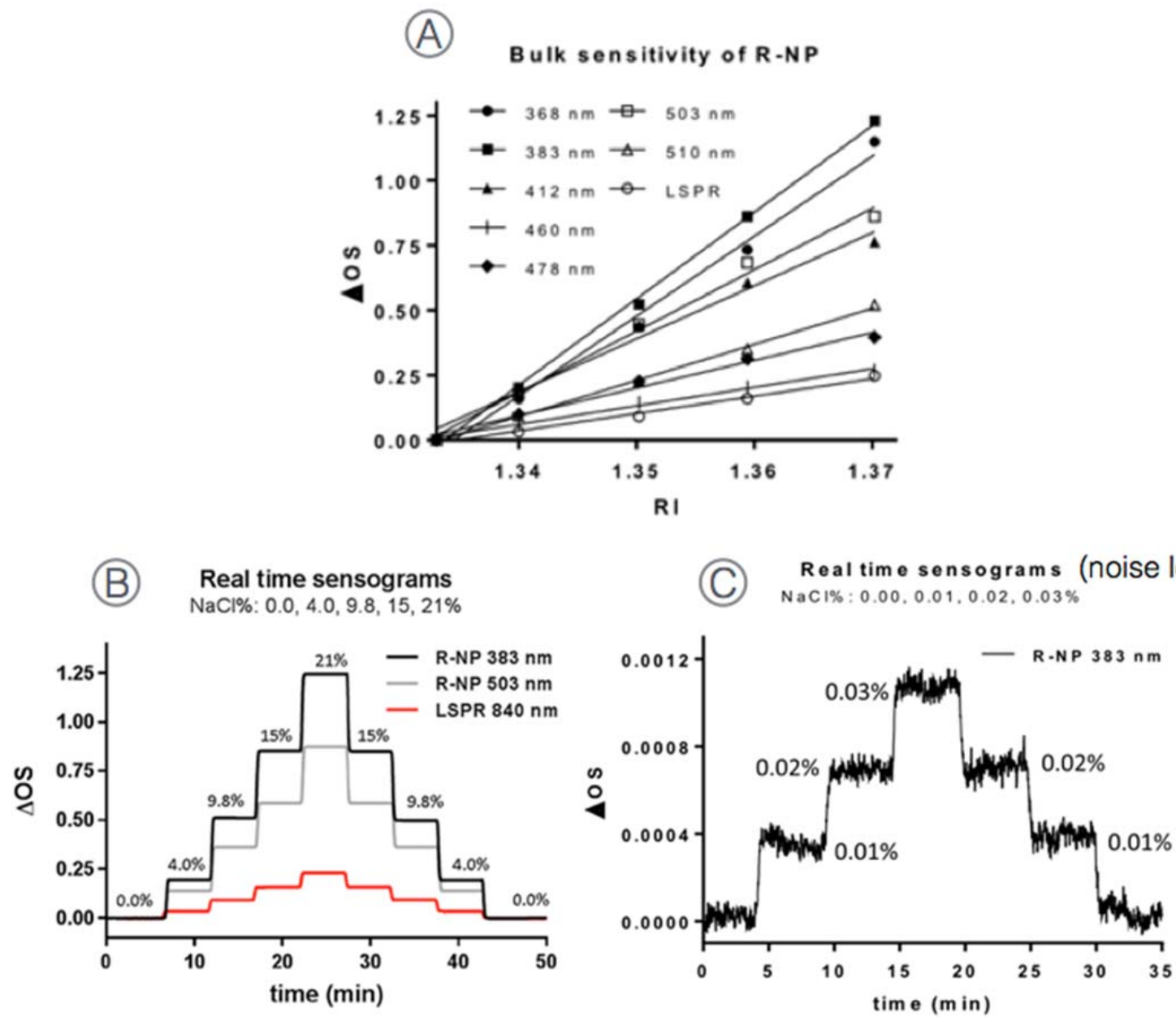

Figure 76: A. Sensitivity (OSvs.RI) of the R-NPs system at different wavelengths. Sensitivity of a LSPR transducer is also included ( = 840 nm). B. Response to RI 1.330-1.370 $(0.0-21 \% \mathrm{NaCl})$ for R-NPs at $383 \mathrm{~nm}$ (black line), R-NPs at $503 \mathrm{~nm}$ (grey line) and LSPR at $840 \mathrm{~nm}$ (red line). C. Real time sensogram (OS vs. time) for R-NPs at $383 \mathrm{~nm}$. Response to 0.00, 0.01, 0.02 and $0.03 \%$ of $\mathrm{NaCl}$. Each $0.01 \%$ of $\mathrm{NaCl}$ is equivalent to $1.8 \cdot 10^{-5}$ increment in $\mathrm{RI}$.

For the estimation of the limit of detection $(L o D=U / m)$ of the three different realtime sensograms, the $U$ was considered as the resolution of the optical signal, by multiplying the noise level $(N)$ per a factor of $3(U=R=3 N)$. For this purpose, the fluctuation of the signal, or what is the same, the noise of the signal, was determined from a sensogram when a solution is flowing stably. The mean noise levels observed were $0.00020,0.00023$ and 0.00015 OS for R-NPs $383 \mathrm{~nm}$, R-NPs $503 \mathrm{~nm}$ and LSPR respectively. These values suggest a very stable real time response. Taking into account noise levels and sensitivities $(m)$ the achieved LoD was $1.8 \times 10^{-5}$ for R-NPs $383 \mathrm{~nm}, 3 \times 10^{-5}$ for R-NPs 503 and $6.7 \times 10^{-5}$ for LSPR $(840 \mathrm{~nm})$. These values are the minimum variation of RI that generates a detectable signal. To confirm this detectability limit, R-NPs were loaded with solutions of 
Di- $\mathrm{H}_{2} \mathrm{O}$ and $0,0.01,0.02$ and $0.03 \%$ of $\mathrm{NaCl}$ and monitored at $383 \mathrm{~nm}$ (Figure 76.C). Very small changes in RI could be observed.

In conclusion, these experiments demonstrated on one side the ability of R-NPs arrays to provide a sensing response in real-time, fulfilling thus, one of the most relevant requirements of the Enviguard Project. On the other side it was showed that the R-NPs bulk sensitivity (33 OS/RIU) and the limit of detection $\left(1.8 \times 10^{-5}\right.$ RIU) were improved 5 and 3.7 respectively, regarding previous LSPR sensitivity and LoD values found.

\subsubsection{Real-time response measured in reflectance}

Once the monitoring of the R-NPs in real-time was demonstrated, it was necessary to evaluate the real-time response of the R-NPs arrays measured in reflectometry.

For this continuous flow experiments it was used the fluidic system fabricated by Lionix. The sample chip with eight R-NPs arrays of $500 \mathrm{~nm}$ pitch R-NPs was introduced in the flow cell, of the fluidic system and R-NPs arrays were independently interrogated with the optical set-up mounted in the laboratory of the GOPB, specifically for that purpose.

Each of the reservoirs of the fluidic system was filled with increasing Di- $\mathrm{H}_{2} \mathrm{O}$-ethanol fraction (\%): 0, 5, 10, 20, 40 and 100. Each increasing solution was loaded over the flow cell, during 15 minutes continuously one after another. When reached $100 \%$ of ethanol, the process continued in reverse by loading decreasing fractions of ethanol-water until $\mathrm{Di}-\mathrm{H}_{2} \mathrm{O}$ baseline measurement. Flow rate was also $50 \mu \mathrm{L} / \mathrm{min}$, but the tubing were longer than the tubing used in the former continuous flow experiment, therefore the consumed time in total was 180 minutes. 


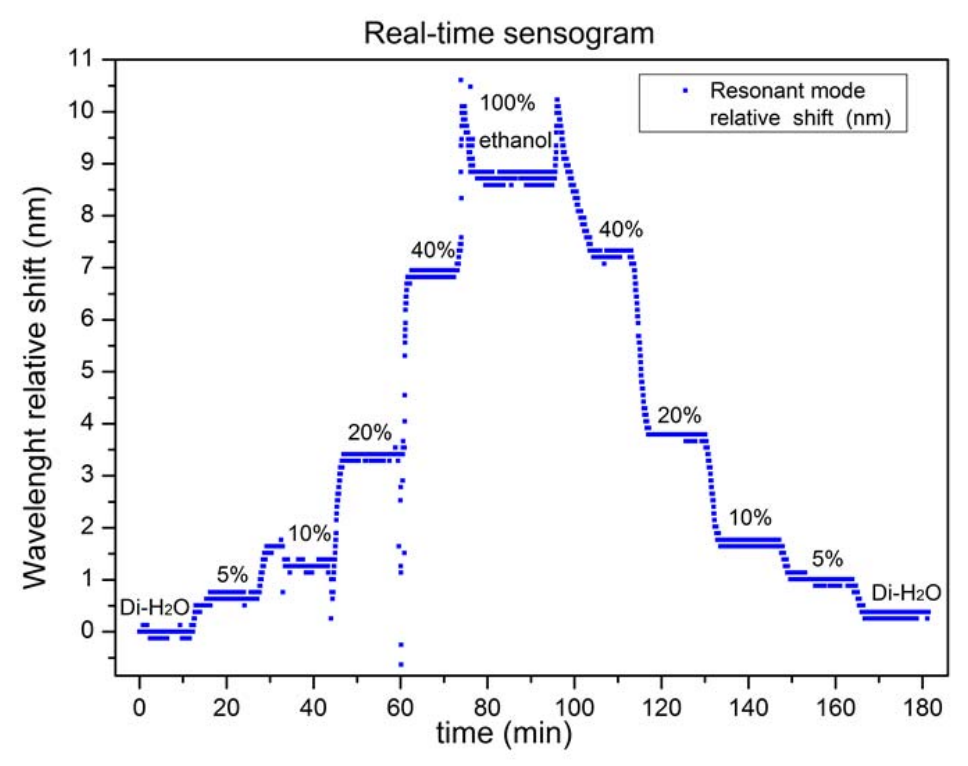

Figure 77: Real time response of the R-NPs for diffrent RI fluids pumping as afunction of the wavelenght.

In figure 77 it is represented a chart of the real time response of one R-NPs array. As the different fluids get in contact with the R-NPs, the RI of the resonant cavity changes and thus the position of the resonant mode in reflectivity the measurements. The response was monitored by measuring the position of the resonant mode every 1.7 seconds. In the chart, the $\mathrm{Y}$ exe is the relative shift of the resonant mode as a function of the time in minutes ( $\mathrm{X}$ exe). For that purpose it was used the specific software developed by the CEI from UPM adapted to the Mightex spectrometer to permit the continuous measuring of the optical spectra and the resonant mode detection.

It is remarkable from figure 77, the acute slopes between two different loading fluids. These slopes suggest a phase of mixing fluids in the tubing of the system, which reaches the stable flat area when the fluid is no longer mixed. The frequency of the points of those slopes is limited on one side, by the acquired data by time unit ( 2 seconds in this case) and on the other side, by the resolution of the spectrometer, $0.1 \mathrm{~nm}$. 
With this experiment it was demonstrated the possibility of measuring R-NPs in reflectometry and monitoring the resonance position in real time. In this case it was interrogated only one BICELL because, the optical set-up has one single fiber bundle.

Even though, after fluidic system tests success, it was developed a chemical detection unit prototype (CDU) for Enviguard Project, with a similar fluidic system and a flow cell, integrated into an optical head, able to record the real-time response of the different R-NPs arrays simultaneously, by monitoring resonance modes along the time. (Figures 78 and 79).

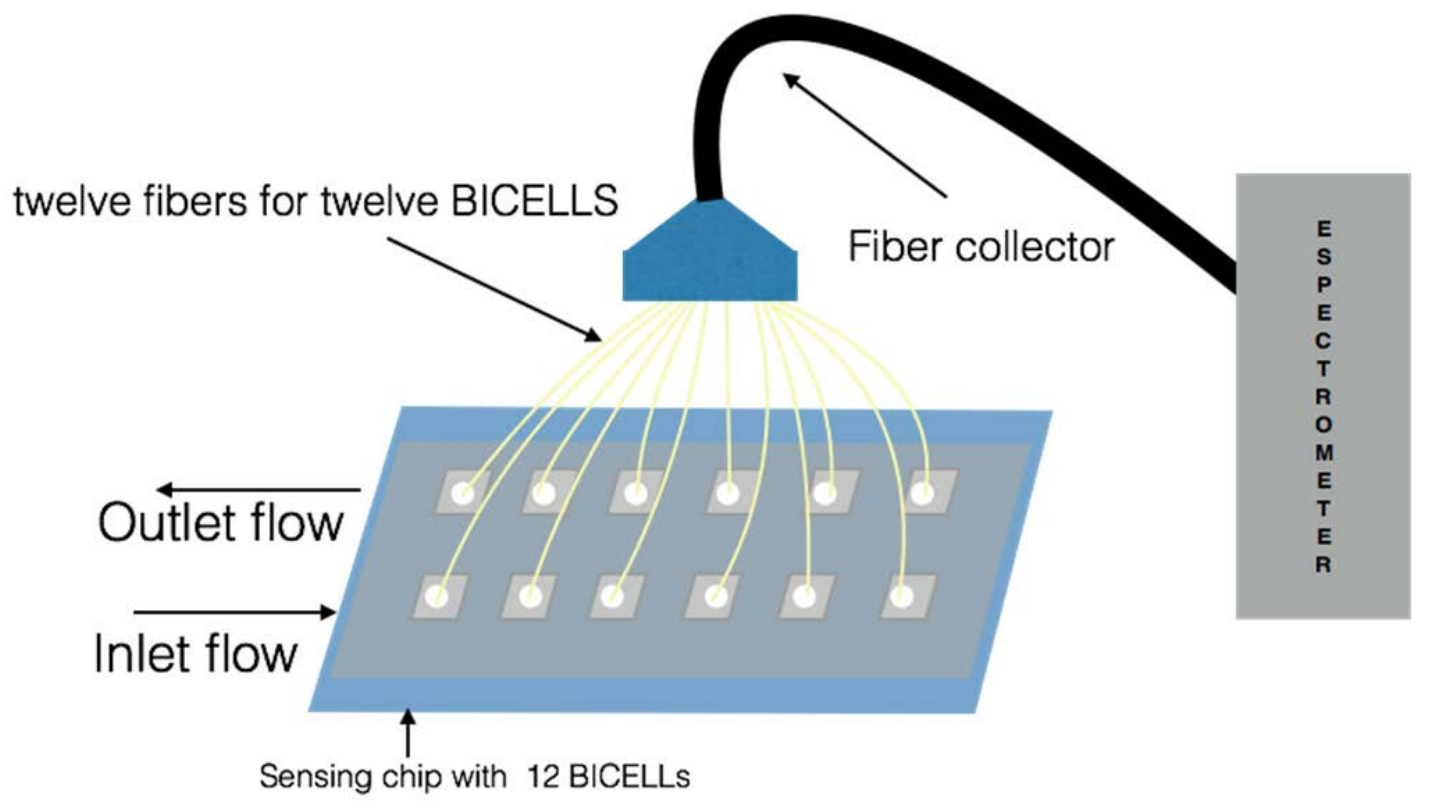

Figure 78: Scheme of the simultaneous interrogation of the twelve R-NPs arrays. 

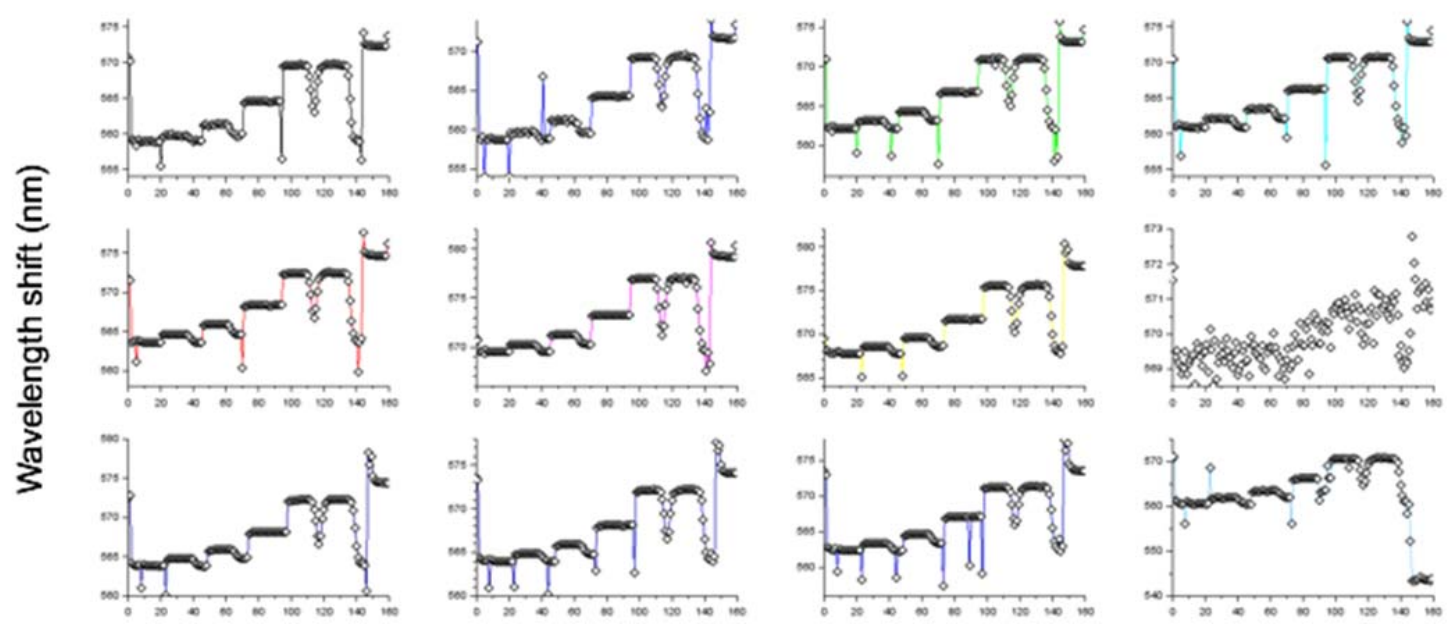

Figure 79: Simultaneous response of the twelve different R-NPs arrays in real time measuremnts of several RI fluids.

The final chip used for Enviguard consisted in twelve R-NPs arrays to test three different analyte and the reference fluid to be measured by triplicate. Therefore the optical head was composed by twelve optical fiber connected to a spectrometer of a very high resolution ( $5 \mathrm{pm}$ ), so small concentrations of low weight analyte could be detected.

The optimum parameters of a fluidic system and the optical read-out system must be in concordance with the application being measured. It must be a compromise between the amount of data acquired and the amount of time consumed.

For example for biochemical detection experiments the time frequency of acquiring data must be higher, because the change of fluid is a fast process. Nevertheless, biological reactions normally take longer times and the time frequency can be decreased so that the amount of data recorded is manageable.

\subsection{Summary}

The results obtained in this work seem to be really promising expecting further improvements in future work in order to enhance the optical response of the 
R-NPs arrays transducers. For example, the Q-factor of the resonance dips could be improved by increasing the number of Bragg reflectors (and thus the height of the pillars), or by using other materials with a higher refractive index contrast. Other improvements could result from reducing the diameter of the R-NPs and the pitch among the R-NPs. These aspects will have significant implications for enhancing the sensitivity, although they involve complexities under the micronano fabrication perspective. Finally, due to the capability of detecting small variations of RI fluids, it is expected that these types of R-NPs arrays transducers can be applied in the detection of biological agents and biomolecules such as environmental toxins, chemical pollutants, and biomarkers, among others.

In conclusion, in this chapter, it is shown the experimental response of R-NPs arrays as RI transducers, with a significant performance improvement. As this was equal to or improved upon the other reported transducers in the literature, this work seems a promising approach for future label-free biosensing. 


\section{R-NPS ARRAYS FOR LABEL-FREE BIOSENSORS}

In this chapter it is studied the capacity of the R-NPs to immobilize biomolecules and to detect subsequent molecular interactions. To do this, first it is explained what a biofunctionalization protocol consist of, and which strategies were tested on flat silicon oxide interferometers. Secondly, there are shown the results of an immunoassay developed over R-NPs. The optical interrogation of the R-NPs during the immunoassay steps was performed with two different methodologies: firstly with the R-NPs in dry conditions, and secondly with the R-NPs immersed in water. Also it is studied in this chapter, the effect of carrying out the immobilization of the bioreceptor in continuous flow with the fluidic system. After works, there are compared the performances of the different interrogation methodologies (R-NP dry vs R-NP in contact with water) and the different procedures of immobilization of the IgG over R-NPs (static vs continuous flow immobilization). Finally there are shown the improvements achieved in the international stay at Wageningen University, regarding surface modification strategies for biofunctionalization protocols developed over silicon oxide surfaces.

\subsection{Biofunctionalization protocol}

Optical transducers provide the evidence of the reaction taking place between the bioreceptor and the target analyte, for that reason the immobilization of the bioreceptor on the transducer is a key factor in biosensors development. [Bañuls, 2013]. The whole process involving the bioreceptor attachment is called the biofunctionalization protocol and very often it includes surface modification strategies, in order to enhance and facilitate the bioreceptor immobilization over the desired surface material. For a better performance of the biosensor, the modified surface monolayer must be able to attach covalently homogeneously and properly oriented bioreceptors. This monolayer should also prevent matrix effect of the samples and the unspecific attachment of the biomolecules [Cecchet, 2005]. Those complex requirements need to be attained to really obtain a good quality 
biosensor throughput. [Myzska, 1999]. However biochemistry is a delicate science in which small changes in the experimental conditions can have notable effect on the obtained results, and thus decent of parametric experiments must be performed in order to obtain a biofunctionalization protocol stable and reproducible.

For the biofunctionalization of the R-NPs and the further recognition of the analyte, there were studied two different surface modification approaches based on the silane chemistry over $\mathrm{SiO}_{2}$ flat interferometers. There exist many types of silanes according to their binding sites, chain size, functional groups and some other characteristics.

The goal of the silanization strategies is to create a stable monolayer covalently attached to the surface, with a functional group able to bind bioreceptor through covalent bonds. Nevertheless, the binding position of the bioreceptor cannot be random, and the choose of the adequate silane must be focused on the immobilization of the bioreceptor through the appropriate chemical group, in order to enhance the later analyte recognition.

(A) Oriented immobilization

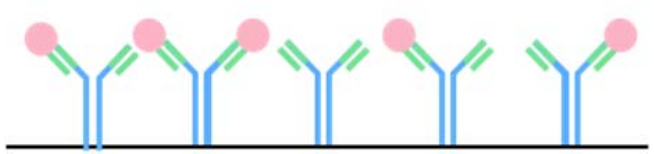

(B) Random immobilization

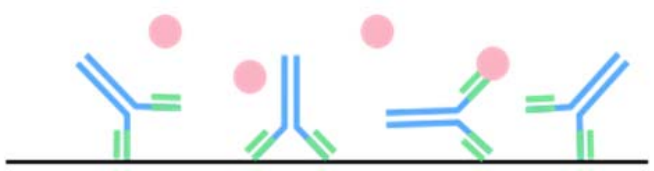

Figure 80: A. Oriented immobilization antibodies. B. Random immobilization of the antibodies.

In figure 80.A it is showed the immobilization of the antibodies through constant region. Fabs remain free for the antigen binding. Figure 80. B represents different possibilities of random immobilization of the antibody in which antigen binding sites are not totally available. [Trilling, 2013]. 
For the determination of the most adequate biofunctionalization protocol, two different silanes (purchased in Sigma Aldrich) were studied: 3-Glycidyloxypropyl trimethoxysilane (GPTMS) and Aminopropyl trimethoxysilane (APTMS).

For the experiments developed in this chapter, related to biological sensing, it was used the same bioapplication. It consisted on IgG/anti-IgG complex purchased from Sigma Aldrich. The IgG from rat serum (I4131), is widely used as antigen in common immunoassays. Anti-rat IgG (R3756), is a polyclonal antibody, obtained from IgG purified from pooled normal rat serum as the immunogen. Thus, in this immunoreaction there participate two antibodies, one acting as the antigen (IgG) and the other as the antibody (anti-IgG). Since anti-IgG is polyclonal antibody and was developed by using IgG as the immunogenic compound, it can use its Fab groups to create bonds with different binding areas of the IgG.

\subsubsection{Stages of the biofunctionalization protocol}

There exist a wide range of biofunctionalization protocols according to the different surface modification technics and specific requirements and conditions of every kind of label-free biosensors. The following lines summarize the typical steps carried out on biofunctionalization protocols based on silane chemistry, which were followed in this work.

\section{- Cleaning}

Surface modification with silane requires a specific pre-treatment of the siliconbased surface area. It must be properly cleaned, (Figure 84. A) in order to get rid of any inorganic or organic, micro- or nano-particles, preventing or altering the surface modification. For the cleaning, surfaces can be sonicated with different chemical reagents such as ethanol, acetone, toluene, dicloromethane (DCM), among others [Henke, 2002]. 


\section{- Activation}

In addition the silicon oxide surfaces need to be chemically activated generating $-\mathrm{OH}$ radical groups on the surface to facilitate the silane binding. The activation (figure 81 and 84. B) can be performed through Piranha solution in different proportions $\left(\mathrm{H}_{2} \mathrm{SO}_{4}: \mathrm{H}_{2} \mathrm{O}_{2}\right)$ or by plasma oxygen. This step helps to eliminate water molecules, which are immiscible with silane compounds, and to remove definitely the organic material remaining in the surface thanks to the extremely reactive oxygen ions generated in both process.
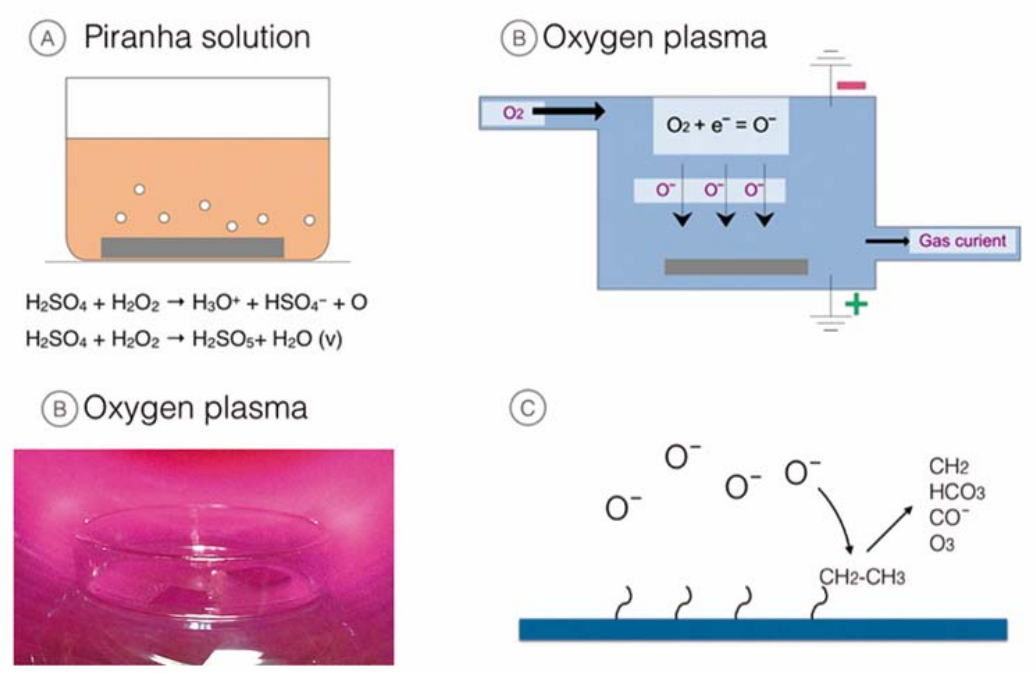

Figure 81: Methods for surface activation. A. Piranha activation. B. Oxygen plasma activation. C. Oxigen radicals for surface activation.

\section{- Silanization}

Once the surface is cleaned and activated, it is brought in contact with the silane dissolved in anhydrous organic solvent (ethanol, methanol, toluene) or in pure conditions. It will depend on the silane type and final purpose. In order to make sure the silanization of the surface is complete, and successful, this process is normally accomplished overnight and many times in Argon or Nitrogen atmosphere. Even though, some authors claim that by adjusting the percentage of silane in the solvent it can be reduced the silanization time. [Gunda, 2014]. In this 
work two different silanes were tested: GPTMS and APTMS. Both have three metoxy groups $\left(-\mathrm{OCH}_{3}\right)$ that, through the oxygen, react with the $-\mathrm{OH}$ groups formed in the activated surface. GPTMS has an epoxy group as functional group in contrast to APTMS that has an amine group.

Then, according to the functional group of the silane, remaining in the surface, the bioreceptor can be immobilized directly. But sometimes it is necessary to use a coupling chemistry for the activation of the functional groups remaining in the surface after silanization. [Ling, 2006]. For the APTMS silanization in this work, it was used glutaraldehyde (GA) molecule formed by two aldehydes as functional groups and three carbons in between acting as spacer. One aldehyde binds to the amine group of the APTMS and the other aldehyde binds to the amine group from the bioreceptor, which was in this case an IgG.

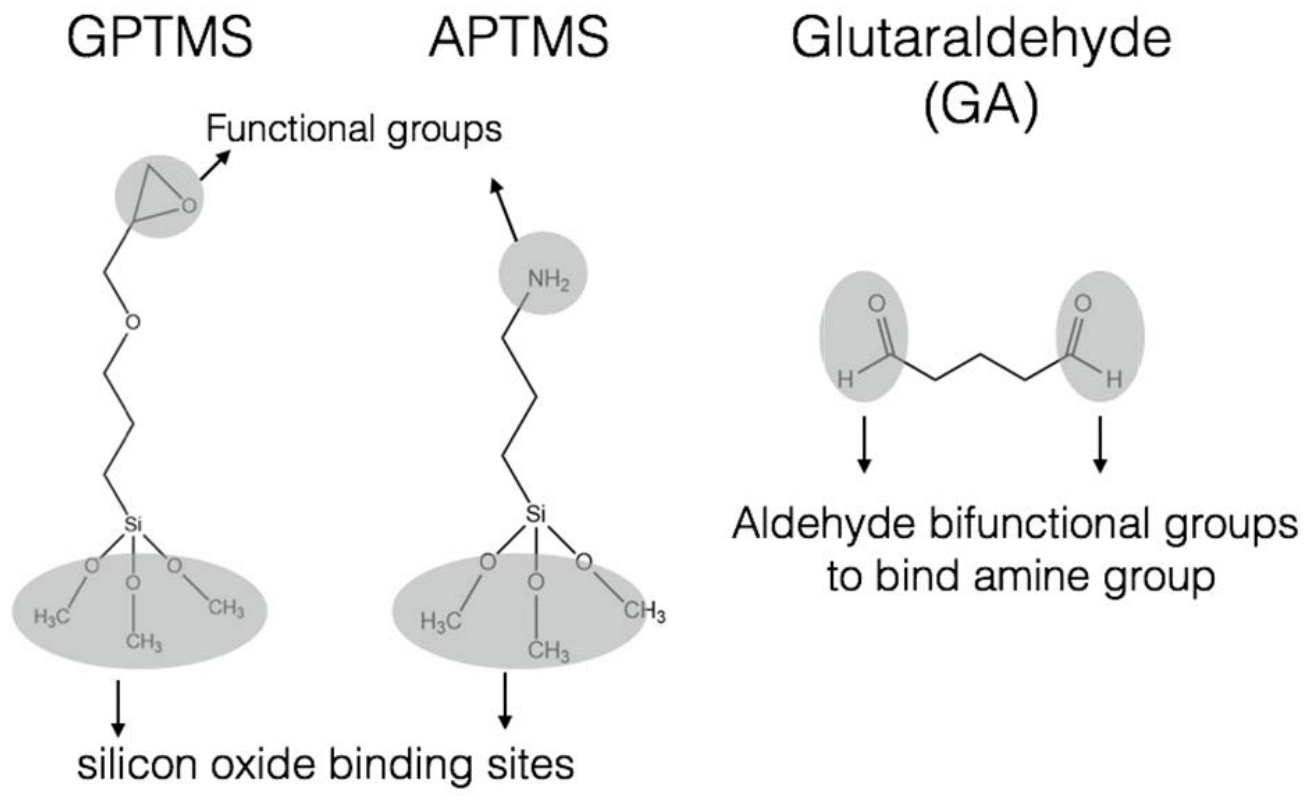

Figure 82: Silanes used structure and glutaraldehyde linker structure.

The specific parameters used for silanization protocols in this thesis are detailed subchapter 8.1.2, in the experimental results. 


\section{- Immobilization}

The incubation time for the bioreceptor immobilization over the silanized surface, depends on the nature of the biomolecules and on the process of immobilization which can be in static of in continuous flow. For the static immobilization, in this work a drop of $50 \mu \mathrm{L}$ of $\operatorname{IgG} 50 \mu \mathrm{g} / \mathrm{mL}$ in PBS was incubated overnight in humid conditions at $37^{\circ} \mathrm{C}$. After this, the sample was gently washed with a syringe coupled to a $0.4 \mu \mathrm{m}$ pore size filter, with $20 \mathrm{~mL}$ of $\mathrm{Di}-\mathrm{H}_{2} \mathrm{O}$ and $20 \mathrm{~mL}$ of $1 \%$ Tween PBS (PBS-T), to remove the non attached material. Then, for the optical interrogation in dry conditions, which means that the interferometer must be totally dry, it was properly dried with clean air. For the immobilization of the bioreceptor in continuous flow, incubation times can be considerably reduced, and surfaces do not need to be dried since in continuous flow, samples are interrogated in fluidic conditions.

After this last step, if everything went well, it would be obtained a biofunctionalized surface or transducer, giving practical meaning to the biosensor.

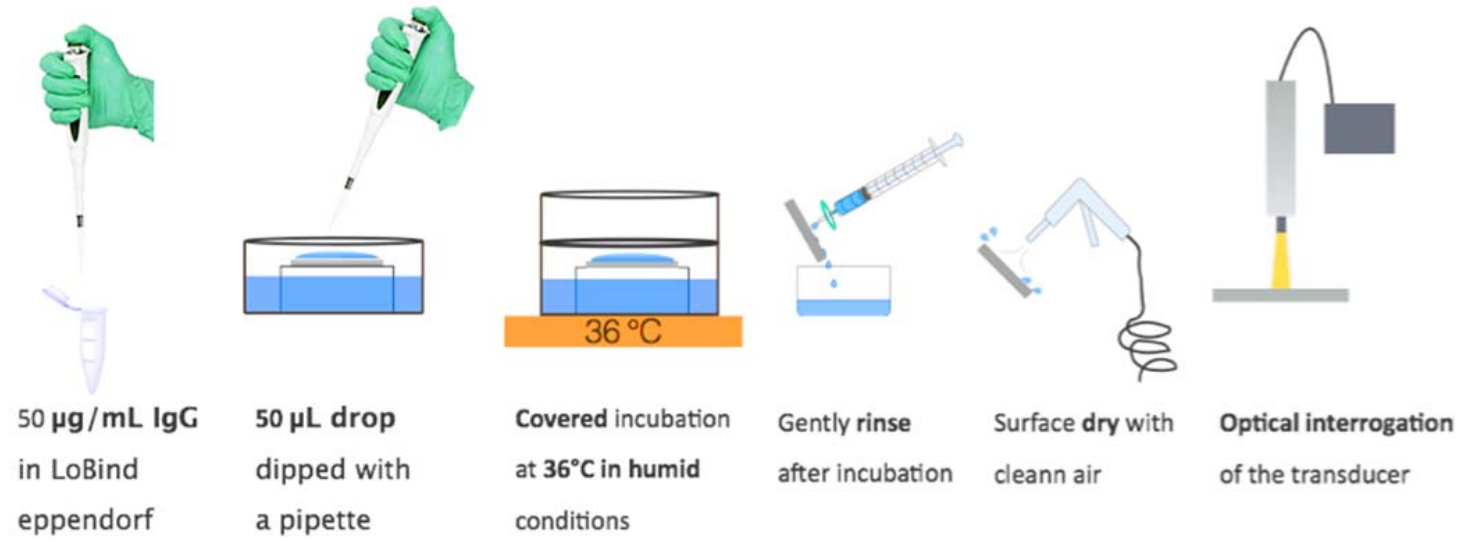

Figure 83: Immobilization protol steps for the antibody detection.

All the steps contained in the process, from the cleaning of the surfaces to the immobilization of the bioreceptor, compose the biofunctionalization protocol (Figure 84). The parameters of the process (cleaning reagents, silanes used, coupling agents, incubation times, etc.) must be optimized according to each type 
of interferometer materials and structures and also, to the nature of the bioreceptor.

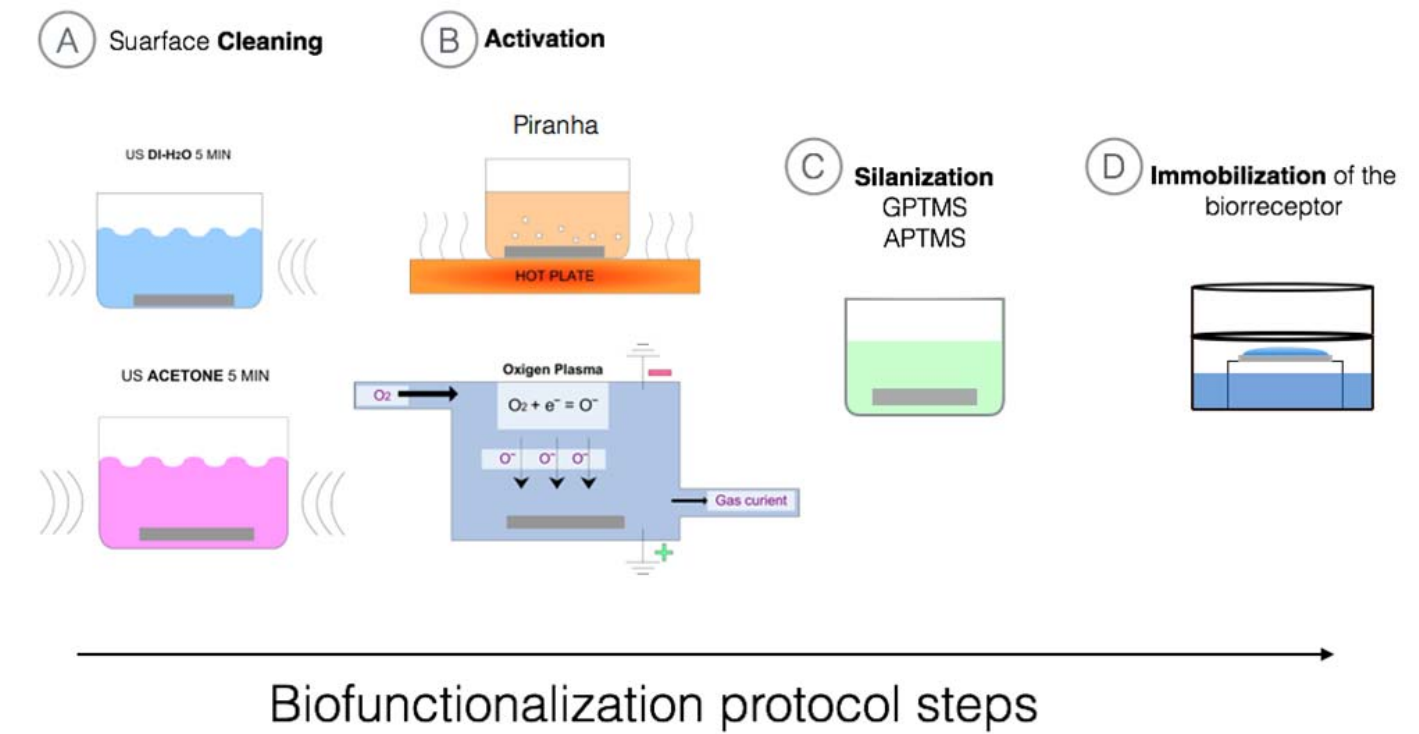

Figure 84: Bifunctionalization steps: A. Cleaning of the surface with different solvents. B. Activation of the surface. C. Surface modification through silane chemistry. D. Incubation of the bioreceptor in static conditions.

\subsubsection{Biofunctionalization strategies carried out within this work}

Two different biofunctionalization protocols based on two different silanes (GPTMS and APTMS) were tested over $600 \mathrm{~nm}$ of $\mathrm{SiO}_{2}$ over silicon substrate, flat surfaces, so R-NPs samples were not employed for these first approaches.

The reason for using $\mathrm{SiO}_{2}$ surfaces is that it is the material that makes up the majority of the surface of the R-NPs. Specially the resonant cavity, which is the most sensitive area of the R-NPs. Thus, it is necessary to understand the $\mathrm{SiO}_{2}$ behavior after chemical treatments. The $\mathrm{Si}_{3} \mathrm{~N}_{4}$ is also present in R-NPs, but in a lower proportion, even though it could be considered developing selective chemical treatment in future works, in order to enhance the biomolecules attachment over the R-NPs. But at the moment, this work focused on the $\mathrm{SiO}_{2}$ surface modification methods.

The optical interrogation of $\mathrm{SiO}_{2}$ surfaces was accomplished from above, with the surfaces in dry conditions and with the optical set-up from the GOPB. 
Under vertical and optical interrogation with the optical set-up, the interferometers of $660 \mathrm{~nm}$ of $\mathrm{SiO}_{2}$ produce several resonances in the studied wavelength range, (visible range). For the biofunctionalization assessment it was studied the position shift of the minimum resonance centered at $760 \mathrm{~nm}$. Different cells were drawn over the surface, to facilitate the positioning of the bundle fiber along the chip surface.

\subsubsection{Biofunctionalization of $\mathrm{SiO}_{2}$ with GPTMS}

3-Glycidyloxypropyl trimethoxysilane is a commonly used cross-linker between silicon based inorganic surfaces and organic molecules. [Li, 2014]. It has three methoxy groups $\left(-\mathrm{OCH}_{3}\right)$ acting as the binding sites to the inorganic substrate, and a functional group formed by an epoxy group available for the biomolecules binding, similar to the epoxy groups present in epoxy polymers such SU-8 resist. This silane was tested with the IgG/anti-IgG above-mentioned bioapplication.

Interferometer used, consisted on a $10 \times 10 \mathrm{~mm}^{2} \mathrm{SiO}_{2}$ surface of $660 \mathrm{~nm}$ thick, over amorphous silicon. It was treated with GPTMS and subsequently IgG was incubated overnight. The biofunctionalization protocol is described in figure 85 .

The surface samples were cleaned by sonication with acetone and $\mathrm{Di}-\mathrm{H}_{2} \mathrm{O}$ during five minutes subsequently. Then, surface was activated through hot-piranha solution $\left(\mathrm{H}_{2} \mathrm{SO}_{4}: \mathrm{H}_{2} \mathrm{O}_{2}-3: 1\right)$ during one hour. For the silanization it was used a solution of GPTMS pure, purchased from Sigma Aldrich. The reaction occurred over night, and then the surface was gently rinsed with ethanol, in order to remove physisorbed material, and dried with clean air for the incubation of the IgG (50 $\mu \mathrm{g} / \mathrm{mL}$ in $\mathrm{PBS}$ ) at $37^{\circ} \mathrm{C}$ in humidity conditions to prevent evaporation, all night long, and then washed with pure water and PBS-T as explained. After this process, the $\mathrm{SiO}_{2}$ surface was apparently properly biofunctionalized and apriory ready for the recognition of the anti-IgG. 


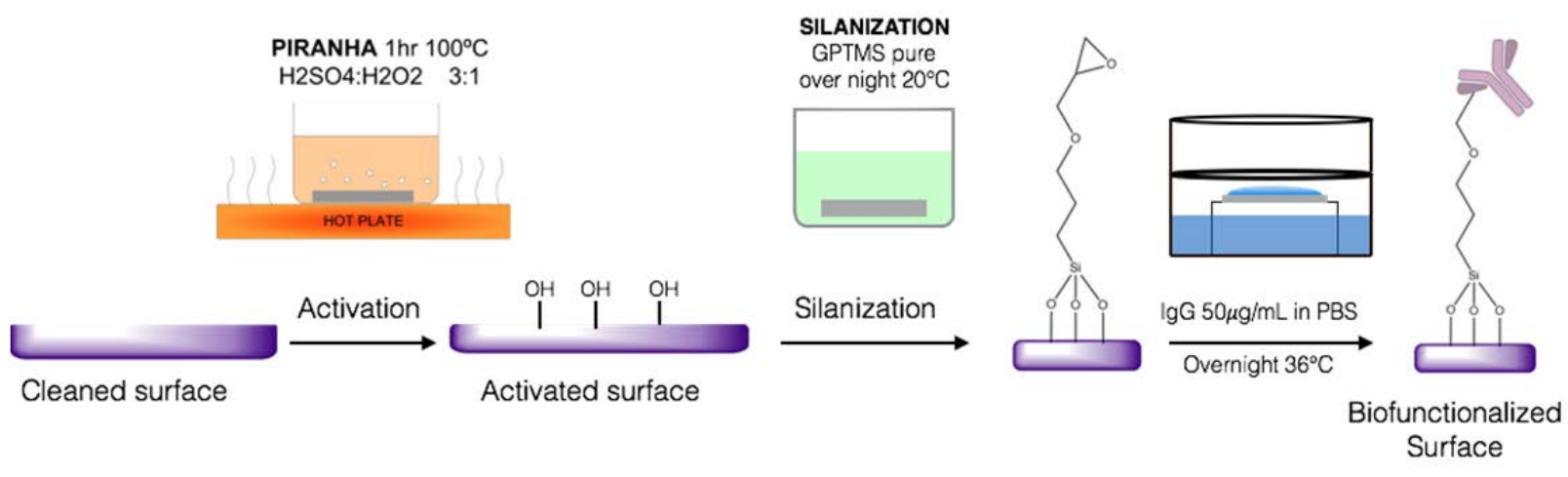

Figure 85: Biofunctionalization process of $\mathrm{SiO}_{2}$ flat interferometer with GPTMS.

\subsubsection{Biofunctionalization of $\mathrm{SiO}_{2}$ with APTMS}

For biofunctionalization with APTMS, $\mathrm{SiO}_{2}$ surface was cleaned in four different steps. Five minutes sonication with $20 \%$ SDS (sodium dodecyl sulphate) in water, followed by 5 minutes sonication of ethanol, acetone and pure water consecutively in that order. After works, surface was activated with Piranha solution and immersed in 5\% of APTMS in anhydrous ethanol overnight. The next morning sample was gently rinsed with ethanol and cured at $110^{\circ} \mathrm{C}$ in a hot-plate for one hour. Curing stage results primordial for the stabilization of the silane layer newly created.

As a coupling agent it was incubated $10 \%$ of GA in PBS during one hour. This bifunctional molecule binds on one side to the amine group of APTMS, leaving a free aldehyde group, for the binding of amine groups of the IgG during the immobilization. This last step was accomplished by incubating $\operatorname{IgG}(50 \mu \mathrm{g} / \mathbf{m L}$ in PBS) at $37^{\circ} \mathrm{C}$ in humidity all night long and washed with pure water and PBS-T. 


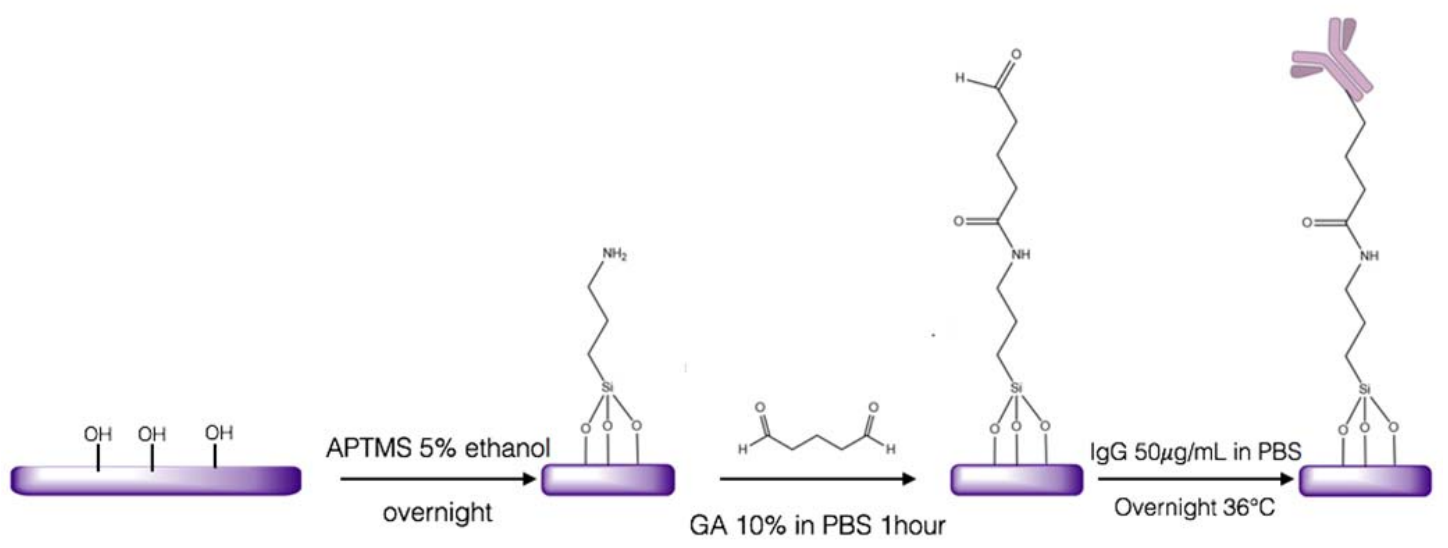

Figure 86: Biofunctionalization process of $\mathrm{SiO}_{2}$ flat interferometer with APTMS and GA.

8.1.2.3 Results and conclusion of biofunctionalization protocols

In the scatter plot there are shown the positions of the resonance after the different biological events with the two silanes tested.
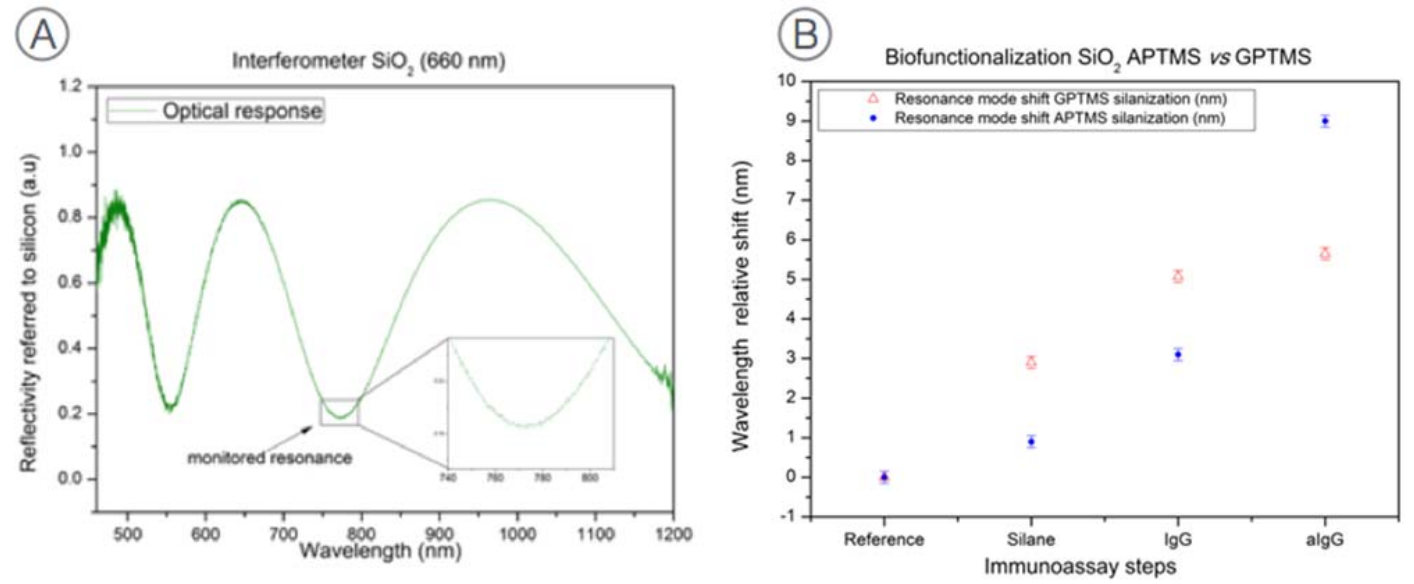

Figure 87: A. Optical response of the $660 \mathrm{~nm} \mathrm{SiO}_{2}$ interferometer. Resonance mode position shift for biofunctionalization with IgG and recognition af anti-IgG for both silanes tested: GPTMS and APTMS.

In figure 87.A it is showed the optical response of $\mathrm{SiO}_{2} 660 \mathrm{~nm}$, and it highlights the resonance mode monitored for both silane tests. In figure 87.B side it is showed a scatter plot that represent the position of the monitored resonance, for 
the different steps of the biofunctionalization protocols, with the two silanes and the IgG, and for the recognition of the anti-IgG.

After GPTMS silanization, the position of the resonance is shifted $2.9 \mathrm{~nm}$. Then the IgG attachment produced a resonance shift of $2.1 \mathrm{~nm}$ from GPTMS signal. It is not explained that a GPTMS monolayer produces a shift bigger than the IgG immobilization because the size of the IgG is approximately $12 \mathrm{~nm}$ and the GPTMS is around $1 \mathrm{~nm}$. This suggest that GPTMS may was polymerized creating a multilayer in which IgG had difficulties to attach orderly, and thus the shift caused by the immobilization of IgG was lower than expected. The recognition of the anti-IgG generated a shift of $0.52 \mathrm{~nm}$. Since IgG and anti-IgG are both antibodies, they have the same size and should provoke a similar wavelength shift. Thus the recognition was not successful, probably due to the rare IgG attachment in a probably undesirable position.

APTMS silanization provoked a shift of $0.9 \mathrm{~nm}$ and from there, the resonance shifted $2.8 \mathrm{~nm}$ for the IgG immobilization. Then the recognition of the anti-IgG produced a shift of $5 \mathrm{~nm}$ approximately. This suggests whether the IgG did not fully cover the surface with APTMS and thus there is some unspecific attachment of anti-IgG to the surface, or the agglomeration of the anti-IgG due to insufficient or improper washing process. Surface modification chemistry must be focused on the development of monolayer with good antifouling properties, preventing the unspecific attachment of the biomolecules.

APTMS silanization produced a shift of $0.9 \mathrm{~nm}$ and GPTMS $2.9 \mathrm{~nm}$. Since GPTMS almost triplicates the shift of the resonance found for APTMS, and according to the later biological measurements, it could be happening that the concentration of GPTMS for silanization was too high and therefore it was polymerized over the SiO2 surface, and a monolayer was not created.

The immobilization of IgG over both types of silanes produced a similar resonance shift. However recognition of anti-IgG with GPTMS silanization was barely observed. 
APTMS seemed more stable and controllable silane and apparently the use of GA enhances the IgG immobilization in a more organized way.

Thus, bearing in mind both type of silane tested results, it was decided to try APTMS biofunctionalization protocol for the biological sensing of R-NPs.

\subsection{Immunoassay over R-NPs in dry conditions}

After the results drawn from bulk sensing experiments, BICELLs based on R-NPs resulted in a promising and an interesting alternative for label-free detection of biomolecules. [Hernandez, 2016].

Thus, it was studied the capability of R-NPs to detect biological events through the binding of biomolecules. For that reason two different silanes were tested and finally APTMS was applied for the biofunctionalization protocol of the R-NPs arrays, now called BICELLs.

R-NPs used for this experiment were integrated in eight BICELLs of $1 \mathrm{~mm}^{2}$ on a quartz substrate of $14 \mathrm{~mm} \times 20 \mathrm{~mm}$ and $500 \mu \mathrm{m}$ thick. The lattice parameter used was $500 \mathrm{~nm}$ with Bragg reflectors and central cavity with the redesigned dimensions $\left(\mathrm{SiO}_{2} / \mathrm{Si}_{3} \mathrm{~N}_{4} 110\right.$ and $97 \mathrm{~nm}$ thick respectively and $200 \mathrm{~nm}$ thick for central cavity) (see chapter 7.5). The eight BICELLs were arranged into two columns and four rows providing an ideal layout for multiplexed label-free biosensors.

For the characterization of the BICELLs, the chip was placed on the holder fabricated ad-hoc with the R-NPs facing down, in contact with air (in dry conditions). Each BICELL was optically interrogated through the optical set-up with the spectrometer covering the visible range, where the resonance mode is formed, described in chapter 4 .

In order to assess the R-NPs biological response in was developed an immunoassay (IgG/anti-IgG) in dry conditions, over the eight different BICELLs. 
For the Biofunctionalization, it was carried out the same APTMS protocol before described for the $\mathrm{SiO}_{2}$ interferometer, but in this case for the eight different BICELLS.

Then, IgG was covalently immobilized over R-NPs and increasing concentrations of anti-IgG were incubated for the specific recognition, and measured seven repeated times, in order to obtain a curve sensing response, from which Limit of Detection of the R-NPs could be drawn.
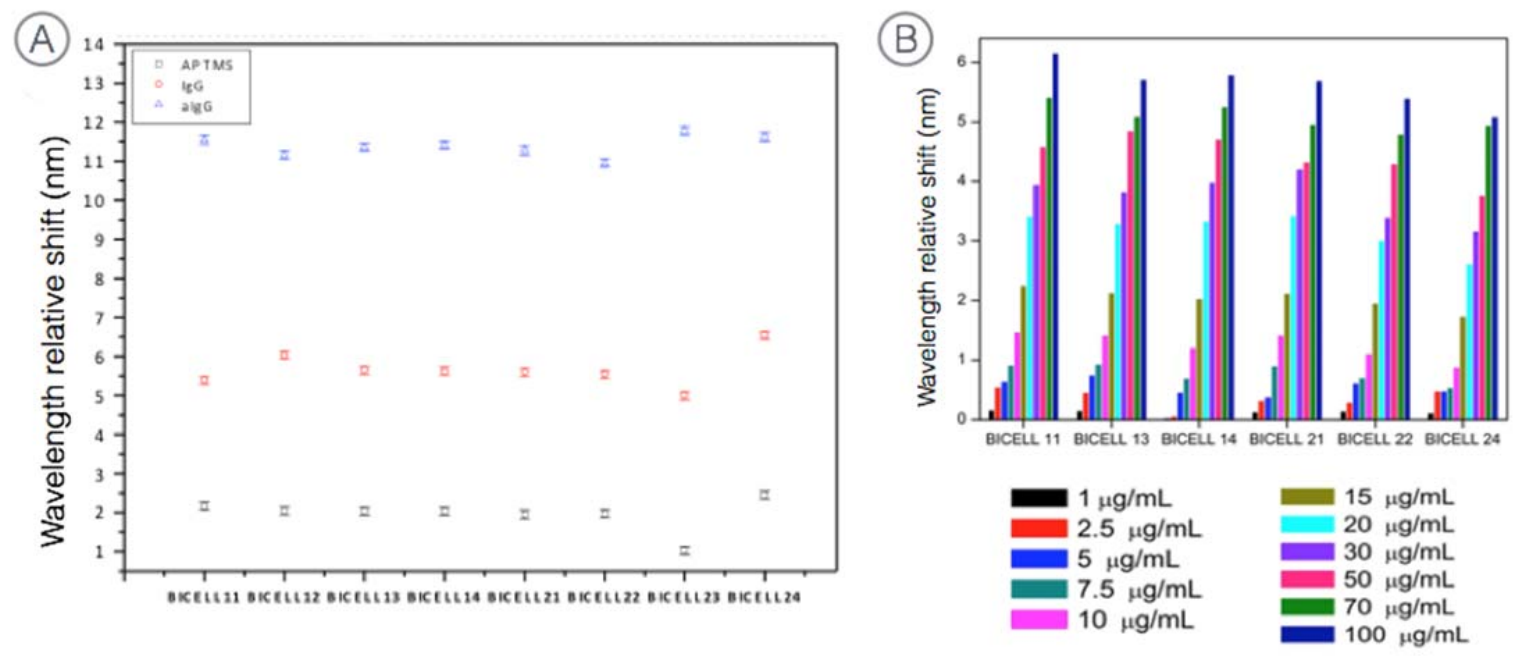

Figure 88: A. Resonance shift for differen events: APTMS surface modification, IgG incubation and anti-IgG recognition, for the eight BICELLS. B. Resonance shift for the different concentration of anti-IgG represented in coluns for the different BICELLS.

In figure 88, black squares represent the relative shift of the resonance mode after silanization in the eight BICELLs. It is around $2 \mathrm{~nm}$, which is slightly higher than expected. Bearing in mind that the IgG has a size of $12 \mathrm{~nm}$ and produced a shift of 5 $\mathrm{nm}$ approximately, it would be expected a resonance shift for APTMS which did not exceed $1 \mathrm{~nm}$. Probably the perfect monolayer was not created because APTMS underwent a polymerization process, since it is extremely complicated to keep anhydrous conditions during the whole process of silanization. Red dots show the relative shift after IgG immobilization $100 \mu \mathrm{g} / \mathrm{mL}$, and blue triangles represent the shift after recognition of anti-IgG $(100 \mu \mathrm{g} / \mathrm{mL})$ fully accomplished. The represented data correspond to the average of seven repeated measurements. The BICELLs are identified as a function of their coordinates in the chip (column, row). 
The molecules attachment during biofunctionalization protocol and the biomolecules binding in recognition events, provoke a surface thickness increment of the R-NP, thereby the resonance dip shifts to higher wavelength positions. This entails IgG/anti-IgG complex suppose a total shift of the resonance dip around 10nm. (Figure 88.A). Experimental data match to the theoretical calculation for this R-NPs.

Figure 88.B is a bar chart showing the increasing concentration of anti-IgG measured. $(1,2.5,5,7.5,10,15,20,30,50,70$, and $100 \mu \mathrm{g} / \mathrm{mL})$. These concentrations were incubated at $36^{\circ} \mathrm{C}$ for $30 \mathrm{~min}$ and washed with $\mathrm{Di}-\mathrm{H}_{2} \mathrm{O}$. After each incubation/washing step, the chip was dried and placed into the holder for monitoring the resonance position as a function of each of the concentrations mentioned. For each concentration step, each BICELL was measured seven times $(n=7)$ in order to determine the experimental uncertainty.

Notice that the response is similar for every BICELL with maximum dispersion of $1.7 \mathrm{~nm}$. It implies consistency of the fabrication process and robustness of the biofunctionalization and immunoassay results. Thus, R-NPs are suitable for multiplexing biosensors.

The recognition curve represents the relative wavelength increasing positions, as a function of anti-IgG concentration, fitting a sigmoidal function (figure 89.A). The first point of the curve corresponds to IgG immobilization and the last point is the anti-IgG $100 \mu \mathrm{g} / \mathrm{mL}$ from the BICELL with coordinates 21 . The optical spectra corresponding to these two points are shown in figure 89. B. The shift of the resonance can be noticed at once. 


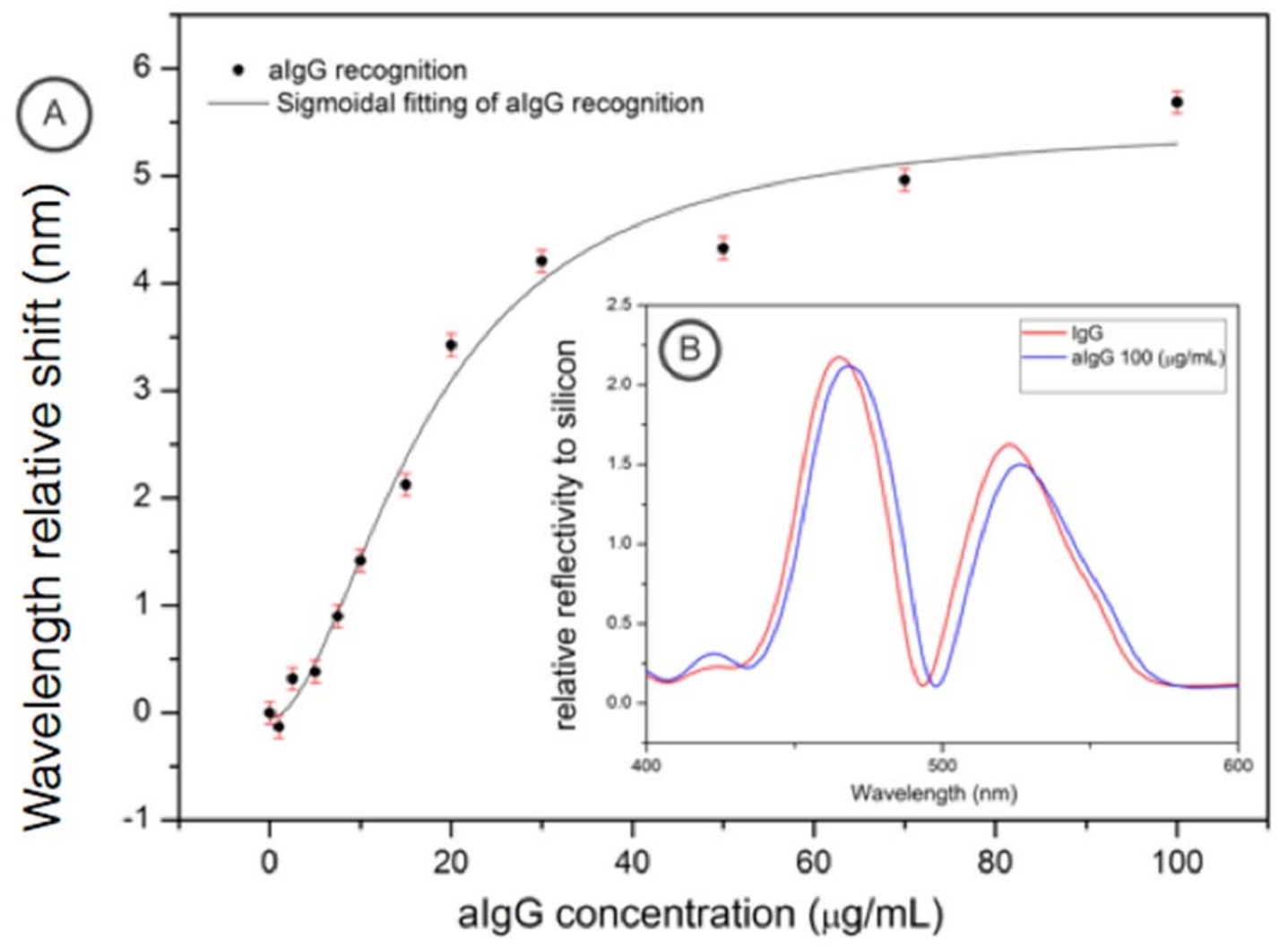

Figure 89: A. Recognition curve from anti-IgG on cell 21. B. Optical spectra of the cell with IgG (red) and shifted spectra from anti-IgG recognition (blue).

A sensitivity of $0.1716 \mathrm{~nm} / \mu \mathrm{g} \cdot \mathrm{mL}^{-1}$ was achieved for the BICELL 21 shown in figure 89 , considering a linear range from 0 to $20 \mu \mathrm{g} / \mathrm{mL}$.

Considering the resolution in wavelength as the main uncertainty source, which is $0.12 \mathrm{~nm}$, the experimental LoD obtained was $699 \mathrm{ng} / \mathrm{mL}$. However, in order to compare the performance of these transducers with others reported in the literature it must be used the same uncertainty value. For example, LoD reported for a resonant disk based on a slot waveguide was $1.66 \mathrm{ng} / \mathrm{mL}$ for anti-bovine serum albumin (BSA) detection [Barrios, 2008]. If applying the same U calculation method, by applying $1 \mathrm{pm}$ resolution, the LoD achieved in this experiment would be $5.82 \mathrm{ng} / \mathrm{mL}$, which is similar to that obtained in former works focused on nanopillars arrays as sensing structures for anti-BSA detection. 
Increasing the pillars aspect ratio and reducing the pitch, which is limited only by fabrication procedures, could improve these results. Increasing the RI contrast of the materials, and thus the reflectivity of the optical response, could also enhance the sensitivity of the R-NPs. For example, by using $\mathrm{Si} / \mathrm{SiO}_{2}$ Bragg reflectors. The biofunctionalization protocol seems to be adequate according to experimentaltheoretical results; nevertheless it could be optimized by preventing hydrolization, fouling, reducing times and simplifying the reactive handle.

\subsection{Immunoassay over R-NPs immersed in water}

Former immunoassay on the R-NPs was performed with the R-NPs in contact with air, in dry conditions. Nevertheless to observe the performance they would have in fluidic conditions, it was necessary to perform another immunoassay, in which the R-NPs would be in contact with the water. For that, the R-NPs chip was placed in the holder, filled with water with the pillars facing down and interrogated from the backside, which remains dry.

Besides, it was tested the susceptibility of R-NPs samples to be regenerated until bioreceptor level. In means that, after analyte detection, R-NPs undergo a regeneration process, which breaks the biorreceptor-analyte binding, which is based on electrostatic forces. After regeneration only remain the bioreceptor covalently attached to the surface, ready for the next sample detection. [Barrios, 2008].

For that purpose, after every step in the immunoassay, R-NPs were brought under clorhydric acid ( $\mathrm{HCl})$, for 10-20 seconds, then washed and measured in contact with water.

The procedure of the immunoassays carried out for this experiment, was the same as revealed in the former sub-chapter, but in this case, every time the R-NPs were interrogated, the holder was filled with $\mathrm{Di}-\mathrm{H}_{2} \mathrm{O}$. R-NPs fabrication parameters were also the same as in the former sub-chapter experiment. 
In figure 90, black triangles correspond to the R-NPs silanized with APTMS. It can be observed a resonance shift of $1.7 \mathrm{~nm}$ for the IgG immobilization. This matches to the theoretical calculations for the R-NPs immersed in water. Then, three different concentration of anti-IgG [5, 10 and $50 \mu \mathrm{g} / \mathrm{mL}$ ) were incubated and measured. It is observed a very shy shift for the anti-IgG recognition, even for the greatest concentrations, which does not even reach one $\mathrm{nm}(0.856 \mathrm{~nm})$. This could be due to an improper position of the IgG over the surface where anti-IgGs are unable to find the specific binding sites of IgG.

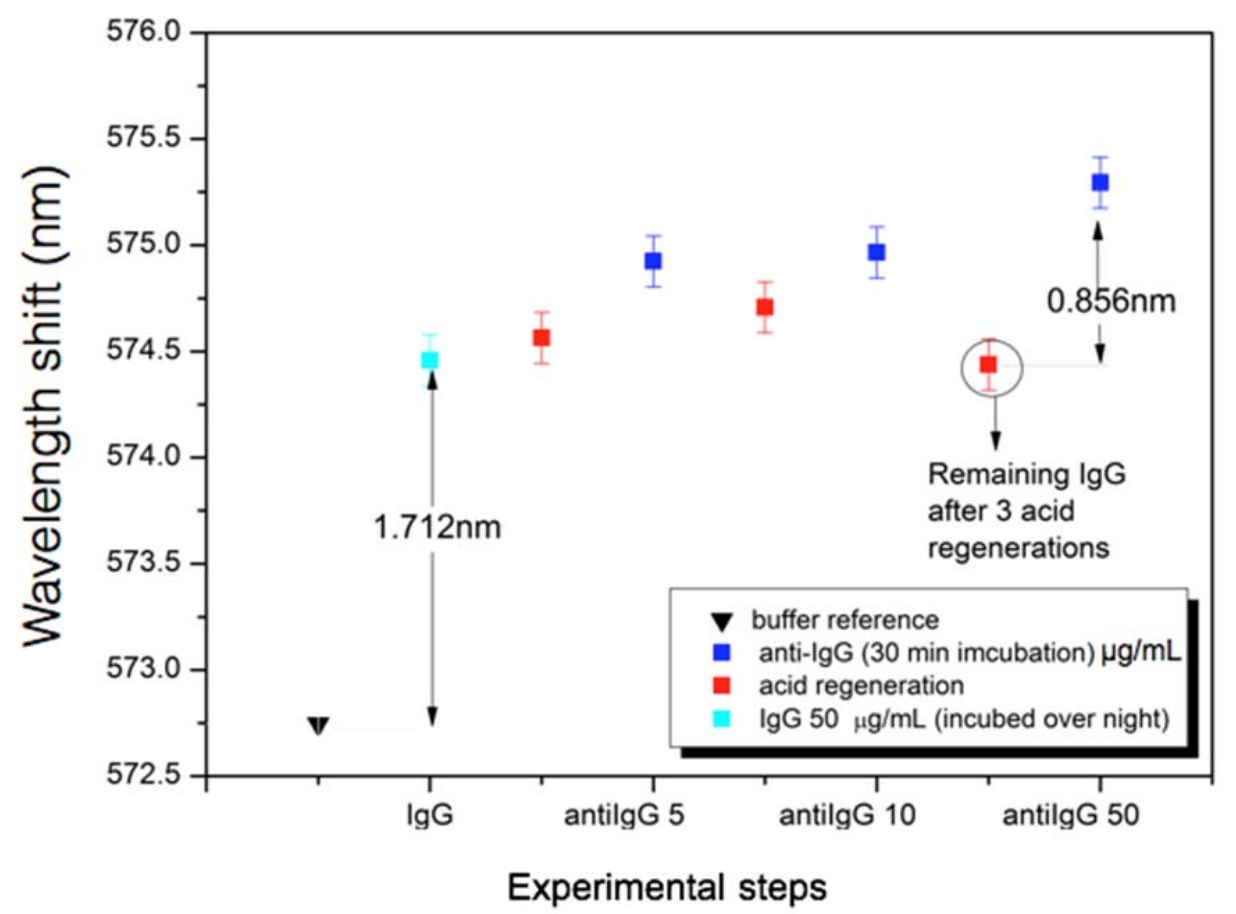

Figure 90: Immobilizationof IgG (light blue squares) and three different anti-IgG recognition concentrations after regerenation phase.

It is thus remarkable the difference on the shift provoked by the biomolecules, when R-NPs are immerse in water and when they are interrogated in dry conditions.

Red squares represent the regeneration phase accomplished after every biological event. The resonance approximately shifts to the position of the IgG immobilized, suggesting that regeneration process is able to undo the IgG/antilgG binding but not the IgG-surface binding. This implies that R-NPs could be used repeated times 
for biosensing purposes without needing to be biofunctionalized every single time and therefore they could be used for continuous monitoring in remote locations.

Nevertheless there could be a limit of regeneration cycles that IgG can stand. After that limit, the regeneration could break IgG-surface attachment. This limit should be explored in further works.

\subsection{Immobilization of R-NPs in continuous flow}

For the immobilization of the IgG in continuous flow, silanized R-NPs chip with APTMS was introduced in the flow cell of the fluidic system. R-NPs dimensions were the same as in the former experiment for R-NPs in fluidic conditions.

A solution of $100 \mu \mathrm{g} / \mathrm{mL}$ of IgG was pumped for more than two hours. One of the BICELLs was continuously interrogated with the optical set-up adjusted to the fluidic system and thus, the resonance position was monitored along the time, every 1.7 seconds. Pumping speed was $50 \mu \mathrm{L} / \mathrm{min}$, therefore the quantity of IgG used was more than $6 \mathrm{~mL}$. (Figure 91).

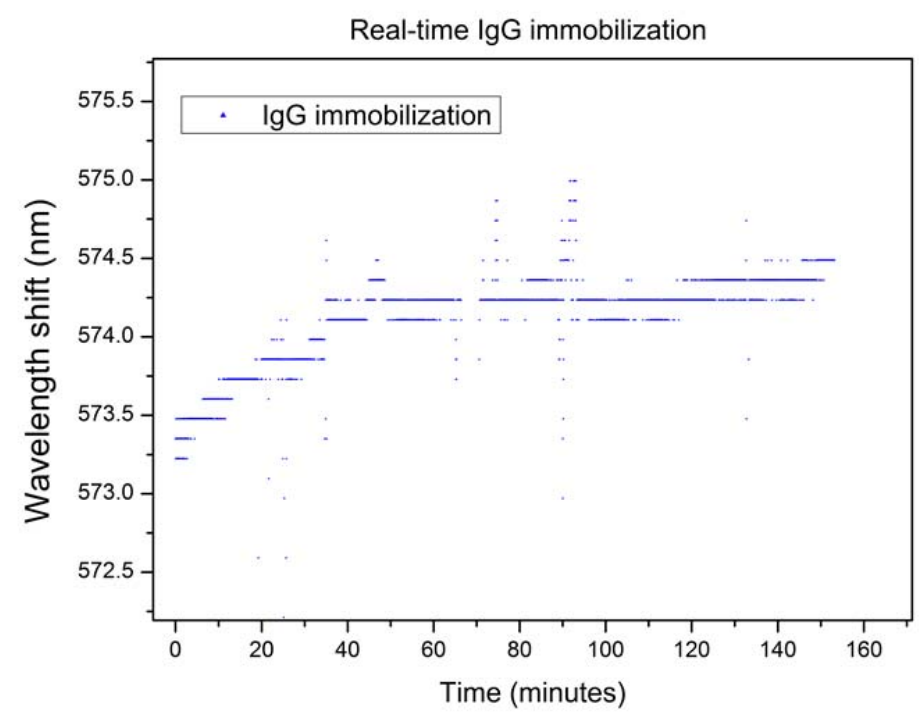

Figure 91: Real-time response for IgG immobilization in the fluidic system. 
After 50 minutes the position of the resonance is relatively stable. Blue dots out of the sigmoidal trend correspond to bubbles appearing inside the flow cell. The resonance shifts from $573.2 \mathrm{~nm}$ to $574.4 \mathrm{~nm}$, this is a relative shift of $1.2 \mathrm{~nm}$, which is in the order of what can be expected for this R-NPs being interrogated in fluidic conditions.

\subsection{Performance comparison}

\subsubsection{Immobilization of R-NPs: procedures comparison.}

For the interrogation of the R-NPs in contact with a fluid, immobilization was accomplished in two different forms: static immobilization (92.A) and continuous immobilization (92.B).

(A) Immobilization in static

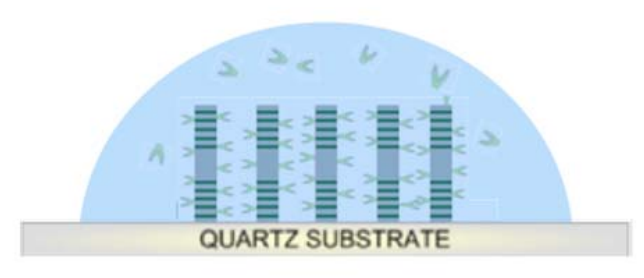

Figure 92: A. Immobilization of IgG in static (over night). B Immobilization of IgG in continuous flow.

The resonance shift is $1.7 \mathrm{~nm}$ for immobilization of IgG in static conditions and 1.2 $\mathrm{nm}$ in continuous flow system. The difference of $0.5 \mathrm{~nm}$ is not significant in this case and in both situations it could be claimed that biofunctionalization has been successfully accomplished.

Nevertheless, there are other influent parameters that will determine the adequation of one operating process or another. On one hand continuous 
immobilization takes less than one hour, in contrast to static immobilization where IgG must be incubated overnight. The reason of that is the dynamics of the biomolecules, which in continuous flow system they are in continuous movement and the chances of taking contact with the surface are bigger.

Although, in the other hand, for the static immobilization, the IgG quantity used was $50 \mu \mathrm{L}$. Unlike this, in the continuous flow immobilization, more than $6 \mathrm{~mL}$ were used. Bearing in mind that after one hour the surface was already immobilized with IgG, the quantity used at that point would be $3 \mathrm{~mL}$ of IgG. This means that in continuous flow, the quantity of IgG used was sixty times more than quantity used for static immobilization. In addition, selective biofunctionalization of different bioreceptors in the different BICELLs, for multiplexing purposes, cannot be performed in the continuous flow system.

Which method to use for IgG immobilization is a matter of resources applied vs time consumed.

It would be desirable to develop a and continuous fluidic system incorporating a fluidic chip with smaller channels and small section tubing, with short distances from reservoir to flow cell, in which small volumes of biomolecules could be pumped so not such a big sample quantity would be applied.

8.5.2 Biosensing performance of R-NPs: under dry condition or immersed in water

In chapter 7 it was demonstrated the feasibility of using R-NPs for detecting small changes of the RI media surrounding them. Then, in the former subchapter it has been revealed that R-NPs are able to detect such a small changes in RI as those provoked by the biomolecule attachment and interactions. In addiction it was stated that they are adequate to be used as label-free multiplexed biosensor.

The biological events produce shifts in resonance modes suitable to be monitored along the immobilization of bioreceptors, or during the specific recognition of 
target biomolecules. Those shifts are not equal when R-NPs are interrogated in dry conditions or in contact with a buffer, as it occurs in the continuous flow real-time experiments.

Thus, the purpose of this experiment was to demonstrate through a biosensing experiment, the increased performance of R-NPs when they are vertically interrogated in dry conditions. Thus, an IgG/anti-IgG immunoassay was developed over R-NPs. Every step of the immunoassay was characterized within the two comparing methodologies: on one hand, by immersing the pillars in water ffigure 93. A) and on the other hand, with the pillars in dry conditions (figure 93.B).
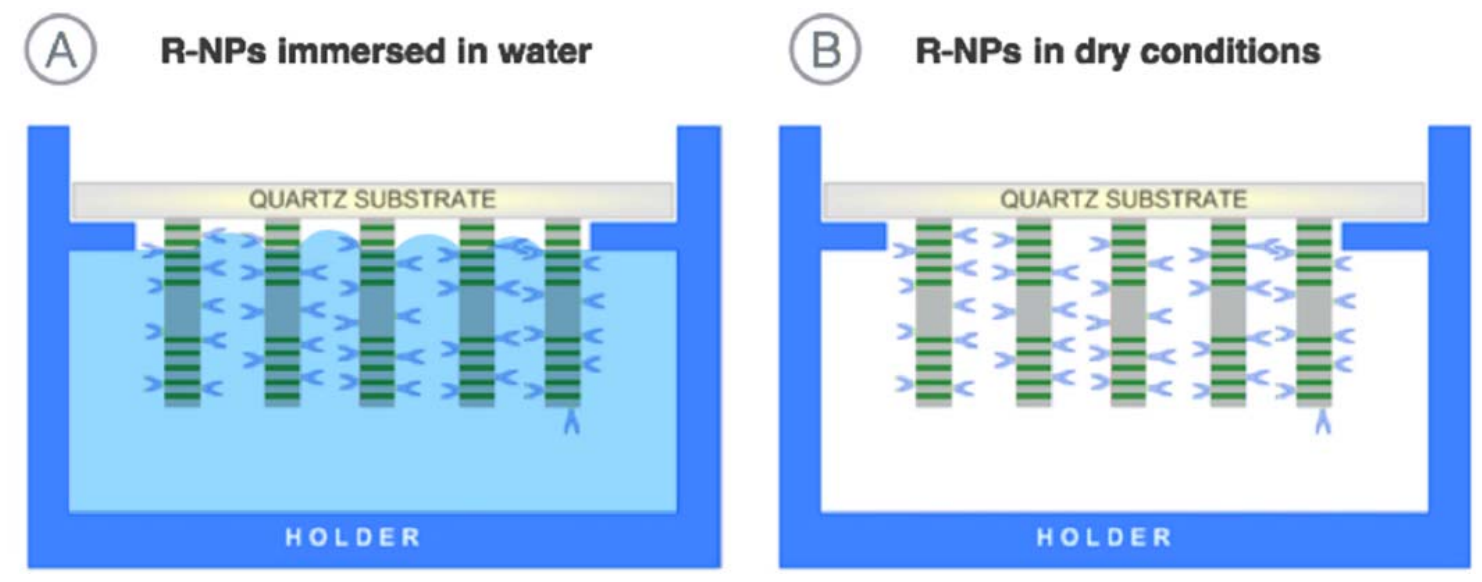

Figure 93: A. Scheme of R-NPs immersed in water. B Scheme of R-NPs in dry conditions.

For that reason, the same R-NPs dimensions sample, used for the former R-NPs biosensor experiment (500 nm pitch), was located in the holder fabricated ad-hoc with the pillars facing down, as described in previous sections. After every sensing step, the arrays were independently, vertically interrogated with the bundle of fiber with a beam spot size of $500 \mu \mathrm{m}$ diameter, with the spectrometer (with a resolution of $0.1 \mathrm{~nm}$ ), covering the visible range in which the resonance mode can be observed.

Firstly, BICELLs were vertically interrogated without any previous treatment in order to have a reference signal of the spectral mode for both characterization procedures: with pillars immersed in water and with pillars in contact with air. 
Also, there were performed theoretical calculations for the R-NPs spectra using the commercial software RSoft, and in particular its feature for Finite Difference Time Domain calculations Fullwave. There were obtained the reflectivity referred to silicon of the photonic structure for both dry and immersed in water conditions.
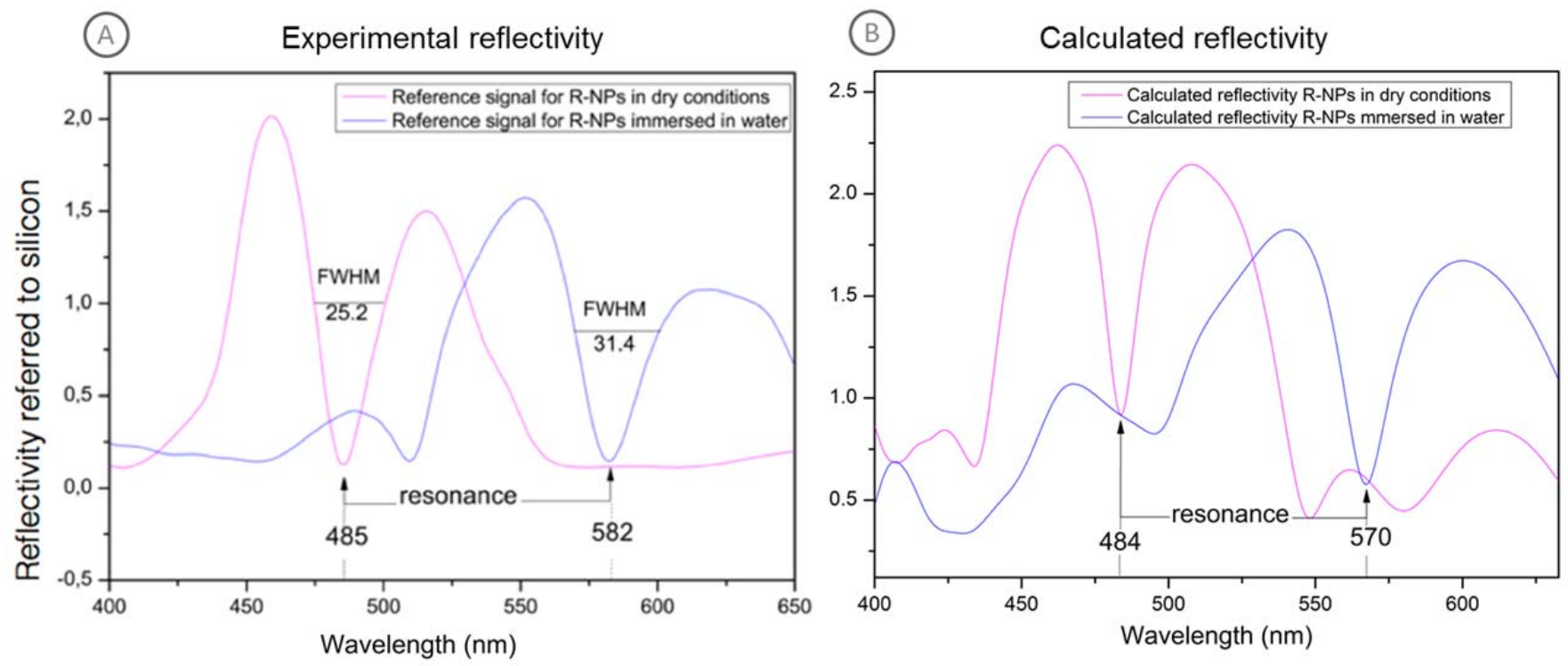

Figure 94: A. Experimental transducing signal for different characterization modes: Pink spectra: R-NPs arrays response in dry conditions. Blue spectra: water immersed RNPs arrays response. B. Calculated transducing signal of the R-NPs in dry conditions (pink line) and immersed in water (blue line).

In figure 94.A, the pink line represents the reflectance signal as a function of the wavelength when the R-NPs are dry. The Blue spectrum corresponds to the R-NPs immersed in water. It can be observed a smaller intensity of the transducing signal in liquid infiltrated R-NPs, which barely reaches 1.5 relative units of the reflectance referred to silicon. Besides, the resonant mode is centered at $582 \mathrm{~nm}$, in comparison, when the pillars are in dry conditions; it is centered at $485 \mathrm{~nm}$.

Figure 94.B shows the reflectivity spectra calculated using 3D-FDTD algorithm, without considering an attached biofilm layer. In the case of dry conditions this mode is centered around $484 \mathrm{~nm}$ matching to the experimental response, whereas for water conditions the dip is around $570 \mathrm{~nm}$; which is shifted from the experimental resonance mode obserbed (around 582nm). This is probably due to 
an inaccuracy in the calculation of the effective refractive indexes, for this photinic structure in fluid conditions.

Two other parameters may be mentioned although, in this case they are not decisive to determine biosensor performance: the signal-to-noise ratio (SNR) and the width of the resonance dip, expressed as the Full Wave at Half Maximum (FWHM). Both parameters provide information about the biosensor yield [de Vicente, 2014]. For the same transducer, a higher SNR ratio and a smaller FWHM are observed for the R-NPs interrogated in dry conditions.

Secondly, for the immunoassay over the R-NPs, they were firstly biofunctionalized with the IgG. For that reason, it was developed a silanization protocol based on 3aminopropyl trimethoxysilane (APTMS), and glutaraldehyde (GA) as linker to the amine groups of the antibody, which is further described in our previous work [Hernández, 2016]. The immobilization of the IgG over R-NPs was carried out through the incubation of a drop of $50 \mu \mathrm{g} / \mathrm{mL}$ of IgG, over night and in humid conditions in order to avoid the evaporation of the sample drop. Subsequently the chip with the R-NPs was rinsed with PBS-tween and water and properly dried with clean air. Then the chip was placed on the empty holder (with the R-NPs facing down) for the interrogation of the R-NPs in dry conditions and later on, the holder was filled with water for the interrogation of the R-NPs in humid conditions. This process of incubation, rinsing, drying and both methods of interrogation, (in dry conditions and with the R-NPs immersed in water), was repeated for the recognition of increasing concentrations of anti-IgG with no regeneration phase between them. The incubation time of the increasing concentrations of anti-IgG was 30 minutes. 
Moreover, the relative shift of the resonant deep during the silanization process and during biofunctionalization (IgG immobilization), for both measuring typologies, is represented in figure 95.

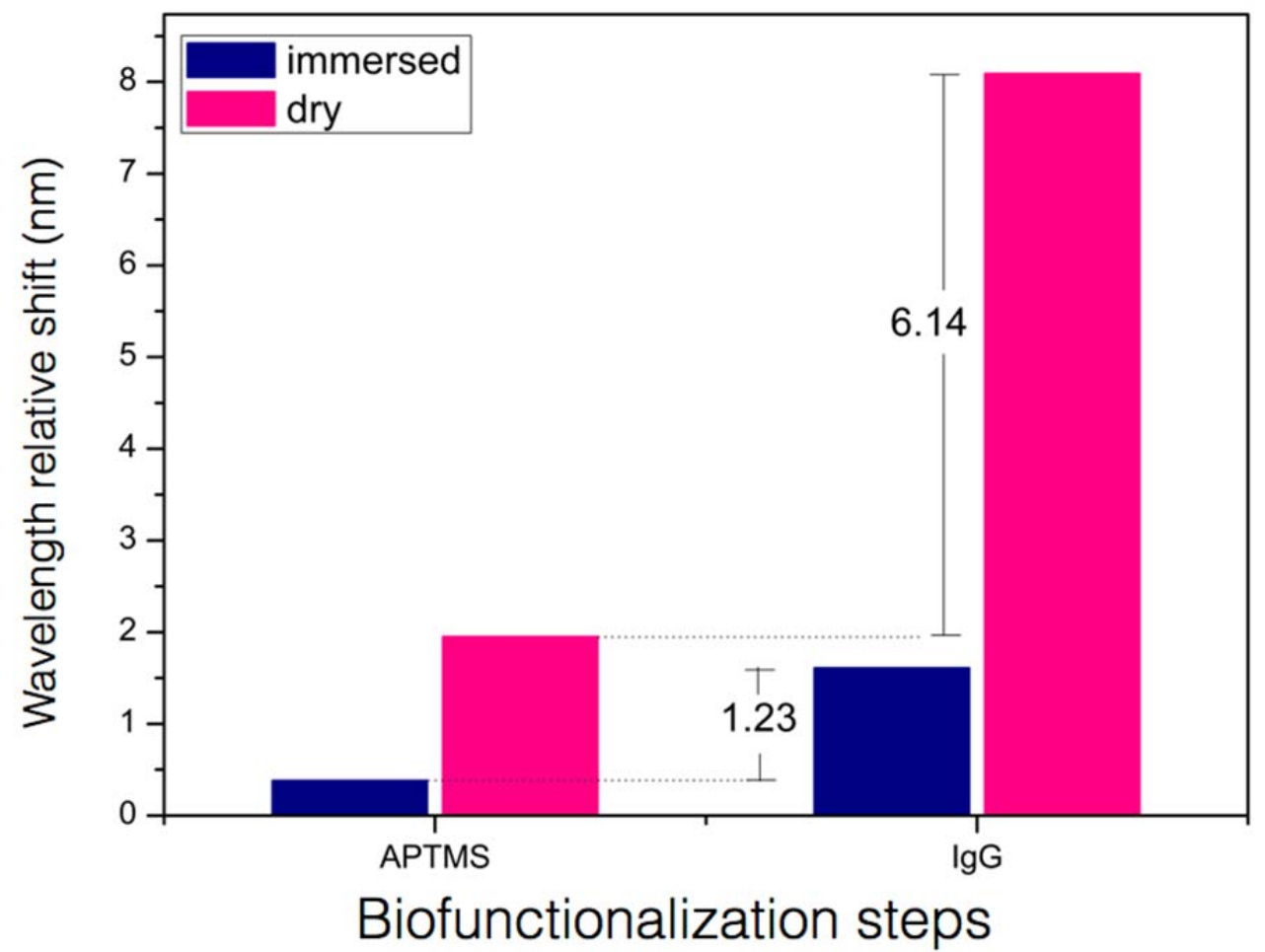

Figure 95: Immobilization steps as a function of relative shift of the resonant mode for arrays immersed (blue bars) and not immersed (pink bars).

The pink bars show the shift of the R-NPs resonance position after every biofunctionalization step, when the R-NPs are dry. The shift is around $2.2 \mathrm{~nm}$ after APTMS silanization and $6.14 \mathrm{~nm}$ for the IgG immobilization over the silane layer. For the same R-NPs immersed in water (blue bars) the shift is $0.4 \mathrm{~nm}$ for the APTMS and $1.23 \mathrm{~nm}$ for the IgG attachment. These results do not imply that the amount of antibody attached on the surface of the pillars is lower for R-NPs immersed, because it is the same R-NPs sample. However, they reveal that the changes occurring over biosensing structures, are more easily observed when R-NPs are not immersed. The shift corresponding to the IgG immobilization is approximately 3 times the shift of the silane layer formation. 
As summary, it can be stated that higher wavelength displacement results in greater sensitivity $(m)$. Also, due to the lower FWHM and higher SNR, the uncertainty $(u)$ on the calculation of the minimum position of the resonance, is smaller for R-NPs interrogated in dry conditions.

Thus, due to these figures, it was expected achieving a better performance in terms of limit of detection $(L o D)$ under dry conditions. In order to demonstrate and check these abovementioned assumptions, it was carried out a recognition experiment to obtain the biosensing curve response. (Figure 96.A). For this recognition phase, different concentrations of anti-IgG were incubated in the R-NPs BICELLs. The point in 0 represents the stable biolayer of $\operatorname{IgG}$ and the subsequent points correspond to the relative shift for the increasing concentrations of anti-IgG $(2.5,5$, 10, 15, 20 and $50 \mu \mathrm{g} / \mathrm{mL}$ ). Figures $96 . \mathrm{B}$ and D correspond to the transducing signal of the R-NPs for the IgG is immobilization and for the recognition of anti-IgG at a concentration of $50 \mu \mathrm{g} / \mathrm{mL}$, assuming that the surface is saturated at that concentration. It can be seen the higher resonant mode shift for interrogation of R-NPs in dry conditions. 

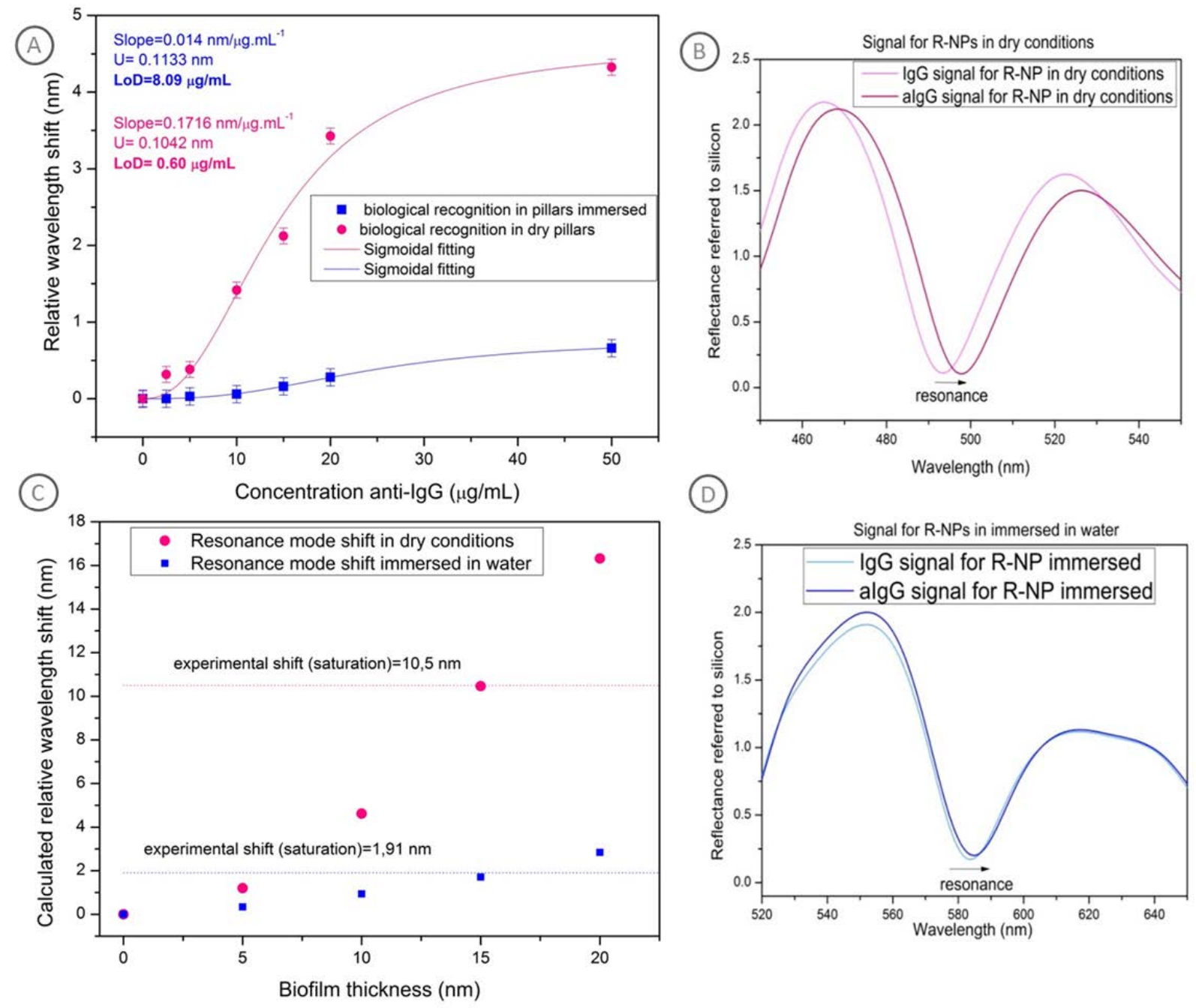

Figure 96: A. Recognition curve of anti-IgG of one of the BICELLS. R-NPs in dry conditions (pink dots). R-NPs immersed in water (blue squares). B. Bulk signal shift for anti-IgG recognition in dry conditions. C. Calculated shif of the resonant mode as a function of a bilofilm thickness. $D$. Bulk signal shift for anti-IgG recognition with the R-NPs immersed.

The relative shift of the highest concentration of anti-IgG recognition for the dry R-NPs, is $4.36 \mathrm{~nm}$ and for the immersed pillars is $0.68 \mathrm{~nm}$. Knowing the IgG and anti-IgG biomolecules have the same size (around $12 \mathrm{~nm}$ ), it would be expected a similar shift for the recognition of the anti-IgG, than for the immobilization of IgG (6.14 and 1.23 for dry and fluidic conditions respectively) if the yield of the recognition was $100 \%$. Therefore it is assumed that probably the orientation of the IgG was not the ideal, and consequently there were some fragment antigen areas (Fab), which were not available for the recognition process. 
Besides, it was calculated the theoretical shift of the resonance mode when adding a biofilm layer (with a refractive index of 1.41 surrounding the pillars) of 5, 10, 15 and $20 \mathrm{~nm}$ (Figure 96.C), to the photonic structure for both fluid and dry conditions. For the calculations there were considered three-dimensional space domain, with a grid resolution varying from $20 \mathrm{~nm}$ for the bulk pillars, to $5 \mathrm{~nm}$ for the biofilm layer.

The total shift, considering $20 \mathrm{~nm}$ of biofilm was of $2.84 \mathrm{~nm}$ (immersed in water) and $16.32 \mathrm{~nm}$ (dry conditions); more than 5 times (5.75) higher for dry conditions. The maximum shift reached experimentally was of $2.31 \mathrm{~nm}$ (water) and $12.7 \mathrm{~nm}$ (dry), considering the biofunctionalization of IgG plus the anti-IgG recognition shift; 5.5 times higher for dry conditions in this case. Comparing experimental and theoretical data, it was observed, that the saturation shift for both conditions (dry and immersed) seems to correspond with a biofilm thickness around 17-18 nm. This is in accordance with the relatively low shift for the biorecognition process, and thus a bit lower than expected (a biofilm layer of 20-25 nm was expected for this calculations, bearing in mind that the IgG and anti-IgG size is $12 \mathrm{~nm}$ ).

As it could be inferred from the resonant modes in figure 96.A, the standard uncertainty for the minimums identification, is higher when R-NPs are measured in wet conditions, due to a lower SNR and a higher FWHM. Therefore the expanded uncertainty $\mathrm{U}$ is also higher for the immersed R-NPs.

Considering $20 \mu \mathrm{g} / \mathrm{mL}$ as the saturation point it was observed that the sensitivity $(m)$, when the R-NPs are dry, is one order of magnitude higher regarding wet pillars (0.1716 and $0.014 \mathrm{~nm} / \mu \mathrm{g} \cdot \mathrm{mL}^{-1}$ respectively). Thus, sensitivity, which is the resonance shift as a function of the analyte concentration, has a greater influence on the LoD compared to the influence of the uncertainty, which was very similar for both cases of study.

In accordance with uncertainty and sensitivity values, the LoD, expressed in equation 7 , is improved 13.2 times if the experiment is performed with the pillars 
in dry conditions. (8.08 vs $0.6 \mathrm{~g} / \mathrm{mL}$ ). Table 5 summearizes the results obtained in the experiment.

Table 5: Comparison of the figures of merit observed for R-NPs as biological sensors immersed in water and in dry conditions

\begin{tabular}{|l|c|c|c|c|}
\hline & $\begin{array}{c}\text { FWHM } \\
\mathrm{nm}\end{array}$ & $\begin{array}{c}\text { Uncertainty (U) } \\
\mathrm{nm}\end{array}$ & $\begin{array}{c}\text { Sensitivity (m) } \\
\mathrm{nm} / \mu \mathrm{g}^{*} \mathrm{~mL}^{-1}\end{array}$ & $\begin{array}{c}\text { Limit of Detection } \\
(\mathrm{LoD}) \boldsymbol{\mu g} / \mathrm{mL}\end{array}$ \\
\hline $\begin{array}{l}\text { R-NPs } \\
\text { immersed in } \\
\text { water in dry }\end{array}$ & 31.41 & 0.11 & 0.01 & 8.08 \\
\hline $\begin{array}{l}\text { R-NPs in } \\
\text { conditions }\end{array}$ & 25.20 & 0.10 & 0.17 & 0.60 \\
\hline
\end{tabular}

This improvement in dry conditions is mainly due to the higher shift of the resonance mode, induced by the higher Refractive Index Contrast $(\Delta n)$ between R-NPs and air.

$\Delta n$ between R-NPs $(n=1.8)$ and water $(n=1.33)$ is lower than the $\Delta$ n existing between R-NPs and air $(n=1)$. In fact, the sensitivity of the detection in dry conditions comes from the higher refractive index contrast between the surrounding media and the bioanalyte layer. In wet conditions, the index of the surrounding media is 1.33 , and 1.41 for the biolayer, whereas in dry conditions the index of the surrounding media is 1 . The change of the effective refractive index of the surrounding media when introducing a biolayer over the pillars is higher in the case of dry conditions, due to the higher $\Delta n$ between indexes of air and biofilm, compared to $\Delta n$ between water and biofilm. This higher contrast in RI, results in higher values of the resonance shift, as seen experimentally.

In this work it was evidenced the result of measuring the same sample, with the same measuring system but in two different media: water and air. It was demonstrated the benefits of measuring R-NPs in dry conditions, where a reduction of the limit of detection in more than an order of magnitude is reached; therefore, this is a first approach of R-NPs to Point of Care devices for in-situ diagnosis. However in order to truly achieve a high-quality commercial biosensor based on R-NPs, work on this new generation of transducer must continue. 


\subsection{Surface modification techniques developed in Wageningen}

At the beginning of the year 2017, during the months of January, February and March, there was developed other biofunctionalization protocol at the Department of Organic Chemistry (ORC) of the Wageningen University (WUR).

The international research stay was focused on surface modification strategies, in order to optimize the biofunctionalization process, and thus try to maximize the biosensor performance.

At ORC of WUR there were used flat $\mathrm{SiO}_{2}$ surfaces chips $\left(1 \mathrm{~cm}^{2}\right)$, to be able to perform different surface analysis techniques. Then, an effective protocol of surface modification for biomolecules attachment on $\mathrm{SiO}_{2}$ surfaces would be transferred to R-NPs.

Analysis techniques used during the stay were:

- X ray photoelectron spectroscopy XPS: This technique has been historically used for surface analysis since at least second part of last century. It consists on an X-ray source irradiating the surface, and an electron energy analyzer, to detect the energy of the photoemited electrons on the surface, provoked by the X-ray. The process needs to take place in a high vacuum, so electrons can reach the detector without being scattered by residual gas molecules [Feldman, 1986]. The goal of XPS is the determination of the elemental composition of the outermost atomic layer (10 nm approximately) and, characterization of binding states. Binding energy ( $E$ binding) is calculated form the Rutherfor equation:

$$
\text { E binding }=E \text { photon }-(\text { E kinetic }+\varnothing) \quad(\text { Equation } 20)
$$

Where $E$ photon is the energy of the X-ray source, $E$ kinetic is the energy of electrons measured and $\varnothing$ is work function. 
Thicknesses of the organic layers ( $t$ ), from XPS results were calculated by applying the next equation:

$$
t=-\lambda \sin \theta \ln \left(I / I_{0}\right)
$$

Where, $I_{o}$ is the intensity of the signal of XPS ( $\left.\mathrm{Si} 2 \mathrm{p}\right)$ for the $\mathrm{SiO}_{2}$ blank sample. $I$, is the XPS signal intensity of the modified or biofunctionalized $\mathrm{SiO}_{2}$ surface. $\theta$ is the photoelectron emission takeoff angle $\left(80^{\circ}\right)$ and $\lambda$ is the attenuation length of the electrons in the organic layer which depends on the kinetic energy $(K E)$ of the electron being ejected; in this case the attenuation length was calculated by using Laibinis et al., formula $[\lambda(K E)=9+0.022 K E]$, resulting in a value of $3.95 \mathrm{~nm}$. [Dorvel, 2010] [Laibinis, 1991].

XPS analyses were performed using a JPS-9200 (Joel, Japan) photoelectron spectrometer, using a monochromatic AL X-ray radiation source at $12 \mathrm{kV}$ and $20 \mathrm{~mA}$. Data were then processed using CASA peak fitting program.

- Ellyspsometry: It is an optical analysis technique that is used for the determination of the RI and the thickness of thin films of dielectric material. It was used a rotating Sentech Instruments (Type SE-400) ellipsometer, operating at $632.8 \mathrm{~nm}$ (He-Ne laser), and an angle of incidence of $70^{\circ}$.

- Scanning Contact Angle (SCA): This technique measures the angle formed by a water drop and the studied surface. Low contact angle indicates highly hydrophilic surface, and high contact angle suggest low hydrophicity. The equipment used was a DSA-100 (Krüs, Germany). [CSC. Scientific Company].

- Atomic Force Microscope (AFM): This equipment uses a prove tip couple to a microscopic lever, to study the topography of a sample. Images were 
taken using a JSPM-5400 Scanning Probe Microscope (Jeol, Japan) with silicon cantilevers [Hilal, 2015].

- Fluorescence Microscope: It identifies single molecules with are labeled with a dye by illuminating the sampling area with a specific wavelength according to the dye wavelength absorbance.

First experiments were focused on the application adequate silane for surface modification of $\mathrm{SiO}_{2}$ flat surfaces. Most commonly silane used for surface modification is APTMS with 3 binding sites to the surface and one amine functional group to attach to biomolecules [Gunda, 2014]. Nevertheless, as seen in former experiments, this silane hardly forms a perfect monolayer since it has a great tendency to polymerization, preventing homogeneity along the surface, and disturbing the availability of the amine functional group for biomolecules binding. After some research in the literature it was found a very similar silane, also with an amine functional group, (aminopropyl dimethylmetoxysilane, APDMS) but with one single binding site $\left(\mathrm{OCH}_{3}\right)$ to the surface, promising a more stable single monolayer [Dorvel, 2010]. In the next table there are shown some of the main differences experimentally observed between both silanes tested during the stay in WUR. 
Table 6: Surface analyses of both silane surface modifications: APTMS and APDMS

\begin{tabular}{|c|c|c|}
\hline & APTMS & APDMS \\
\hline Chemical structure & $\sum_{\mathrm{CH}_{3} \mathrm{O}-\mathrm{SH}_{3}}^{\mathrm{NH}_{2}}-\mathrm{OCH}_{3}$ & $\sum_{\mathrm{O} 3 \mathrm{CH}}^{\mathrm{NH} 2}$ \\
\hline Contact Angle & $52^{\circ}$ & $70^{\circ}$ \\
\hline $\begin{array}{l}\text { Thickness (nm) } \\
\text { obtained from } \\
\text { ellypsometry }\end{array}$ & 4.30 & 0.99 \\
\hline $\begin{array}{c}\text { Thickness }(\mathrm{nm}) \\
\text { obtained from XPS } \\
\text { results } \\
t=-\lambda \sin \theta \ln \left(I / I_{0}\right)\end{array}$ & 3.00 & 0.80 \\
\hline $\mathrm{C} / \mathrm{N}$ ratio & 7.18 & 4.55 \\
\hline
\end{tabular}

According to the thicknesses drawn from ellypsometer as from XPS data results, APDMS is able to create a better monolayer over $\mathrm{SiO}_{2}$ surfaces. APTMS $\mathrm{SiO}_{2}$ samples, par contrary show thicknesses, which do not match to the molecule real size (around $1 \mathrm{~nm}$ ) suggesting polymerization of the silanes over the surface. Besides de $\mathrm{C} / \mathrm{N}$ ratio for APTMS is 3:1 and unlike this, XPS $\mathrm{C} / \mathrm{N}$ results is 7.18, indicating polymerization due to the excess of carbon observed. Unlike this, APDMS $\mathrm{C} / \mathrm{N}$ ratio results (4.55) are within the expected $\mathrm{C} / \mathrm{N}$ ratio from the molecule structure (5). Besides, APTMS ellypsometry analyses, revealed a thickness of $4.30 \mathrm{~nm}$, in contrast to the $0.99 \mathrm{~nm}$ thick found for the APDMS, suggesting a good monolayer formation. The same steps were followed for the surface modification with both silanes. Protocol is described in figure 97. 
Bearing in mind the comparing results, it was decided to continue working with the APDMS silane. Besides, the protocol developed for APDMS surface modification (figure 97), resulted to be easily reproducible.
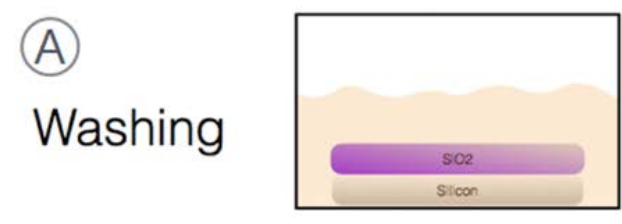

10 min sonication acetone

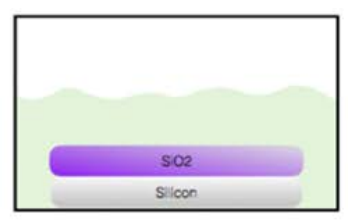

10 min sonication $\mathrm{Et}-\mathrm{OH}$

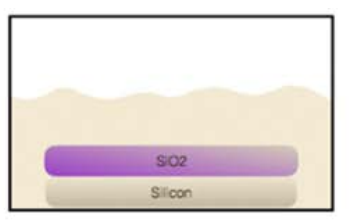

10 min sonication DCM

(B) Surface
Activation
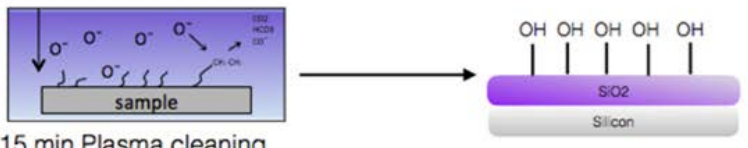

15 min Plasma cleaning

Silanization
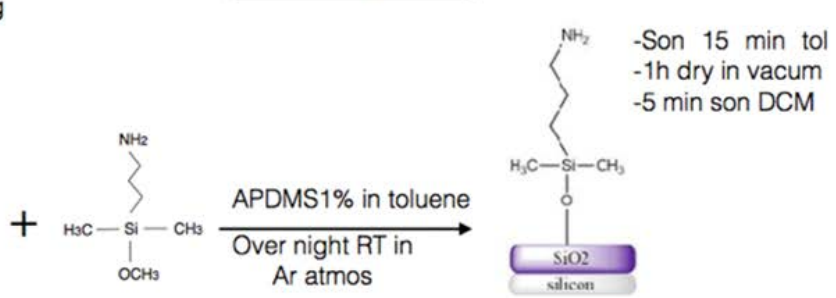

\section{Figure 97: Surface modification with APDMS. A. Washin steps, B. SUrface activation eoth plasma oxygen. C. Silanization with APDMS.}

The sample was firstly cleaned by sonication into different chemical reagent: acetone, ethanol and dicloromethane (DCM) (Figure 97. A). The surface was activated through the plasma oxygen to generate free $-\mathrm{OH}$ radicals to react with the metoxy group of APDMS (Figure 97. B). Finally samples were kept in a solution of $1 \%$ of APDMS in dry toluene overnight in an argon atmosphere. (Figure 97. C). The next day samples were sonicated to get them rid of the physisorbed materials and dried in vacuum at $80^{\circ} \mathrm{C}$ for 1 hour to settle silane. To develop this protocol there were performed several experiments with different solvents and times in order to try to optimize the process.

To verify the status of the APDMS monolayer formation, is was analyzed under several techniques: 

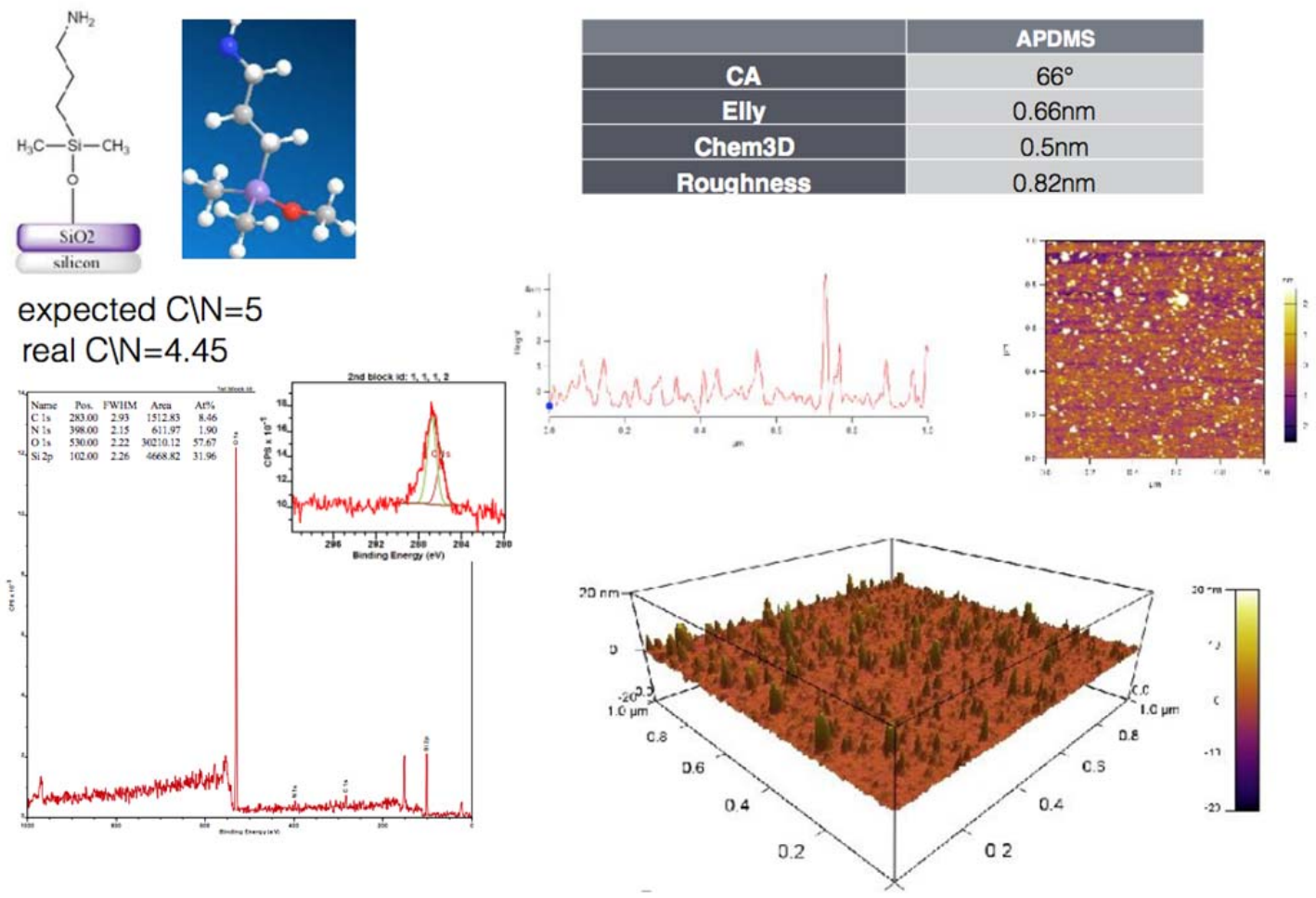

Figure 98: Analyses from $\mathrm{SiO}_{2}$ samples modified with APDMS.

Monolayer formation was successful and stable according to contact angle $66^{\circ}$ and ellypsometry $(0.66 \mathrm{~nm})$, matching the 3D chemical structure drawn from Chem Draw software. After activation with plasma oxygen, contact angle is practically null and is expected to increase over $50^{\circ}$ but lower than $70^{\circ}$ approximately in surface modifications strategies, looking for hydrophilic surfaces for biomolecules attachment. The C/N analytically obtained in XPS analyses (4.25) matched the theoretical chemical composition of APDMS (5). (Figure 98). AFM images show slightly increased roughness (regarding the blank samples which was around 0.5 $\mathrm{nm}$ ) and low density surfaces, indicating that the surface was indeed modified.

Next step to accomplish a successful biofunctionalization was the activation of the amine group remaining in the surface, through a coupling agent, in order to facilitate the attachment of the biomolecule.

To do this there are several activators commercially available that react with the amine group, such as disuccimidil carbonate (DSC), N-hydroxy succinimide (NHS), carbonyl diimidazol (CDI). [Sask, 2012], [Ling, 2006]. 
For this purpose it was used CDI due to the fact that this chemical was available at ORC-WUR facilities, and had been previously tested by them. The analysis performed after this step suggests that CDI reacted with the amine group remaining in the surface and that, it had not lose its nucleotide properties. For example if the amine is in contact with a media of a low $\mathrm{pH}$, it could be protonated and thus precipitated, losing its reacting properties. (Reaction 3).

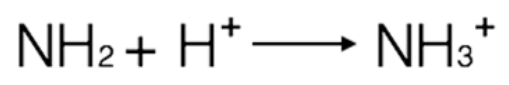

Reaction 3
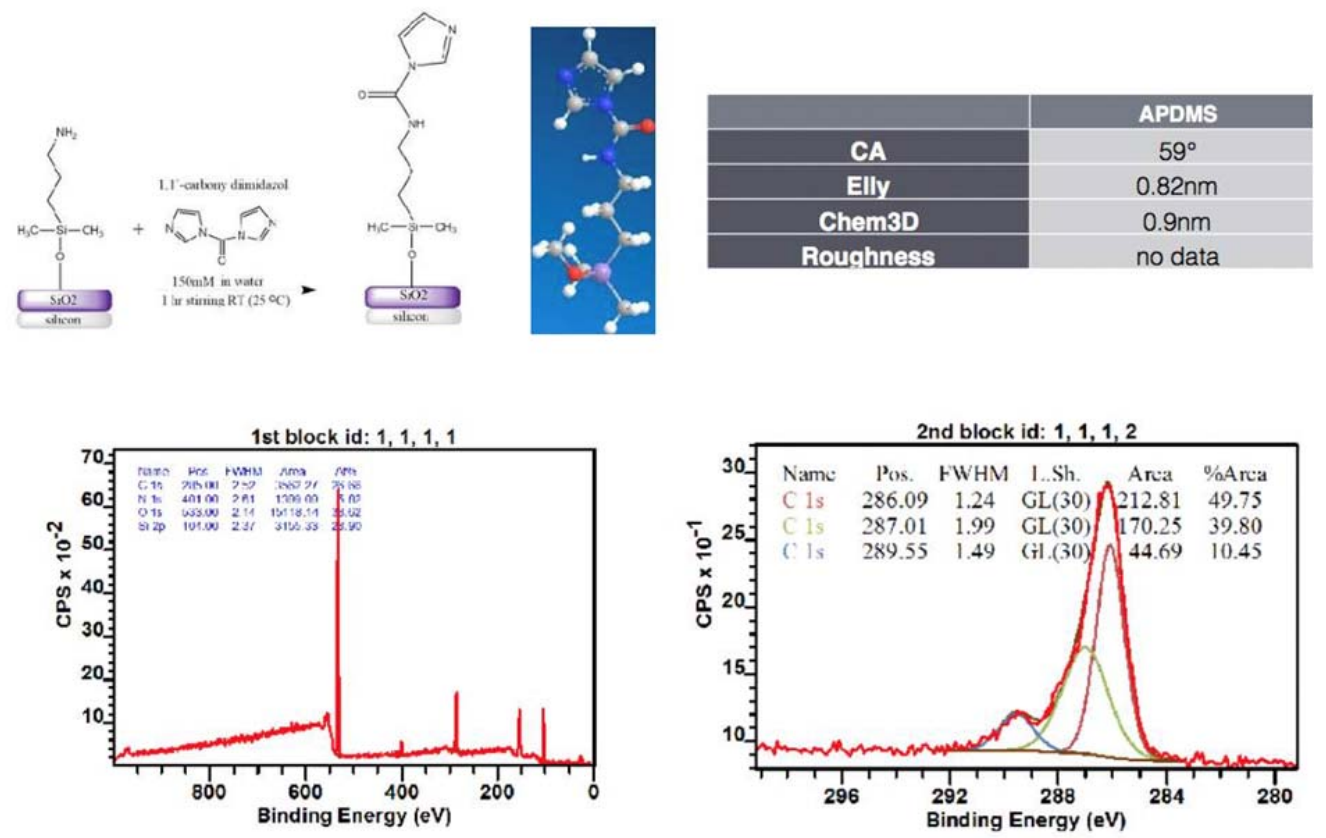

Figure 99: Characyerization analyses from $\mathrm{SiO}_{2}$ samples modified with APDMS and activated with CDI.

Ellypsiometry value increased to $0.82 \mathrm{~nm}$ after CDI activation. This result is very close to the $0.9 \mathrm{~nm}$ expected according the 3D structure calculation. Because after the activation, the surface became more hydrophilic, the contact angle decreased to $59^{\circ}$. The narrow scan of the carbon measured with XPS, clearly show the pick corresponding to the binding energy of the carbonyl group, formed by the reaction of amine group $\mathrm{NH}_{2}$ from APDMS, and the CDI. (Figure 99).

Nevertheless to truly know the effectiveness of the surface modification protocol 
developed until this point, it was essential to test the immobilization of biomolecules in the amine, activated area.

\section{- BSA immobilization}

Firstly, there were incubated $50 \mu \mathrm{g} / \mathrm{mL}$ Bovine Serum Albumin (BSA) over night at $36^{\circ} \mathrm{C}$ in humid conditions. BSA was labeled with Alexia Fluorophore 488 to be analyzed under the fluorescence microscope. It was energetically rinsed with PBS-T and pure water, and dried with $\mathrm{N}_{2}$ gas. The drop of BSA did not cover the whole surface of the $\mathrm{SiO}_{2}$ chip, but only a specific area in order to have a background free of BSA for the fluorescence analysis. The border between the drop and the area with no drop can be noticed in figure 100, were there are presented some pictures taken with the fluorescence microscope.

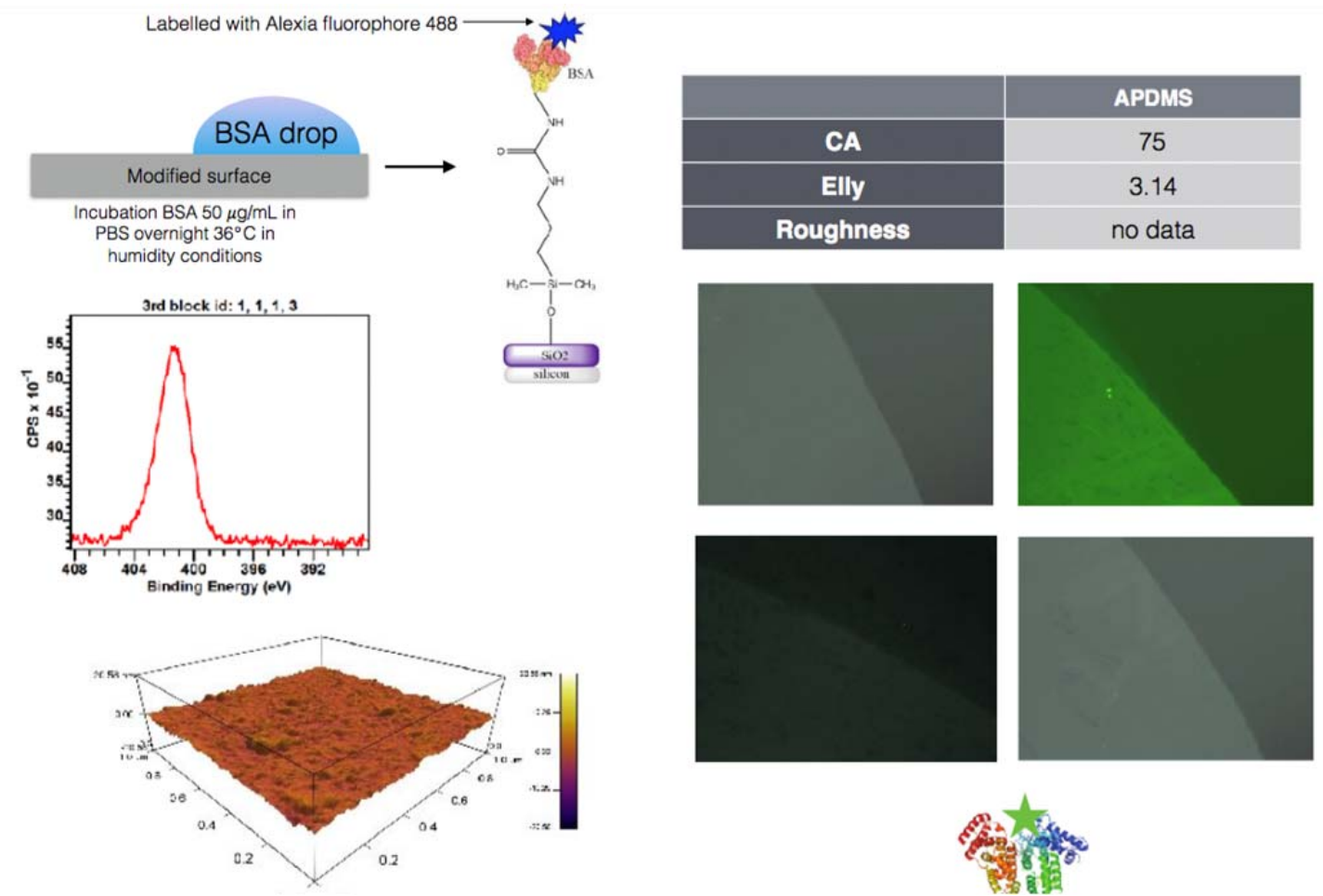

Figure 100: Characterization analyses from $\mathrm{SiO}_{2}$ samples modified with APDMS and activate with CDI and immobilized with BSA.

In ellypsometry it was obtained a thickness of $3.14 \mathrm{~nm}$, which is within the theoretical size of BSA. [Seitz, 2005]. The contact angle increased considerably to $75^{\circ}$, indicating that the surface was not that hydrophilic anymore; probably due to the Alexia Fluorophore. Also, the amount of nitrogen was notably increased 
according XPS results. AFM suggested a good roughness and homogeneity of the characterized surface.

\section{- Antibody immobilization}

Then, the same protocol of surface modification was accomplished for the antibodies immobilization. Two types of antibodies were incubated: anti-S100A6 and anti-MMP9, both of them were shipped from UPM, where the GOFB had already tested them, with other proved immobilization technique, based on a polymeric resist. [Santamaría, 2017]. In order to achieve a better orientation of the antibody in which the binding sites remain free and available for analyte recognition, it was used a protein linker: Protein G. It binds preferably to the area of the constant fraction of the antibodies (base of the "Y"), leaving Fabs available for antigen binding. Its performance has been shown in the scientific literature. [Johnsson, 1991], [Ko, 2009].

The steps of this biofunctionalization protocol are presented in the figure 101: Firstly the linker protein (protein G) was incubated during in one hour and secondly $50 \mu \mathrm{g} / \mathrm{mL}$ of the antibody in PBS was incubated overnight in the whole surface chip. This process was repeated for the different antibodies with and without protein G. The characterization techniques used for antibodies attachment analyses were mainly based on XPS and AFM equipments. 


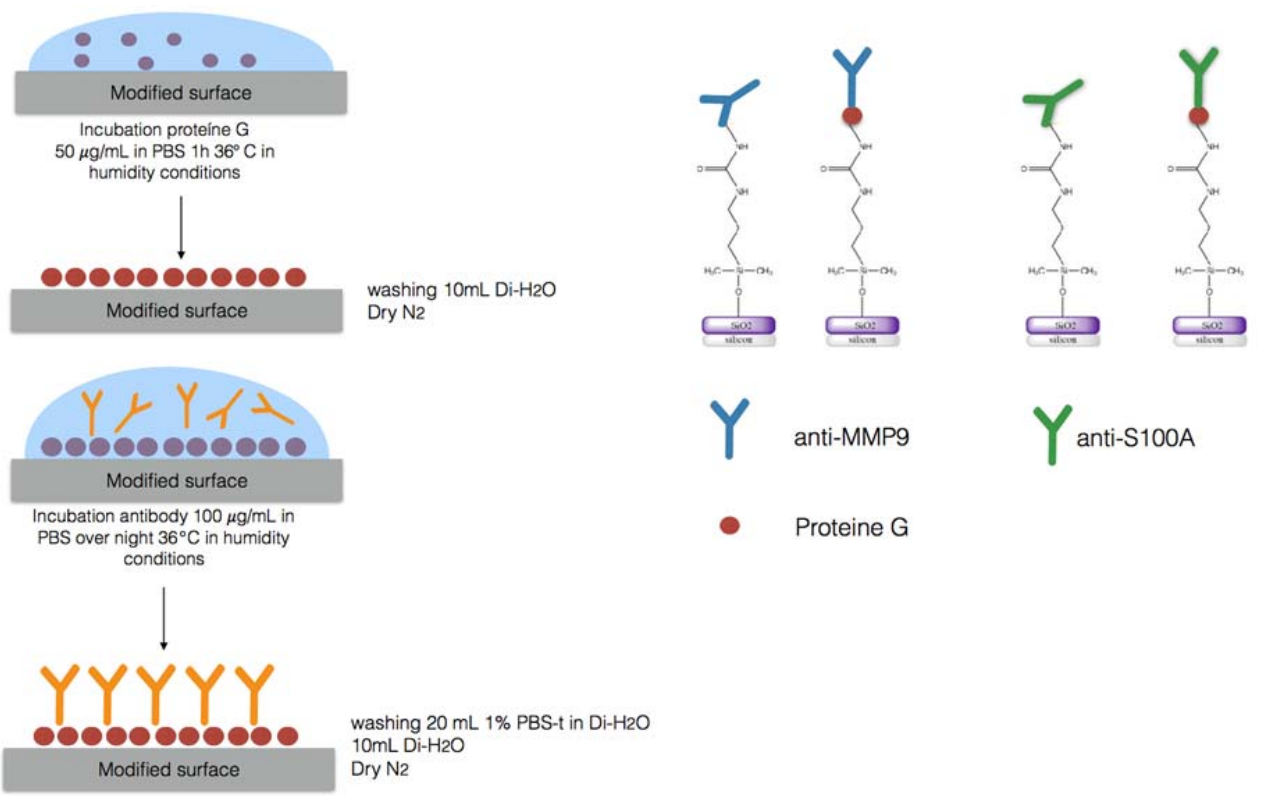

Figure 101: Scheme of the incubation of the two different antobodies and protine G to analyze.

In figure 102 there are presented the results of the anti-MMP9 incubation. According to literatures [McArthur, 2014], XPS results suggest the antibody was successfully attached to the modified surface with APDMS and CDI. Also AFM images showed high surface density and a lower roughness regarding nonbiological surfaces, which indicated the surface had been fully coated with the antibody.
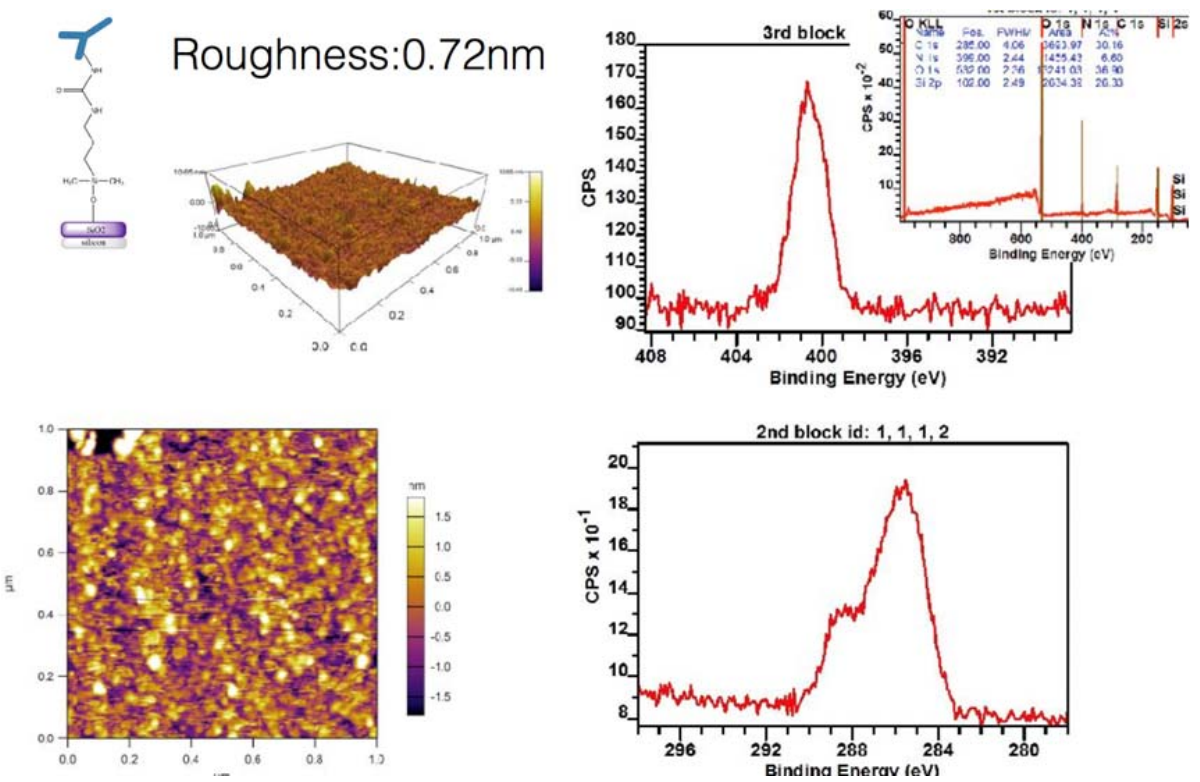

Figure 102: Characterization analyses form $\mathrm{SiO}_{2}$ samples immobilized with anti-MMP9. 
Same process was carried out but with the use of the protein $G$ for a better orientation of the antibody (anti-MMP9). (Figure 103). Roughness was decreased after the use of the protein $G$ and thus, it could be assumed that it has a positive effect on the antibody orientation, enhancing the homogeneity of the surface caused by a constant position of the antibody over the surface. Even though the best way to assess thess preliminary conclusions it would be, by performing the recognition of the specific analyte. More recognition signal implies greater availability of the binding sites and thus, better orientation.
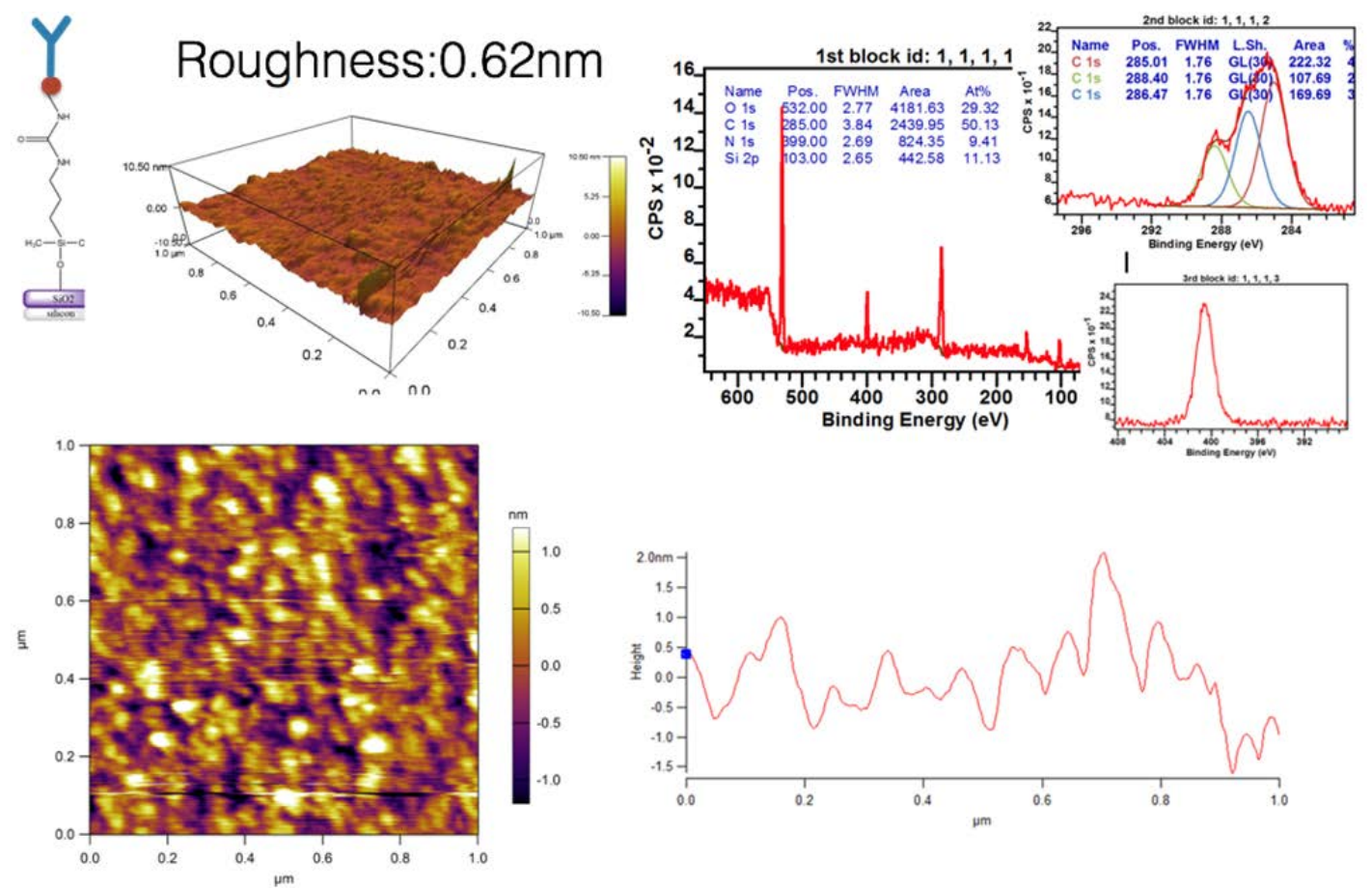

Figure 103: Characterization analyses form $\mathrm{SiO}_{2}$ samples immobilized with anti-MMP9 and proteine $G$.

Same analyses were performed for the incubation of the anti-S100A6 with the protein $\mathrm{G}$ and without protein $\mathrm{G}$. The results also indicated the protein $\mathrm{G}$ has a positive effect on surface homogeneity. (Figure 104 and figure 105). 


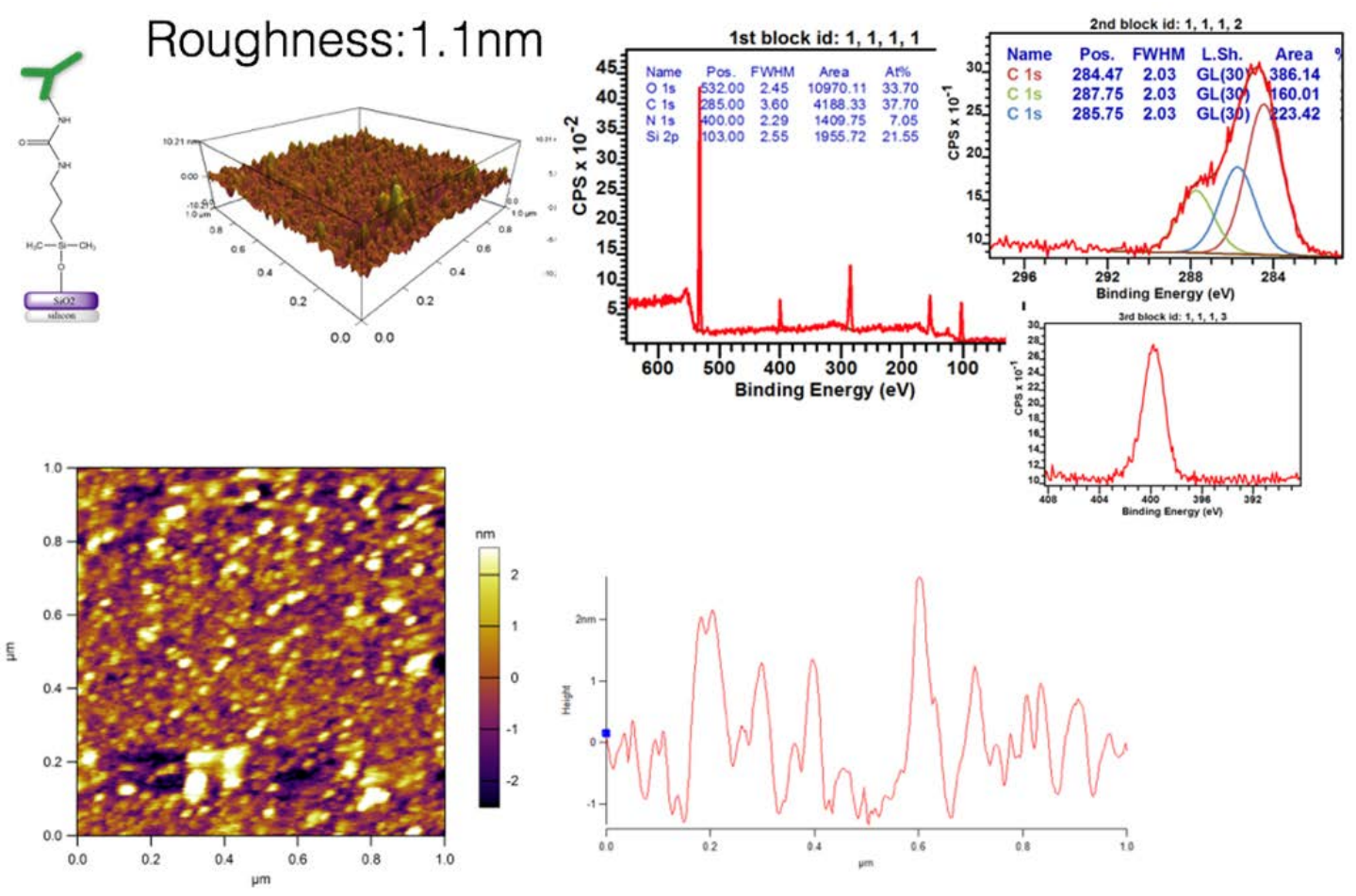

Figure 104: Characterization analyses form $\mathrm{SiO}_{2}$ samples immobilized with anti-S100-A6.

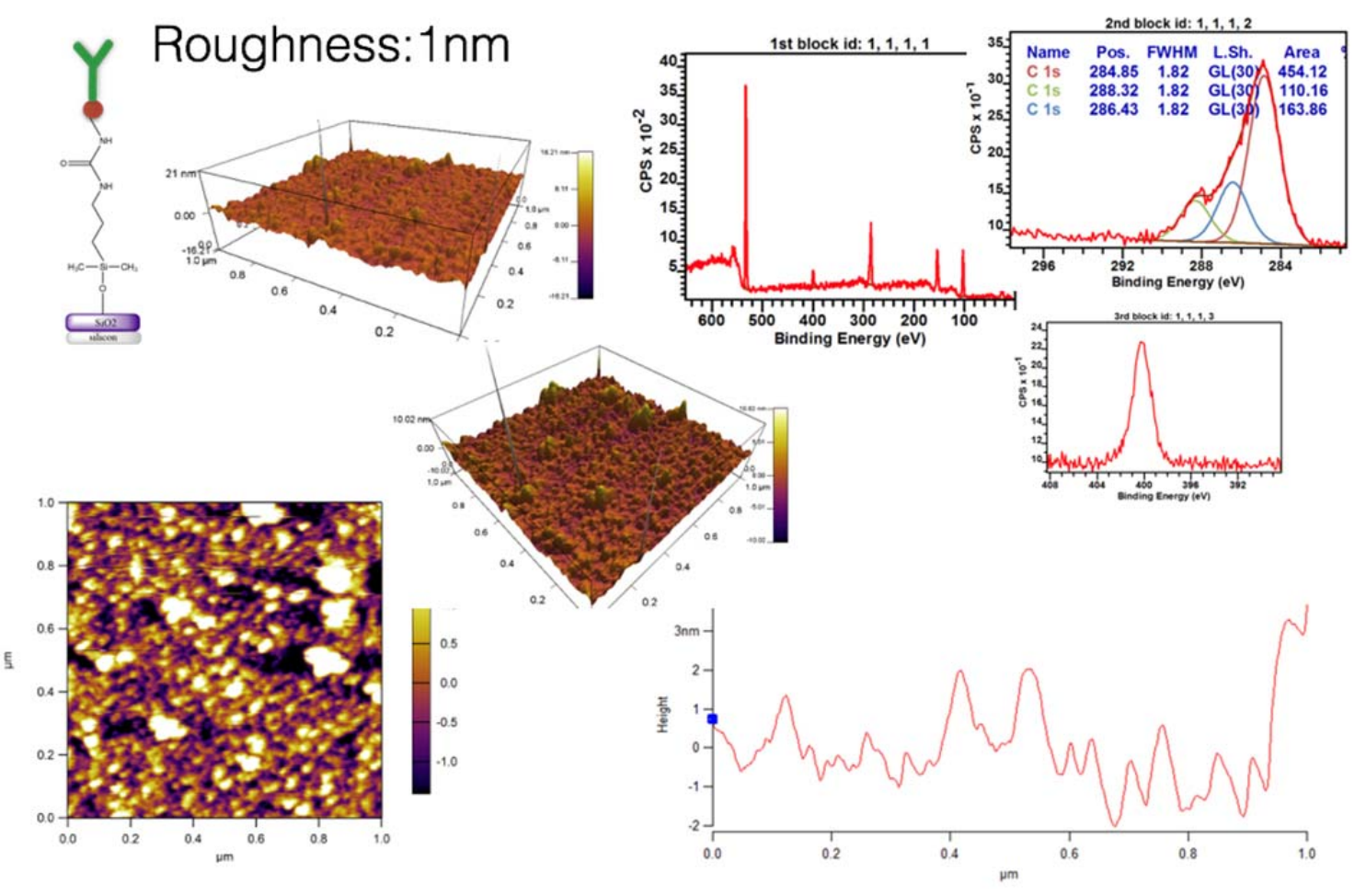

Figure 105: Characterization analyses form $\mathrm{SiO}_{2}$ samples immobilized with anti-S100-A6 and protein $G$. 
All these analytical results, suggest that, the surface modification protocol and the biofunctionalization strategies developed in Wageningen were truly successful and that the process is stable and repetitive. A summary is shows in figure 106.
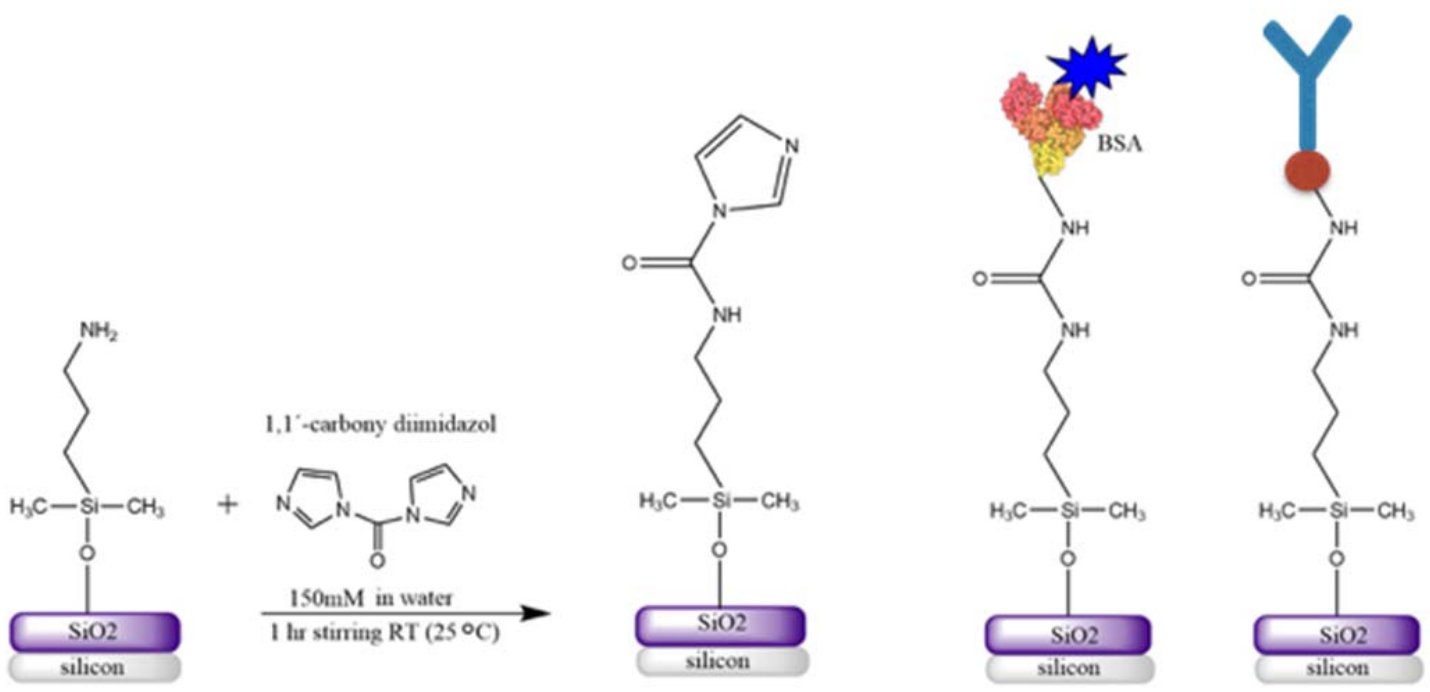

Figure 106: Summary of the surface modification steps and biofunctionalization analyzed.

Next step to conclude this large and complex scientific work is on one side, to perform the characterization of the samples with the reflectometry technique used in the GOPB, and compare the results with the surface analytic techniques used in ORC-WUR. On the other hand, it is expected to develop the exact surface modification and biofunctionalization protocol over the R-NPs.

\subsection{Summary}

In this chapter it was studied the viability to use the R-NPs as label-free optical biosensors. For that reason, there were accomplished several immunoassays experiments over R-NPs demonstrating their capability to detect biomolecules. Different operating methods were compared to optimize the biosensing response. Their LoD could be improved by optimizing the biofunctionalization protocol, and also by changing the materials and dimensions of the Bragg reflectors and the central cavity. Currently, for the small weight molecules detections R-NPs could be labeled with nanoparticles, which multiply per ten the intensity of the signal. 
Nevertheless, if using gold nanoparticles they would not be label-free biosensors anymore. Thus the efforts must be addressed on improving the biofunctionalization strategies as well as the optical properties, in order to maximize R-NPs optical response drawn from biological events. In addition, they have a good susceptibility to be fabricated at industrial level and to be reusable; besides, their high performance in dry conditions makes them potentially ideal optical transducers, to be implemented in commercial, portable and real time detection systems. 


\section{GENERAL CONCLUSIONS AND FUTURE PROSPECTS}

\subsection{General conclusions}

In this thesis it is presented a novel optical transducer based on arrays of resonant nanopillars for biochemical and biological detection.

Several conclusions can be extracted from this work:

- The fabrication of periodic nanopillars was combined with distributed Bragg reflectors multistack, leading to the development of R-NPs. It is demonstrated that the fabrication can be accomplished successfully by using typical procedures used in nanotechnology industry and conserving the optical properties of the designed R-NPs. Besides, the fabrication process developed is reproducible and cost-efficient, which turns R-NPs in firm competitors in the biosensors industry. In fact if was found an improvement of one order of magnitude in the Limit of Detection of R-NPs

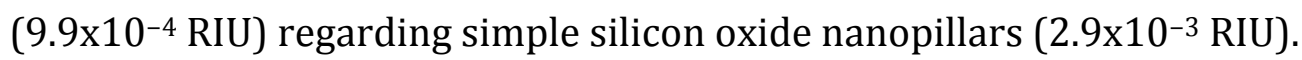

- It has been demonstrated that the $300 \mathrm{~nm}$ pitch R-NPs bulk sensitivity performance (3724.48 cm-1/RIU) doubles the bulk sensitivity of $300 \mathrm{~nm}$ pitch simple SiO2 nanopillars (1652.94 cm-1/RIU). This leads to an increase of the LoD in one order of magnitude for R-NPs (9.9x10-4 RIU) regarding simple SiO2-NPs (2.9x 10-3 RIU) as already pointed in the first paragraph of the general conclusions.

- Besides R-NPs used for biochemical detection revealed improved values of the bulk sensitivity ( $591.54 \mathrm{~nm} / \mathrm{RIU}$ ), obtained in other types of label-free biosensors based on optical reflectometry as for example slot waveguide ring resonators. $182 \mathrm{~nm} / \mathrm{RIU}$ or photonic crystal microcavities $212 \mathrm{~nm} / \mathrm{RIU}$. 
- Also it was found an improved LoD for R-NPs, regarding Localized Surface Plasmon Resonators for chemical detection. (1.8x10-5 and 6.7 $\times 10-5$ RIU respectively).

- R-NPs can be fabricated being distributed along the chip substrate into independent arrays or cells, called BICELLs when they are properly biofunctionalized. This distribution in BICELLs converts them into integrated biosensors with multiplexing properties for multianalyte detection in the same chip. It has been demonstrated the possibility to interrogate 12 single transducers simultaneously.

- An optical read-out system has been designed and developed for the vertical interrogation of the R-NPs arrays, avoiding complex light coupling processes. The read-out system integrates user-friendly software for easy optical response interpretation. R-NPs can be fabricated over a quartz transparent substrate to facilitate the vertical interrogation from the backside, in order to allow R-NPs to be in contact with the surrounding media. The optical read-out system can be coupled to a R-NPs chip holder fabricated ad-hoc and to a fluidic chip holder developed for continuous flow real-time measurements.

- In addition, it can be concluded that R-NPs are suitable to be used as biological sensors. The materials of which they are composed are susceptible to be superficially modified heading to a succesful biofunctionalization process, allowing the specific recognition of the target analyte. It is shown that the immobilization of the antibodies can be accomplished whether in static incubation overnight or with the continuous flow system reducing considerably the immobilization time, but using a much greater amount of simple. The limit of detection obtained for R-NPs was in the order of $5.82 \mathrm{~nm} / \mathrm{mL}$ which equals the values referenced in the literature as for example the sensitivity found for resonant disk based on a slot waveguide for anti-bovine serum albumin (BSA) detection (1.66 ng/mL). 
- When R-NPs are interrogated in dry conditions, the sensitivity is increases one order of magnitude, regarding the same bioapplication measured with R-NPs in humidic conditions $(0.1716$ and 0.014 nm/2g.mL-1 respectively). This implies that R-NPs could be perfectly used in point of care devices in which continuous flow samples make portability and handing very difficult and thus, discret measurement of the samples are desired.

- Surface modification protocols based on organisilanes, developed in the Organic Chemistry Department of Wageningen University improved the biofunctionalization of the $\mathrm{SiO} 2$ interferometers with antibodies, improving thus the biosensing capacity.

- According to results obtained in this thesis, the future work proposed is focused on the implementation of the biofunctionalization protocols, developed in flat SiO2 interferometers, in the R-NPs for the detection of rotavirus. Also it is concluded that other materials could be used for the development of R-NPs, as for example SiO2/Si Bragg reflector pairs which apriory increases the bulk sensitivity, but biofunctionalization protocols and recognition events are still to be tested.

\subsection{Future Prospects}

The results obtained in this thesis have left the door open to continue working in the development and optimization of the R-NPs to be used as commercial biosensors for Point of Care systems.

\subsubsection{New lines of R-NPs biofunctionalization}

After finishing this doctoral thesis it is planned to continue working in the application of the biofunctionalization protocol, developed in Wageningen, to the R-NPs. The protocol will be tested with a real rotavirus application, purchased by UPM specifically for this purpose and tested in flat Fabry-Perot interferometers 
[Holgado, 2014]. (Figure 107). The detection of the rotavirus with the R-NPs has a great scientific interest due to the big presence of rotavirus in the water bodies from some areas of the planet. [Leclerc, 2002], [Conelly, 2012]. Due to its ease to be spread, the early detection by a high sensitive biosensor is a key point for its prevention and more effective treatment.

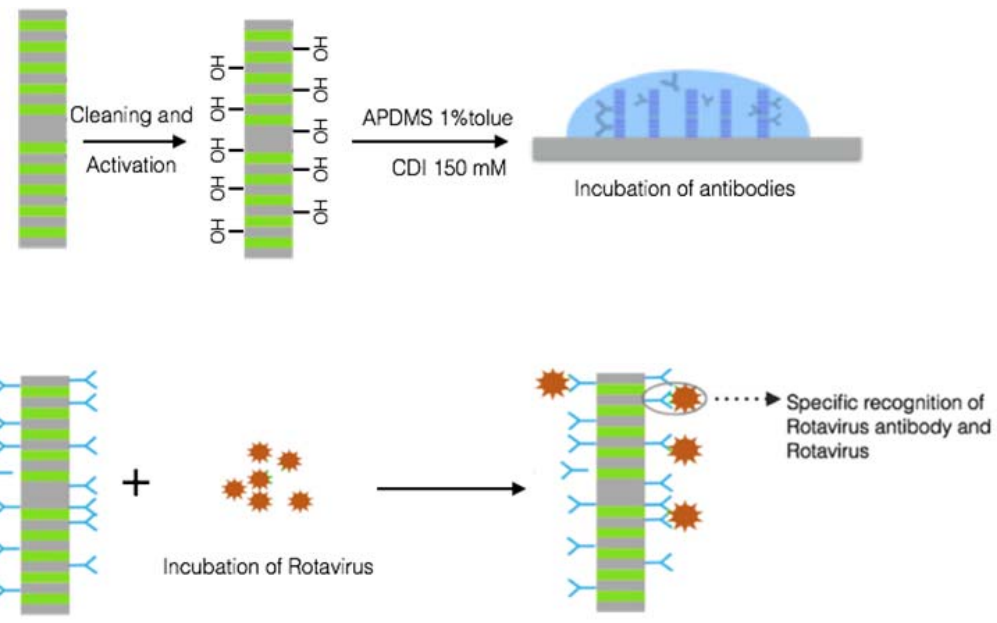

Figure 107: Biofunctionalization of the R-NPs for rotavirus detection.

Also, it is planned to continue working on the research line of surface modification protocols focused on antifouling strategies for specific recognition, in collaboration with the University of Wageningen, in order to optimize biofunctionalization of the R-NPs.

The obtained results will be published as a result of a big effort of both universities in favor of the science and the scientific knowledge exchange.

Once achieved an optimum process for the immobilization of the antibodies and the specific recognition of the analyte, BICELLs could be biofunctionalized with different antibodies for testing samples for multianalyte detection. 


\subsubsection{New materials for R-NPs development}

The improvement of the sensitivity of the R-NPs could be achieved by reducing the diameter of the R-NPs or by increasing the number of Bragg reflectors, but as mentioned in this work, those possibilities are limited by the fabrication parameters. Also, the sensitivity could be improved by increasing the RI contrast of the materials of the Bragg reflectors.

RI contrast between $\mathrm{SiO}_{2}(n=1.4)$ and $\mathrm{Si}_{3} \mathrm{~N}_{4}(n=2)$ is in the order of 0.6 RIU. However, after fabrication of R-NPs, this contrast is reduced to less than 0.15 RIU. An alternative proposed is the use of $\mathrm{SiO}_{2}$ and amorphous silicon $(n=3.9)$. Thus it was designed a new model for R-NPs centering the resonance mode at $850 \mathrm{~nm}$ so as to avoid the absorption from the silicon layers in the visible range. The new design is showed in figure 108.
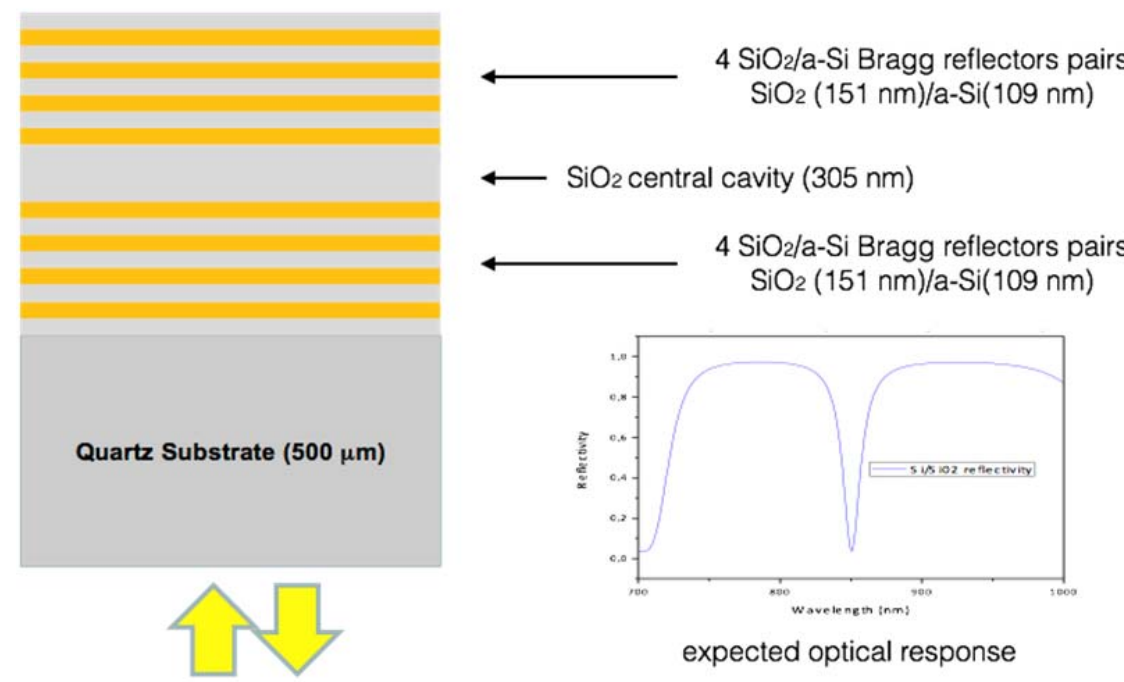

Figure 108: Redesign of the thin film multistack with $\mathrm{Si}_{/} \mathrm{SiO}_{2} \mathrm{Bragg}$ reflectors.

R-NPs were fabricated with those Bragg reflector materials and thickness and with $600 \mathrm{~nm}$ of lattice parameter. First preliminary results were obtained by developing a bulk sensing experiment. (Figure 109). 

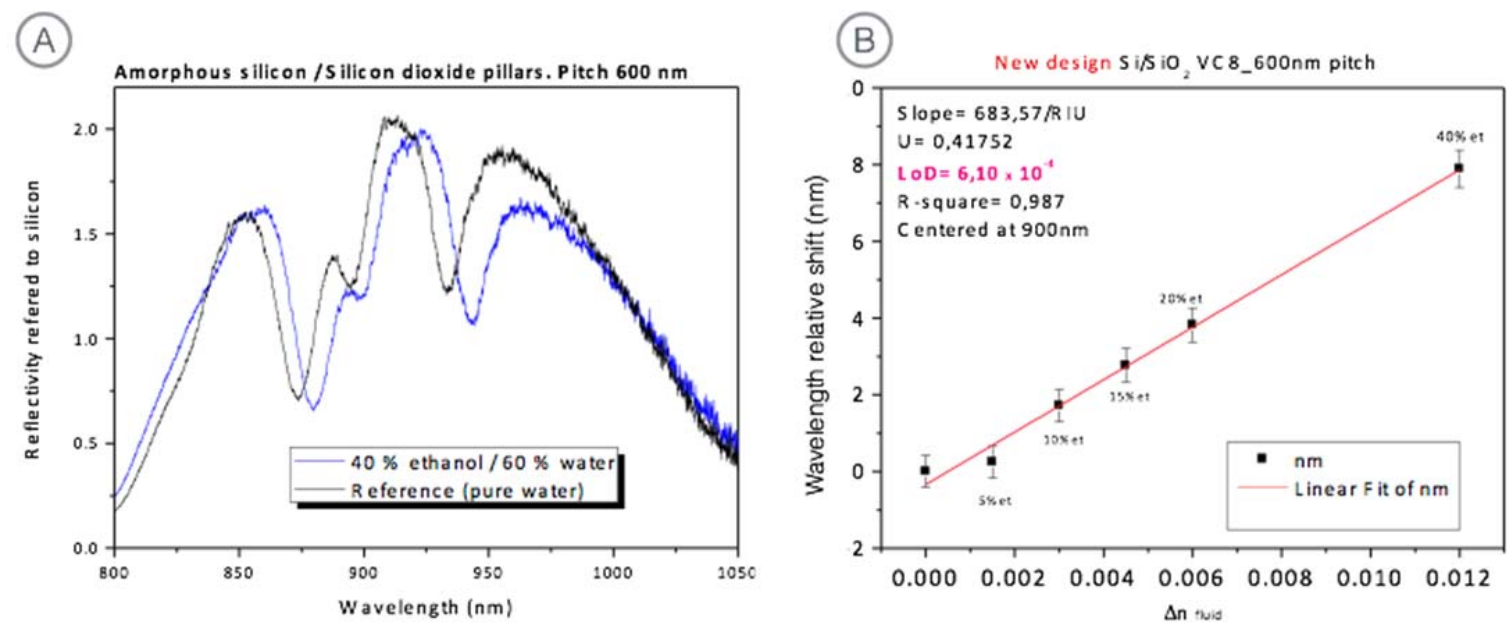

Figure 109: Bulk sensing experiment on R-NPs of Si/SiO2 Bragg reflector. A. Transducing signal. B. Bulk sensitivity.

It was observed that even though the sensitivity increased, regarding $\mathrm{Si}_{3} \mathrm{~N}_{4} / \mathrm{SiO}_{2}$ R-NPs, (683 and $591 \mathrm{~nm} / \mathrm{RIU}$ respectively) the LoD was in the same other. The optical response showed several resonances in the photonic gap suggesting that the effective RI calculated with the Maxwell-Garnett equation for the development of the model did not work properly for this configuration. Thus other method as 3D FDTD must be used for that purpose. If finally a good optical response and bulk sensitivity was achieved with this new proposed materials, it would be necessary to develop an immunoassay, in order to observe the biosensing capacity if the new R-NPs.

Other future work proposed is to measure R-NPs BICELLs, in a portable Point of Care read-out system technology developed by BIOD, company borned from a start-up initiative from Actua UPM. [BIOD]. 


\section{BIBLIOGRAPHY}

\subsection{Publications of the author}

Santamaría, B; Laguna M.F; López-Romero, D; Hernández, A.L; Sanza FJ; Lavín, A; Casquel R; Maigler, M; Espinosa, R.L; Holgado, M; (2017). Development towards compact nitrocellulose-based interferometrc biochps for dry eye MMP9 label-free insitu diagnosis. Sensors. 17(5) 1158.

Hernández, A.L; Casquel, R; Holgado, M; Cornago, I; Fernández, F; Ciaurriz, P; Sanza, FJ; Santamaría, B; Maigler, M; Laguna. MF; (2016). Resonant Nanopillars arrays for label-free Biosensing, Optics Letters , 41(23) 5430-5433.

Fernández, F; Ciaurriz, P; Cornago, I; Casquel, R; Hernández, A; Sanza, FJ; Laguna, MF; Holgado. M; (2016). Optical sensor based on periodic array of resonant nanopillars for real time monitoring, Sensors and Actuators B . 244, 323-326.

Holgado, M; Maigler, M; Santamaría, B; Hernandez, A.L; Lavín, A; Laguna, MF; Sanza, FJ; Granados, D; Casquel, R; Portilla, J; Riesgo, T, (2016). Towards reliable optical label-free Point-of-Care (PoC) biosensing devices, Sensors and Actuators B.236, 765-772.

Cornago, I; Hernández, A.L; Casquel, R; Holgado, M; Laguna, MF; Sanza, FJ; Bravo, J; (2016). Bulk sensing performance comparison between silicon dioxide and resonant high aspect ratio nanopillars arrays fabricated by means of interference lithography. Optical Material Express, 6(7), 2264-2272.

Canalejas-Tejero, V; Hernández, A.L; Casquel, R; Holgado, M; Barrios, C; (2016). Sensitive metal layer-assisted guided-mode resonance SU8 nanopillar array for labelfree optical biosensing. Sensors and Actuators B: Chemical, 226, 204. 
Casquel, R; Soler, J.A; Holgado, M; Hernández, A.L; Lavín, A; de Vicente, J; Sanza, FJ; Laguna, MF; Bañuls, MJ; Puchades, R; (2015). Sub-micrometric reflectometry for localized label-free biosensing, Optics Express. 23(10), 12544-12554.

Laguna, MF; Holgado, M; Hernández, A.L; Santamaría, B; Lavín, A; Soria, J; Suarez, Bardina; Jara, M; Sanza, FJ; Casquel, R; (2015). Antigen-Antibody Affinity for Dry Eye Biomarkers by Label Free Biosensing. Comparison with the ELISA Technique. Sensors and Actuators. 15(8) 19819-29, 2015.

Hernández, A.L; Casquel, R; Holgado, M; Cornago, I; Sanza, FJ; Santamaría, B; Maigler, M; Fernández, F; Lavín, A; Laguna, MF; (2015). Arrays of resonant nanopillars for biochemical sensing, Optics Letters 40(10) 2370-2372.

Laguna, MF; Sanza, FJ; Soria, J; Jara, M; Lavín, A; Casquel, R; Hernández, A.L; Suarez, T; Holgado, M; (2014). Label-free biosensing by means of BICELLs for dry eye, Sensors and Actuators B, 203 209-212.

Laguna, MF; Holgado, M; Sanza, FJ; Lavín, A; Hernández, A.L; Casquel, R; (2014). Optimization of Dengue Immunoassay by Label-Free Interferometric Optical Detection Method, Sensors, 14, 6695-6700.

Holgado, M.; Sanza FJ; Hernández, A.L; Lavín, A; Casquel R; Laguna M.F; (2014). Description of an advantageous optical label-free biosensing interferometric readout method to measure biological species. Special Issue: Photonic Sensors for Industrial, environmental and health monitoring 14, 3675-3689.

\section{Congress}

Santamaría, B; Laguna M.F; López-Romero, D; Hernández, A.L; Sanza FJ; Lavín, A; Casquel R; Maigler, M; Espinosa, R.L; Holgado, M. "BioPhotonics Sensing Cells for in-situ dry eye screening" Biomapp (Santiago de Compostela) 2017. 
Maigler, M; Holgado, M; Santamaría, B; Hernández, AL; Lavín, A; Laguna, MF; Sanza, FJ; Granados, D; Portilla, J; Riesgo, T. “Novel optoelectronic platform for label-free biosensing of influenza detection based on interferometric transducers" Optoel (Santiago de Compostela) 2017.

Casquel, R; Hernández, A.L; Holgado, M; Cornago, I; Fernández, F; Ciaurriz, P; Sanza, FJ; Santamaría, B; Maigler, M; Laguna "Arrays of resonant nano-pillars for refractive index sensing and label free biosensing" Europtrode (Graz) 2016.

López, A; Casquel, R; Holgado, M; Cornago, I; Sanza, FJ; Santamaría, B; Maigler, M; Fernández, F; Lavín, A; Laguna, MF. "Resonant nano-pillars arrays as optical biosensors" Optoel (Salamanca) 2015.

Casquel, R; Soler, J; Holgado, M; López, A; de Vicente, J; Sanza, F.J; Laguna, MF; Bañuls, MJ, Puchades, R. "Sub-micrometric reflectometry for localized label-free biosensing" Optoel (Slamanca) 2015.

Laguna, MF; Holgado, M; Santamaría, B; López, A; Maigler, M; Lavín, A; de Vicente,J; Soria, J; Suarez, T; Bardina, C; Jara, M; Sanza, FJ; Casquel, R; Otón, A; Riesgo, T. "Performance evaluation for different sensing surface of BICELLs biotransducers for dry eye biomarkers". SPIE. 2015.

Sanza, F.J; Laguna, M.F; Casquel, R; López, A; Holgado, M; Lavín, A. “Biosensing comparison between different geometries based on vertical submicron- structrures made of SU-8 resist". EUROMAT 2013.

Sanza, F.J; Rodríguez, D; Laguna, M.F; Casquel, R; Lavín, A; López, A; Holgado, M; Otón. A. "Microfabrication processes for microfluidic devices on a single laser Workstation: direct writing lithography on SU-8, laser ablation on polymers and mask manufacturing” EUROMAT 2013. 
Casquel, R; Lavín, A; Sanza, F.J; Laguna, M.F; López, A; Holgado, M. "Characterization of the performance of optical label-free biosensors" EUROMAT 2013.

Holgado, M; Laguna, MF; Casquel, R; Sanza, FJ; Lavín, A; López, A; Bañuls, MJ; Barrios, CA; Canalejas, V; Puchades, R. "Biophotonic sensing cells (BICELLs) for label-free biosensing". Euromat 2013.

Holgado, M; Sanza, FJ; Laguna, MF; Casquel, R; Lavín, A; López, A; Bañuls, MJ; Barrios, CA; Canalejas, V; Puchades, R. “Biophotonic sensing cells for label-free biosensing". Optoel 2013.

Holgado, M; Sanza, FJ; Laguna, MF; Casquel, R; Lavín, A; López, A; Bañuls, MJ; Barrios, CA; Canalejas, V; Puchades, R. "Biophotonic sensing cells for measuring biological agents". Chicago 2013.

\subsection{References}

Alonso, 2015 Alonso, J. M., Fabre, B., Trilling, A. K., Scheres, L., Franssen, M. C. R., \& Zuilhof, H. (2015). Covalent attachment of 1-alkenes to oxidized platinum surfaces. Langmuir, 31(9), 2714-2721.

Bañuls, 2013 Bañuls, M. J., Puchades, R., \& Maquieira, A. (2013). Chemical surface modifications for the development of silicon-based label-free integrated optical (IO) biosensors: A review. Analytica Chimica Acta, 777, 1-16.

Barrios, 2007 Barrios, C. A., Gylfason, K. B., Sanchez, B., Griol, A., Sohlstrom, H., Holgado, M., et al. (2007). Slot-waveguide biochemical sensor. Optics Letters, 32(21), 3080-3082.

Barrios, 2008 Barrios, C. A., Bañuls, M. J., Gonzaález-Pedro, V., Gylfason, K. B., Sánchez, B., Griol, A., et al. (2008). Label-free optical biosensing with slotwaveguides. Optics Letters., 33(7), 708-710.

Berendsen, Berendsen, C. W. J., Škereň, M., Najdek, D., \& Černý, F. (2009). 2009 Superhydrophobic surface structures in thermoplastic polymers by interference lithography and thermal imprinting. Applied Surface Sciences. 255(23), 9305-9310. 
$B I O D$

Boyd, 2001

Bravoo.org

Canalejas, 2016 Canalejas-Tejero, V., López, A., Casquel, R., Holgado, M., \& Barrios, C. A. (2016). Sensitive metal layer-assisted guided-mode resonance SU8 nanopillar array for label-free optical biosensing. Sensors and Actuators B. 226, 204-210.

Carlborg, 2010 Carlborg, C. F., Gylfason, K. B., Kazmierczak, A., Dortu, F., Banuls Polo, M. J., Maquieira Catala, A., et al. (2010). A packaged optical slot-waveguide ring resonator sensor array for multiplex label-free assays in labs-onchips. Lab Chip, 10(3), 281-290.

Casquel, 2011 Casquel, R., Holgado, M., Sanza, F. J., Laguna, M. F., Barrios, C. A., LópezRomero, D., Ortega, F. J., Bañuls, M. J., Puchades, R. and Maquieira, A. (2011), Optimization of a label-free biosensor vertically characterized based on a periodic lattice of high aspect ratio SU-8 nano-pillars with a simplified 2D theoretical model. Phys. Status Solidi (c), 8: 1087-1092.

Casquel, 2012 Casquel, R. (2012) Biosensors based on vertically interrogated optofluidic sensing cells. Tesis Doctoral UPM.

Cecchet 2005 Cecchet, F., De Meersman, B., Demoustier-Champagne, S., Nysten, B., \& Jonas, A. M. (2006). One step growth of protein antifouling surfaces: Monolayers of poly(ethylene oxide) (PEO) derivatives on oxidized and hydrogen-passivated silicon surfaces. Langimuir: The ACS Journal of Surfaces and Colloids, 22(3), 1173-1181.

Chang, 2007 Chang, Y., Chou, Q. ., Lin, J., Lee, C. (2007). Fabrication of high-aspectratio silicon nanopillar arrays with the conventional reactive ion etching technique. Applied Physics A, 86(2), 193-196.

Chen 1995 Chen, G. Y., Thundat, T., Wachter, E. A., \& Warmack, R. J. (1995). Adsorption-induced surface stress and its effects on resonance frequency of microcantilevers. Journal of Applied Physics, 77(8), 36183622.

Chigrin, 2004 Chigrin, D. N., Lavrinenko, A. V., \& Torres, C. M. S. (2004). Nanopillars photonic crystal waveguides. Optic Express, 12(4), 617-622.

Chou, 1995 Chou, S. Y., Krauss, P. R., \& Renstrom, P. J. (1995). Imprint of sub-25 nm vias and trenches in polymers. Applied Physics Letters, 67(21), 31143116. 
Choudhury, Choudhury, B. D., Casquel, R., Ba \nuls, M. J., Sanza, F. J., Laguna, M. F., 2014 Holgado, M., et al. (2014). Silicon nanopillar arrays with SiO2 overlayer for biosensing application. Optical Material Express, 4(7), 1345-1354.

Chow, 2004 Chow, E., Grot, A., Mirkarimi, L. W., Sigalas, M., \& Girolami, G. (2004). Ultracompact biochemical sensor built with two-dimensional photonic crystal microcavity. Optics Letters, 29(10), 1093-1095.

Conelly, 2012 Connelly, T; Baeumner, J. (2012). Biosensors for the detection of waterborne pathogens. Analytical and Bioanalytical Chemistry, 402, 117-127.

Cooper 2002 Cooper, M. A. (2002). Optical biosensors in drug discovery. Nature Reviews Drug Discovery, 1(7), 515-528.

Cooper, 2009 Cooper, M.A (2009). Label-free Biosensors: Techniques and Applications. Cambridge University Press.

Cornago, 2016 Cornago, I., Hernández, A. L., Casquel, R., Holgado, M., Laguna, M. F., Sanza, F. J., et al. (2016). Bulk sensing performance comparison between silicon dioxide and resonant high aspect ratio nanopillars arrays fabricated by means of interference lithography. Optical Material Express, 6(7), 2264-2272.

Cross, 2003 Cross, G. H., Reeves, A. A., Brand, S., Popplewell, J. F., Peel, L. L., Swann, M. J., et al. (2003). A new quantitative optical biosensor for protein characterisation. Biosensors and Bioelectronics. 19(4), 383-390.

\section{CSC. Scientific $\underline{\text { http://www.cscscientific.com }}$}

Company

Damborský, Damborský, P., Švitel, J., \& Katrlík, J. (2016). Optical biosensors. Essays 2016 in biochemistry, 60(1), 91-100.

De Juan, 2013 de Juan, E. (2013) Estrategias de Inmobilización de anticuerpos para la detección directa de hormonas mediante Inmunosensores de Resonancia de Plasmón Superficial. Tesis Doctoral. UAB

De Vicente, J. de Vicente, M. Holgado, A. Lavín, F. J. Sanza, R. Casquel, M. F. Laguna. 2014 (2014). Uncertainty in optical bio-sensors due to the spectral displacement of the interferences modes of the transducing signal, OPA. 47, 27-34.

Denninson, Dennison, M. J., \& Turner, A. P. (1995). Biosensors for environmental 1995

monitoring. Biotechnology Advances, 13(1), 1-12. 
Desmaële, 2015 Desmaële, D. Soft-Lithography: SU-8 Coating. , 2015. Available from:<http://www.elveflow.com/soft-lithography-reviews-andtutorials/how-to-get-the-best- process/soft-lithography-su-8-coating>.

Dominguez, Domínguez, S., Cornago, I., García, O., Ezquer, M., Rodríguez, M. J., 2013 Lagunas, A. R., et al. (2013). Design, optimization and fabrication of 2D photonic crystals for solar cells. Photonics and Nanostructures Fundamentals and Applications, 11(1), 29.

Dorvel, 2010 Dorvel, B., Reddy, B., Block, I., Mathias, P., Clare, S. E., Cunningham, B., et al. (2010). Vapor-phase deposition of monofunctional alkoxysilanes for sub-nanometer-level biointerfacing on silicon oxide surfaces. Advanced Functional Materials, 20(1), 87-95.

DxDiscovery $\quad$ http://www.dxdiscovery.com/

Enviguard.net http://www.enviguard.net/

Escorihuela, Escorihuela, J; Pujari, S; Zuilhof, H. (2017). Organic Monolayers by 2017 B(C6F5)3-Catalyzed Siloxanation of Oxidized Silicon Surfaces. Langmuir, 33 (9), 2185-2193

Estevez, 2012 Estevez, M. C., Alvarez, M., \& Lechuga, L. M. (2012). Integrated optical devices for lab-on-a-chip biosensing applications. Laser \& Photonics Reviews, 6(4), 463-487.

Fan, 2008 Fan, X., White, I. M., Shopova, S. I., Zhu, H., Suter, J. D., \& Sun, Y. (2008). Sensitive optical biosensors for unlabeled targets: A review. Analytica Chimica Acta, 620(1-2), 8-26.

Feldman, 1986 Feldman, L; Mayer, J.(1986). Fundamental of surface and thin film analysis. 1st Edition. North-Holland

Fernandez Fernández, F., García-López, Ó., Tellechea, E., Asensio, A. C., \& Cornago, I. 2016 (2016). LSPR cuvette for real-time biosensing by using a common spectrophotometer. IEEE Sensors Journal, 16(11), 4158-4165.

Fernandez, Fernández, F., Ciaurriz, P., Cornago, I., Casquel, R., Hernandez, A. L., 2017

Sanza, F. J., et al. (2017). Optical sensor based on periodic array of resonant nanopillars for real time monitoring. Sensors and Actuators B: Chemical, 244, 323-326.

George, 2009 George, S. M. Atomic Layer Deposition: An Overview. Chemical Reviews, 2009, 110(1) 111-131. 
Global Market https://www.gminsights.com/industry-analysis/biosensors-market

Insights

Goddard, 2007 Goddard J. M.; and Hotchkiss, J. H. (2007) Polymer Surface Modification for the Attachment of Bioactive Compounds. Progress in Polymer Science, 32 (7), 698-725.

Gonzalez, 2005 Gónzalez de Buitrago, J.M. (2005) Técnicas y Métodos de Laboratorio Clínico. 2nm ed. Barcelona (españa): Masson.

Gonzalez- González-Guerrero, A. B., Maldonado, J., Dante, S., Grajales, D., \& Guerrero, 2016 Lechuga, L. M. (2017). Direct and label-free detection of the human growth hormone in urine by an ultrasensitive bimodal waveguide biosensor. Journal of Biophotonics, 10(1), 61-67.

GUM

http://www.bipm.org, Evaluation of measurement dataguide to the expression of uncertainty in measurement (GUM).

Gunda, 2014 Gunda, N. S. K., Singh, M., Norman, L., Kaur, K., \& Mitra, S. K. (2014). Optimization and characterization of biomolecule immobilization on silicon substrates using 3-aminopropyl)triethoxysilane (APTES) and glutaraldehyde linker. Applied Surface Sciences. 305, 522-530.

Hellstrom, Hellstrom, S. L. (2007). Basic Models of Spin-Coating.Available from: 2007 http://large.stanford.edu/courses/2007/ph210/hellstrom1/

Henke 2002 Henke, L., Nagy, N., \& Krull, U. J. (2002). An AFM determination of the effects on surface roughness caused by cleaning of fused silica and glass substrates in the process of optical biosensor preparation. Biosensors and Bioelectronics. 17(6), 547-555.

Hernández, Hernandez, A. L., Casquel, R., Holgado, M., Cornago, I., Sanza, F. J., 2015 Santamaria, B., et al. (2015). Arrays of resonant nanopillars for biochemical sensing. Optic Letters., 40(10), 2370-2372.

Hernandez, Hernandez, A. L., Casquel, R., Holgado, M., Cornago, I., Fernandez, F., 2016 Ciaurriz, P., et al. (2016). Resonant nanopillars arrays for label-free biosensing. Optics Letters, 41(23), 5430-5433.

Hilal, 2015 Nidal, H; Daniel, J. (2015). Encyclopedia of Membranes. Springer.

Holgado, 2010 Holgado, M., Barrios, C. A., Ortega, F. J., Sanza, F. J., Casquel, R., Laguna, M. F., et al. (2010). Label-free biosensing by means of periodic lattices of high aspect ratio SU-8 nano-pillars. Biosensors and Bioelectronics, 25(12), 2553. 
Holgado, 2014 Holgado, M., Sanza, F. J., López, A., Lavín, A., Casquel, R., Laguna, M. F. (2014). Description of an advantageous optical label-free biosensing interferometric read-out method to measure biological species. Sensors, 14(2), 3675-3689.

Homola 2003 Homola, J. (2003). Present and future of surface plasmon resonance biosensors. Analytical and Bioanalytical Chemistry, 377(3), 528-539.

Homola, 2006 Homola, J., \& Piliarik, M. (2006). Surface plasmon resonance (SPR) sensors. In J. Homola (Ed.), Surface plasmon resonance based sensors (pp. 45-67). Berlin, Heidelberg: Springer Berlin Heidelberg.

Huang, 2004 Huang, H. C., Lin, C. I., Joseph, A. K., \& Lee, Y. D. (2004). Photolithographically impregnated and molecularly imprinted polymer thin film for biosensor applications. Journal of Chromatography A, 1027(1), 263.

Iqbal, 2010 Iqbal, M. A. Gleeson, B. Spaugh, F. Tybor, W. G. Gunn, M. Hochberg, et al. (2010). Label-free biosensor arrays based on silicon ring resonators and high-speed optical scanning instrumentation. IEEE Journal of Selected Topics in Quantum Electronics, 16(3), 654-661.

IUPAC $\quad$ https://goldbook.iupac.org/

Janeway, 2001 Janeway CA Jr, Travers P, Walport M, et al. (2001) Immunobiology: The Immune System in Health and Disease. 5th edition. New York: Garland Science. The structure of a typical antibody molecule.

Ji 2006 Ji, R., Lee, W., Scholz, R., GÃ $\llbracket$ sele, U., \& Nielsch, K. (2006). Templated fabrication of nanowire and nanoring arrays based on interference lithography and electrochemical deposition. Advanced Materials, 18(19), 2593-2596.

Johnsson, 1991 Johnsson, B., Lofas, S., \& Lindquist, G. (1991). Immobilization of proteins to a carboxymethyldextran-modified gold surface for biospecific interaction analysis in surface plasmon resonance sensors. Analytical Biochemistry, 198(2), 268-277.

Joshi, 2007 Joshi, M., Pinto, R., Rao, V. R., \& Mukherji, S. (2007). Silanization and antibody immobilization on SU-8. Applied Surface Sciences. 253(6), 3127-3132.

Killard, 1995 Killard AJ, Deasy B, O'Kennedy R, Smyth MR. (1995). Antibodies production, functions and applications in biosensors. TrAC;14:257-266. 
Kim, 2009 Kim, J ;Seidler, P; Wan, L;Fill, C. (2009) Formation, structure, and reactivity of amino-terminated organic films on silicon substrates, Journal of Colloid and Interface Science, 329 (1),114-119.

Ko, 2009 Ko, S., Park, T. J., Kim, H. S., Kim, J. H., \& Cho, Y. J. (2009). Directed selfassembly of gold binding polypeptide-protein A fusion proteins for development of gold nanoparticle-based SPR immunosensors. Biosensors \& Bioelectronics, 24(8), 2592-2597.

Kubo, 2011 Kubo, W., \& Fujikawa, S. (2011). Au double nanopillars with nanogap for plasmonic sensor. Nano Letters, 11(1), 8-15.

Kugel, 2014 Kugel, V., \& Ji, H. F. (2014). Nanopillars for sensing. Journal of Nanoscience and Nanotechnology, 14(9), 6469-6477.

Laguna, $2014 a$ Laguna, M. F., Holgado, M., Sanza, F. J., Lavin, A., Lopez, A., \& Casquel, R. (2014). Optimization of dengue immunoassay by label-free interferometric optical detection method. Sensors, 14(4), 6695-6700.

Laguna 2014 b Laguna, M., Holgado, M., Hernandez, A. L., Santamaria, B., Lavin, A., Soria, J., et al. (2015). Antigen-antibody affinity for dry eye biomarkers by label free biosensing. Comparison with the ELISA technique. Sensors (Basel, Switzerland), 15(8), 19819-19829.

Laibinis, 1991 Laibinis, P. E., Bain, C. D., Whitesides, G. M. (1991). Attenuation of photoelectrons in monolayers of n-alkanethiols adsorbed on copper, silver, and gold. Journal of Phisical Chemistry, 95, 7017.

Lasagni, 2010 Lasagni, A. F., \& Menéndez-Ormaza, B. S. (2010). Two- and threedimensional micro- and sub-micrometer periodic structures using twobeam laser interference lithography. Advanced Engineering Materials, 12(1-2), 54-60.

Lavin 2013 Lavín, A., Casquel, R., Sanza, F. J., Laguna, M. F., \& Holgado, M. (2013). Efficient design and optimization of bio-photonic sensing cells (BICELLs) for label free biosensing. Sensors and Actuators B: Chemical, $176,753$.

Lavin, 2016 Lavín, A. (2016). Caracterización y Optimización de Estructuras Biofotónicas y Técnicas Avanzadas de Interrogación para el Desarrollo de Biosensores Ópticos. Tesis Doctoral, UPM.

Lequin, 2005 Lequin, R. M. (2005). Enzyme immunoassay (EIA)/Enzyme-linked immunosorbent assay (ELISA). Clinical Chemistry, 51(12), 2415-2418. 
Lenclerc, 2012 Leclerc, H; Schwartzbrod, L; Dei-Cas, E. (2002). Microbial Agents Associated with Waterborne Diseases. Critical Reviews in Microbiology, 28(4), 371-409.

Leskelä, 2002 Leskelä, M.; and Rital.M. (2002), Atomic Layer Deposition (ALD): From Precursors to Thin Film Structures. Thin Solid Films, 409(1), 138-146.

Li, 2014 Li, Z., Tang, Y., Zhang, L., \& Wu, J. (2014). Label-free study of the function of ion channel protein on a microfluidic optical sensor integrated with artificial cell membrane. Lab Chip, 14(2), 333-341.

Ling, 2006 Ling, X. Y., Reinhoudt, D. N., \& Huskens, J. (2006). Ferrocenylfunctionalized silica nanoparticles:â€\%o preparation, characterization, and molecular recognition at interfaces. Langmuir, 22(21), 8777-8783.

LioniX http://www.lionix-international.com/

Lopez-Romero, López-Romero, D., Barrios, C. A., Holgado, M., Laguna, M. F., \& Casquel, R. 2010 (2010). High aspect-ratio SU-8 resist nano-pillar lattice by e-beam direct writing and its application for liquid trapping. Microelectronic Engineering, 87(4), 663.

Luff, 98 Luff, B.J; Wilkinson. J, Piehler.J, Hollenbach.U, Ingenhoff.J, and Fabricius.N, (1998). Integrated Optical Mach-Zehnder Biosensor, Journal Lightwave Technol. 16, 583

Mannelli, 2003 Mannelli, I., Minunni, M., Tombelli, S., \& Mascini, M. (2003). Quartz crystal microbalance (QCM) affinity biosensor for genetically modified organisms (GMOs) detection. Biosensors \& Bioelectronics, 18(2-3), 129140.

McArthur, McArthur. S.L, Mishra, G; Easton, C.D. (2014). Applications of XPS in 2014 Biology and Biointerface Analysis. Springer.

Mehrotra, 2016 Mehrotra, P. (2015). Biosensors and their applications: A review. Journal of Oral Biology and Craniofacial Research, 6(2), 153-159.

Monosik, 2012 Monosik, R; Miroslav, S; Ernest, S. (2012). Biosensors - classification, characterization and new trends. Acta Chimica Slovaca, 5(1) 109-120.

Myzska, 1999 Myszka, D. G. (1999). Improving biosensor analysis. Journal of Molecular Recognition, JMR, 12(5), 279-284. 
Nanoscribe Nanoscribe. Nanoscribe GmbH. , 2015Available from:http://www.nanoscribe.de/

Ortega, 2006 Instituto de la Real Academia de Framacia. http://www.ranf.com/

Päivänranta, Paivanranta, B., Langner, A., Kirk, E., David, C., \& Ekinci, Y. (2011). Sub2011 $10 \mathrm{~nm}$ patterning using EUV interference lithography. Nanotechnology, 22(37), 375302-4484.

Pease, 2006 Pease, R. F. W. (1981). Electron beam lithography. Contemporary Physics, 22(3), 265-290.

Prieto, 2003 Prieto, F; Sepúlveda,B; Calle,A; Llobera,A et al., (2003). An integrated optical interferometric nanodevice based on silicon technology for biosensor applications. Nanotechnology, 14, (8), 907.

Phys.org $\quad$ https://phys.org

Shankaran, Shankaran, D. R., Gobi, K. V., \& Miura, N. (2007). Recent advancements in 2007 surface plasmon resonance immunosensors for detection of small molecules of biomedical, food and environmental interest. Sensors and Actuators. 121(1), 158-177.

Rich, 2000 Rich, R. L., \& Myszka, D. G. (2000). Advances in surface plasmon resonance biosensor analysis. Current Opinion in Biotechnology, 11(1), 54 .

Robinson, 2013 Robinson S and Nakkeeran R (2013). Photonic Crystal Ring Resonator Based Optical Filters, Advances in Photonic Crystals, Prof. Vittorio Passaro (Ed.), InTech. Available from: https://www.intechopen.com/books/advances-in-photoniccrystals/photonic-crystal-ring-resonator-based-optical-filters

Rsoft $\quad$ http://www.rsoftdesign.com

Santamaría, Santamaria, B., Laguna, M. F., Lopez-Romero, D., Hernandez, A. L., Sanza, 2017 F. J., Lavin, A., et al. (2017). Development towards compact nitrocellulose-based interferometric biochips for dry eye MMP9 labelfree in-situ diagnosis. Sensors .17(5), 1158.

Sanza, 2011 Sanza, F. J., Holgado, M., Ortega, F. J., Casquel, R., LÃ $\tilde{A}^{3}$ pez-Romero, D., Bañuls, M. J., et al. (2011). Bio-photonic sensing cells over transparent 
substrates for anti-gestrinone antibodies biosensing. Biosensors and Bioelectronics, 26(12), 4842.

Sanza, 2015 Sanza, F.J (205). Develpment and Fabrication of Optical Biosensors based on Biophotonic Sensing Cells (BICELLs). Tesis Doctoral.UPM

Savas, 1996 Savas, T. A., Schattenburg, M. L., Carter, J. M., \& Smith, H. I. (1996). Largearea achromatic interferometric lithography for $100 \mathrm{~nm}$ period gratings and grids. Journal of Vacuum Science \& Technology B: Microelectronics and Nanometer Structures Processing, Measurement, and Phenomena, 14(6), 4167-4170.

Scarmozzino, Scarmozzino, R; Gopinath, S; Pregla, R \&Helfer, St. (2000). Numerical 2000 techniques for modeling guided-wave photonic devices. IEEE Journal of Selected Topics in Quantum Electronics, 6(1), 150-162.

Schmidt, 2000 Schmidt, H.; and Menning, M. (2000). Wet Coating Technologies for Glass. Available from:<http://www.solgel.com/articles/Nov00/mennig.htm>.

sea-on- $a$ - $\quad$ http://www.sea-on-a-chip.eu

chip.eu

Sepulveda, Sepulveda, B., Angelomé, P. C., Lechuga, L. M., \& Liz-Marzaín, L. M. 2009

Seitz, 2005 Seitz, R; Brings, R; Geiger, R. (2005).?Protein adsorption on solid-liquid interfaces monitored by laser-ellipsometry. Applied Surface Science, $252,154-157$.

Shang, 2012 Shang,J; Cheng, F, M. Dubey, J.M. Kaplan, et al; (2012), An Organophosphonate Strategy for Functionalizing Silicon Photonic Biosensors. Langmuir 28, 3338-3344.

Snell Law $\quad$ https://www.britannica.com/science/Snells-law

Song, 2006 Mandy LY Sin, Kathleen E Mach, Pak Kin Wong and Joseph C Liao (2014). Advances and challenges in biosensor-based diagnosis of infectious diseases. Expert review of molecular dianostis. 14(2) 225244.

Sweccha 2016]. Sweccha,J. (2016). Nanostructured Imaging Surface Plasmon Resonance Biosensing. Doctoral Thesis. WUR.

Tao, 2008 Tao, S. L., Popat, K. C., Norman, J. J., \& Desai, T. A. (2008). Surface modification of SU-8 for enhanced biofunctionality and nonfouling properties. Langmuir, 24(6), 2631-2636. 
Thevenot, 2001 Thevenot D. R., Tòth K., Durst R. A.,Wilson G. S, (2001) Electrochemical Biosensors: Recommended Definitions and Classification. Pure Appl. Chem. 71, 2333.

Trifonov, 2005 Trifonov, T., Rodríguez, A., Servera, F., Marsal, L. F., Pallars, J., \& Alcubilla, R. (2005). High-aspect-ratio silicon dioxide pillars. Physica Status Solidi (a), 202(8), 1634-1638.

Turner, 1987 Turner PF, Karube I, (1987) Wilson GS, editors. Biosensors: Fundamentals and Applications. Oxford: Oxford University Press; 1987, 597-702.

Turner, 2005 Turner, apf (2005), Biosensors and Bioelectronics, 20, 238.

Vasapollo, Vasapollo, G., Sole, R. D., Mergola, L., Lazzoi, M. R., Scardino, A., Scorrano, 2011 S., et al. (2011). Molecularly imprinted polymers: Present and future prospective. International Journal of Molecular Sciences, 12(9), 59085945.

Velasco, 2016 Velasco, S. (2016). Herramienta de tratamiento de datos procedentes de sensores fotónicos. Bachelor final tesis. UPM

Willets, 2007 Willets, K. A., \& Duyne, R. P. V. (2007). Localized surface plasmon resonance spectroscopy and sensing. Annual Review of Physical Chemistry, 58(1), 267-297.

Yang, 2015 Yuan, D., Dong, Y., Liu, Y., \& Li, T. (2015). Mach-zehnder interferometer biochemical sensor based on silicon-on-insulator rib waveguide with large cross section. Sensors, 15(9), 21500-21517.

Yangqing, 2010 Yangging.C, Liu, Y., Shen, X., Chang, Z., Tang, L., Dong, W., et al. (2015). Ultrasensitive detection of testosterone using microring resonator with molecularly imprinted polymers. Sensors, 15(12), 31558-31565.

Yu, 2005 Yu, F., Li, P., Shen, H., Mathur, S., Lehr, C. M., Bakowsky, U., et al. (2005). Laser interference lithography as a new and efficient technique for micropatterning of biopolymer surface. Biomaterials, 26(15), 23072312.

Zancheta, 2017 Zanchetta,G; Lafranco, R; Giavazzi, F. (2017).Emerging applications of label-free optical biosensors. Nanophotonics, 6(4).

Zetier, 2000 Zetie, K. P., Adams, S. F., \& Tocknell, R. M. (2000). How does a machzehnder interferometer work? Physics Education, 35(1), 46. 
Zhong, 2015 Zhong, YK, Fu,S M, Yan, SL; Chen, PY \& Lin,A; (2015). Arbitrarily-wideband dielectric mirrors and their applications to SiGe solar cells. IEEE Photonics Journal, 7(4), 1-12. 